Supporting Information

\title{
Quantitative Prediction of Hemolytic Toxicity for Small Molecules and Their Potential Hemolytic Fragments by Machine Learning and Recursive Fragmentation Methods
}

Suqing Zheng, ${ }^{a, b^{*}}$ Jun Xiong, ${ }^{a}$ Yibing Wang, ${ }^{c, d}$ Guang Liang, ${ }^{a, b}$ Yong Xu, ${ }^{e}$ Fu Lin ${ }^{a^{*}}$

a: School of Pharmaceutical Sciences, Wenzhou Medical University, Wenzhou 325035, Zhejiang, P. R. China

${ }^{b}$ : Chemical Biology Research Center, Wenzhou Medical University, Wenzhou 325035, Zhejiang, P. R. China

${ }^{c}$ : Genetic Screening Center, National Institute of Biological Sciences, Beijing 102206, P. R. China

d: Tsinghua Institute of Multidisciplinary Biomedical Research, Tsinghua University, Beijing 100084, P. R. China

${ }^{e}$ : Center of Chemical Biology, Guangzhou Institute of Biomedicine and Health, Chinese Academy of Sciences, Guangzhou 510530, Guangdong, P. R. China

*: To whom all correspondence should be addressed. E-mail: lin1449@126.com; zsq_2016@126.com. 


\section{Text S1. Generation of molecular descriptors}

Extended-connectivity Fingerprint (ECFP) (Rogers D, Hahn M (2010) Extended-Connectivity Fingerprints J Chem Inf Model 50:742-754) is extensively used as the molecular descriptor and demonstrates the promising performance in QSAR and ligand-based virtual screening. Thus ECFP is adopted as molecular descriptor in this work. Four ECFPs (1024bit-ECFP4, 2048bit-ECFP4, 1024bit-ECFP6, and 2048bit-ECFP6) are generated for all the curated compounds with our own implementation of ECFP in the e-Hemolytic-Regression program, which could provide the intuitive visualization of each "1" bit of ECFP in the context of 3D structure.

\section{Text S2. Feature selection based on feature importance}

In this work, feature selection is conducted on the basis of the feature importance from the random forest (RF) that will be detailed in the following sections. Thus both full features without feature selection and feature subset after feature selection will be adopted in the model-training.

\section{Text S3. Evaluation metrics to assess the performance of our regression models}

Four evaluation metrics containing the $\mathrm{R}^{2}$, mean absolute error (MAE), mean squared error (MSE), and $\Delta \mathrm{R}^{2}$ (referring to $\mid \mathrm{R}^{2}$ (holdout test set) $-\mathrm{R}^{2}$ (cross-validation) $\mid$ ) are broadly used to monitor the performance of model on the cross-validation dataset, holdout test set and external test set. It should be noted that $\mathrm{R}^{2}$, which is commonly employed to measure the quality of the regression model, is used as the criteria to select the best model.

\section{Text S4. Model training with machine learning methods and full features}

Four machine-learning methods are harnessed to train the regression models, which are concisely described as follows. K-nearest neighbors $(\mathrm{KNN})$ conducts the regression on the basis of the closest instances in the training set. Support Vector Machine (SVM) performs the regression via 
constructing the hyper-planes in multi-dimensional space that separates the distinct classes. Random forest (RF) and Gradient boosting machine (GBM) belong to decision-tree based ensemble methods. RF builds a multitude of decision trees by bootstrap sampling of training-set and randomly selected feature-subset. GBM generates a series of decision trees in a step-wise manner, rather than in a random way as RF. All the key parameters for each method are listed in Table S2, which will be fine-tuned in the cross-validation to achieve the best models with the optimal parameters.

After model-training with the five-fold cross-validation, optimal parameters and the corresponding best models are achieved based on the highest $\mathrm{R}^{2}$. Hence all the combinations of 4 machinelearning methods (KNN, SVM, GBM, and RF), 4 ECFP fingerprints (1024bit-ECFP4, 2048bitECFP4, 1024bit-ECFP6, and 2048bit-ECFP6), full features without the feature selection and 19 random splits of the dataset elicit 304 trained models with the optimal parameters, which are tabulated in Table S2. Subsequently, all those models are evaluated on the respective holdout test sets with the aforementioned four metrics $\left(\mathrm{R}^{2}\right.$, MAE, MSE, and $\left.\Delta \mathrm{R}^{2}\right)$, which are also listed in Table S3.

\section{Text S5. Model training with machine learning methods and feature subsets}

Feature selection is conducted on the basis of the feature importance derived from random forest (RF) method, which was frequently used in the feature selection (Teixeira AL, Leal JP, Falcao AO (2013) Random forests for feature selection in QSPR Models - an application for predicting standard enthalpy of formation of hydrocarbons J Cheminform 5:9-9.). As described in previous section on the model-training without feature selection, 76 runs of RF are performed considering the combination of 4 ECFPs (1024bit-ECFP4, 2048bit-ECFP4, 1024bit-ECFP6, and 2048bitECFP6) and 19 data-splitting schemes, which will result in 76 trained models and 76 sets of feature importance as well. Subsequently, feature importance of all the ECFP bits is sorted in the descending order (Figures S1-S4). Hence, the top 512, 256, and 128 important features (Figures 
S1-S4) are selected respectively as the typical feature subsets, because exhaustive scan of featurenumber, ranging from 1 to the length of ECFP, is computationally expensive for the modeltraining.

Each set of important features combined with four machine learning methods (KNN, SVM, GBM, and RF) is used to train the models respectively. Training procedure is exactly the same as the aforementioned model-training without feature selection. Hence all the combinations of 3 sets of important features (128, 256, and 512 features), 4 machine-learning methods (KNN, SVM, RF, and GBM), 4 ECFPs (1024bit-ECFP4, 2048bit-ECFP4, 1024bit-ECFP6, and 2048bit-ECFP6), and 19 different random data-splitting schemes will produce 912 models according to the highest $\mathrm{R}^{2}$ after the five-fold cross-validation, which are appended to Table S3. Furthermore, all the best 912 models are further assessed on the respective holdout test sets, which are also summarized in Table S3 and plotted in Figure 10. Therefore, by intensive model-training with full feature and feature subsets, 1216 models (M0001-M1216 in Table S3) including 912 models with feature selection and 304 models without feature selection are harvested. In order to reduce the performance bias resulted from different random data-splitting schemes, 64 average models (AM01-AM64) are obtained by averaging over 19 data-partition schemes and their performances are given in Table S4, Table 1 and Figure 10.

\section{Text S6. Y-randomization test of the regression models}

Y-randomization test (Rücker C, Rücker G, Meringer M (2007) Y-Randomization and Its Variants in QSPR/QSAR J Chem Inf Model 47:2345-2357) is performed to check the robustness of all the 1216 models. In this test, the target variable "LogHD $\mathrm{D}_{50}$ " for Dataset-CV are randomly shuffled. (Table S5). Subsequently, five-fold cross-validation on this shuffled dataset is conducted with exactly the same molecular descriptors and protocols as mentioned above. The best models are also determined according to the highest $\mathrm{R}^{2}$ assessed on the internal validation dataset during the crossvalidation, and further evaluated on the hold-out test set (Dataset-Holdout-Test) without any 
random shuffling. The model performances for all the 1216 individual and 64 average regression "models after Y-randomization" are listed in Tables S6-S7 and plotted in Figure S29.

\section{Text S7. Construction of a consensus model}

A consensus model (CM01) is proposed and constructed by selecting 19 best models (Table S8) from Table S3 purely based on the following three criteria: (1) $\mid \mathrm{R}^{2}$ (holdout test set)- $\mathrm{R}^{2}$ (crossvalidation) $\mid \leq 0.05$; (2) the highest $\mathrm{R}^{2}$ (holdout test set); (3) each best model is selected from each data-splitting scheme, since it is not efficient to deploy all 1216 models in practical prediction for users. The performance of this consensus model on the holdout test set is tabulated in Table 2 . Furthermore, CM01 is further evaluated on an external hemolytic toxicity dataset with 70 compounds, which are newly retrieved from the literature published after 2018 June, and affords the performance in Table 3.

\section{Text S8. Definition of the applicability domain of our regression model}

To fully follow the guideline of Organization for Economic Cooperation and Development (OECD), applicability domain of the models should be defined appropriately. It is hypothesized that the compound, which is highly dissimilar from all the compounds used in the model training, may not be predicted with good confidence. With this assumption, the applicability domain of our model is defined on the basis of the concept "average-similarity", which will be introduced as follows.

Each compound in the holdout test set (Dataset-Holdout-Test) is compared with all the compounds in the cross-validation dataset (Dataset-CV) according to the Tanimoto similarity based on 2048bit-ECFP6. Subsequently, five most similar compounds from Dataset-CV are retrieved and treated as the five nearest neighbors for the given compound in Dataset-Holdout- 
Test, and the average of five similarities is defined as "average-similarity" between this given compound and these five nearest neighbors.

According to this definition, each compound in Dataset-Holdout-Test retrieves the five nearest neighbors in Dataset-CV to calculate its "average similarity". Similarly, each compound in Dataset-CV also finds five nearest neighbors in Dataset-CV to compute its corresponding average similarity. Finally, the histograms of average similarity for Dataset-Holdout-Test and Dataset-CV are given in Figure $\mathbf{S 5}$ to address the applicability domain of our models.

\section{Text S9. Implementation of "e-Hemolytic-Regression" program}

"e-Hemolytic-regression" software (Figure 1) is developed based on our previous "e-Bitter" program for the prediction of bitter compound (Zheng S, Jiang M, Zhao C, Zhu R, Hu Z, Xu Y, Lin F (2018) e-Bitter: Bitterant Prediction by the Consensus Voting From the Machine-Learning Methods Front Chem 6). This program has some basic functions such as reading and writing of Tripos Mol2 file and visualization of molecule in 3D viewer. In this program, there are two major components. One is generation and visualization of ECFP based molecular descriptors, which is natively implemented in this program; the other is the quantitative prediction of hemolytic toxicity of small molecules with diverse machine learning methods by evoking Scikit-learn python library (v0.18.2) in Winpython (v3.5.4.0). To fuse both parts, various python scripts have been developed. Besides the main function for the automatic prediction of hemolytic toxicity of small molecules, three extra features are also provided as follows: (1) automatic inspection of applicability-domain of model for the given molecule (Figure 1); (2) intuitive model interpretation for the given compound based on the feature importance (Figures 13, 15, and S35-S41) from random forest and X-scrambling methods. The details of these functions will be further addressed as follows. 
In order to examine whether the given compound is within the applicability domain (AD) of our model, we have implemented a useful function for automatic inspection. Specifically, the applicability domain of our model is defined based on the "average similarity" of given input compound, which is introduced in the previous section (Text S8). According to the blue dot-line in the histogram of average similarity in Figure S5, an average-similarity with the value of $\mathbf{0 . 1 5}$ can be assumed as the threshold to define the applicability domain of our model. Concretely speaking, if the average similarity of the given compound is higher than this threshold, indicating that this compound is located inside of the applicability domain of our model and thereby the prediction for this compound can be regarded as a confident inference, otherwise, the prediction is probably a bold extrapolation.

To well interpret our model, feature importance (FI), which emphasizes the importance of each ECFP fingerprint bit " 1 " contributing to our regression model, is employed. For the sake of intuitive visualization, we have developed a unique function in our program to synchronously view the structural feature in the context of 3D structure and the corresponding feature importance for each ECFP fingerprint bit " 1 ". Technically, the average feature importance, which is derived from RF with the full features of 2048bit-ECFP6, is implicitly integrated into our program for the interactive visualization of ECFP fingerprint-bit "1", its associated 3D structural feature and feature importance. For the demonstration purpose, the structural features and ECFP bits with the top three largest feature importance (FI) are highlighted in Figures 13 and S35-S36. However, this feature importance does not take into account other methods such as SVM, which is used in our ensemble model (e.g., AM14). Due to this limitation, X-scrambling is also applied to derive the important features for our best average model (AM14). An ECFP bit is important, if model performance is decreased after shuffling the bit value, and an ECFP bit is not important if model performance is not apparently changed after shuffling the bit value. Similarly, six most important features are shown in Figure 15, and S37-S41, and discussed in the main text. 


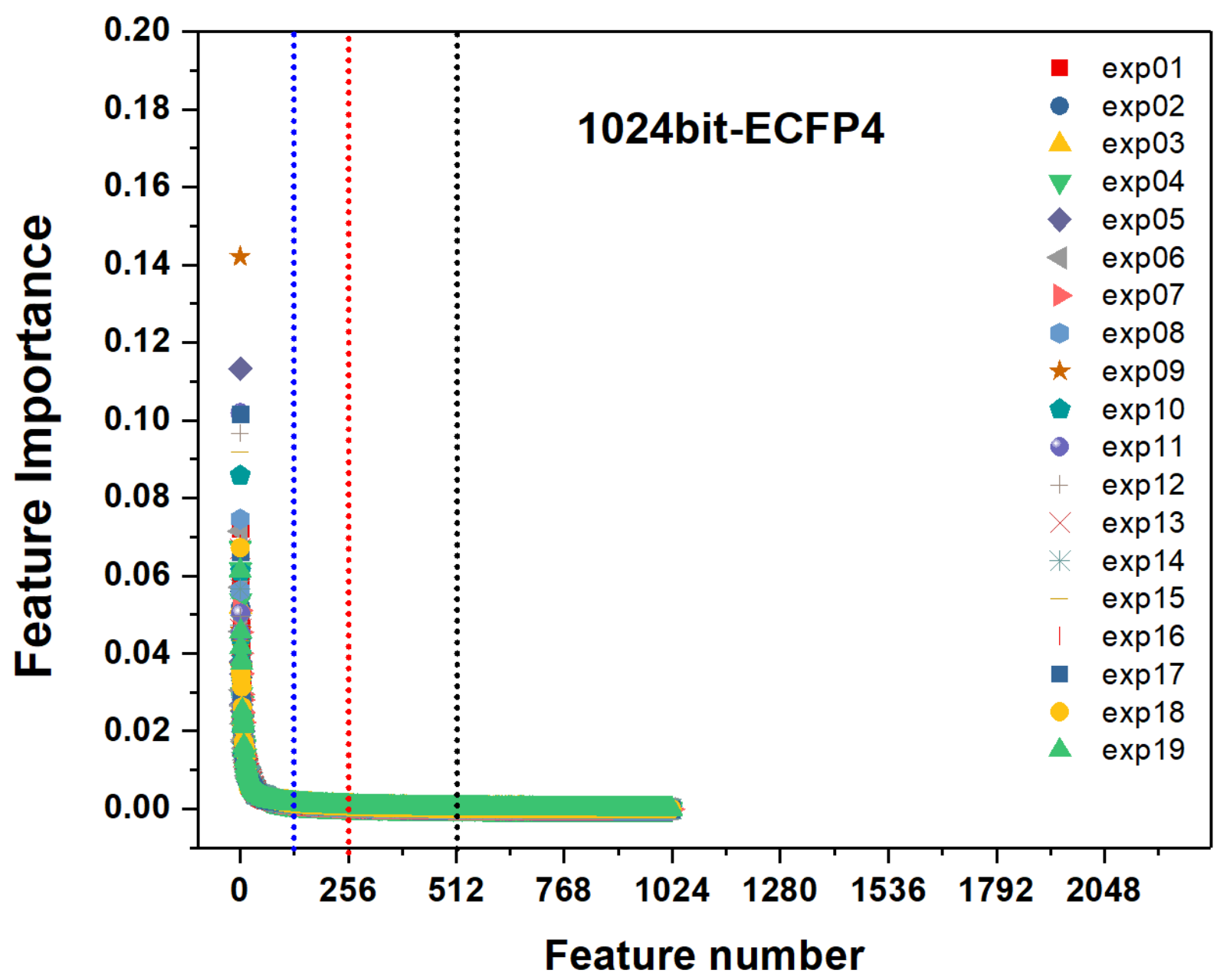

Figure S1. 19 sets of feature importance derived from random forest method with 1024bit-ECFP4. 


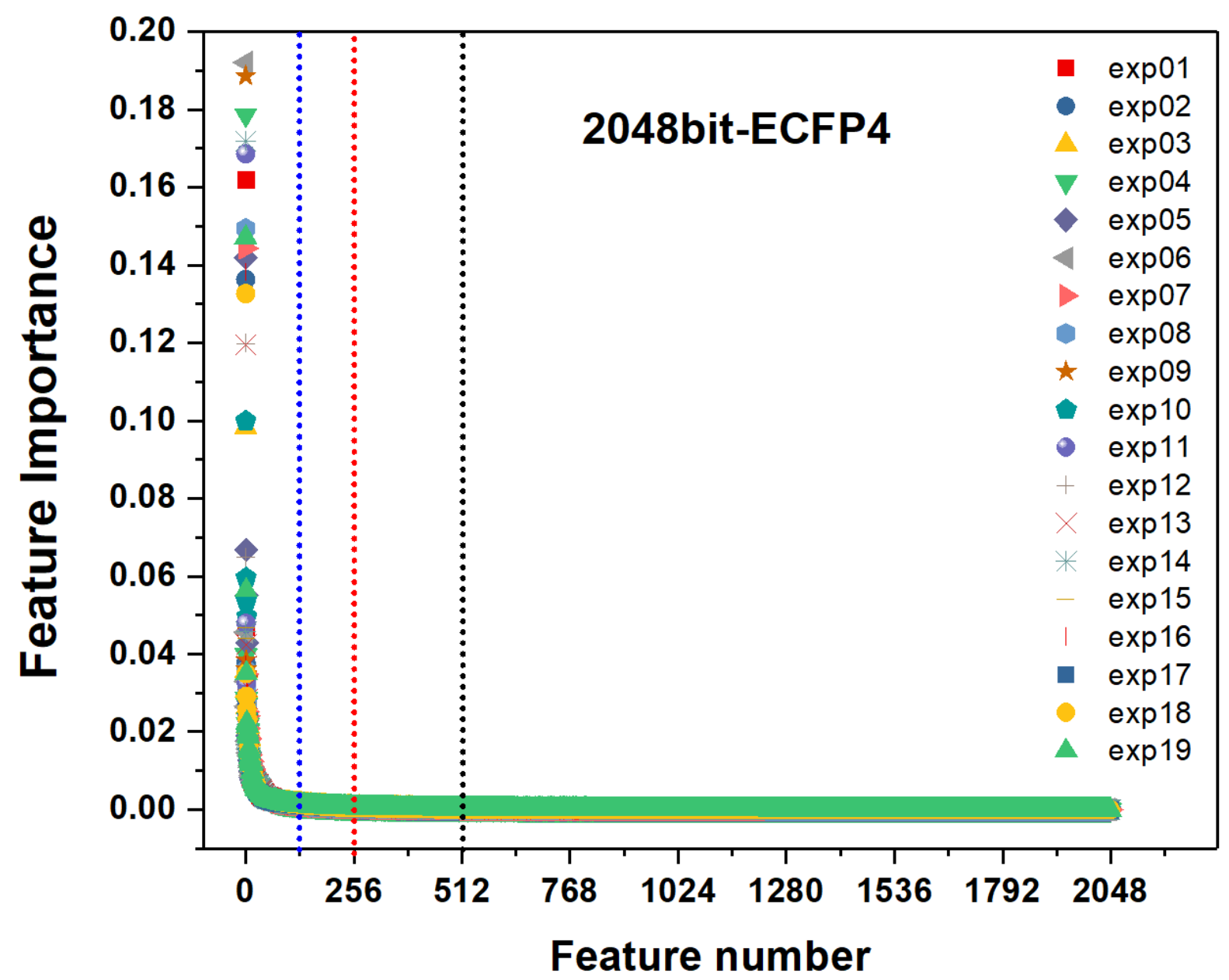

Figure S2 19 sets of feature importance derived from random forest method with 2048bit-ECFP4. 


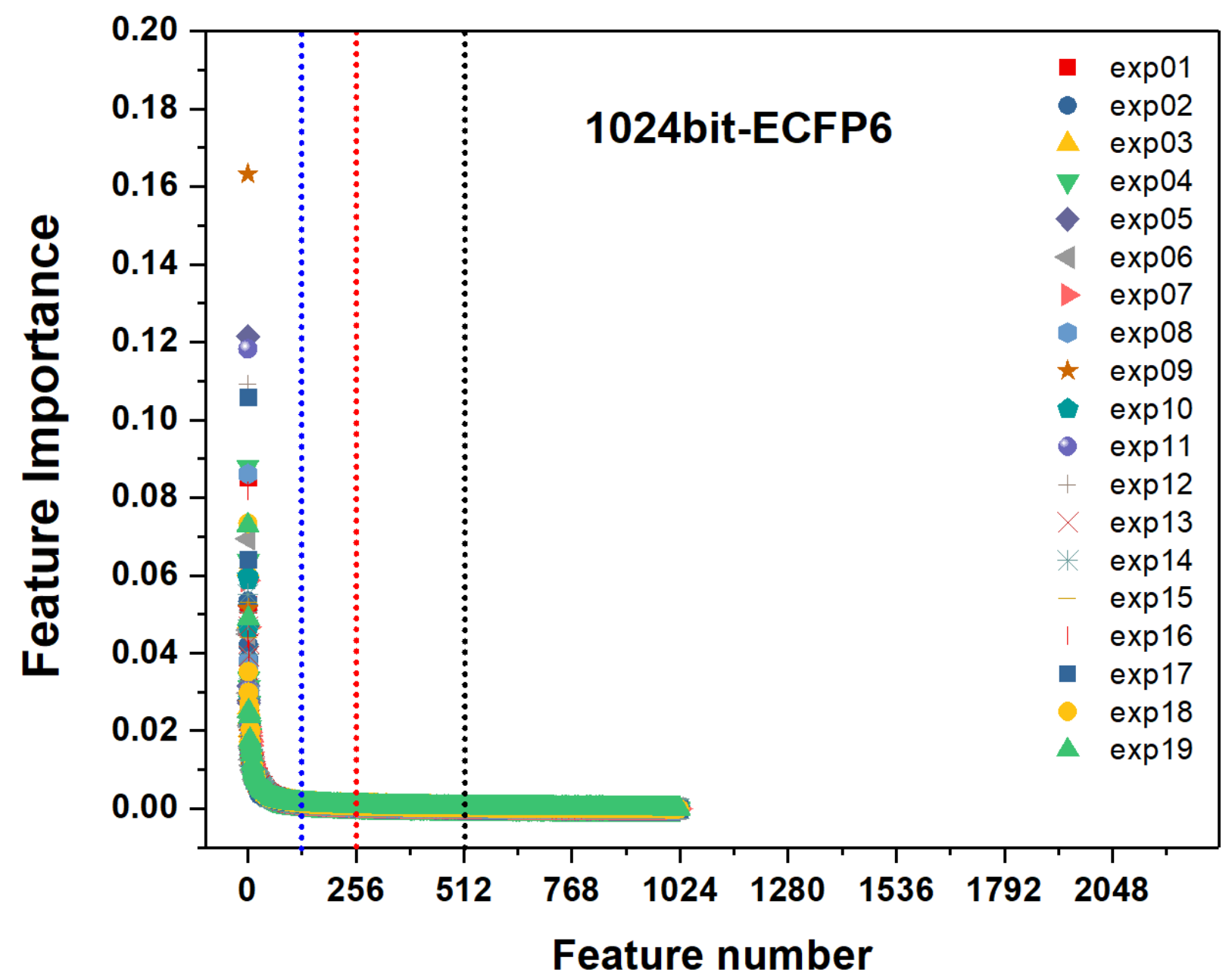

Figure S3. 19 sets of feature importance derived from random forest method with 1024bit-ECFP6. 


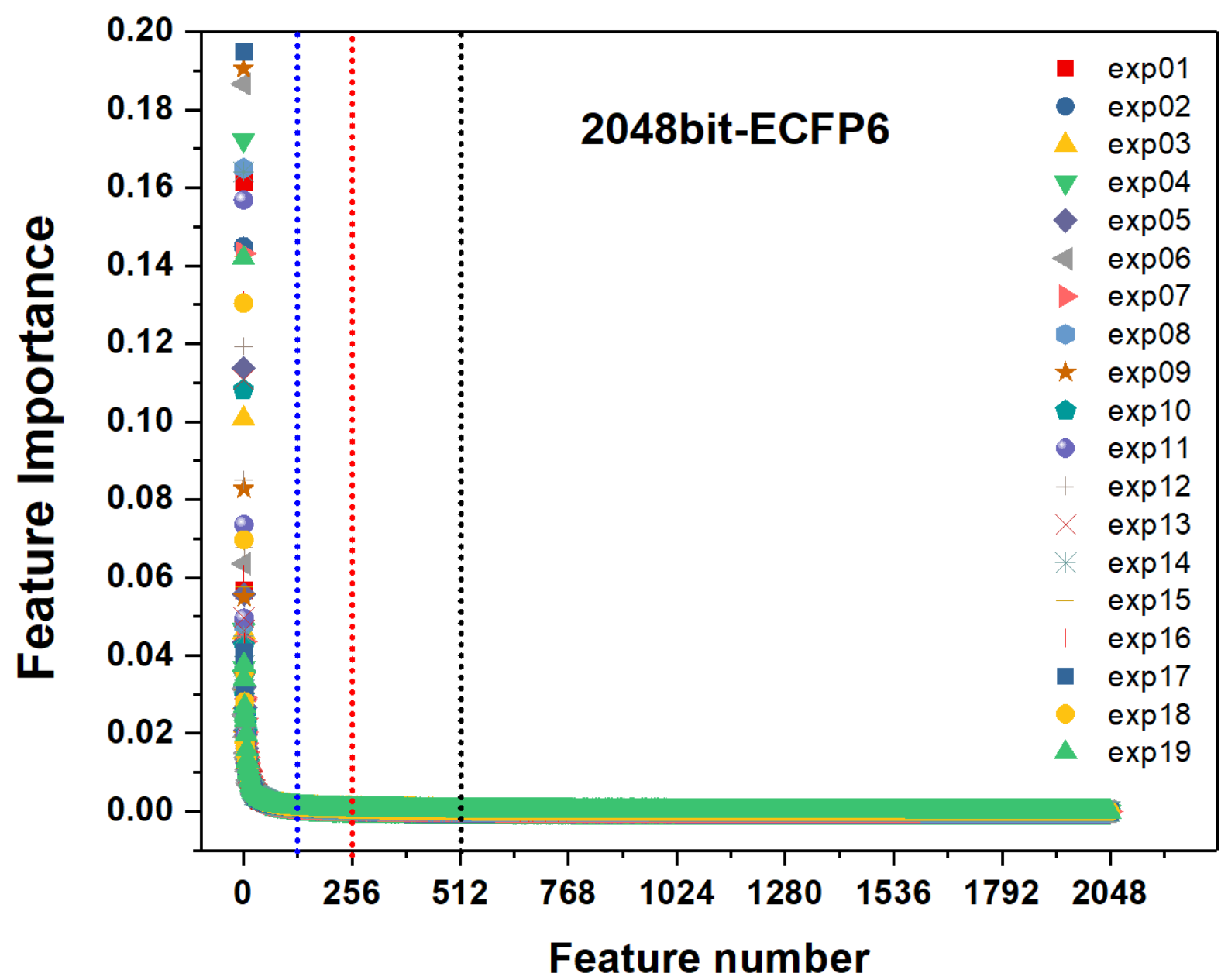

Figure S4. 19 sets of feature importance derived from random forest method with 2048bit-ECFP6. 


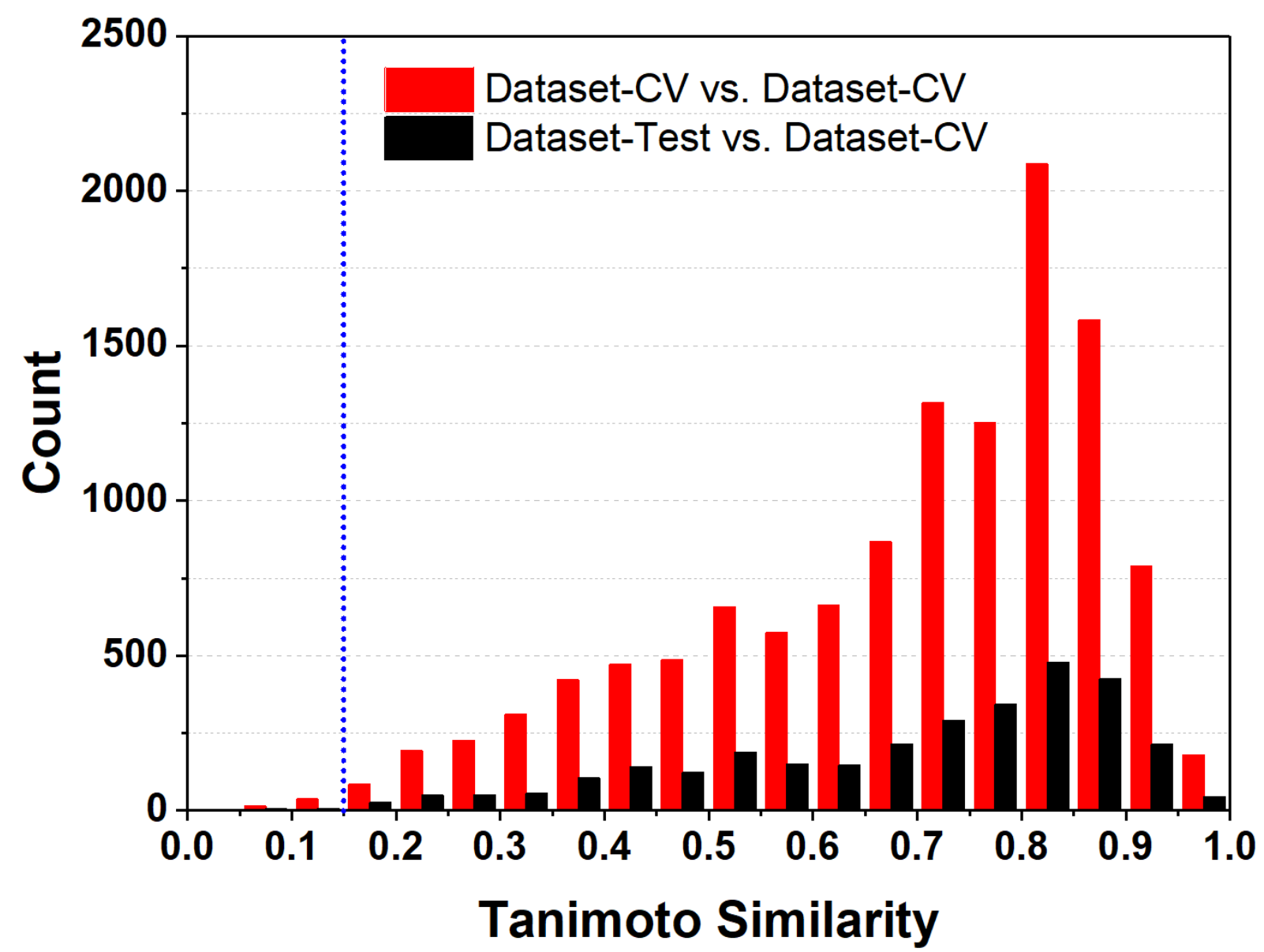

Figure S5. Histograms of average-similarity; The average-similarity threshold of $\mathbf{0 . 1 5}$ (blue dot-line) is used to define the applicability domain of our regression model. The detailed definition of average-similarity is given in Text S8. 


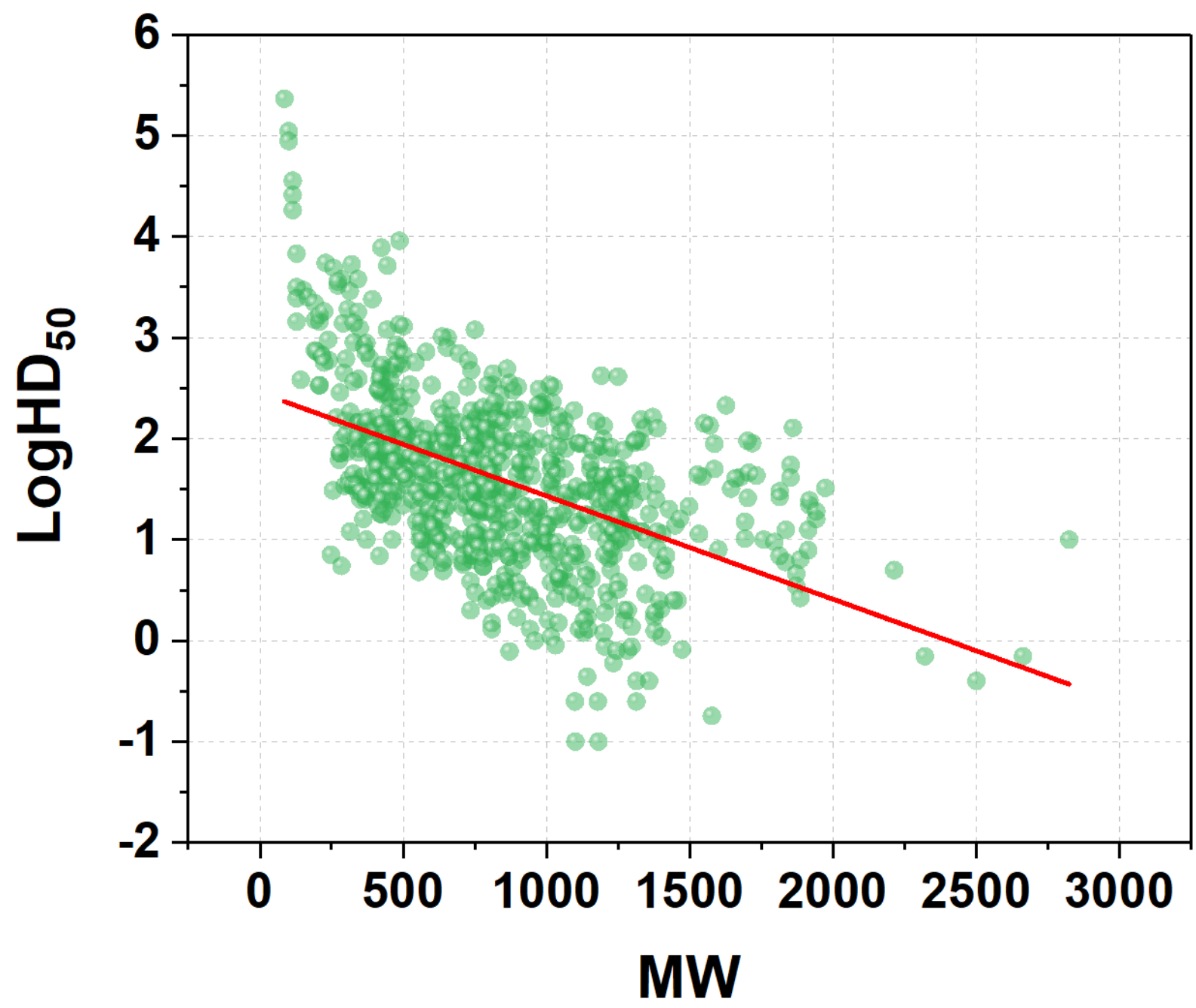

Figure S6. Scatter plot of $\operatorname{LogHD}_{50}$ vs MW for our hemolytic toxicity dataset (805 compounds). The red line is obtained by the linear fit over all the green spheres with a $\mathrm{R}^{2}$ value of 0.26 , suggesting that $\operatorname{LogHD}_{50}$ is weakly associated with MW. 


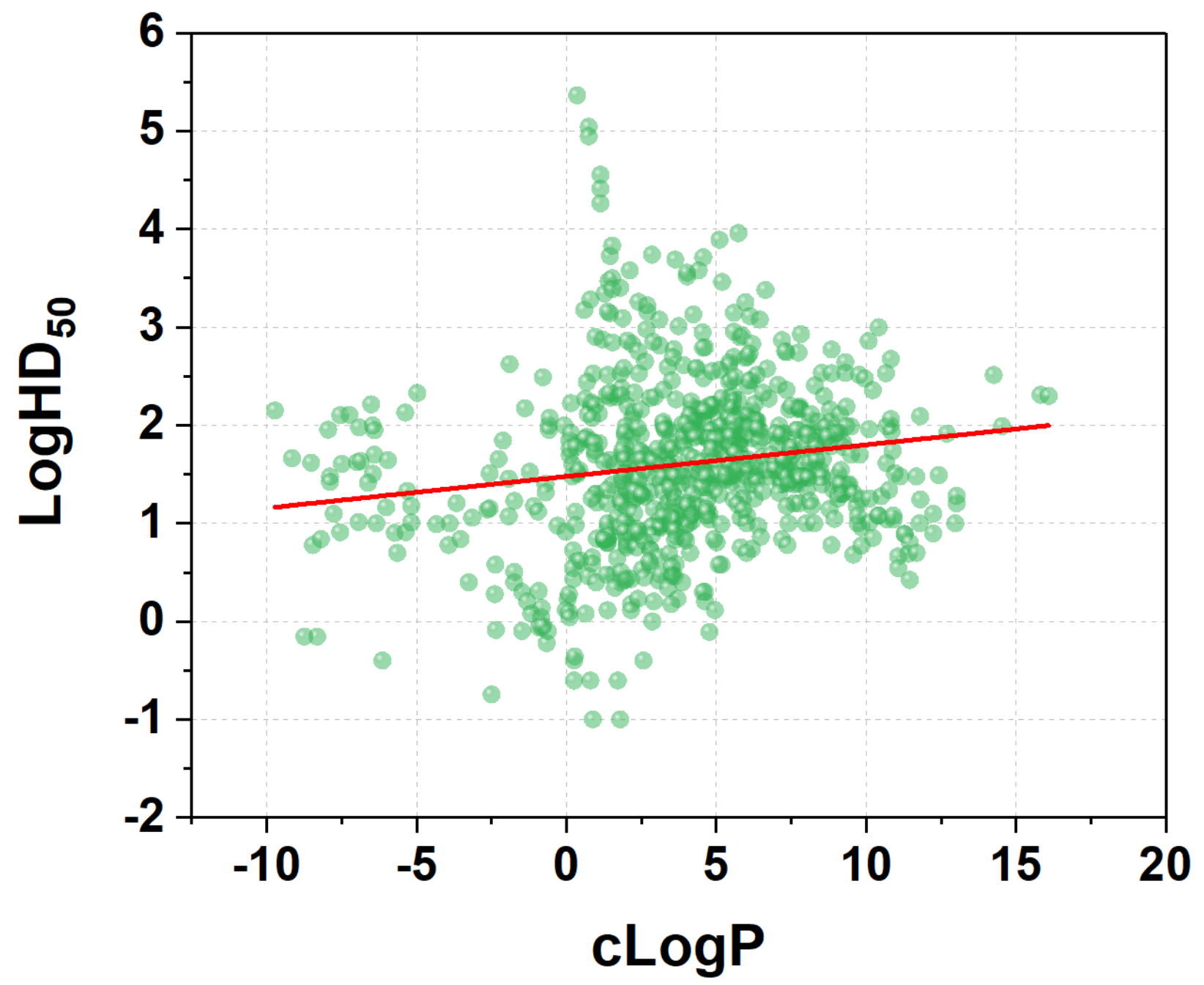

Figure S7. Scatter plot of $\log \mathrm{HD}_{50}$ vs cLogP for the hemolytic toxicity dataset including 805 compounds. The red line is derived by the linear fit over all the green spheres with a $\mathrm{R}^{2}$ value of 0.02 , which indicates that there is almost no correlation between $\operatorname{LogHD}_{50}$ and cLogP. 


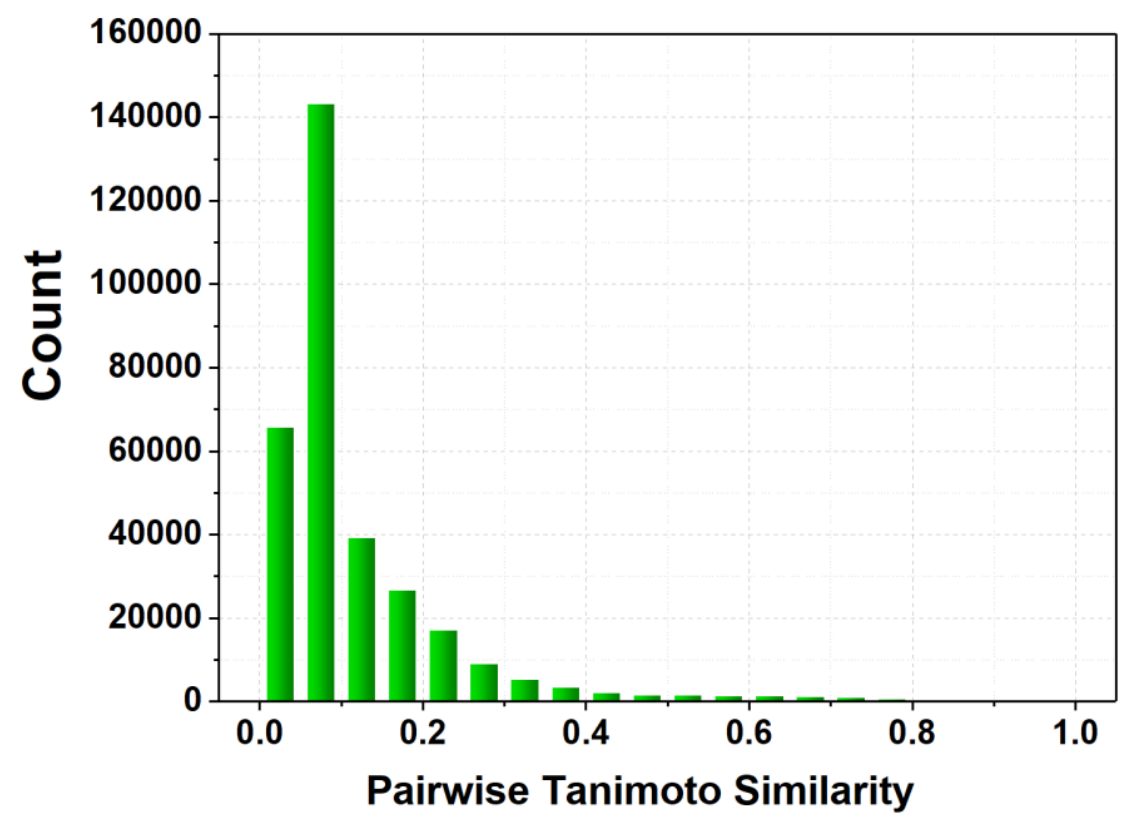

Figure S8. Histogram of the pairwise Tanimoto similarity between every two different compounds in our dataset (Dataset-Whole). 


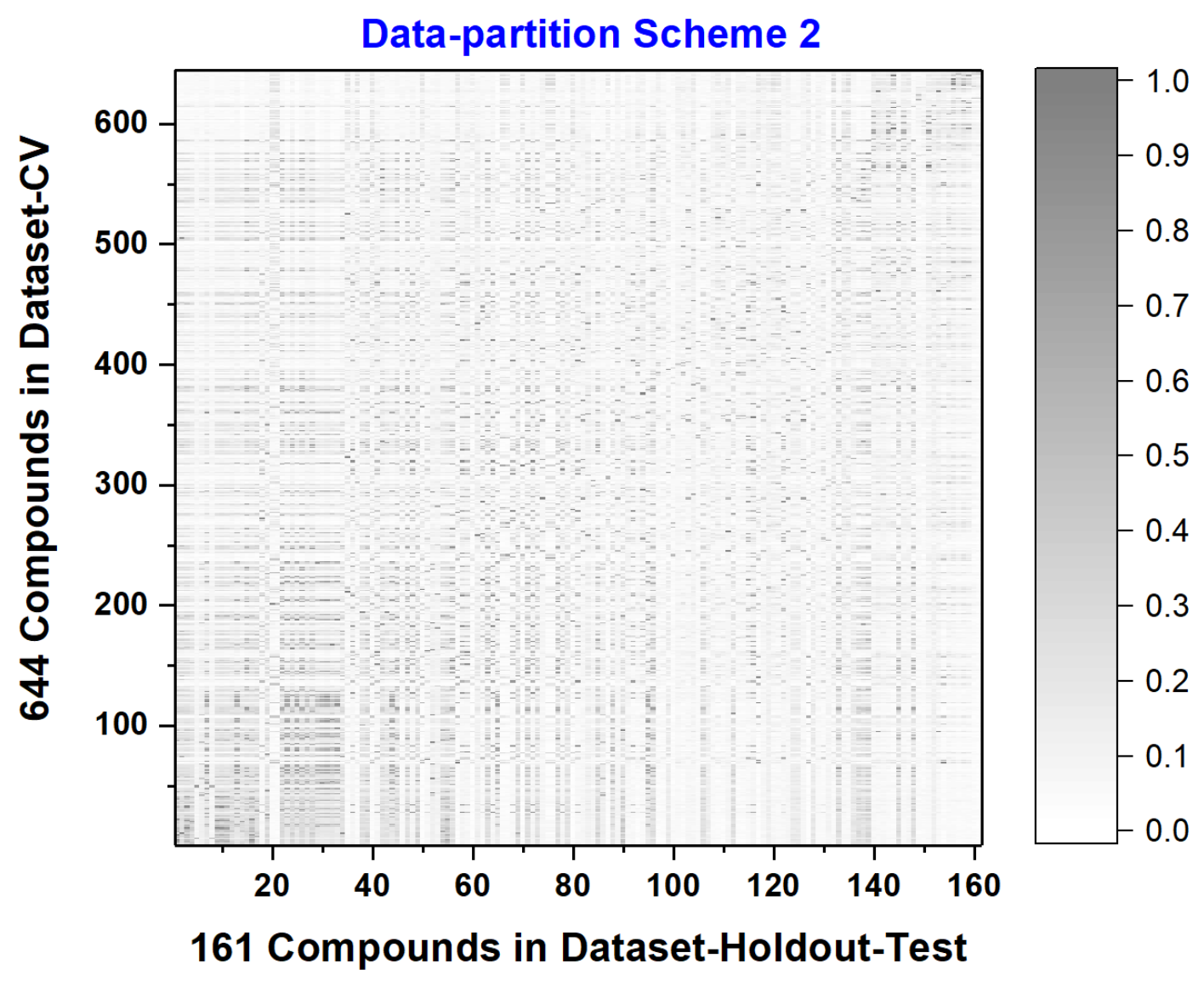

Figure S9. Tanimoto-similarity matrix between Dataset-CV (644 compounds) and Dataset-Holdout-Test (161 compounds) in data-partition scheme 2. Similarity is calculated based on 2048bit-ECFP6 fingerprint in our eHemolytic-Regression program. The average pairwise similarity in this matrix is 0.12 . Compounds in DatasetCV and Dataset-Holdout-Test are sorted in the ascending order of $\mathrm{HD}_{50}$. 


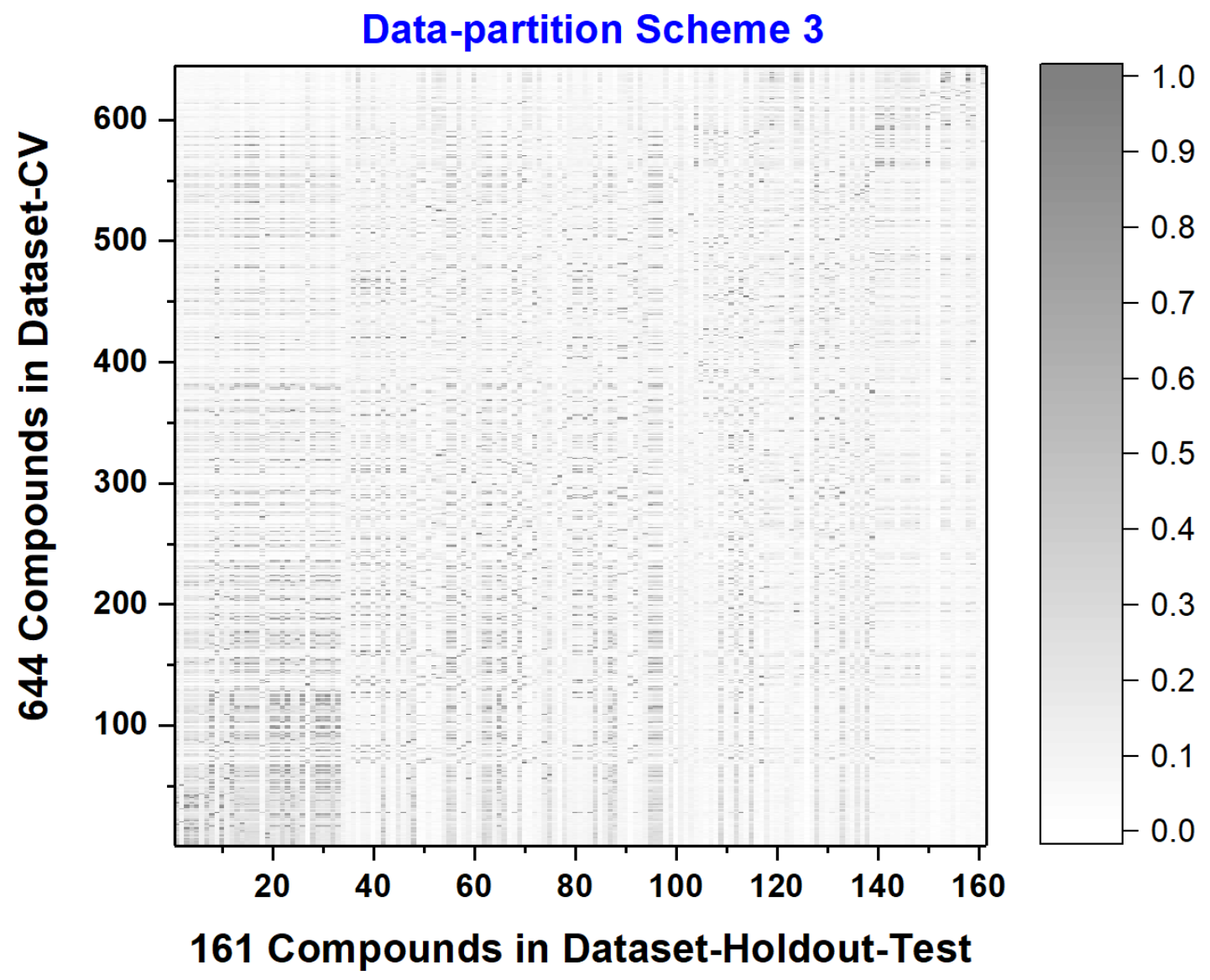

Figure S10. Tanimoto-similarity matrix between Dataset-CV (644 compounds) and Dataset-HoldoutTest (161 compounds) in data-partition scheme 3. Similarity is calculated based on 2048bit-ECFP6 fingerprint in our e-Hemolytic-Regression program. The average pairwise similarity in this matrix is 0.11. Compounds in Dataset-CV and Dataset-Holdout-Test are sorted in the ascending order of $\mathrm{HD}_{50}$. 


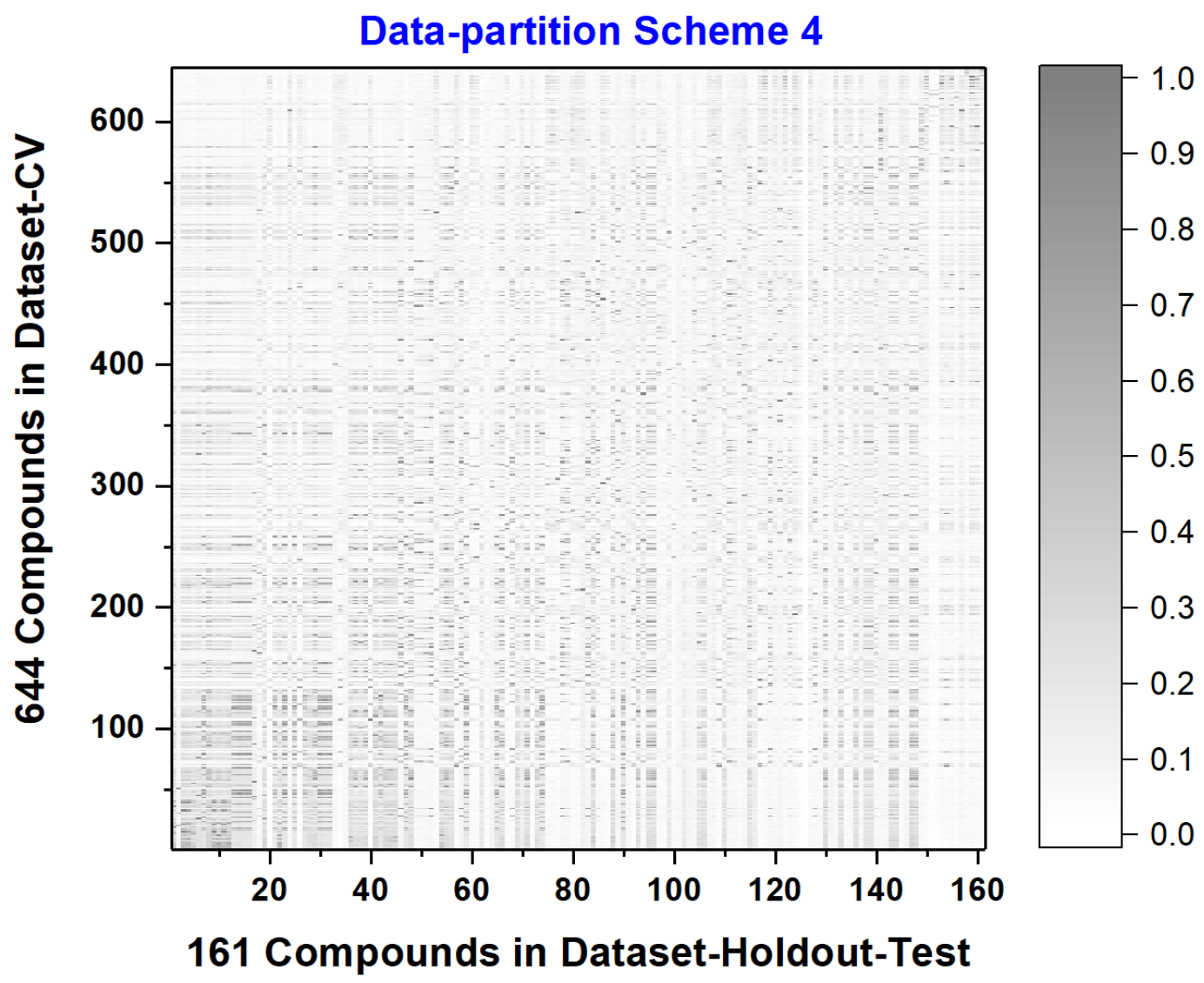

Figure S11. Tanimoto-similarity matrix between Dataset-CV (644 compounds) and Dataset-Holdout-Test (161 compounds) in data-partition scheme 4. Similarity is calculated based on 2048bit-ECFP6 fingerprint in our eHemolytic-Regression program. The average pairwise similarity in this matrix is 0.12 . Compounds in DatasetCV and Dataset-Holdout-Test are sorted in the ascending order of $\mathrm{HD}_{50}$. 


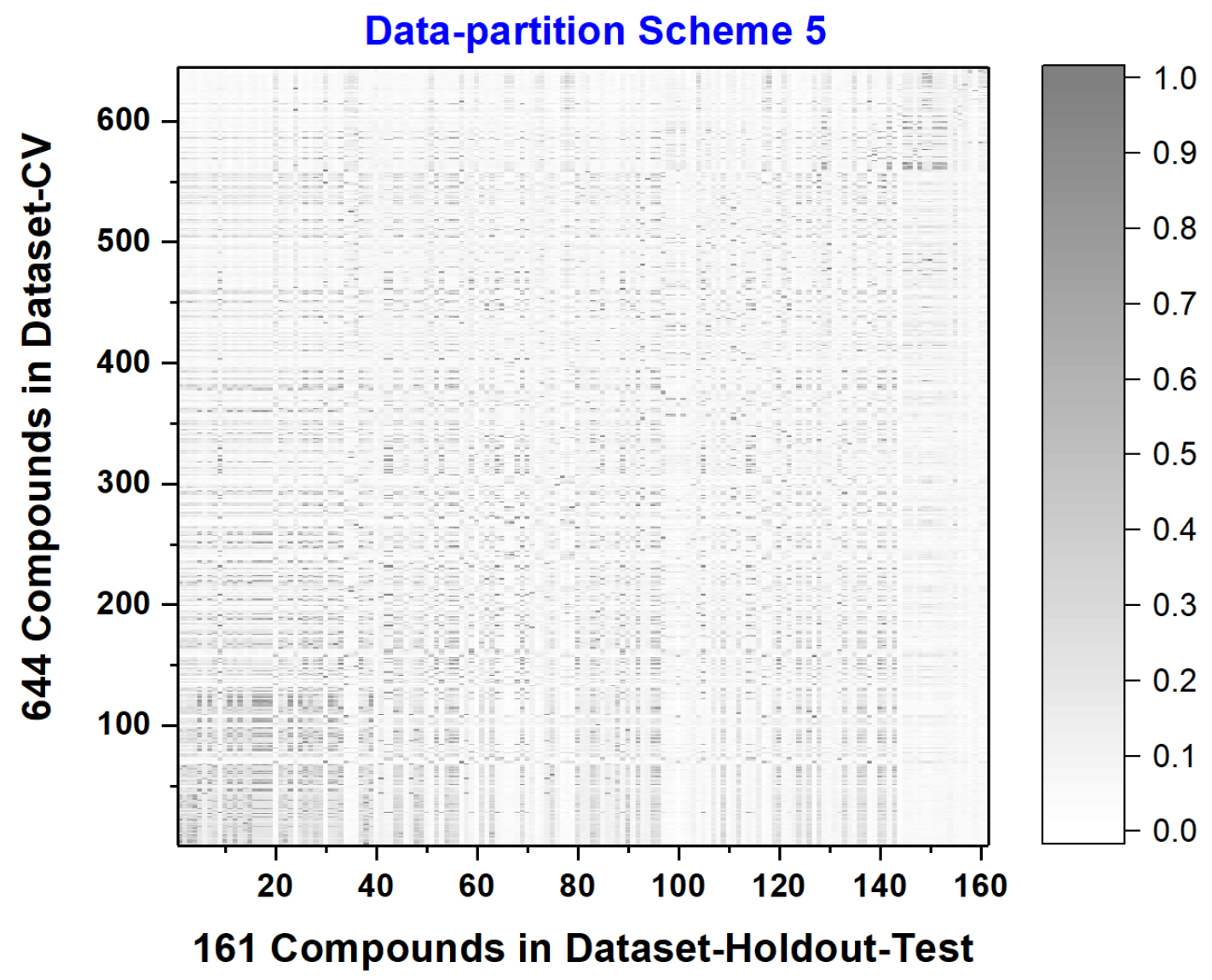

Figure S12. Tanimoto-similarity matrix between Dataset-CV (644 compounds) and DatasetHoldout-Test (161 compounds) in data-partition scheme 5. Similarity is calculated based on 2048bitECFP6 fingerprint in our e-Hemolytic-Regression program. The average pairwise similarity in this matrix is 0.12. Compounds in Dataset-CV and Dataset-Holdout-Test are sorted in the ascending order of $\mathrm{HD}_{50}$. 


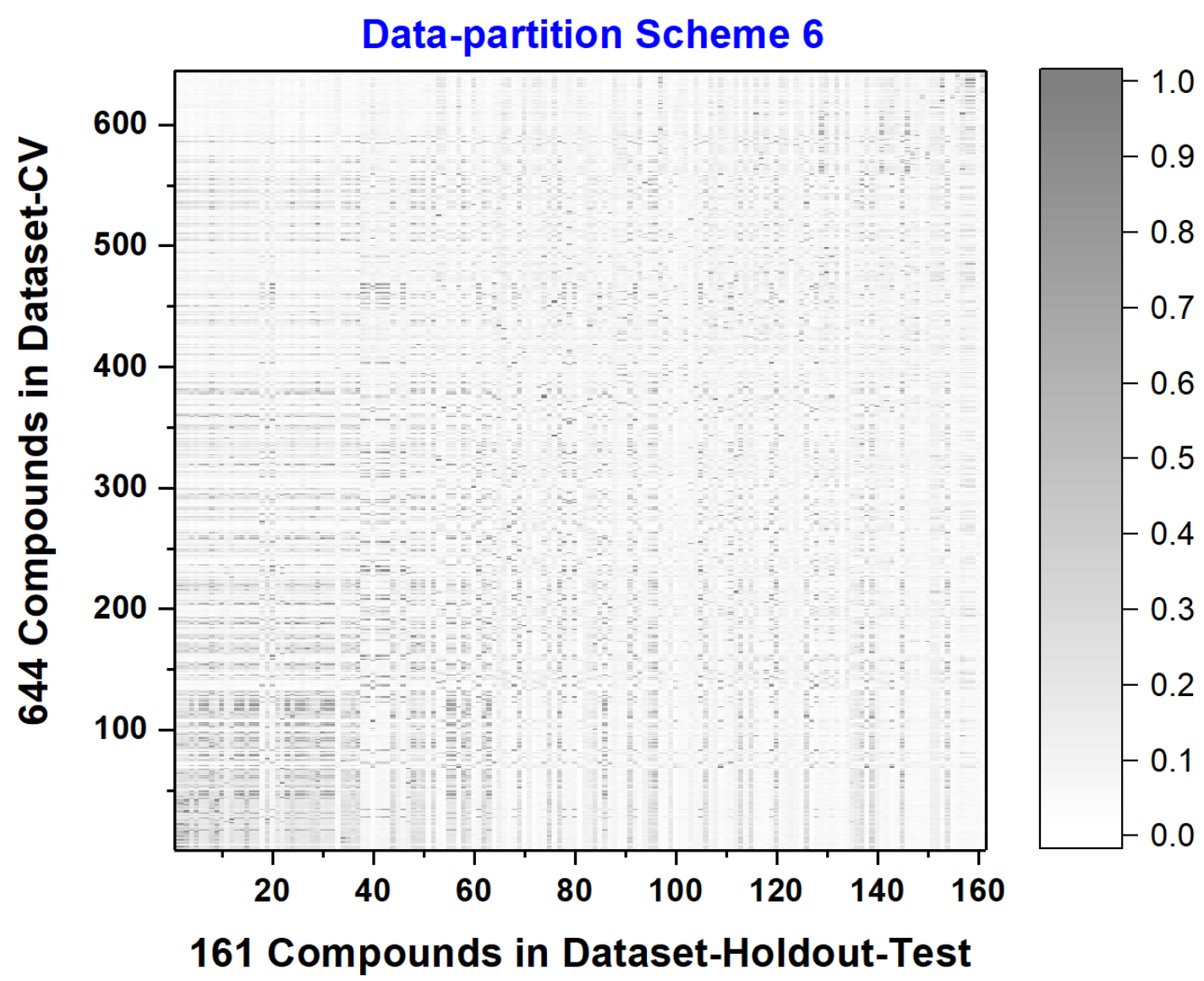

Figure S13. Tanimoto-similarity matrix between Dataset-CV (644 compounds) and Dataset-HoldoutTest (161 compounds) in data-partition scheme 6. Similarity is calculated based on 2048bit-ECFP6 fingerprint in our e-Hemolytic-Regression program. The average pairwise similarity in this matrix is 0.12. Compounds in Dataset-CV and Dataset-Holdout-Test are sorted in the ascending order of $\mathrm{HD}_{50}$. 


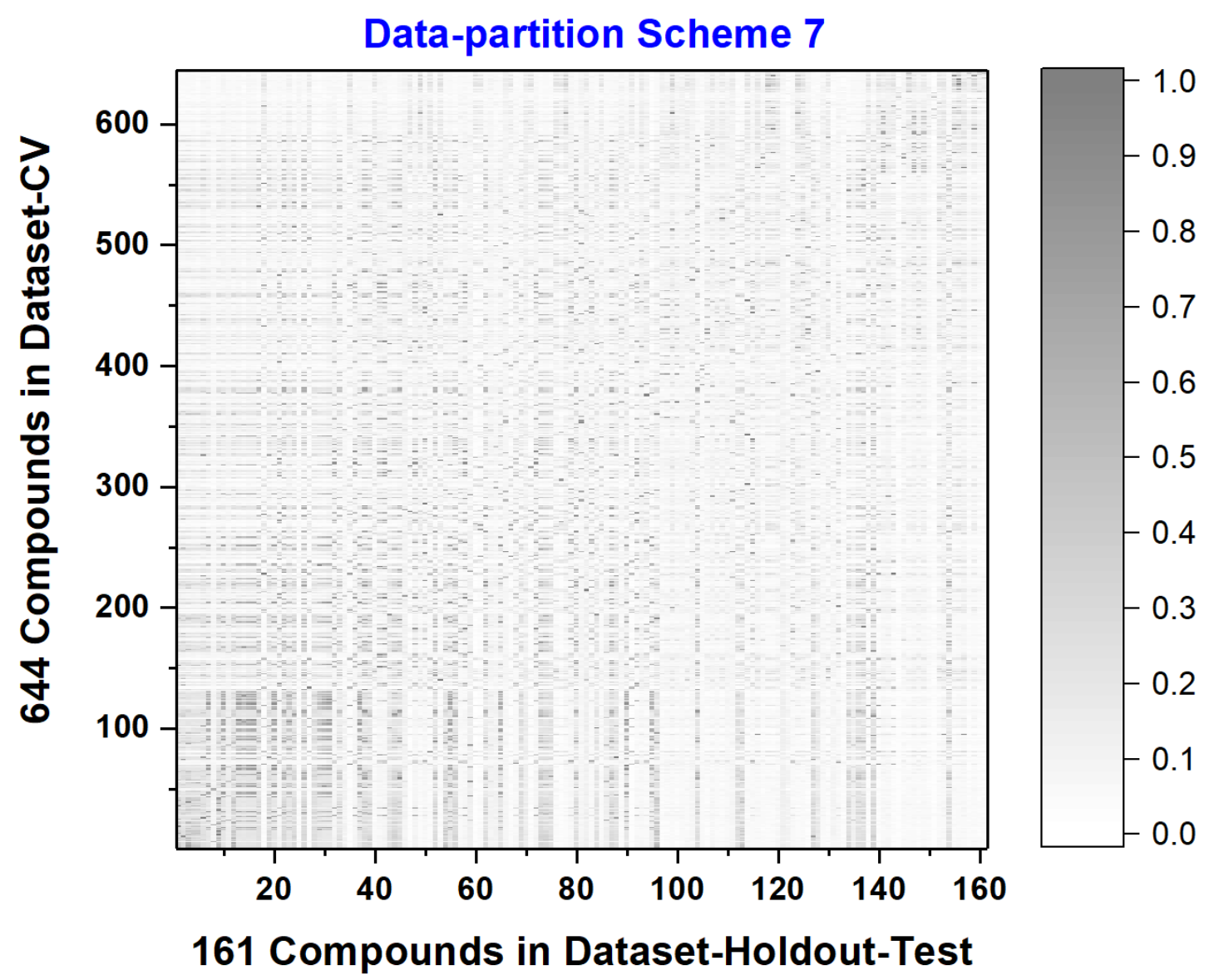

Figure S14. Tanimoto-similarity matrix between Dataset-CV (644 compounds) and Dataset-HoldoutTest (161 compounds) in data-partition scheme 7. Similarity is calculated based on 2048bit-ECFP6 fingerprint in our e-Hemolytic-Regression program. The average pairwise similarity in this matrix is 0.11. Compounds in Dataset-CV and Dataset-Holdout-Test are sorted in the ascending order of $\mathrm{HD}_{50}$. 


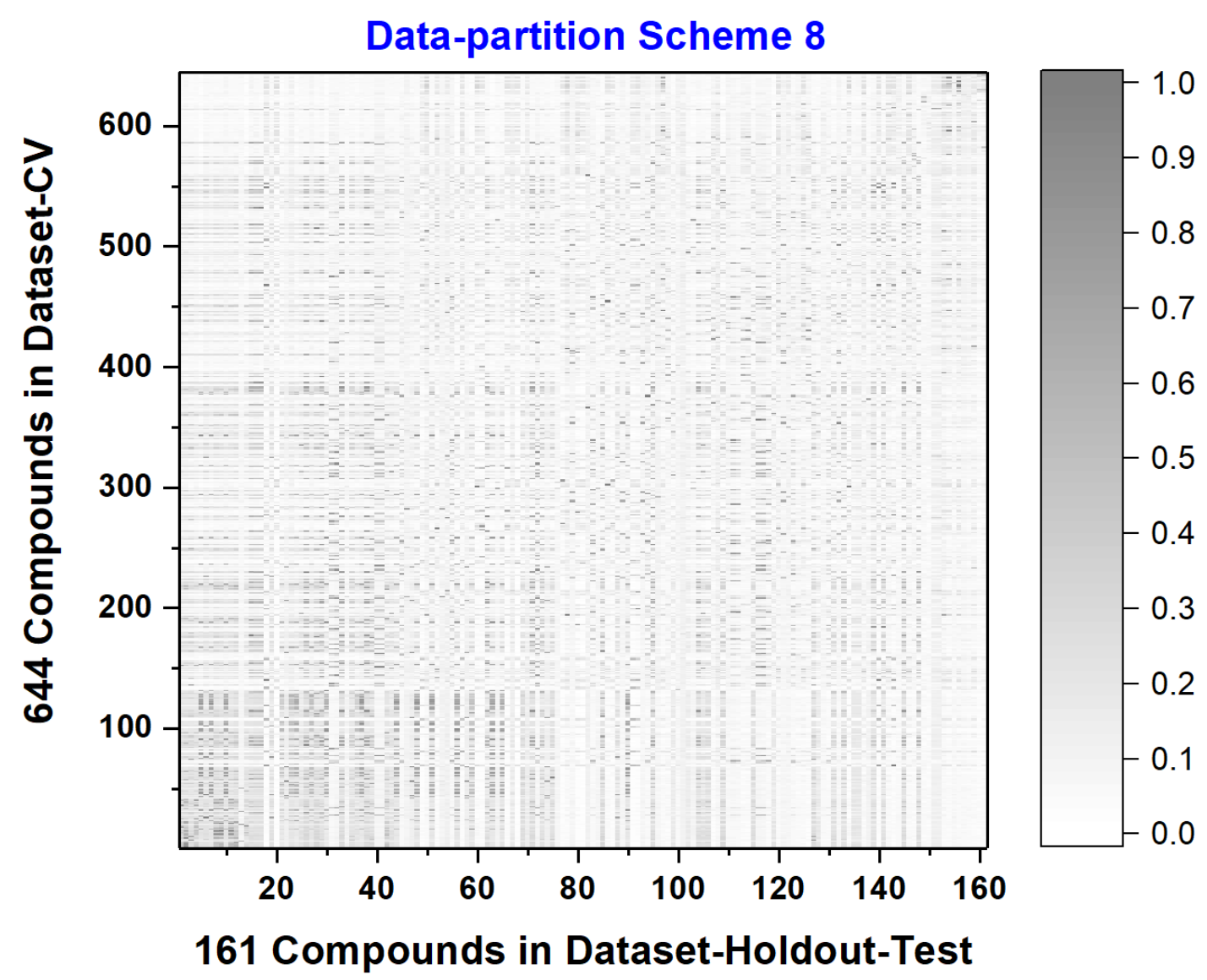

Figure S15. Tanimoto-similarity matrix between Dataset-CV (644 compounds) and DatasetHoldout-Test (161 compounds) in data-partition scheme 8. Similarity is calculated based on 2048bitECFP6 fingerprint in our e-Hemolytic-Regression program. The average pairwise similarity in this matrix is 0.12. Compounds in Dataset-CV and Dataset-Holdout-Test are sorted in the ascending order of $\mathrm{HD}_{50}$. 


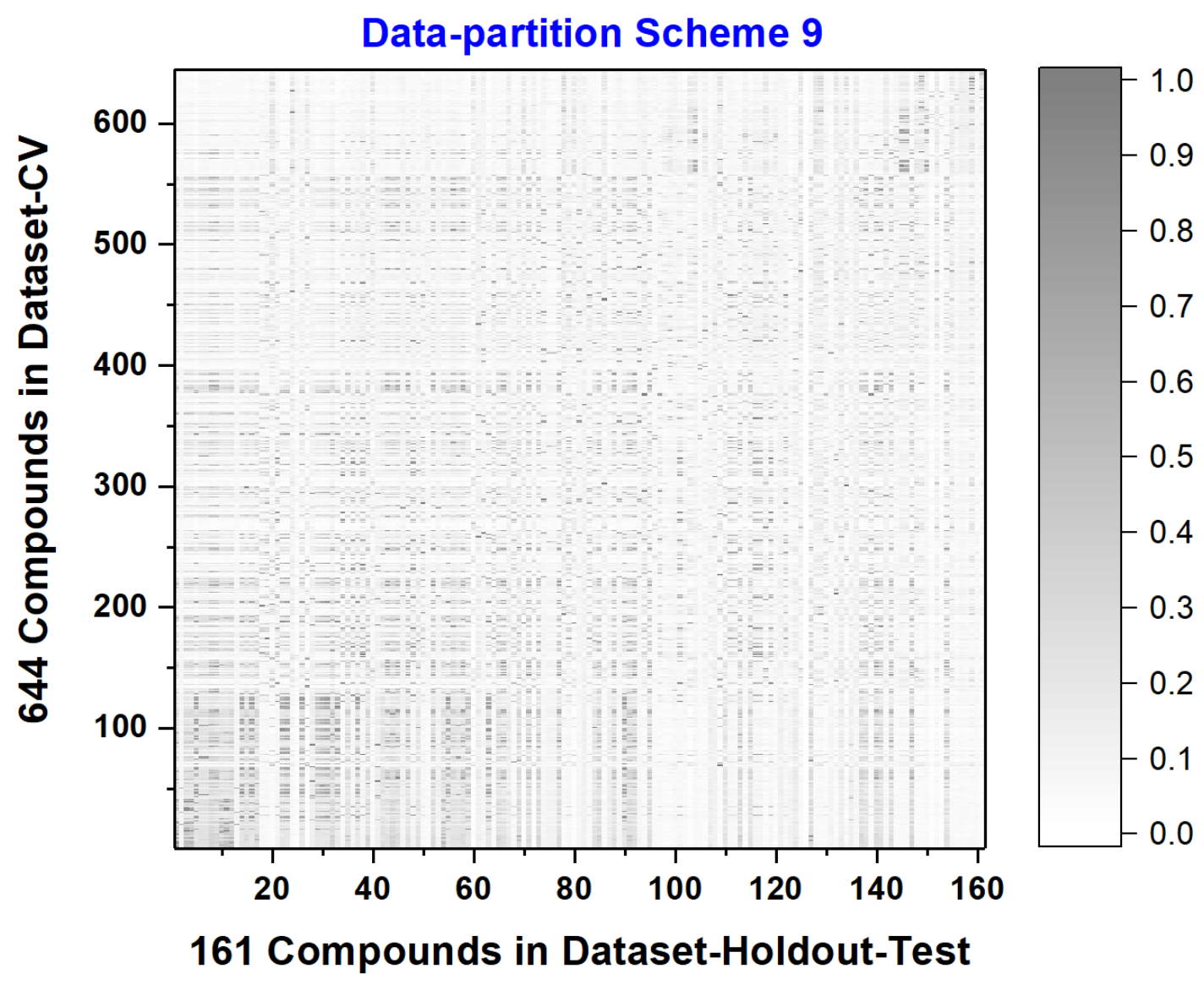

Figure S16. Tanimoto-similarity matrix between Dataset-CV (644 compounds) and Dataset-HoldoutTest (161 compounds) in data-partition scheme 9. Similarity is calculated based on 2048bit-ECFP6 fingerprint in our e-Hemolytic-Regression program. The average pairwise similarity in this matrix is 0.12. Compounds in Dataset-CV and Dataset-Holdout-Test are sorted in the ascending order of $\mathrm{HD}_{50}$. 


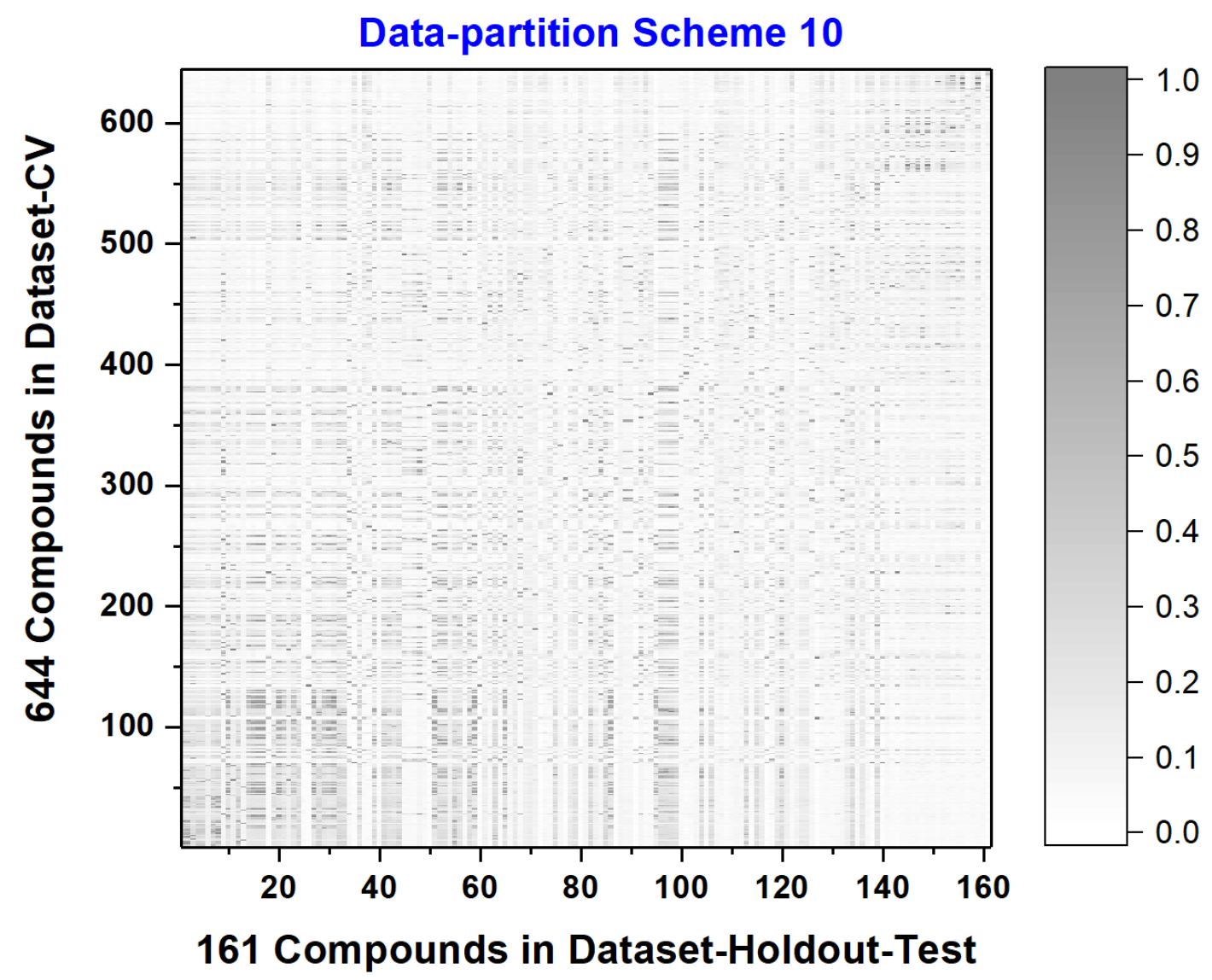

Figure S17. Tanimoto-similarity matrix between Dataset-CV (644 compounds) and DatasetHoldout-Test (161 compounds) in data-partition scheme 10. Similarity is calculated based on 2048bitECFP6 fingerprint in our e-Hemolytic-Regression program. The average pairwise similarity in this matrix is 0.11. Compounds in Dataset-CV and Dataset-Holdout-Test are sorted in the ascending order of $\mathrm{HD}_{50}$. 


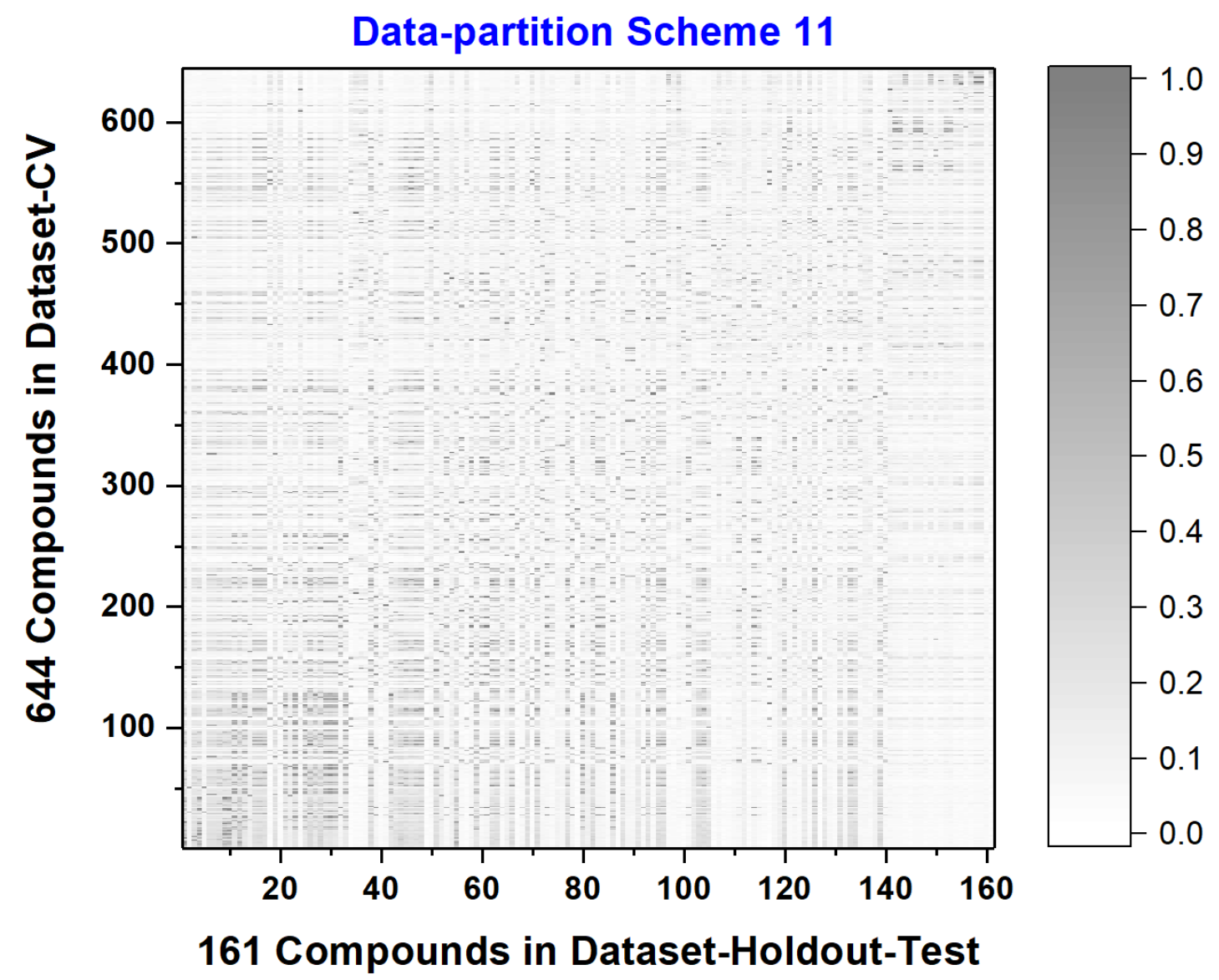

Figure S18. Tanimoto-similarity matrix between Dataset-CV (644 compounds) and Dataset-HoldoutTest (161 compounds) in data-partition scheme 11. Similarity is calculated based on 2048bit-ECFP6 fingerprint in our e-Hemolytic-Regression program. The average pairwise similarity in this matrix is 0.12. Compounds in Dataset-CV and Dataset-Holdout-Test are sorted in the ascending order of $\mathrm{HD}_{50}$. 


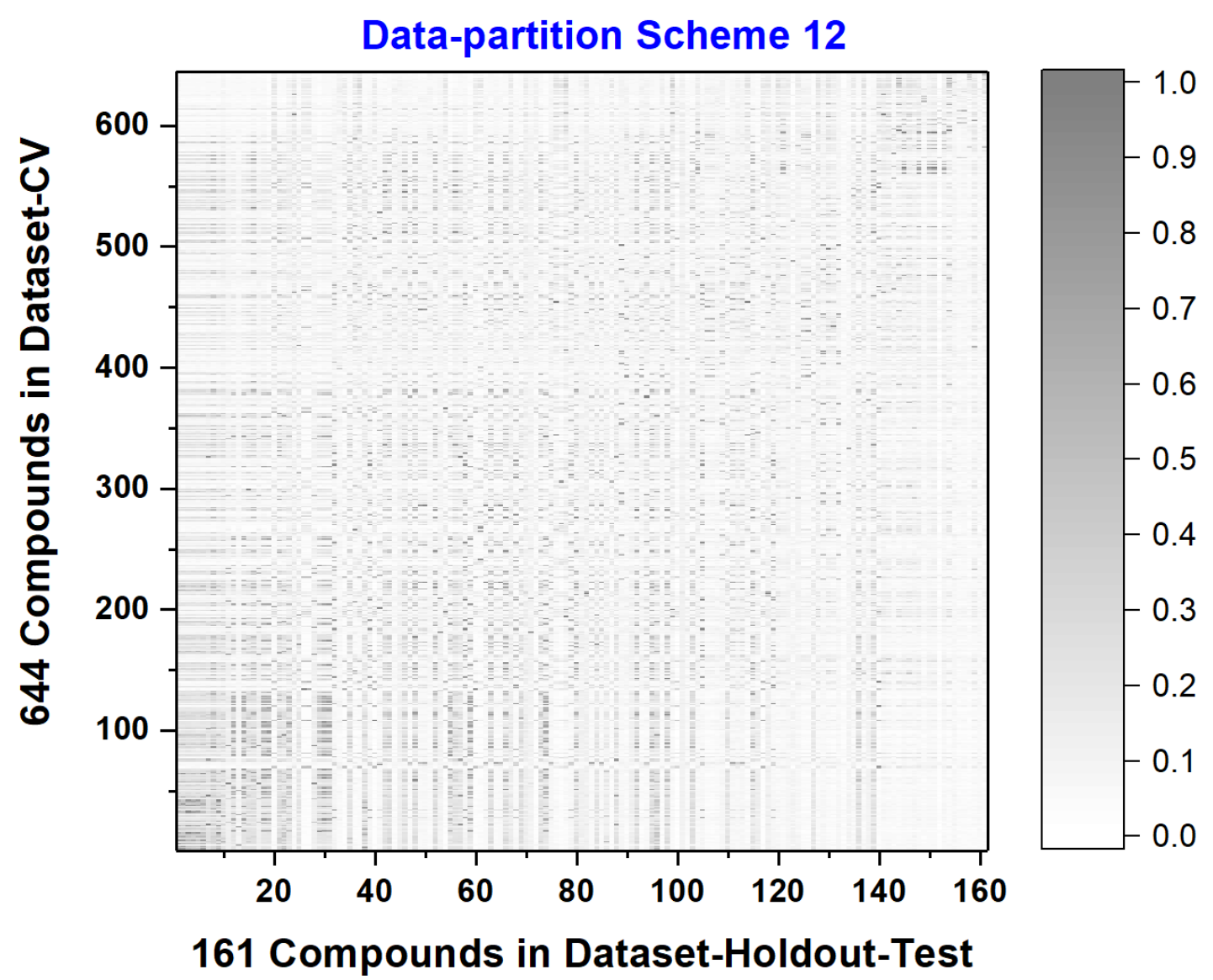

Figure S19. Tanimoto-similarity matrix between Dataset-CV (644 compounds) and Dataset-HoldoutTest (161 compounds) in data-partition scheme 12. Similarity is calculated based on 2048bit-ECFP6 fingerprint in our e-Hemolytic-Regression program. The average pairwise similarity in this matrix is 0.11. Compounds in Dataset-CV and Dataset-Holdout-Test are sorted in the ascending order of $\mathrm{HD}_{50}$. 


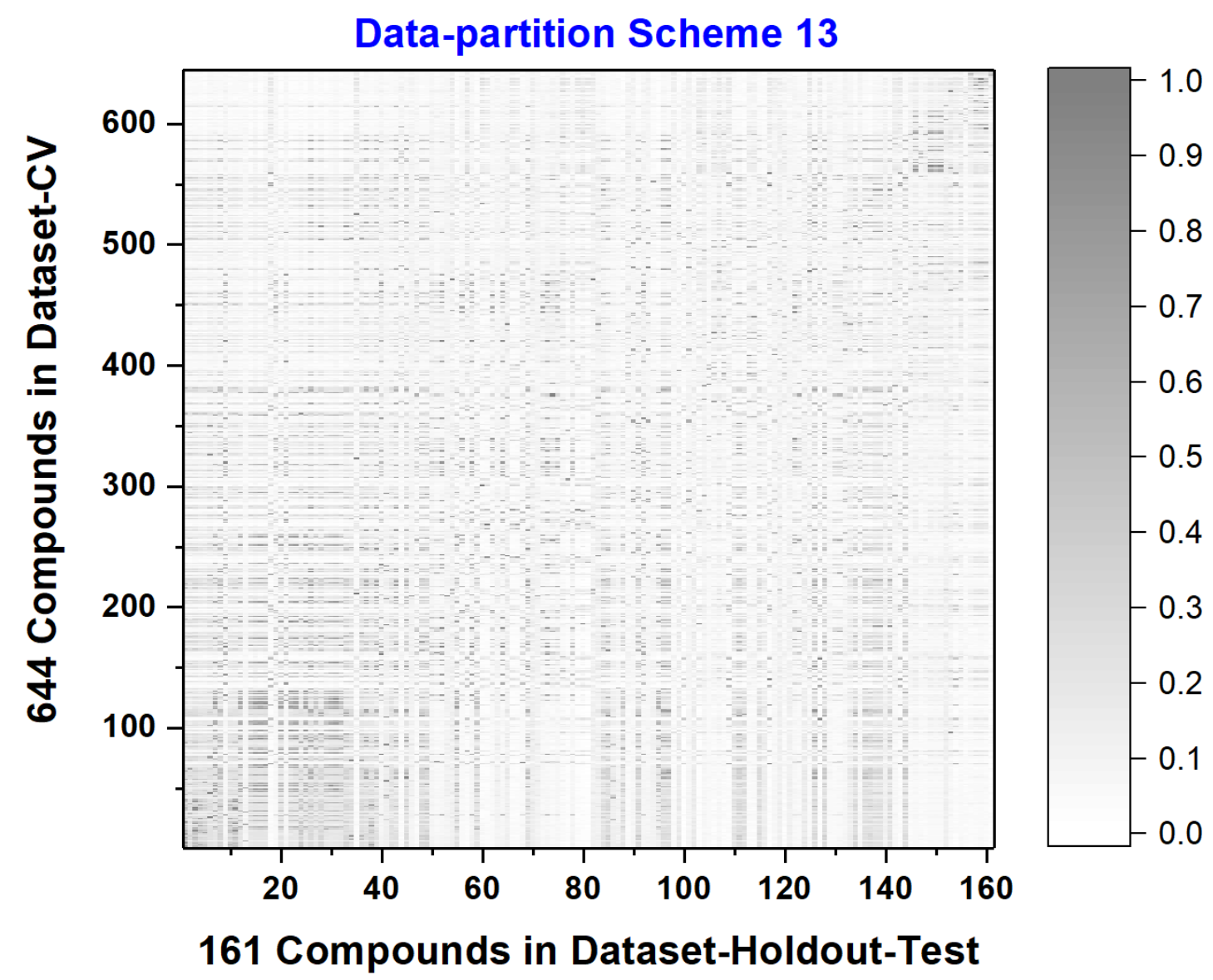

Figure S20. Tanimoto-similarity matrix between Dataset-CV (644 compounds) and Dataset-HoldoutTest (161 compounds) in data-partition scheme 13. Similarity is calculated based on 2048bit-ECFP6 fingerprint in our e-Hemolytic-Regression program. The average pairwise similarity in this matrix is 0.12. Compounds in Dataset-CV and Dataset-Holdout-Test are sorted in the ascending order of $\mathrm{HD}_{50}$. 


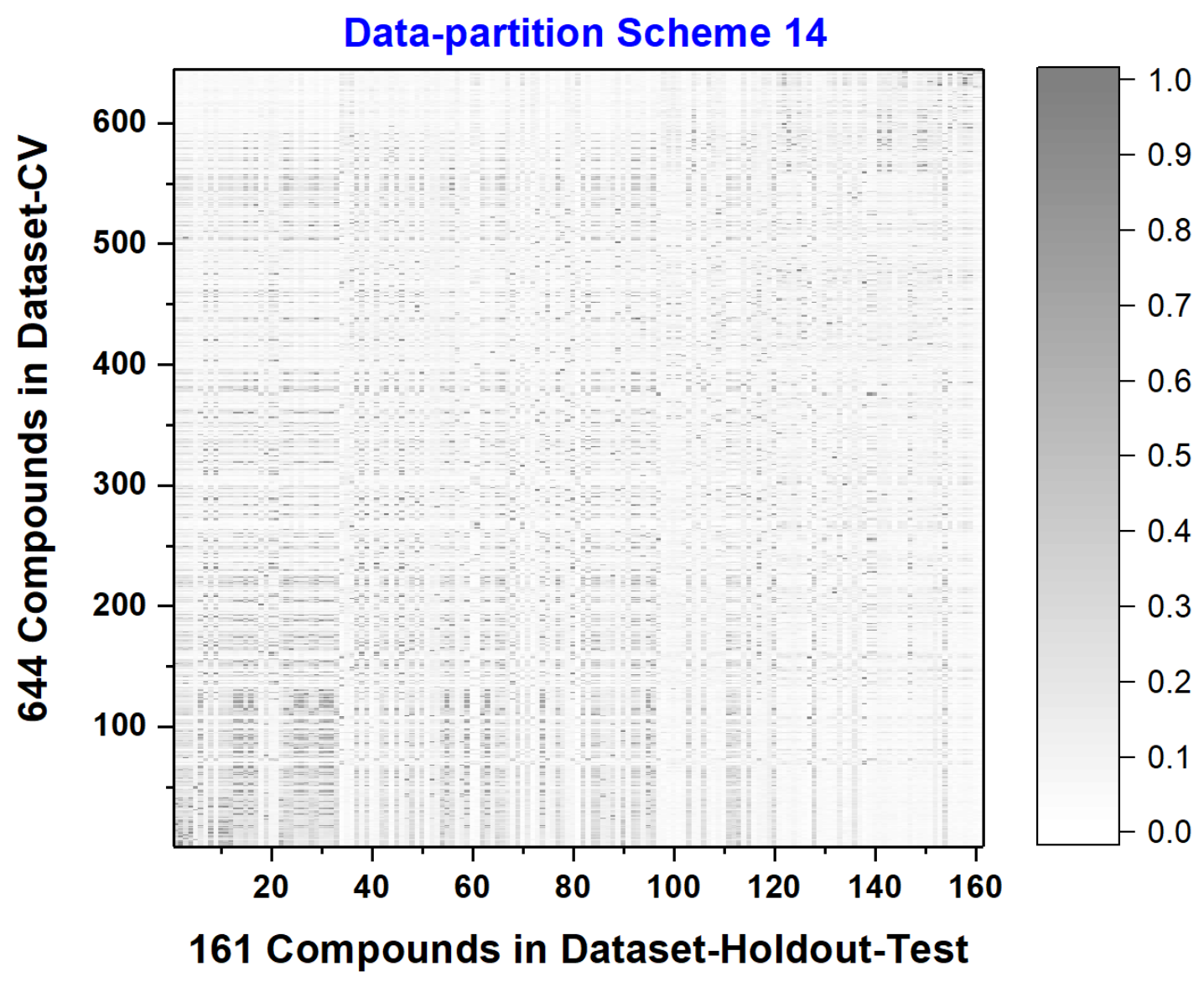

Figure S21. Tanimoto-similarity matrix between Dataset-CV (644 compounds) and Dataset-HoldoutTest (161 compounds) in data-partition scheme 14. Similarity is calculated based on 2048bit-ECFP6 fingerprint in our e-Hemolytic-Regression program. The average pairwise similarity in this matrix is 0.12. Compounds in Dataset-CV and Dataset-Holdout-Test are sorted in the ascending order of $\mathrm{HD}_{50}$. 


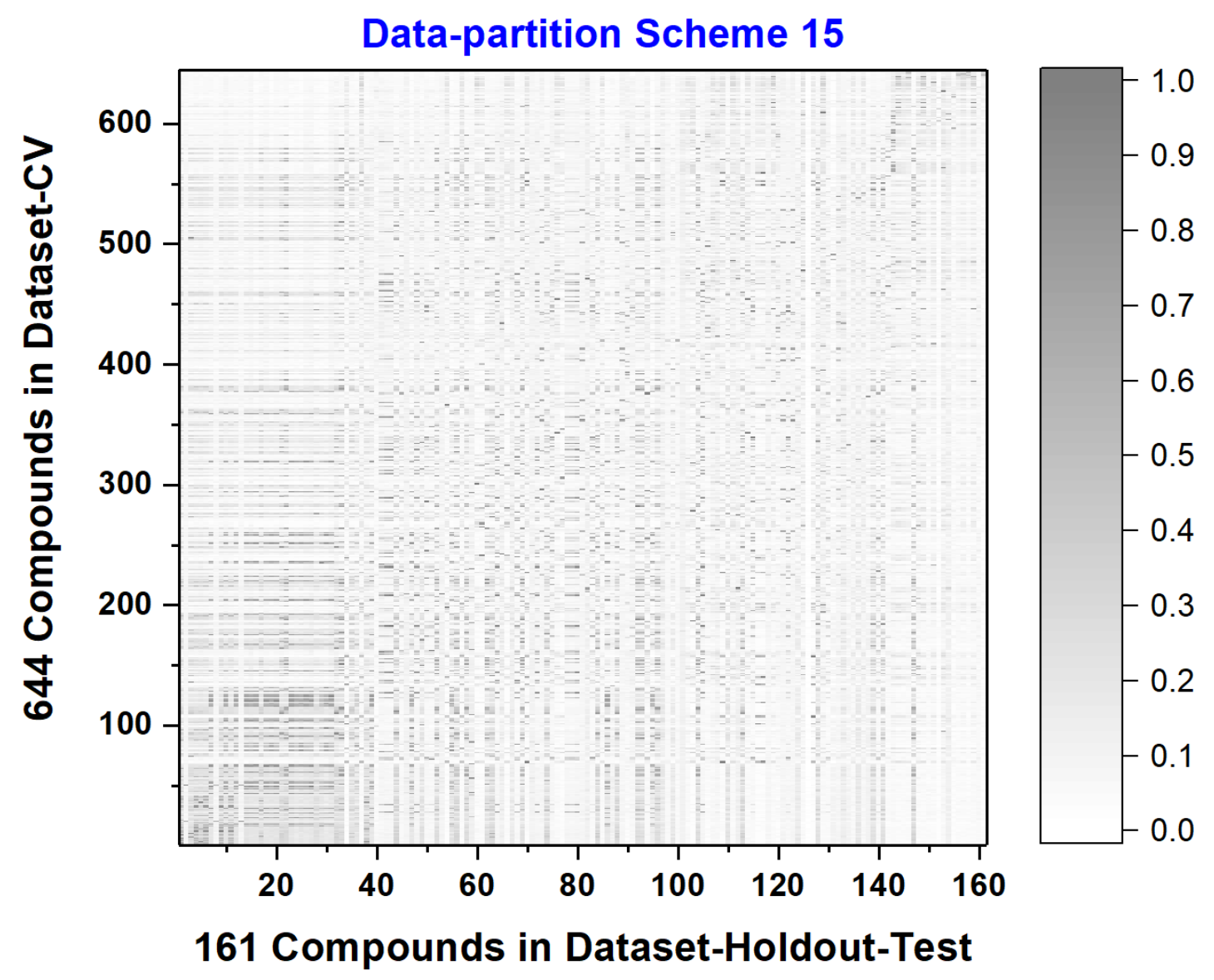

Figure S22. Tanimoto-similarity matrix between Dataset-CV (644 compounds) and Dataset-HoldoutTest (161 compounds) in data-partition scheme 15. Similarity is calculated based on 2048bit-ECFP6 fingerprint in our e-Hemolytic-Regression program. The average pairwise similarity in this matrix is 0.12. Compounds in Dataset-CV and Dataset-Holdout-Test are sorted in the ascending order of $\mathrm{HD}_{50}$. 


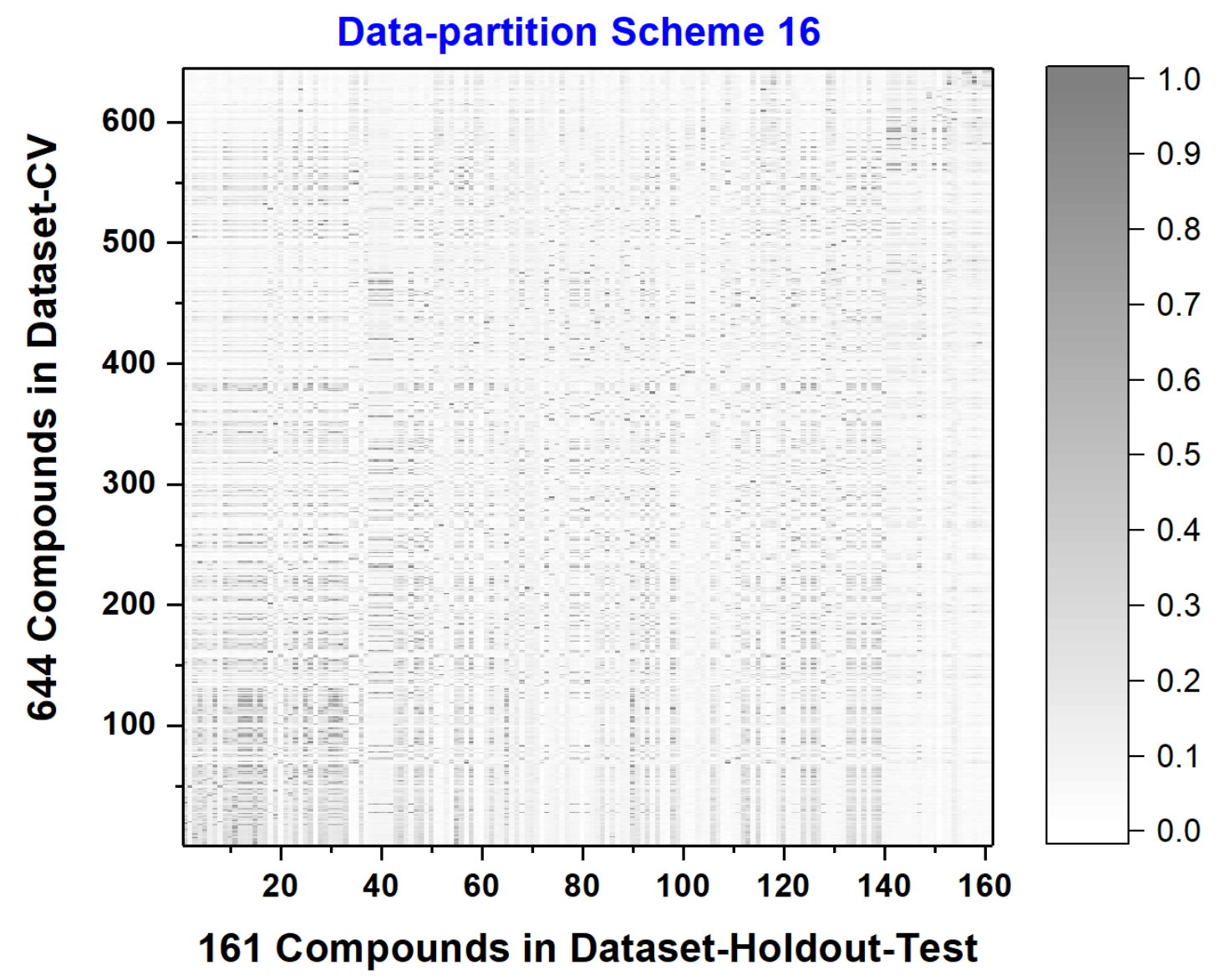

Figure S23. Tanimoto-similarity matrix between Dataset-CV (644 compounds) and Dataset-HoldoutTest (161 compounds) in data-partition scheme 16. Similarity is calculated based on 2048bit-ECFP6 fingerprint in our e-Hemolytic-Regression program. The average pairwise similarity in this matrix is 0.12. Compounds in Dataset-CV and Dataset-Holdout-Test are sorted in the ascending order of $\mathrm{HD}_{50}$. 


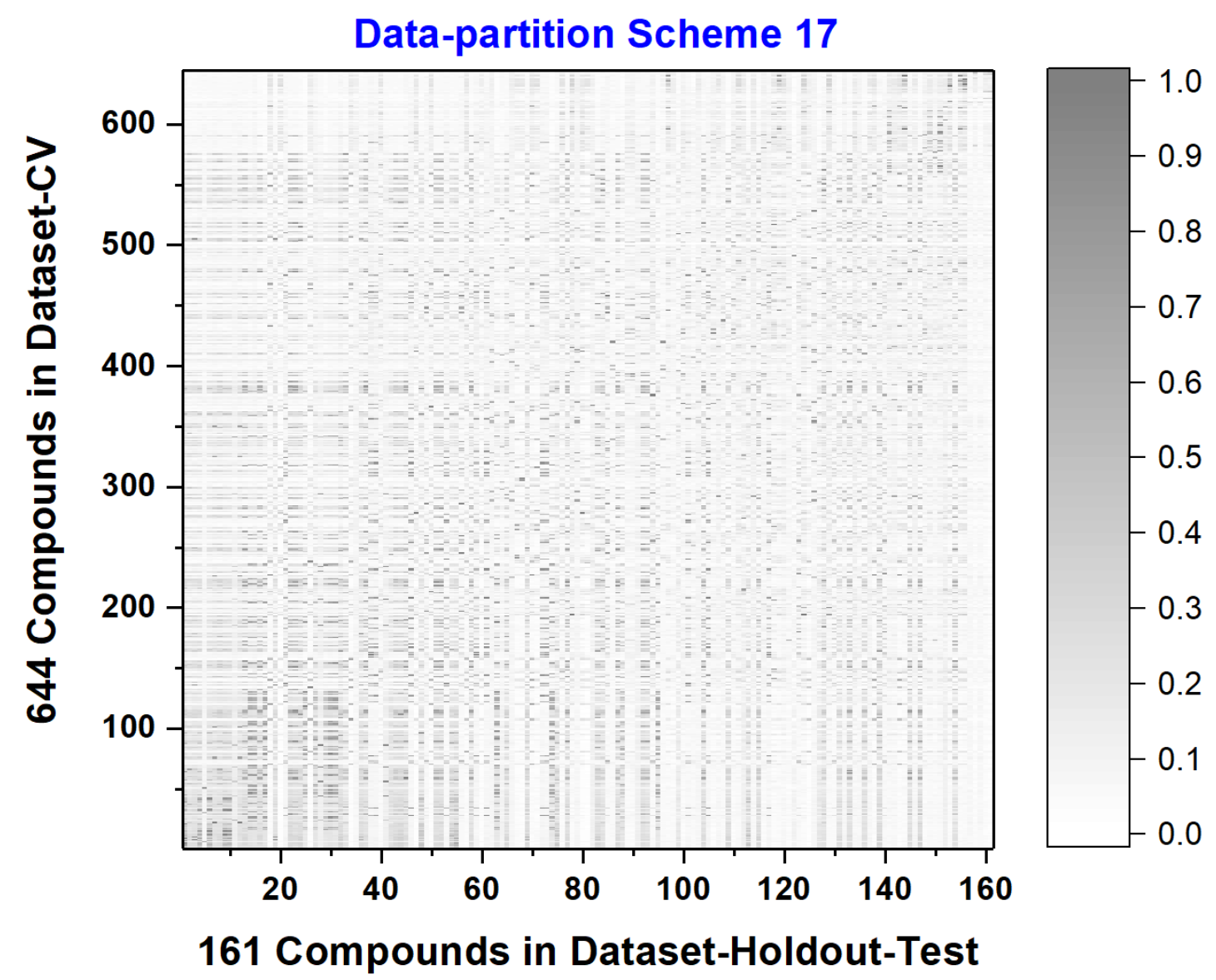

Figure S24. Tanimoto-similarity matrix between Dataset-CV (644 compounds) and Dataset-HoldoutTest (161 compounds) in data-partition scheme 17. Similarity is calculated based on 2048bit-ECFP6 fingerprint in our e-Hemolytic-Regression program. The average pairwise similarity in this matrix is 0.12. Compounds in Dataset-CV and Dataset-Holdout-Test are sorted in the ascending order of $\mathrm{HD}_{50}$. 


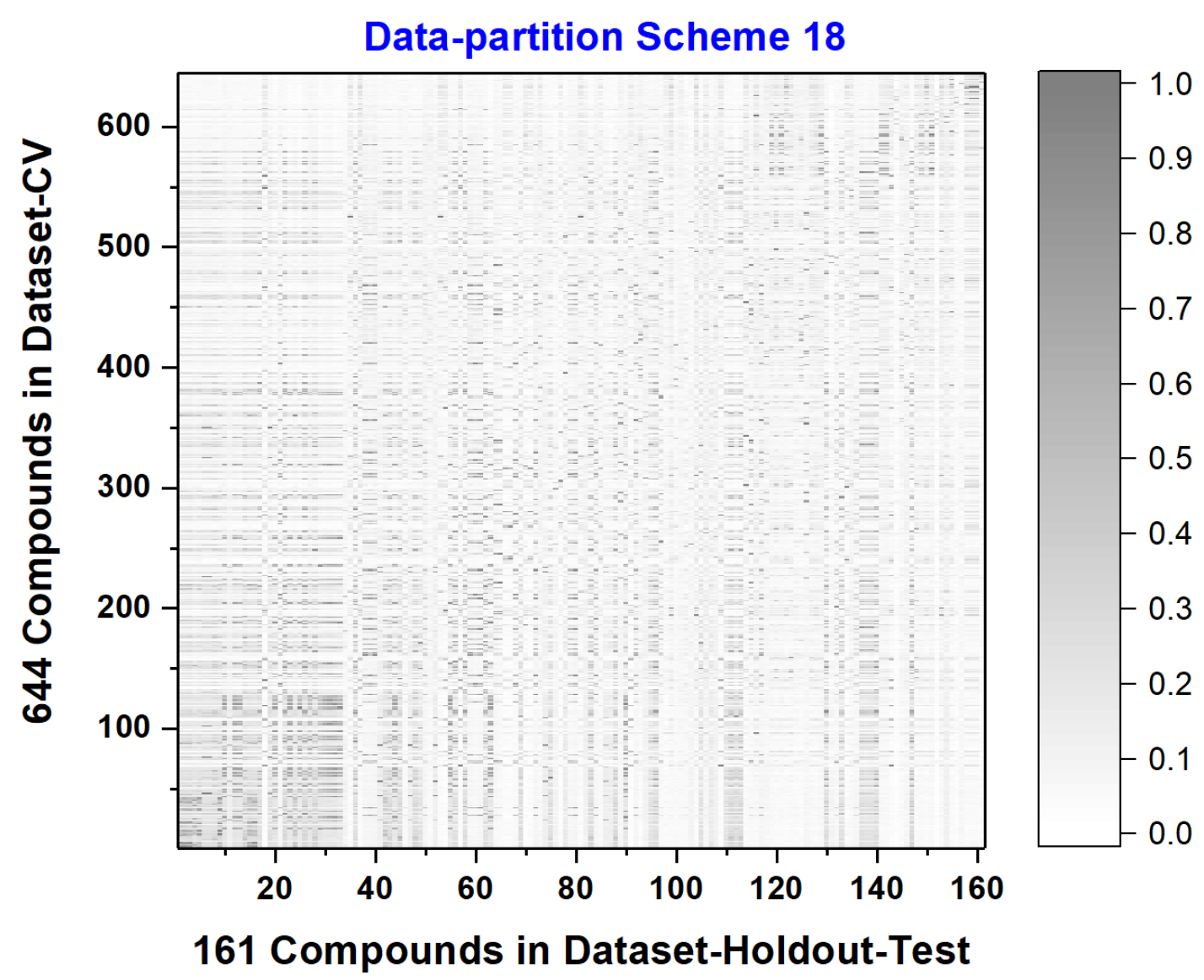

Figure S25. Tanimoto-similarity matrix between Dataset-CV (644 compounds) and Dataset-HoldoutTest (161 compounds) in data-partition scheme 18. Similarity is calculated based on 2048bit-ECFP6 fingerprint in our e-Hemolytic-Regression program. The average pairwise similarity in this matrix is 0.12. Compounds in Dataset-CV and Dataset-Holdout-Test are sorted in the ascending order of $\mathrm{HD}_{50}$. 


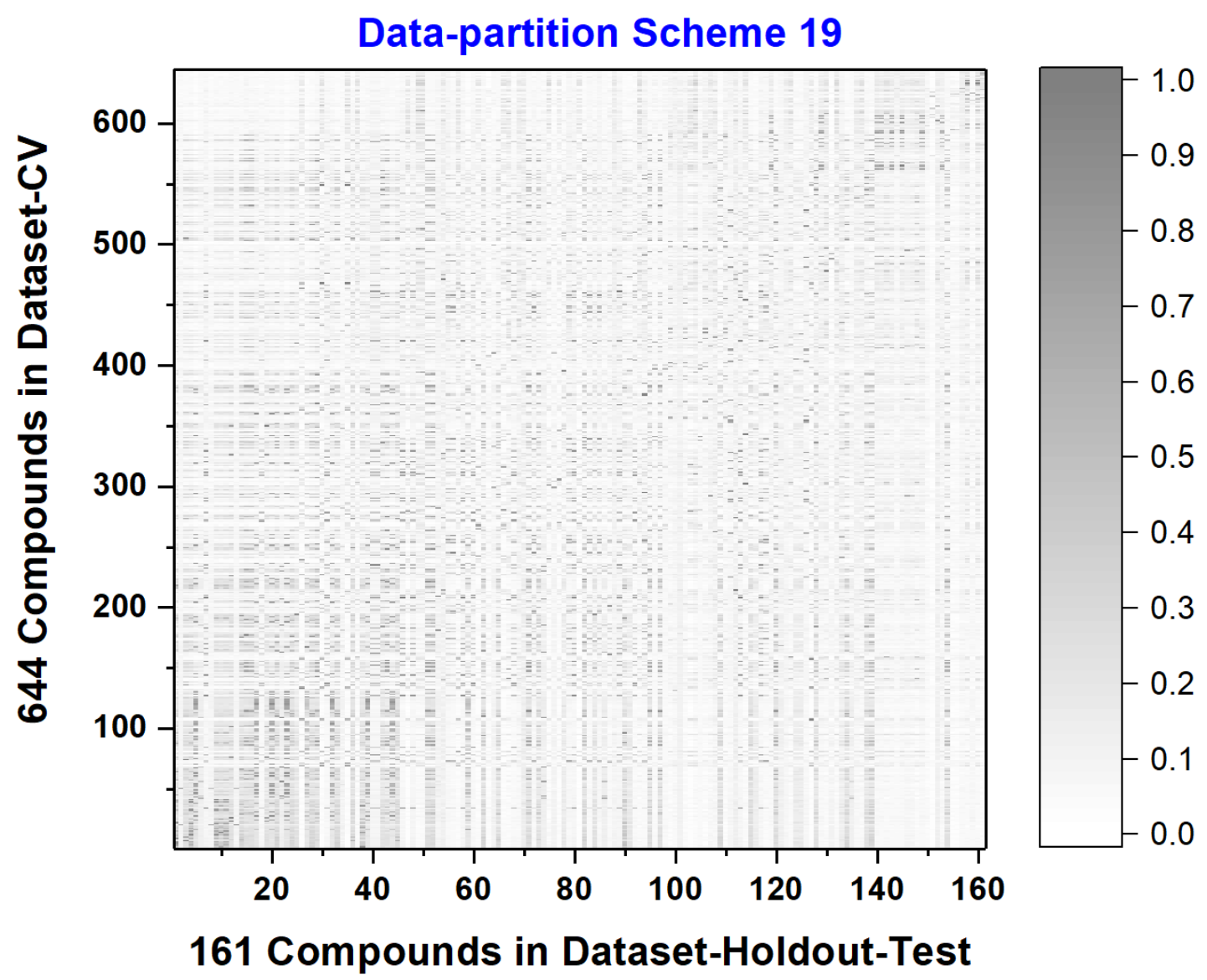

Figure S26. Tanimoto-similarity matrix between Dataset-CV (644 compounds) and Dataset-HoldoutTest (161 compounds) in data-partition scheme 19. Similarity is calculated based on 2048bit-ECFP6 fingerprint in our e-Hemolytic-Regression program. The average pairwise similarity in this matrix is 0.11. Compounds in Dataset-CV and Dataset-Holdout-Test are sorted in the ascending order of $\mathrm{HD}_{50}$. 


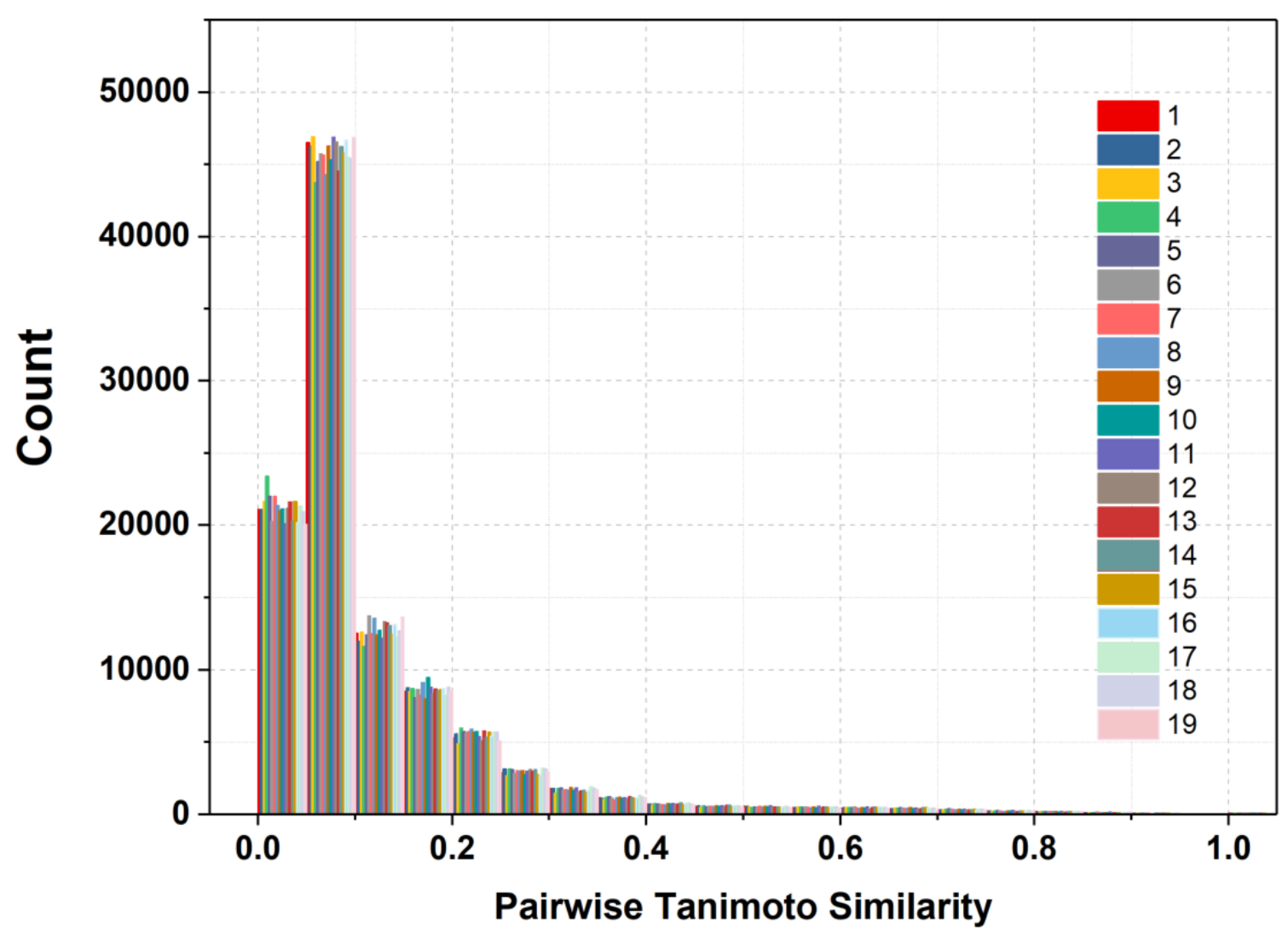

Figure S27. Histograms of the pairwise Tanimoto similarity between Dataset-CV (664 compounds) and DatasetHoldout-Test (161 compounds) for 19 data-partition schemes. 


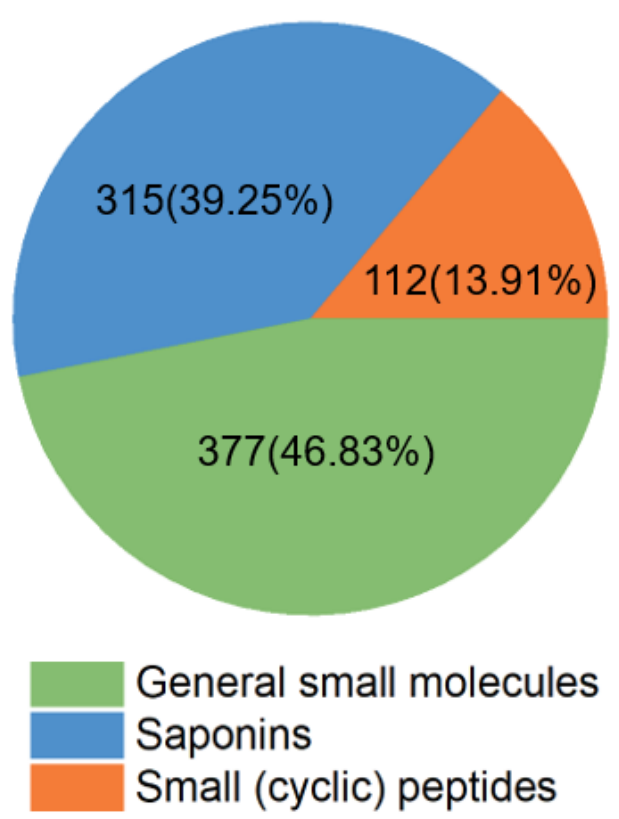

Figure S28. Distribution of different chemotypes in our quantitative hemolytic toxicity dataset. 


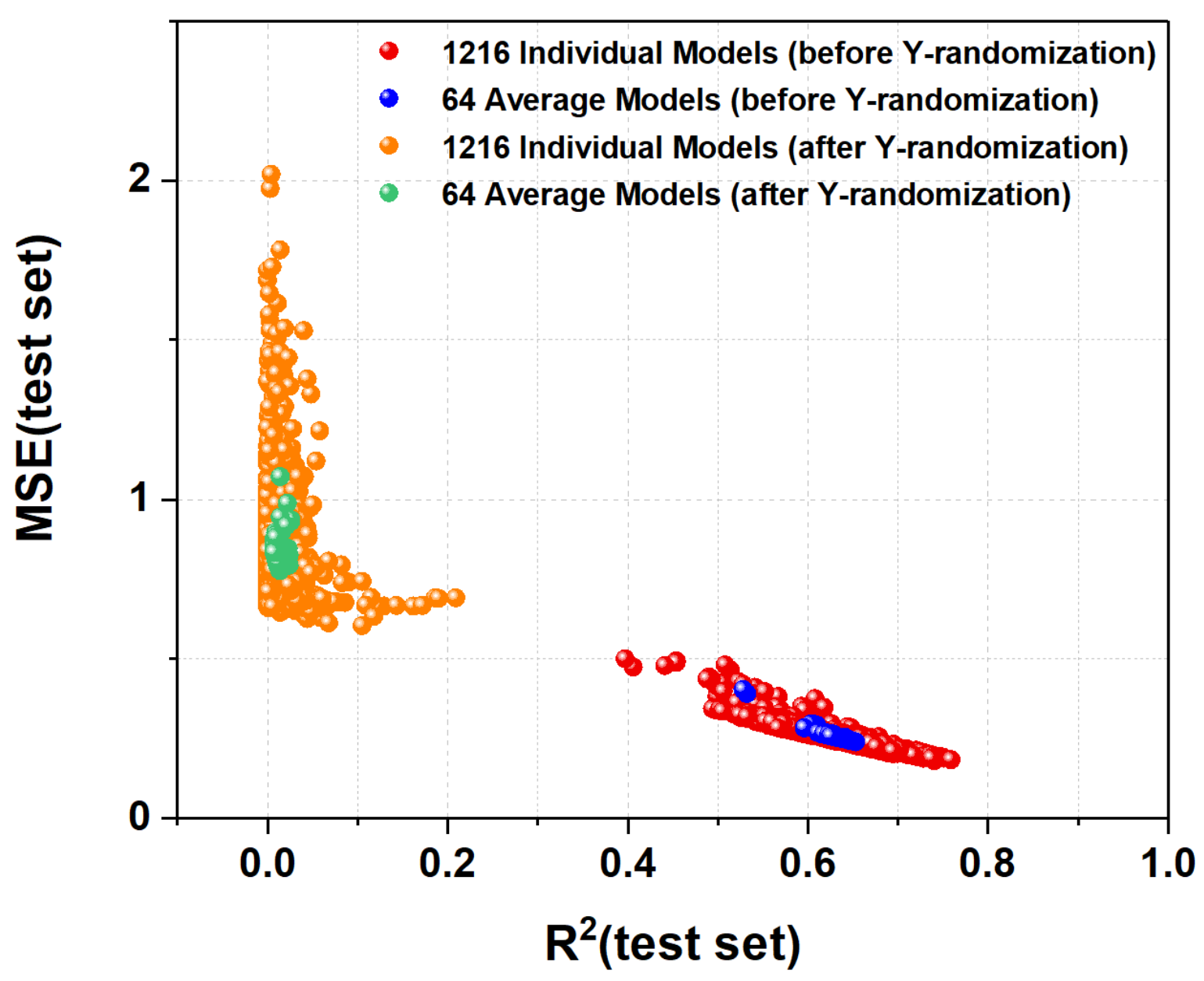

Figure S29. Scatter plot of $\mathrm{R}^{2}$ (holdout test set) vs. MSE(holdout test set) for the regression models including all the individual and average models before/after $\mathrm{Y}$-randomization. 


\section{Consensus/Average Model}

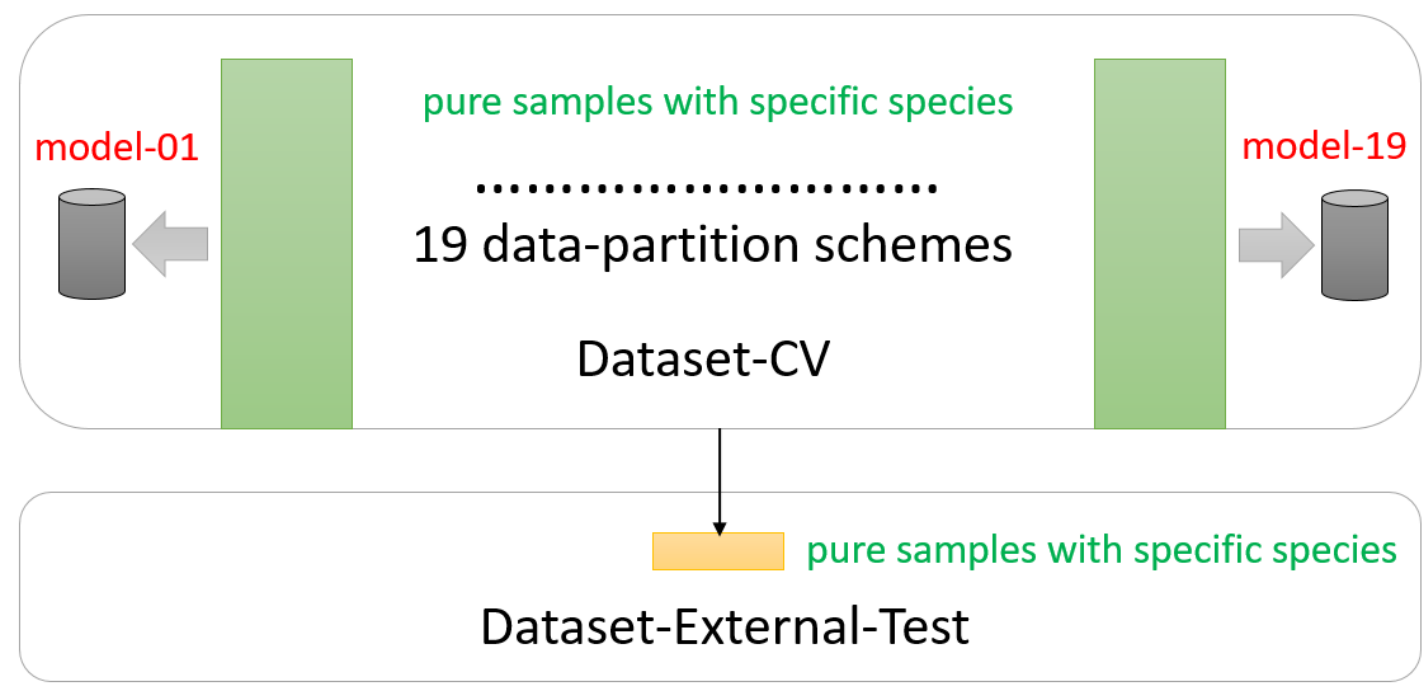

Requires sufficient and pure samples for each species in Dataset-CV and Dataset-External-Test

Figure S30. Protocol used to train and validate the models based on the pure samples with each species. Yellow rectangle refers to Dataset-External-Test; Green rectangles refer to Dataset-CV from 19 different data-splitting schemes that afford 19 individual models (model-01, model-02,.., model-18 and model-19). 


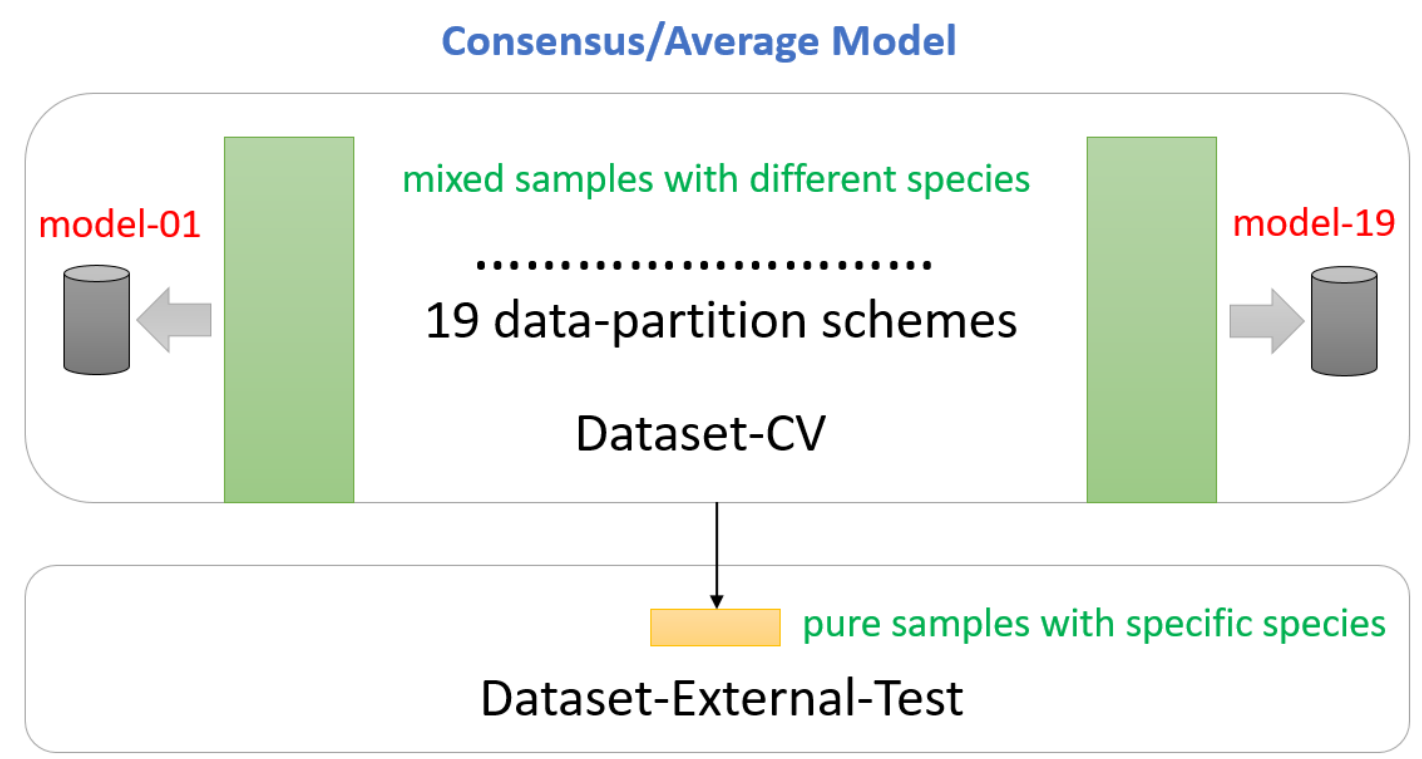

Requires sufficient and pure samples for each species in Dataset-External-Test

Figure S31. Protocol used to build the models on the basis of mixed samples containing different species, and evaluate the models on the pure samples from each species that are separated from the external test set. Yellow rectangle refers to Dataset-External-Test; Green rectangles refer to DatasetCV from 19 different data-splitting schemes that afford 19 individual models (model-01, model-02,..., model-18 and model-19). 


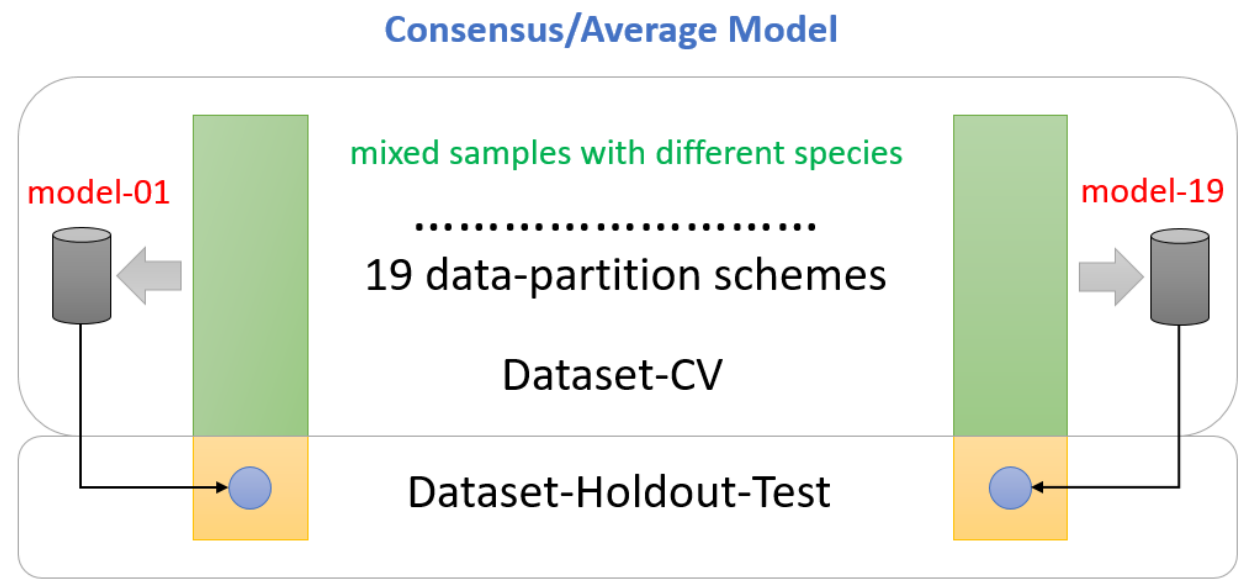

Figure S32. Protocol used to establish the models on the basis of mixed samples containing different species, and evaluate the models on the pure samples from each species that are extracted from the holdout test set. Blue circles refer to the samples for specific species. Green and yellow rectangles refer to Dataset-CV and Dataset-Holdout-Test respectively from 19 different data-splitting schemes that afford 19 individual models (model-01, model-02,.., model-18 and model-19). 


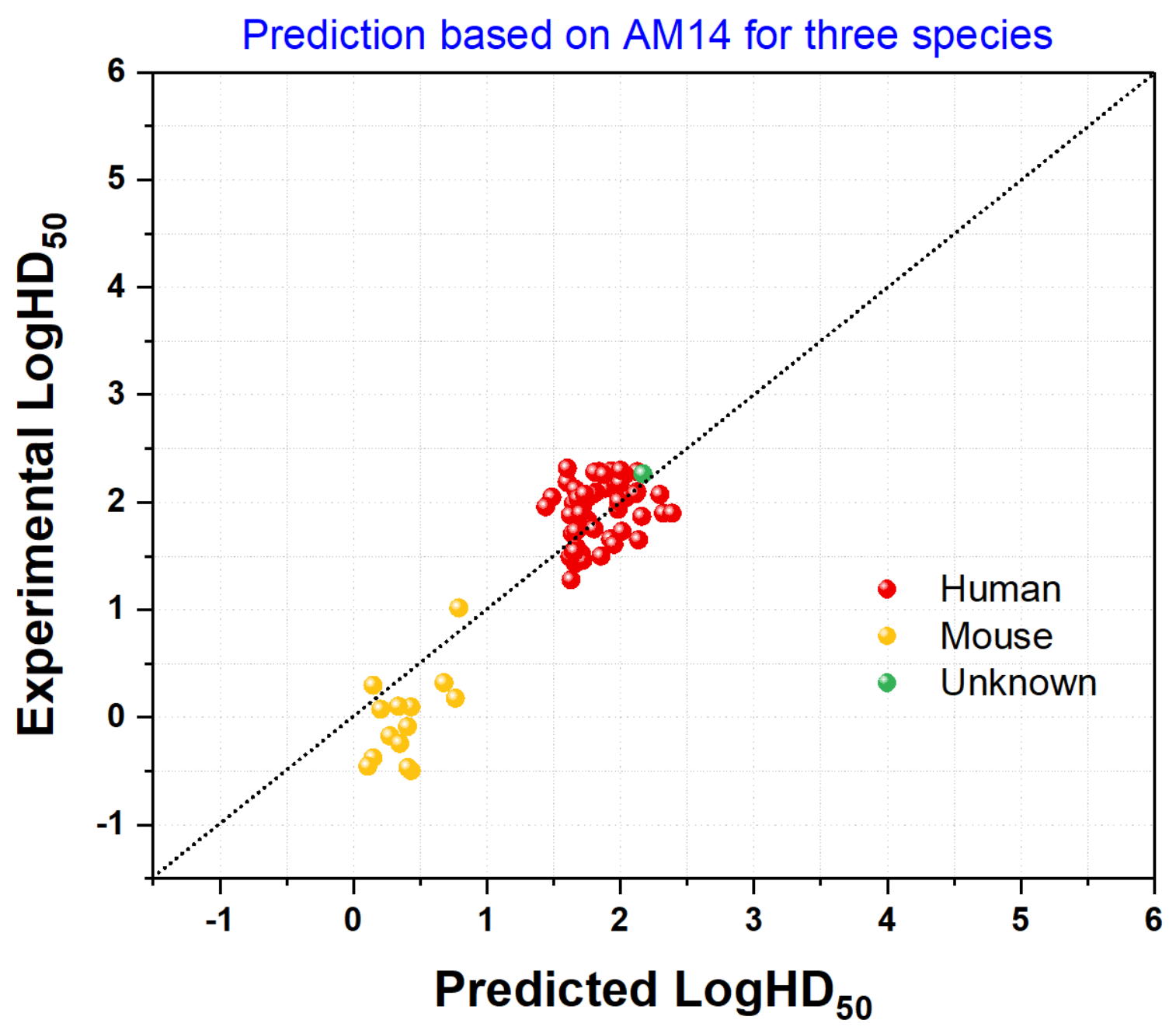

Figure S33. Scatter plot of predicted $\operatorname{LogHD} D_{50}$ vs. experimental $\log \mathrm{HD}_{50}$ for three species (Human, Mouse, and "Unknown") in the external test set. The predicted $\operatorname{LogHD}_{50}$ is obtained based on the best average model (AM14). 


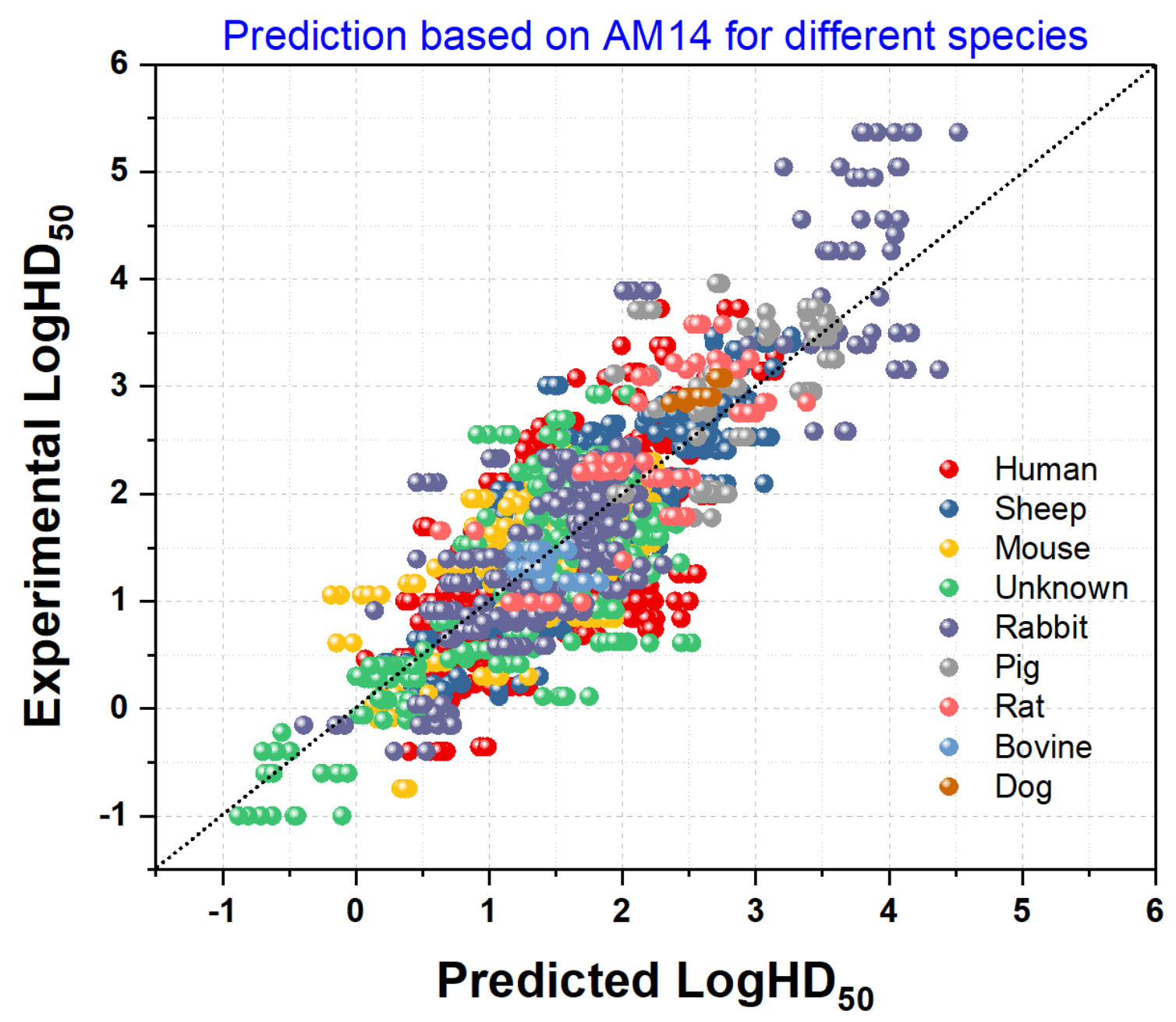

Figure S34. Scatter plot of predicted $\operatorname{LogHD}_{50}$ vs. experimental $\operatorname{LogHD} \mathrm{D}_{50}$ for different species. The predicted LogHD 50 is obtained based on the best average model (AM14). 


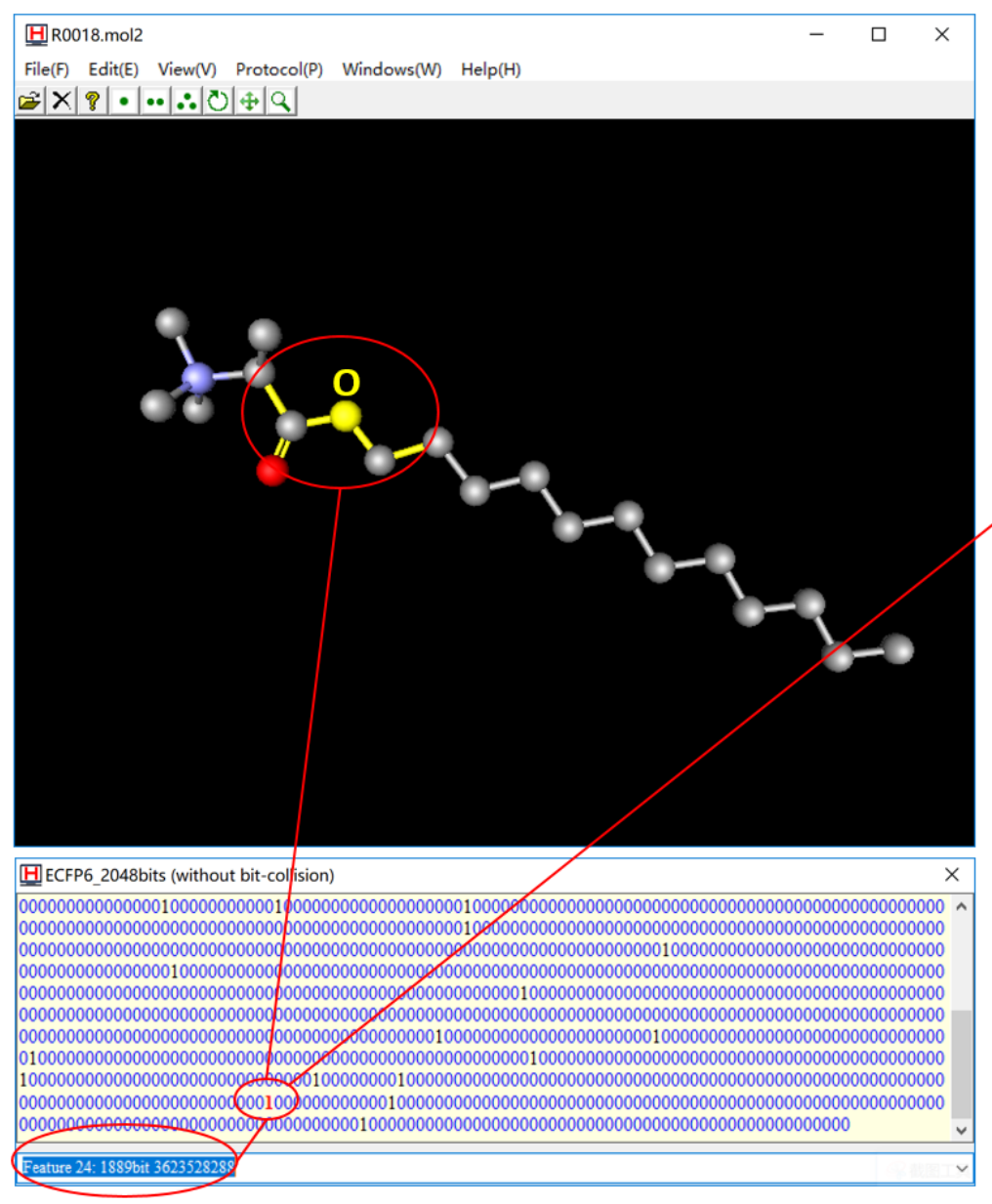

\begin{tabular}{|c|c|c|c|c|}
\hline $\begin{array}{l}\underline{\underline{E}} \mathrm{FI} \\
\text { File(F) }\end{array}$ & how & Click "so & $\begin{array}{l}\square \\
\mathrm{Fl}^{\prime \prime} \\
\text { ce Only }\end{array}$ & \\
\hline FI: 1889 & (1) 0 . & 49754 & & v \\
\hline FP Bit vs & eatus: & Importance (FI) & & $v$ \\
\hline Bit & $0 / 1$ & FI & FPD & $\Delta$ \\
\hline 1889 & 1 & 0.049754 & 0.000000 & \\
\hline 1890 & 0 & 0000025 & 0.000000 & \\
\hline 1891 & 0 & 0.000006 & 0.000000 & \\
\hline 1892 & & 0.000035 & 0.000000 & \\
\hline 1893 & 0 & 0.000012 & 0.000000 & \\
\hline 1894 & 0 & 0.002976 & 0.000000 & \\
\hline 1895 & 0 & 0.001550 & 0.000000 & \\
\hline 1896 & 0 & 0.000127 & 0.000000 & \\
\hline 1897 & 0 & 0.000091 & 0.000000 & \\
\hline 1898 & 0 & 0.000024 & 0.000000 & \\
\hline 1899 & 0 & 0.000015 & 0.000000 & \\
\hline 1900 & 0 & 0.000016 & 0.000000 & \\
\hline 1901 & 0 & 0.000001 & 0.000000 & \\
\hline 1902 & 1 & 0.000480 & 0.000000 & \\
\hline 1903 & 0 & 0.000005 & 0.000000 & \\
\hline 1904 & 0 & 0.000123 & 0.000000 & \\
\hline 1905 & 0 & 0.000260 & 0.000000 & \\
\hline 1906 & 0 & 0.004813 & 0.000000 & \\
\hline 1907 & 0 & 0.000054 & 0.000000 & \\
\hline 1908 & 0 & 0.000391 & 0.000000 & \\
\hline 1909 & 0 & 0.000008 & 0.000000 & \\
\hline 1910 & 0 & 0.000038 & 0.000000 & \\
\hline 1911 & 0 & 0.000015 & 0.000000 & \\
\hline 1912 & 0 & 0.000182 & 0.000000 & \\
\hline 1913 & 0 & 0.000481 & 0.000000 & \\
\hline 1914 & 0 & 0.000009 & 0.000000 & \\
\hline & & 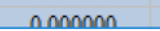 & 100000 & \\
\hline
\end{tabular}

Figure S35. Visualization of 3D structural feature for 1889-bit with the second largest feature importance $(\mathrm{FI}=0.049754)$ in the e-Hemolytic-Regression program. The central atom is oxygen and the bond diameter is 4 . It should be mentioned that this feature importance is derived from the random forest method. 


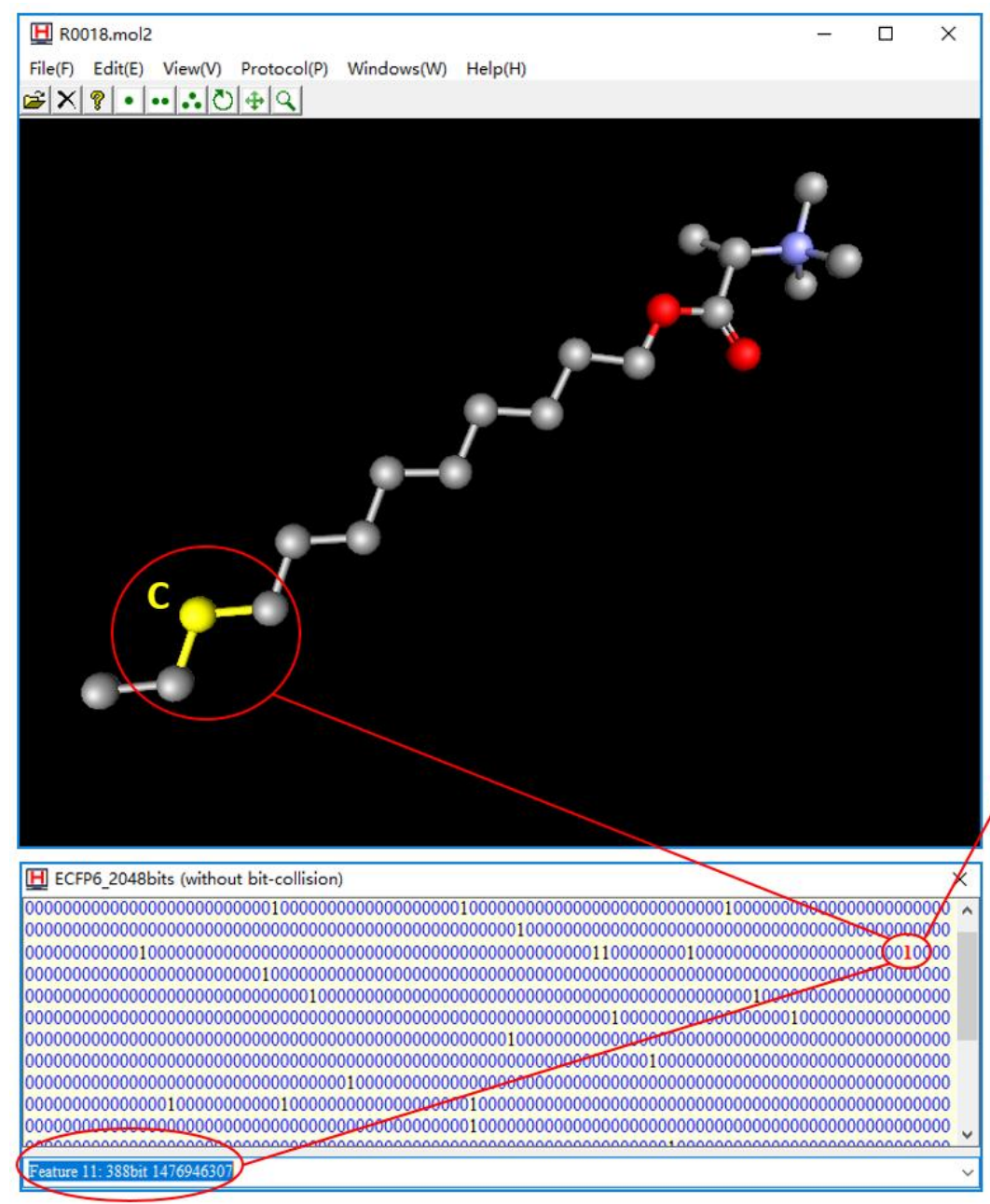

\begin{tabular}{|c|c|c|c|c|}
\hline EI & & - & $\square$ & \\
\hline File(F) & ol & Click "s & $\mathrm{Fl}^{\prime \prime}$ & \\
\hline & oov & eature Impo & ce Only & \\
\hline FI: $388 \mathrm{~b}$ & 0. & & & $v$ \\
\hline FP Bit vs & eatu & mportance (FI) & & $v$ \\
\hline Bit & $0 / 1$ & FI & FPD & $\Delta$ \\
\hline 388 & 1 & 0.029127 & 0.000000 & \\
\hline 389 & 0 & 0.000234 & 0.000000 & \\
\hline 390 & 0 & 0,000024 & 0.000000 & \\
\hline 391 & 0 & 0.000016 & 0.000000 & \\
\hline 392 & 0 & 0.000000 & 0.000000 & \\
\hline 393 & 0 & 0.001708 & 0.000000 & \\
\hline 394 & 0 & 0.000173 & 0.000000 & \\
\hline 395 & 6 & 0.000154 & 0.000000 & \\
\hline 396 & 0 & 0.000005 & 0.000000 & \\
\hline 397 & 0 & 0.000014 & 0.000000 & \\
\hline 398 & 0 & 0.000010 & 0.000000 & \\
\hline 390 & 0 & 0.000089 & 0.000000 & \\
\hline 400 & 0 & 0.000275 & 0.000000 & \\
\hline 401 & 0 & 0.000000 & 0.000000 & \\
\hline 402 & 0 & 0.000019 & 0.000000 & \\
\hline 403 & 0 & 0.000011 & 0.000000 & \\
\hline 404 & 0 & 0.000518 & 0.000000 & \\
\hline 405 & 0 & 0.002952 & 0.000000 & \\
\hline 406 & 0 & 0.000470 & 0.000000 & \\
\hline 407 & 0 & 0.000000 & 0.000000 & \\
\hline 408 & 0 & 0.001788 & 0.000000 & \\
\hline 409 & 0 & 0.000039 & 0.000000 & \\
\hline 410 & 0 & 0.002743 & 0.000000 & \\
\hline 411 & 0 & 0.000358 & 0.000000 & \\
\hline 412 & 0 & 0.000012 & 0.000000 & \\
\hline 413 & 0 & 0.000384 & 0.000000 & \\
\hline 414 & 0 & ח 0000023 & nomonos & \\
\hline
\end{tabular}

Figure S36. Visualization of 3D structural feature for 388-bit with the third largest feature importance $(\mathrm{FI}=0.029127)$ in the e-Hemolytic-Regression program. The central atom is carbon and the bond diameter is 2 . It should be noted that this feature importance is derived from the random forest method. 


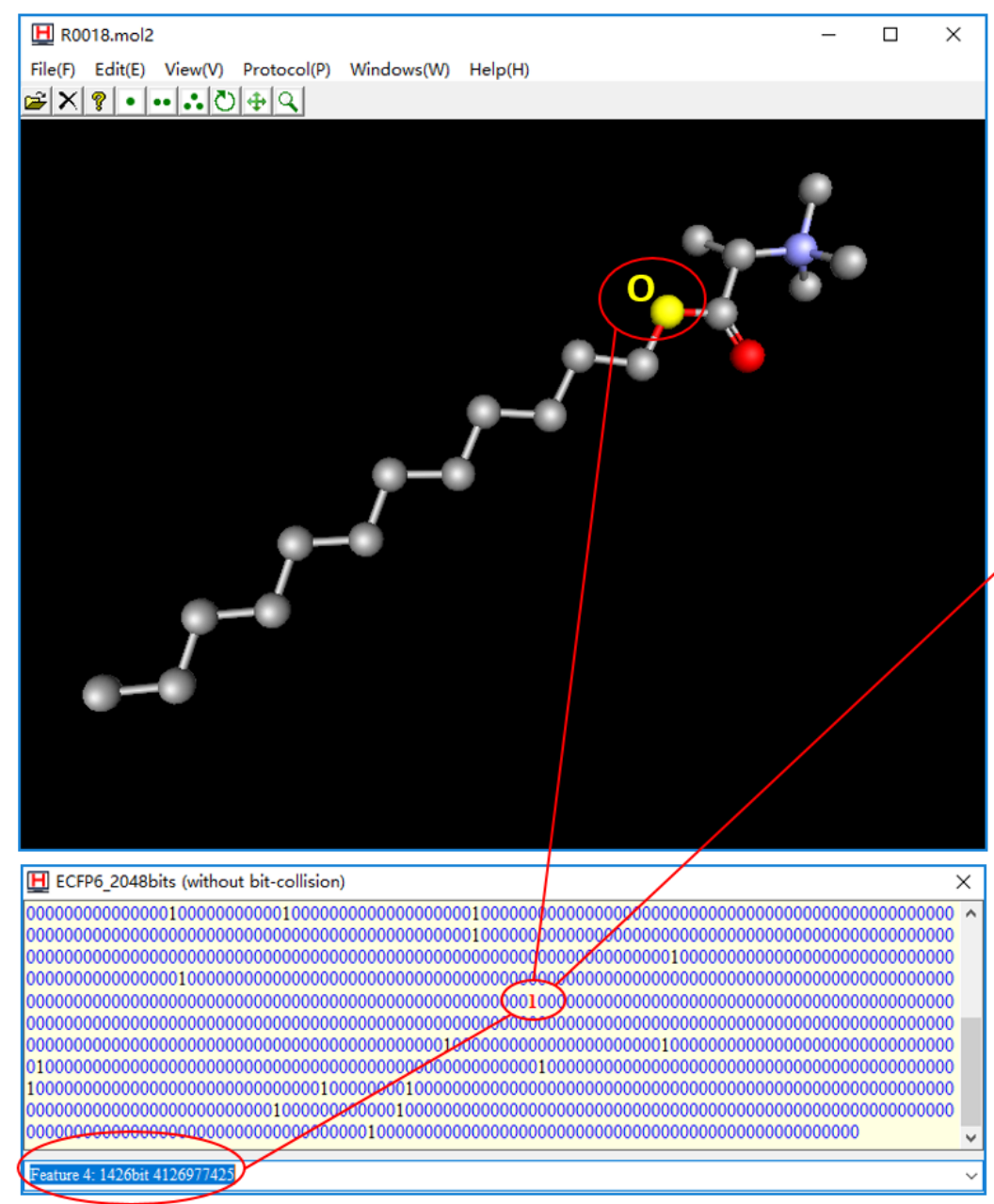

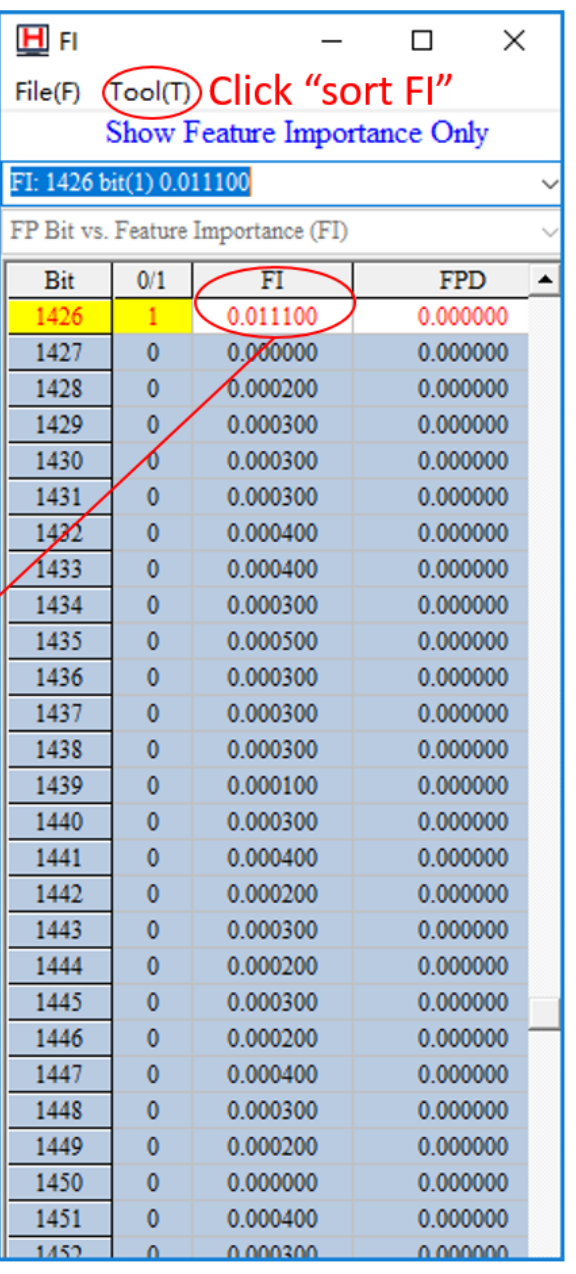

Figure S37. Visualization of 3D structural feature for 1426-bit with the second largest feature importance $(\mathrm{FI}=0.011100)$ in the e-Hemolytic-Regression program. The central atom is oxygen and the bond diameter is 0. It is worth noting that the feature importance refers to $\left|\Delta R^{2}\right|$ as defined in Figure 14, and is derived from the X-scrambling method. 


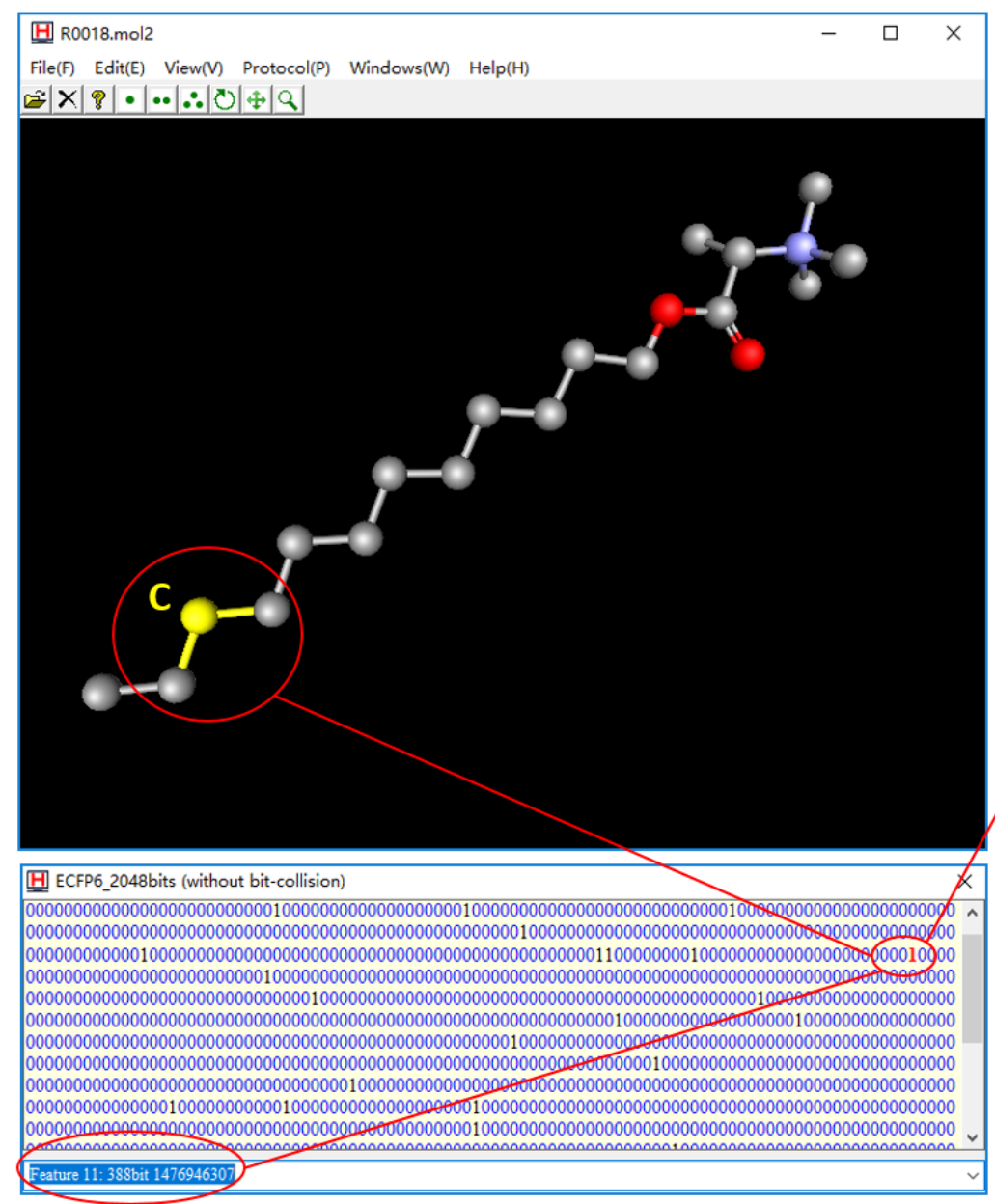

\begin{tabular}{|c|c|c|c|c|}
\hline \multicolumn{5}{|c|}{$\begin{array}{l}\text { File(F) Tool(T) Click "sort Fl" } \\
\text { Show Feature Importance Only }\end{array}$} \\
\hline \multicolumn{4}{|c|}{ FI: 388 bit(1) 0.009900} & $\checkmark$ \\
\hline \multicolumn{5}{|c|}{ FP Bit vs. Feature Importance (FI) } \\
\hline Bit & $0 / 1$ & FI & FPD & $\Delta$ \\
\hline 388 & 1 & 0.009900 & 0.000000 & \\
\hline 389 & 0 & 0.000200 & 0.000000 & \\
\hline 390 & 0 & 0,000300 & 0.000000 & \\
\hline 391 & 0 & 0.000300 & 0.000000 & \\
\hline 392 & 0 & 0.000400 & 0.000000 & \\
\hline 393 & 0 & 0.000400 & 0.000000 & \\
\hline 394 & 0 & 0.000400 & 0.000000 & \\
\hline 395 & 6 & 0.000300 & 0.000000 & \\
\hline 396 & 0 & 0.000300 & 0.000000 & \\
\hline 397 & 0 & 0.000300 & 0.000000 & \\
\hline 398 & 0 & 0.000400 & 0.000000 & \\
\hline $39 \%$ & 0 & 0.000000 & 0.000000 & \\
\hline 400 & 0 & 0.000100 & 0.000000 & \\
\hline 401 & 0 & 0.000400 & 0.000000 & \\
\hline 402 & 0 & 0.000300 & 0.000000 & \\
\hline 403 & 0 & 0.000300 & 0.000000 & \\
\hline 404 & 0 & 0.000400 & 0.000000 & \\
\hline 405 & 0 & 0.000600 & 0.000000 & \\
\hline 406 & 0 & 0.000300 & 0.000000 & \\
\hline 407 & 0 & 0.000400 & 0.000000 & \\
\hline 408 & 0 & 0.000300 & 0.000000 & \\
\hline 409 & 0 & 0.000300 & 0.000000 & \\
\hline 410 & 0 & 0.000200 & 0.000000 & \\
\hline 411 & 0 & 0.000500 & 0.000000 & \\
\hline 412 & 0 & 0.000300 & 0.000000 & \\
\hline 413 & 0 & 0.000300 & 0.000000 & \\
\hline 411 & 0 & 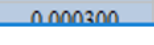 & nononon & \\
\hline
\end{tabular}

Figure S38. Visualization of 3D structural feature for 388-bit with the third largest feature importance $(\mathrm{FI}=0.009900)$ in the e-Hemolytic-Regression program. The central atom is carbon and the bond diameter is 2 . It should be noted that the feature importance refers to $\left|\Delta R^{2}\right|$ as defined in Figure 14, and is derived from the $X$ scrambling method. 


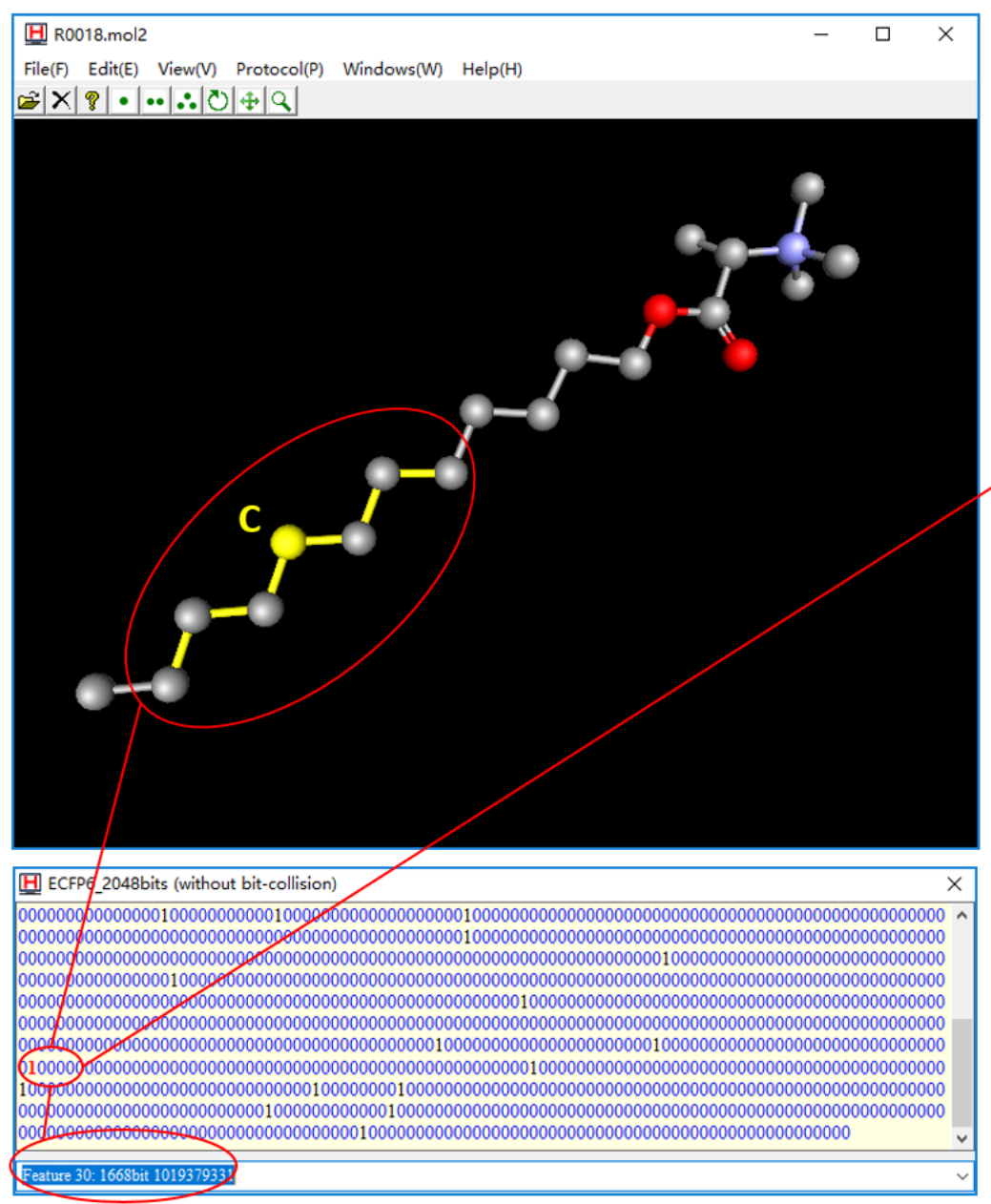

\begin{tabular}{|c|c|c|c|c|}
\hline $\begin{array}{l}\text { 트 FI } \\
\text { File(F) }\end{array}$ & ool( & Click "so & $\begin{array}{ll}\square & \times \\
F^{\prime \prime} & \end{array}$ & \\
\hline & how & eature Impor & e Only & \\
\hline FI: 1668 & (1) 0. & 9400 & & $\checkmark$ \\
\hline FP Bit vs & eatur & Importance (FI) & & $\checkmark$ \\
\hline Bit & $0 / 1$ & FI & FPD & $\Delta$ \\
\hline 1668 & 1 & 0.009400 & 0.000000 & \\
\hline 1669 & 0 & Q.000200 & 0.000000 & \\
\hline 1670 & & 0.000300 & 0.000000 & \\
\hline 1671 & 0 & 0.000300 & 0.000000 & \\
\hline 1672 & 0 & 0.000000 & 0.000000 & \\
\hline 1673 & 0 & 0.000200 & 0.000000 & \\
\hline 1674 & 0 & 0.000200 & 0.000000 & \\
\hline 1675 & 0 & 0.000000 & 0.000000 & \\
\hline 1676 & 0 & 0.000300 & 0.000000 & \\
\hline 1677 & 0 & 0.000200 & 0.000000 & \\
\hline 1678 & 0 & 0.000400 & 0.000000 & \\
\hline 1679 & 0 & 0.000400 & 0.000000 & \\
\hline 1680 & 0 & 0.000400 & 0.000000 & \\
\hline 1681 & 0 & 0.000300 & 0.000000 & \\
\hline 1682 & 0 & 0.000300 & 0.000000 & \\
\hline 1683 & 0 & 0.000200 & 0.000000 & \\
\hline 1684 & 0 & 0.000100 & 0.000000 & \\
\hline 1685 & 0 & 0.000200 & 0.000000 & \\
\hline 1686 & 0 & 0.000300 & 0.000000 & \\
\hline 1687 & 0 & 0.000100 & 0.000000 & \\
\hline 1688 & 0 & 0.000400 & 0.000000 & \\
\hline 1689 & 0 & 0.000600 & 0.000000 & \\
\hline 1690 & 0 & 0.000300 & 0.000000 & \\
\hline 1691 & 0 & 0.000300 & 0.000000 & \\
\hline 1692 & 0 & 0.000300 & 0.000000 & \\
\hline 1693 & 0 & 0.000400 & 0.000000 & \\
\hline 1604 & 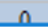 & 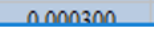 & 年 & \\
\hline
\end{tabular}

Figure S39. Visualization of 3D structural feature for 1668-bit with the fourth largest feature importance $(\mathrm{FI}=0.009400)$ in the e-Hemolytic-Regression program. The central atom is carbon and the bond diameter is 6 . It is worth mentioning that the feature importance refers to $\left|\Delta \mathrm{R}^{2}\right|$ as defined in Figure 14, and is derived from the Xscrambling method. This structural feature is very similar to that of 1613-bit, however, 1613-bit contains the terminal aliphatic carbon that is not existed in 1668-bit. 


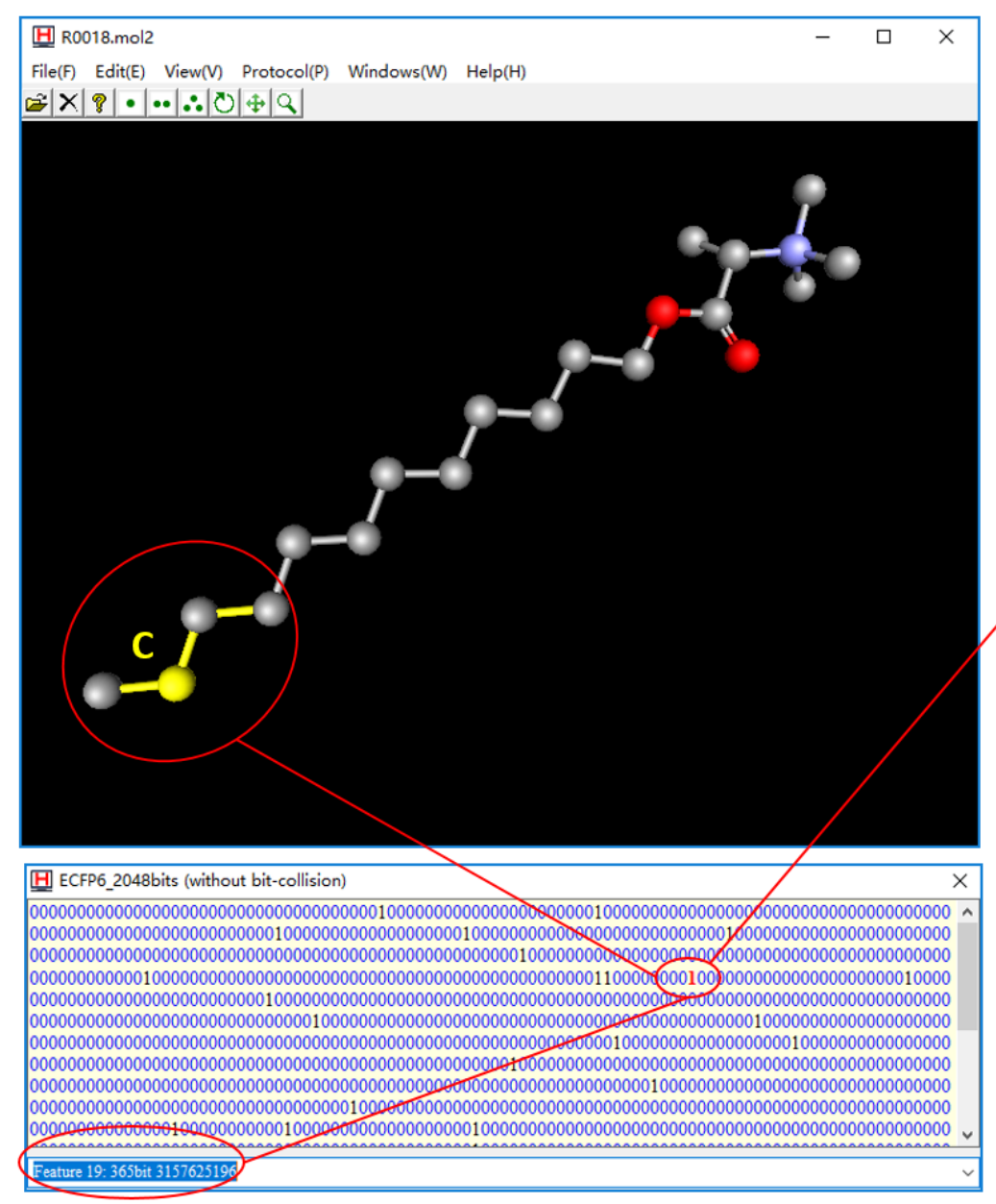

\begin{tabular}{|c|c|c|c|c|}
\hline $\begin{array}{l}\text { 표 Fl } \\
\text { File(F) }\end{array}$ & $\frac{001(7)}{6}$ & Click "so & $\begin{array}{l}\square \\
\mathrm{Fl}^{\prime \prime} \\
\text { ce Only }\end{array}$ & \\
\hline FI: $365 t$ & 0.0 & 7500 & & $v$ \\
\hline FP Bit $v$ & ieatur & Importance (FI) & & $v$ \\
\hline Bit & $0 / 1$ & FI & FPD & $\Delta$ \\
\hline 365 & 1 & 0.007500 & 0.000000 & \\
\hline 366 & 0 & 0.062000 & 0.000000 & \\
\hline 367 & 0 & 0.000300 & 0.000000 & \\
\hline 368 & 0 & 0.000200 & 0.000000 & \\
\hline 369 & 0 & 0.000300 & 0.000000 & \\
\hline 370 & 0 & 0.000000 & 0.000000 & \\
\hline 371 & 0 & 0.000200 & 0.000000 & \\
\hline 372 & 0 & 0.000300 & 0.000000 & \\
\hline 373 & 0 & 0.000300 & 0.000000 & \\
\hline 374 & 0 & 0.000100 & 0.000000 & \\
\hline 375 & 0 & 0.000300 & 0.000000 & \\
\hline 376 & 0 & 0.000300 & 0.000000 & \\
\hline 377 & 0 & 0.001000 & 0.000000 & \\
\hline 378 & 0 & 0.000300 & 0.000000 & \\
\hline 379 & 0 & 0.000300 & 0.000000 & \\
\hline 380 & 0 & 0.000400 & 0.000000 & \\
\hline 381 & 0 & 0.000300 & 0.000000 & \\
\hline 382 & 0 & 0.000300 & 0.000000 & \\
\hline 383 & 0 & 0.000800 & 0.000000 & \\
\hline 384 & 0 & 0.000400 & 0.000000 & \\
\hline 385 & 0 & 0.000400 & 0.000000 & \\
\hline 386 & 0 & 0.000300 & 0.000000 & \\
\hline 387 & 0 & 0.000400 & 0.000000 & \\
\hline 388 & 1 & 0.009900 & 0.000000 & \\
\hline 389 & 0 & 0.000200 & 0.000000 & \\
\hline 390 & 0 & 0.000300 & 0.000000 & \\
\hline 301 & 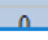 & 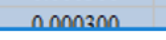 & ח ח ח & \\
\hline
\end{tabular}

Figure S40. Visualization of 3D structural feature for 365-bit with the fifth largest feature importance $(\mathrm{FI}=0.007500)$ in the e-Hemolytic-Regression program. The central atom is carbon and the bond diameter is 4 . It is noteworthy that the feature importance refers to $\left|\Delta R^{2}\right|$ as defined in Figure 14, and is derived from the $X$ scrambling method. 


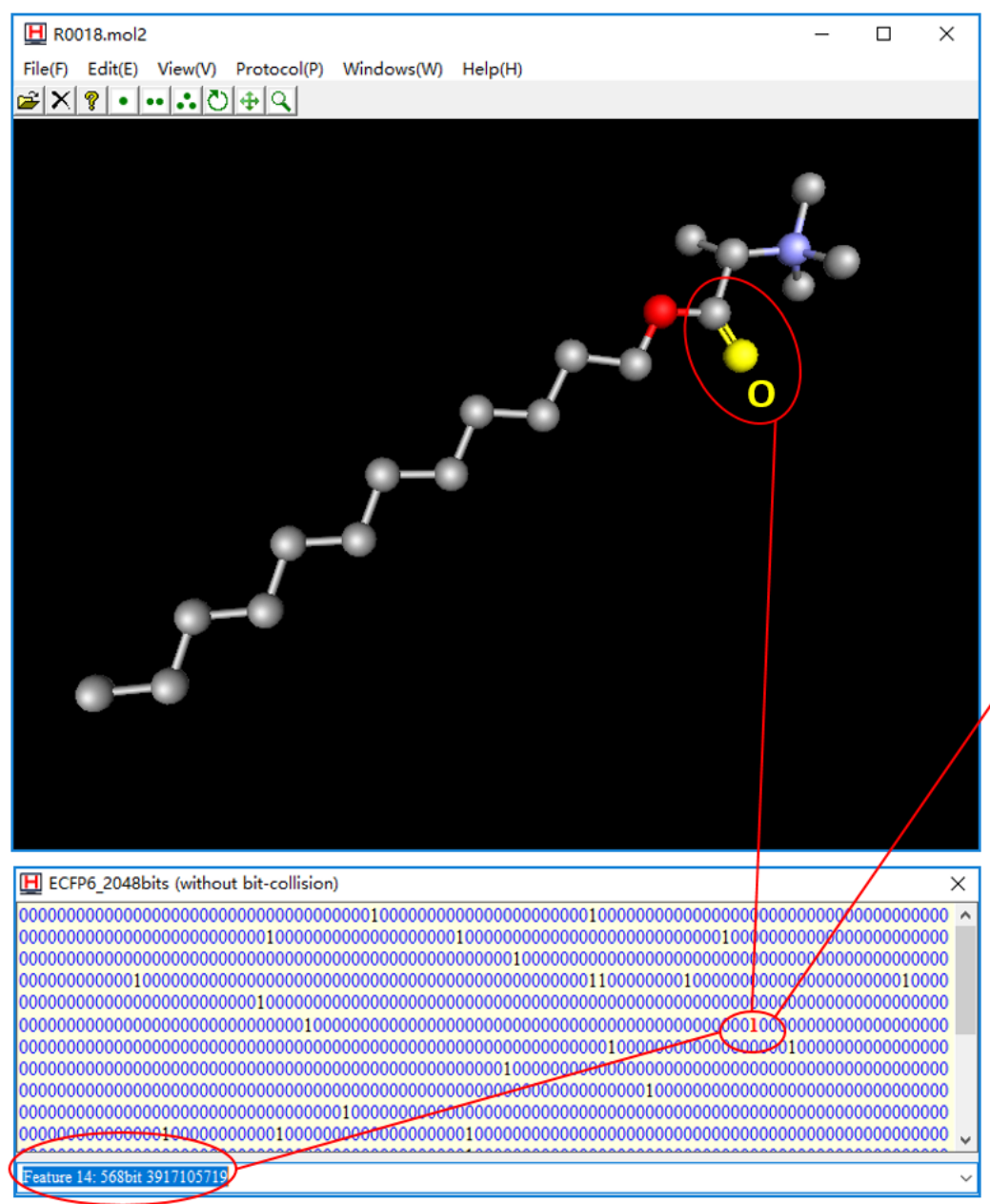

\begin{tabular}{|c|c|c|c|c|}
\hline $\begin{array}{l}\text { 프 FI } \\
\text { File(F) }\end{array}$ & $\frac{\mathrm{oll}}{\mathrm{cow}}$ & Click "sc & $\begin{array}{l}\square \\
\mathrm{Fl}^{\prime \prime} \\
\text { enly }\end{array}$ & \\
\hline FI: $568 \mathrm{bi}$ & 0. & 700 & & $\checkmark$ \\
\hline FP Bit vs & eatu & Importance (FI) & & $v$ \\
\hline Bit & $0 / 1$ & FI & FPD & $\Delta$ \\
\hline 568 & 1 & 0.005700 & 0.000000 & \\
\hline 569 & 0 & 0.080100 & 0.000000 & \\
\hline 570 & 0 & 9.000200 & 0.000000 & \\
\hline 571 & 0 & 0.000300 & 0.000000 & \\
\hline 572 & 0 & 0.000400 & 0.000000 & \\
\hline 573 & g) & 0.000000 & 0.000000 & \\
\hline 574 & 0 & 0.000400 & 0.000000 & \\
\hline 575 & 0 & 0.000100 & 0.000000 & \\
\hline 576 & 0 & 0.000000 & 0.000000 & \\
\hline $57 h$ & 0 & 0.000200 & 0.000000 & \\
\hline 578 & 0 & 0.000300 & 0.000000 & \\
\hline 579 & 0 & 0.000300 & 0.000000 & \\
\hline 580 & 0 & 0.000400 & 0.000000 & \\
\hline 581 & 0 & 0.000300 & 0.000000 & \\
\hline 582 & 0 & 0.000400 & 0.000000 & \\
\hline 583 & 0 & 0.000400 & 0.000000 & \\
\hline 584 & 0 & 0.000300 & 0.000000 & \\
\hline 585 & 0 & 0.000300 & 0.000000 & \\
\hline 586 & 0 & 0.000200 & 0.000000 & \\
\hline 587 & 0 & 0.000500 & 0.000000 & \\
\hline 588 & 0 & 0.000300 & 0.000000 & \\
\hline 589 & 0 & 0.000400 & 0.000000 & \\
\hline 590 & 0 & 0.000100 & 0.000000 & \\
\hline 591 & 0 & 0.000300 & 0.000000 & \\
\hline 592 & 0 & 0.000300 & 0.000000 & \\
\hline 593 & 0 & 0.000300 & 0.000000 & \\
\hline 50 & n & nomonom & nomonom & \\
\hline
\end{tabular}

Figure S41. Visualization of 3D structural feature for 568-bit with the sixth largest feature importance $(\mathrm{FI}=0.005700)$ in the e-Hemolytic-Regression program. The central atom is oxygen in the carbonyl group and the bond diameter is 2 . It should be noted that the feature importance refers to $\left|\Delta R^{2}\right|$ as defined in Figure 14, and is derived from the $\mathrm{X}$-scrambling method. 
Table S1. Average similarity and standard deviation of all the similarities in each similarity matrix for each data-partition scheme

\begin{tabular}{|c|c|c|}
\hline $\begin{array}{c}\text { Data-partition } \\
\text { scheme }\end{array}$ & $\begin{array}{c}\text { Average similarity of all the } \\
\text { similarities in similarity matrix }\end{array}$ & $\begin{array}{c}\text { Standard deviation of all the } \\
\text { similarities in similarity matrix }\end{array}$ \\
\hline 1 & 0.11 & 0.12 \\
\hline 2 & 0.12 & 0.12 \\
\hline 3 & 0.11 & 0.12 \\
\hline 4 & 0.12 & 0.13 \\
\hline 5 & 0.12 & 0.13 \\
\hline 6 & 0.12 & 0.13 \\
\hline 7 & 0.11 & 0.12 \\
\hline 8 & 0.12 & 0.12 \\
\hline 9 & 0.12 & 0.13 \\
\hline 10 & 0.11 & 0.12 \\
\hline 11 & 0.12 & 0.13 \\
\hline 12 & 0.11 & 0.12 \\
\hline 13 & 0.12 & 0.12 \\
\hline 14 & 0.12 & 0.13 \\
\hline 15 & 0.12 & 0.13 \\
\hline 16 & 0.12 & 0.12 \\
\hline 17 & 0.12 & 0.13 \\
\hline 18 & 0.12 & 0.12 \\
\hline 19 & 0.11 & 0.12 \\
\hline
\end{tabular}


Table S2. The parameters for each machine learning method that needs to be fine-tuned in the five-fold cross-validation

\begin{tabular}{|c|l|}
\hline Machine-learning methods & Parameters \\
\hline KNN & $\begin{array}{l}\text { Number of nearest neighbors }(\mathrm{K}): 1,3,5,7,9,11,13, \text { and } 15 \\
\text { Weighting schemes: uniform weight or distance-dependent weight }\end{array}$ \\
\hline SVM & $\begin{array}{l}\text { Kernel: RBF } \\
\text { Penalty parameter C: } 1,000,5,000,10,000,50,000, \text { and } 100,000 \\
\text { Kernel parameter gamma: } 0.0001,0.0005,0.001,0.005,0.01, \text { and } 0.1\end{array}$ \\
\hline RF & Number of decision trees: $10,50,100,200,300,400,500,600,700,800,900$, and 1,000 \\
\hline GBM & $\begin{array}{l}\text { Number of decision trees: } 10,50,100,200,300,400,500,600,700,800,900, \text { and } 1,000 \\
\text { Learning rate: } 0.1,0.2,0.3,0.4,0.5,0.6,0.7,0.8, \text { and } 0.9\end{array}$ \\
\hline
\end{tabular}


Table S3. 1216 individual regression models derived from Dataset-CV (Dataset-Train and Dataset-Internal-Validation) and evaluated on DatasetHoldout-Test.

\begin{tabular}{|c|c|c|c|c|c|c|c|c|c|c|}
\hline Model & FS & EXP & FP & Method & $\begin{array}{c}\mathbf{R}^{2} \\
\text { (holdout } \\
\text { test) }\end{array}$ & $\begin{array}{c}\text { MSE } \\
\text { (holdout } \\
\text { test) }\end{array}$ & $\begin{array}{c}\text { MAE } \\
\text { (holdout } \\
\text { test) }\end{array}$ & $\begin{array}{c}\mathbf{R}^{2} \\
(\mathbf{C V})\end{array}$ & $\Delta \mathbf{R}^{2}$ & $\begin{array}{l}\text { Parameters from } \\
\text { CV* }\end{array}$ \\
\hline M0001 & full & exp01 & 1024bit-ECFP4 & KNN & 0.661 & 0.260 & 0.372 & 0.599 & 0.062 & 5 distance \\
\hline M0002 & full & exp02 & 1024bit-ECFP4 & KNN & 0.630 & 0.274 & 0.388 & 0.633 & 0.003 & 5 distance \\
\hline M0003 & full & $\exp 03$ & 1024bit-ECFP4 & KNN & 0.599 & 0.303 & 0.388 & 0.614 & 0.015 & 3 distance \\
\hline M0004 & full & $\exp 04$ & 1024bit-ECFP4 & KNN & 0.534 & 0.367 & 0.441 & 0.625 & 0.091 & 3 distance \\
\hline M0005 & full & exp05 & 1024bit-ECFP4 & KNN & 0.639 & 0.256 & 0.386 & 0.630 & 0.009 & 3 uniform \\
\hline M0006 & full & $\exp 06$ & 1024bit-ECFP4 & $\mathrm{KNN}$ & 0.638 & 0.261 & 0.395 & 0.604 & 0.034 & 5 distance \\
\hline M0007 & full & $\exp 07$ & 1024bit-ECFP4 & KNN & 0.572 & 0.329 & 0.407 & 0.635 & 0.064 & 3 distance \\
\hline M0008 & full & $\exp 08$ & 1024bit-ECFP4 & KNN & 0.608 & 0.294 & 0.398 & 0.635 & 0.027 & 3 distance \\
\hline M0009 & full & exp09 & 1024bit-ECFP4 & KNN & 0.534 & 0.348 & 0.403 & 0.592 & 0.058 & 3 distance \\
\hline M0010 & full & $\exp 10$ & 1024bit-ECFP4 & $\mathrm{KNN}$ & 0.650 & 0.255 & 0.381 & 0.612 & 0.038 & 3 distance \\
\hline M0011 & full & exp11 & 1024bit-ECFP4 & KNN & 0.597 & 0.309 & 0.402 & 0.567 & 0.030 & 5 distance \\
\hline M0012 & full & exp12 & 1024bit-ECFP4 & KNN & 0.601 & 0.296 & 0.413 & 0.616 & 0.015 & 3 distance \\
\hline M0013 & full & $\exp 13$ & 1024bit-ECFP4 & KNN & 0.660 & 0.243 & 0.375 & 0.613 & 0.047 & 5 distance \\
\hline M0014 & full & exp14 & 1024bit-ECFP4 & KNN & 0.564 & 0.345 & 0.437 & 0.638 & 0.074 & 3 distance \\
\hline M0015 & full & $\exp 15$ & 1024bit-ECFP4 & KNN & 0.644 & 0.288 & 0.379 & 0.588 & 0.056 & 3 distance \\
\hline M0016 & full & $\exp 16$ & 1024bit-ECFP4 & $\mathrm{KNN}$ & 0.619 & 0.276 & 0.397 & 0.620 & 0.001 & 5 distance \\
\hline M0017 & full & exp17 & 1024bit-ECFP4 & KNN & 0.588 & 0.309 & 0.409 & 0.617 & 0.029 & 5 distance \\
\hline M0018 & full & $\exp 18$ & 1024bit-ECFP4 & KNN & 0.619 & 0.276 & 0.406 & 0.608 & 0.011 & 5 distance \\
\hline M0019 & full & exp19 & 1024bit-ECFP4 & KNN & 0.576 & 0.307 & 0.408 & 0.641 & 0.065 & 7 distance \\
\hline M0020 & full & $\exp 01$ & 1024bit-ECFP4 & SVM & 0.696 & 0.212 & 0.352 & 0.641 & 0.055 & 100.01 \\
\hline M0021 & full & $\exp 02$ & 1024bit-ECFP4 & SVM & 0.666 & 0.246 & 0.366 & 0.656 & 0.010 & 100.01 \\
\hline M0022 & full & exp03 & 1024bit-ECFP4 & SVM & 0.648 & 0.243 & 0.366 & 0.655 & 0.007 & 50.01 \\
\hline M0023 & full & $\exp 04$ & 1024bit-ECFP4 & SVM & 0.621 & 0.255 & 0.384 & 0.651 & 0.030 & 1000.01 \\
\hline M0024 & full & $\exp 05$ & 1024bit-ECFP4 & SVM & 0.665 & 0.227 & 0.364 & 0.656 & 0.009 & 50.05 \\
\hline M0025 & full & $\exp 06$ & 1024bit-ECFP4 & SVM & 0.622 & 0.269 & 0.386 & 0.643 & 0.021 & 50.01 \\
\hline M0026 & full & exp07 & 1024bit-ECFP4 & SVM & 0.576 & 0.295 & 0.406 & 0.672 & 0.096 & 100.01 \\
\hline M0027 & full & $\exp 08$ & 1024bit-ECFP4 & SVM & 0.623 & 0.260 & 0.387 & 0.681 & 0.058 & 500.01 \\
\hline M0028 & full & exp09 & 1024bit-ECFP4 & SVM & 0.592 & 0.278 & 0.396 & 0.630 & 0.038 & 50.01 \\
\hline M0029 & full & $\exp 10$ & 1024bit-ECFP4 & SVM & 0.627 & 0.261 & 0.390 & 0.641 & 0.014 & 50.005 \\
\hline M0030 & full & $\exp 11$ & 1024bit-ECFP4 & SVM & 0.663 & 0.231 & 0.364 & 0.614 & 0.049 & 50.005 \\
\hline M0031 & full & exp12 & 1024bit-ECFP4 & SVM & 0.618 & 0.260 & 0.377 & 0.639 & 0.021 & 100.01 \\
\hline M0032 & full & $\exp 13$ & 1024bit-ECFP4 & SVM & 0.693 & 0.224 & 0.362 & 0.623 & 0.070 & 50.01 \\
\hline M0033 & full & exp14 & 1024bit-ECFP4 & SVM & 0.602 & 0.284 & 0.406 & 0.676 & 0.074 & 100.01 \\
\hline M0034 & full & $\exp 15$ & 1024bit-ECFP4 & SVM & 0.702 & 0.215 & 0.343 & 0.627 & 0.075 & 100.01 \\
\hline M0035 & full & exp16 & 1024bit-ECFP4 & SVM & 0.632 & 0.248 & 0.383 & 0.641 & 0.009 & 100.01 \\
\hline
\end{tabular}


Supporting Information (Texts S1-S9, Figures S1-S41 and Tables S1-S12)

\begin{tabular}{|c|c|c|c|c|c|c|c|c|c|c|}
\hline M0036 & full & exp17 & 1024bit-ECFP4 & SVM & 0.588 & 0.297 & 0.406 & 0.652 & 0.064 & 100.01 \\
\hline M0037 & full & exp18 & 1024bit-ECFP4 & SVM & 0.637 & 0.243 & 0.387 & 0.628 & 0.009 & 50.05 \\
\hline M0038 & full & exp19 & 1024bit-ECFP4 & SVM & 0.651 & 0.242 & 0.372 & 0.659 & 0.008 & 50.05 \\
\hline M0039 & full & exp01 & 1024bit-ECFP4 & GBM & 0.659 & 0.237 & 0.372 & 0.591 & 0.068 & 1000.3 \\
\hline M0040 & full & exp02 & 1024bit-ECFP4 & GBM & 0.676 & 0.238 & 0.367 & 0.607 & 0.069 & 1000.2 \\
\hline M0041 & full & exp03 & 1024bit-ECFP4 & GBM & 0.618 & 0.262 & 0.378 & 0.640 & 0.022 & 4000.1 \\
\hline M0042 & full & exp04 & 1024bit-ECFP4 & GBM & 0.568 & 0.287 & 0.409 & 0.618 & 0.050 & 500.3 \\
\hline M0043 & full & exp05 & 1024bit-ECFP4 & GBM & 0.650 & 0.234 & 0.374 & 0.626 & 0.024 & 500.2 \\
\hline M0044 & full & exp06 & 1024bit-ECFP4 & GBM & 0.596 & 0.285 & 0.391 & 0.610 & 0.014 & 3000.1 \\
\hline M0045 & full & exp07 & 1024bit-ECFP4 & GBM & 0.579 & 0.289 & 0.414 & 0.623 & 0.044 & 3000.1 \\
\hline M0046 & full & exp08 & 1024bit-ECFP4 & GBM & 0.576 & 0.290 & 0.412 & 0.662 & 0.086 & 3000.1 \\
\hline M0047 & full & exp09 & 1024bit-ECFP4 & GBM & 0.529 & 0.318 & 0.419 & 0.599 & 0.070 & 1000.1 \\
\hline M0048 & full & exp10 & 1024bit-ECFP4 & GBM & 0.657 & 0.240 & 0.374 & 0.601 & 0.056 & 3000.1 \\
\hline M0049 & full & exp11 & 1024bit-ECFP4 & GBM & 0.635 & 0.254 & 0.374 & 0.587 & 0.048 & 3000.1 \\
\hline M0050 & full & exp12 & 1024bit-ECFP4 & GBM & 0.546 & 0.301 & 0.412 & 0.612 & 0.066 & 1000.2 \\
\hline M0051 & full & exp13 & 1024bit-ECFP4 & GBM & 0.686 & 0.224 & 0.368 & 0.613 & 0.073 & 2000.1 \\
\hline M0052 & full & exp14 & 1024bit-ECFP4 & GBM & 0.604 & 0.280 & 0.405 & 0.633 & 0.029 & 2000.2 \\
\hline M0053 & full & exp15 & 1024bit-ECFP4 & GBM & 0.657 & 0.237 & 0.372 & 0.608 & 0.049 & 500.3 \\
\hline M0054 & full & exp16 & 1024bit-ECFP4 & GBM & 0.545 & 0.302 & 0.415 & 0.630 & 0.085 & 1000.1 \\
\hline M0055 & full & exp17 & 1024bit-ECFP4 & GBM & 0.621 & 0.272 & 0.387 & 0.612 & 0.009 & 6000.1 \\
\hline M0056 & full & exp18 & 1024bit-ECFP4 & GBM & 0.652 & 0.233 & 0.382 & 0.599 & 0.053 & 2000.1 \\
\hline M0057 & full & exp19 & 1024bit-ECFP4 & GBM & 0.636 & 0.249 & 0.373 & 0.622 & 0.014 & 1000.3 \\
\hline M0058 & full & exp01 & 1024bit-ECFP4 & $\mathrm{RF}$ & 0.692 & 0.214 & 0.344 & 0.621 & 0.071 & 500 \\
\hline M0059 & full & $\operatorname{exp02}$ & 1024bit-ECFP4 & $\mathrm{RF}$ & 0.698 & 0.224 & 0.355 & 0.617 & 0.081 & 400 \\
\hline M0060 & full & exp03 & 1024bit-ECFP4 & $\mathrm{RF}$ & 0.638 & 0.247 & 0.369 & 0.653 & 0.015 & 800 \\
\hline M0061 & full & exp04 & 1024bit-ECFP4 & $\mathrm{RF}$ & 0.616 & 0.257 & 0.379 & 0.634 & 0.018 & 200 \\
\hline M0062 & full & exp05 & 1024bit-ECFP4 & $\mathrm{RF}$ & 0.656 & 0.229 & 0.358 & 0.657 & 0.001 & 500 \\
\hline M0063 & full & exp06 & 1024bit-ECFP4 & $\mathrm{RF}$ & 0.616 & 0.271 & 0.390 & 0.648 & 0.032 & 200 \\
\hline M0064 & full & exp07 & 1024bit-ECFP4 & $\mathrm{RF}$ & 0.579 & 0.290 & 0.403 & 0.649 & 0.070 & 200 \\
\hline M0065 & full & exp08 & 1024bit-ECFP4 & $\mathrm{RF}$ & 0.621 & 0.264 & 0.388 & 0.668 & 0.047 & 1000 \\
\hline M0066 & full & exp09 & 1024bit-ECFP4 & $\mathrm{RF}$ & 0.523 & 0.322 & 0.412 & 0.615 & 0.092 & 400 \\
\hline M0067 & full & exp10 & 1024bit-ECFP4 & $\mathrm{RF}$ & 0.627 & 0.261 & 0.386 & 0.617 & 0.010 & 500 \\
\hline M0068 & full & exp11 & 1024bit-ECFP4 & $\mathrm{RF}$ & 0.623 & 0.260 & 0.371 & 0.596 & 0.027 & 200 \\
\hline M0069 & full & exp12 & 1024bit-ECFP4 & $\mathrm{RF}$ & 0.583 & 0.276 & 0.395 & 0.633 & 0.050 & 300 \\
\hline M0070 & full & exp13 & 1024bit-ECFP4 & $\mathrm{RF}$ & 0.730 & 0.199 & 0.347 & 0.626 & 0.104 & 900 \\
\hline M0071 & full & exp14 & 1024bit-ECFP4 & $\mathrm{RF}$ & 0.592 & 0.287 & 0.404 & 0.652 & 0.060 & 1000 \\
\hline M0072 & full & exp15 & 1024bit-ECFP4 & $\mathrm{RF}$ & 0.680 & 0.223 & 0.338 & 0.611 & 0.069 & 700 \\
\hline M0073 & full & exp16 & 1024bit-ECFP4 & $\mathrm{RF}$ & 0.596 & 0.267 & 0.377 & 0.660 & 0.064 & 500 \\
\hline M0074 & full & exp17 & 1024bit-ECFP4 & $\mathrm{RF}$ & 0.601 & 0.283 & 0.386 & 0.616 & 0.015 & 500 \\
\hline M0075 & full & exp18 & 1024bit-ECFP4 & $\mathrm{RF}$ & 0.645 & 0.240 & 0.375 & 0.625 & 0.020 & 500 \\
\hline M0076 & full & exp19 & 1024bit-ECFP4 & $\mathrm{RF}$ & 0.632 & 0.252 & 0.377 & 0.656 & 0.024 & 900 \\
\hline M0077 & full & exp01 & 2048bit-ECFP4 & KNN & 0.679 & 0.256 & 0.362 & 0.590 & 0.089 & 3 distance \\
\hline M0078 & full & $\exp 02$ & 2048bit-ECFP4 & KNN & 0.650 & 0.265 & 0.380 & 0.633 & 0.017 & 3 distance \\
\hline M0079 & full & $\exp 03$ & 2048bit-ECFP4 & KNN & 0.620 & 0.277 & 0.379 & 0.619 & 0.001 & 3 distance \\
\hline M0080 & full & exp04 & 2048bit-ECFP4 & KNN & 0.558 & 0.330 & 0.427 & 0.625 & 0.067 & 3 distance \\
\hline
\end{tabular}


Supporting Information (Texts S1-S9, Figures S1-S41 and Tables S1-S12)

\begin{tabular}{|c|c|c|c|c|c|c|c|c|c|c|}
\hline M0081 & full & exp05 & 2048bit-ECFP4 & KNN & 0.648 & 0.252 & 0.380 & 0.630 & 0.018 & 3 uniform \\
\hline M0082 & full & exp06 & 2048bit-ECFP4 & KNN & 0.628 & 0.272 & 0.398 & 0.607 & 0.021 & 5 distance \\
\hline M0083 & full & exp07 & 2048bit-ECFP4 & KNN & 0.583 & 0.314 & 0.401 & 0.635 & 0.052 & 3 distance \\
\hline M0084 & full & exp08 & 2048bit-ECFP4 & KNN & 0.629 & 0.273 & 0.405 & 0.632 & 0.003 & 5 distance \\
\hline M0085 & full & exp09 & 2048bit-ECFP4 & KNN & 0.500 & 0.357 & 0.431 & 0.566 & 0.066 & 7 distance \\
\hline M0086 & full & exp10 & 2048bit-ECFP4 & KNN & 0.633 & 0.271 & 0.387 & 0.608 & 0.025 & 3 distance \\
\hline M0087 & full & exp11 & 2048bit-ECFP4 & KNN & 0.621 & 0.299 & 0.402 & 0.567 & 0.054 & 3 distance \\
\hline M0088 & full & exp12 & 2048bit-ECFP4 & KNN & 0.589 & 0.305 & 0.411 & 0.620 & 0.031 & 3 distance \\
\hline M0089 & full & exp13 & 2048bit-ECFP4 & KNN & 0.654 & 0.248 & 0.374 & 0.608 & 0.046 & 3 distance \\
\hline M0090 & full & exp14 & 2048bit-ECFP4 & KNN & 0.570 & 0.339 & 0.430 & 0.661 & 0.091 & 3 distance \\
\hline M0091 & full & exp15 & 2048bit-ECFP4 & KNN & 0.648 & 0.284 & 0.371 & 0.595 & 0.053 & 3 distance \\
\hline M0092 & full & exp16 & 2048bit-ECFP4 & KNN & 0.596 & 0.295 & 0.401 & 0.632 & 0.036 & 5 distance \\
\hline M0093 & full & exp17 & 2048bit-ECFP4 & KNN & 0.597 & 0.312 & 0.403 & 0.626 & 0.029 & 3 distance \\
\hline M0094 & full & exp18 & 2048bit-ECFP4 & KNN & 0.616 & 0.288 & 0.404 & 0.608 & 0.008 & 3 distance \\
\hline M0095 & full & exp19 & 2048bit-ECFP4 & KNN & 0.566 & 0.314 & 0.410 & 0.645 & 0.079 & 7 distance \\
\hline M0096 & full & exp01 & 2048bit-ECFP4 & SVM & 0.712 & 0.201 & 0.339 & 0.642 & 0.070 & 100.01 \\
\hline M0097 & full & exp02 & 2048bit-ECFP4 & SVM & 0.665 & 0.245 & 0.366 & 0.659 & 0.006 & 100.01 \\
\hline M0098 & full & exp03 & 2048bit-ECFP4 & SVM & 0.651 & 0.242 & 0.366 & 0.659 & 0.008 & 100.01 \\
\hline M0099 & full & $\exp 04$ & 2048bit-ECFP4 & SVM & 0.629 & 0.248 & 0.377 & 0.661 & 0.032 & 1000.01 \\
\hline M0100 & full & exp05 & 2048bit-ECFP4 & SVM & 0.673 & 0.217 & 0.356 & 0.664 & 0.009 & 100.01 \\
\hline M0101 & full & exp06 & 2048bit-ECFP4 & SVM & 0.631 & 0.265 & 0.380 & 0.646 & 0.015 & 100.01 \\
\hline M0102 & full & exp07 & 2048bit-ECFP4 & SVM & 0.579 & 0.293 & 0.402 & 0.674 & 0.095 & 100.01 \\
\hline M0103 & full & exp08 & 2048bit-ECFP4 & SVM & 0.644 & 0.244 & 0.373 & 0.686 & 0.042 & 500.01 \\
\hline M0104 & full & exp09 & 2048bit-ECFP4 & SVM & 0.601 & 0.272 & 0.394 & 0.636 & 0.035 & 50.01 \\
\hline M0105 & full & exp10 & 2048bit-ECFP4 & SVM & 0.645 & 0.249 & 0.380 & 0.640 & 0.005 & 50.01 \\
\hline M0106 & full & exp11 & 2048bit-ECFP4 & SVM & 0.670 & 0.227 & 0.361 & 0.624 & 0.046 & 50.005 \\
\hline M0107 & full & exp12 & 2048bit-ECFP4 & SVM & 0.615 & 0.263 & 0.384 & 0.649 & 0.034 & 100.01 \\
\hline M0108 & full & exp13 & 2048bit-ECFP4 & SVM & 0.705 & 0.215 & 0.361 & 0.630 & 0.075 & 100.01 \\
\hline M0109 & full & exp14 & 2048bit-ECFP4 & SVM & 0.609 & 0.279 & 0.401 & 0.686 & 0.078 & 100.01 \\
\hline M0110 & full & exp15 & 2048bit-ECFP4 & SVM & 0.705 & 0.212 & 0.338 & 0.632 & 0.073 & 100.01 \\
\hline M0111 & full & exp16 & 2048bit-ECFP4 & SVM & 0.648 & 0.236 & 0.374 & 0.641 & 0.007 & 100.01 \\
\hline M0112 & full & exp17 & 2048bit-ECFP4 & SVM & 0.594 & 0.292 & 0.401 & 0.657 & 0.063 & 100.01 \\
\hline M0113 & full & exp18 & 2048bit-ECFP4 & SVM & 0.650 & 0.239 & 0.381 & 0.626 & 0.024 & 100000.00 .01 \\
\hline M0114 & full & exp19 & 2048bit-ECFP4 & SVM & 0.646 & 0.242 & 0.368 & 0.660 & 0.014 & 100.01 \\
\hline M0115 & full & exp01 & 2048bit-ECFP4 & GBM & 0.652 & 0.245 & 0.373 & 0.610 & 0.042 & 5000.1 \\
\hline M0116 & full & $\exp 02$ & 2048bit-ECFP4 & GBM & 0.701 & 0.221 & 0.351 & 0.605 & 0.096 & 6000.1 \\
\hline M0117 & full & exp03 & 2048bit-ECFP4 & GBM & 0.611 & 0.266 & 0.377 & 0.625 & 0.014 & 3000.2 \\
\hline M0118 & full & exp04 & 2048bit-ECFP4 & GBM & 0.610 & 0.263 & 0.382 & 0.613 & 0.003 & 2000.4 \\
\hline M0119 & full & exp05 & 2048bit-ECFP4 & GBM & 0.618 & 0.252 & 0.382 & 0.631 & 0.013 & 2000.1 \\
\hline M0120 & full & exp06 & 2048bit-ECFP4 & GBM & 0.620 & 0.267 & 0.387 & 0.612 & 0.008 & 3000.1 \\
\hline M0121 & full & exp07 & 2048bit-ECFP4 & GBM & 0.600 & 0.275 & 0.389 & 0.599 & 0.001 & 3000.1 \\
\hline M0122 & full & exp08 & 2048bit-ECFP4 & GBM & 0.619 & 0.265 & 0.395 & 0.653 & 0.034 & 3000.2 \\
\hline M0123 & full & exp09 & 2048bit-ECFP4 & GBM & 0.546 & 0.307 & 0.414 & 0.579 & 0.033 & 1000.2 \\
\hline M0124 & full & exp10 & 2048bit-ECFP4 & GBM & 0.620 & 0.267 & 0.381 & 0.598 & 0.022 & 2000.2 \\
\hline M0125 & full & exp11 & 2048bit-ECFP4 & GBM & 0.635 & 0.253 & 0.379 & 0.596 & 0.039 & 2000.2 \\
\hline
\end{tabular}


Supporting Information (Texts S1-S9, Figures S1-S41 and Tables S1-S12)

\begin{tabular}{|c|c|c|c|c|c|c|c|c|c|c|}
\hline M0126 & full & exp12 & 2048bit-ECFP4 & GBM & 0.517 & 0.331 & 0.434 & 0.618 & 0.101 & 3000.2 \\
\hline M0127 & full & exp13 & 2048bit-ECFP4 & GBM & 0.698 & 0.216 & 0.361 & 0.617 & 0.081 & 2000.3 \\
\hline M0128 & full & exp14 & 2048bit-ECFP4 & GBM & 0.580 & 0.309 & 0.412 & 0.647 & 0.068 & 2000.3 \\
\hline M0129 & full & exp15 & 2048bit-ECFP4 & GBM & 0.654 & 0.246 & 0.358 & 0.602 & 0.052 & 4000.1 \\
\hline M0130 & full & exp16 & 2048bit-ECFP4 & GBM & 0.570 & 0.282 & 0.390 & 0.632 & 0.062 & 2000.1 \\
\hline M0131 & full & exp17 & 2048bit-ECFP4 & GBM & 0.618 & 0.273 & 0.387 & 0.595 & 0.023 & 6000.1 \\
\hline M0132 & full & exp18 & 2048bit-ECFP4 & GBM & 0.647 & 0.241 & 0.381 & 0.594 & 0.053 & 2000.2 \\
\hline M0133 & full & exp19 & 2048bit-ECFP4 & GBM & 0.606 & 0.269 & 0.385 & 0.622 & 0.016 & 4000.1 \\
\hline M0134 & full & exp01 & 2048bit-ECFP4 & $\mathrm{RF}$ & 0.704 & 0.206 & 0.341 & 0.622 & 0.082 & 100 \\
\hline M0135 & full & exp02 & 2048bit-ECFP4 & $\mathrm{RF}$ & 0.707 & 0.218 & 0.349 & 0.634 & 0.073 & 200 \\
\hline M0136 & full & exp03 & 2048bit-ECFP4 & $\mathrm{RF}$ & 0.655 & 0.238 & 0.363 & 0.656 & 0.001 & 900 \\
\hline M0137 & full & exp04 & 2048bit-ECFP4 & $\mathrm{RF}$ & 0.622 & 0.252 & 0.375 & 0.629 & 0.007 & 900 \\
\hline M0138 & full & exp05 & 2048bit-ECFP4 & $\mathrm{RF}$ & 0.636 & 0.242 & 0.364 & 0.665 & 0.029 & 600 \\
\hline M0139 & full & exp06 & 2048bit-ECFP4 & $\mathrm{RF}$ & 0.613 & 0.274 & 0.385 & 0.647 & 0.034 & 300 \\
\hline M0140 & full & exp07 & 2048bit-ECFP4 & $\mathrm{RF}$ & 0.581 & 0.289 & 0.395 & 0.654 & 0.073 & 700 \\
\hline M0141 & full & exp08 & 2048bit-ECFP4 & $\mathrm{RF}$ & 0.626 & 0.261 & 0.382 & 0.681 & 0.055 & 100 \\
\hline M0142 & full & exp09 & 2048bit-ECFP4 & $\mathrm{RF}$ & 0.546 & 0.307 & 0.400 & 0.625 & 0.079 & 900 \\
\hline M0143 & full & exp10 & 2048bit-ECFP4 & $\mathrm{RF}$ & 0.612 & 0.271 & 0.387 & 0.624 & 0.012 & 500 \\
\hline M0144 & full & exp11 & 2048bit-ECFP4 & $\mathrm{RF}$ & 0.655 & 0.238 & 0.359 & 0.619 & 0.036 & 400 \\
\hline M0145 & full & exp12 & 2048bit-ECFP4 & $\mathrm{RF}$ & 0.602 & 0.262 & 0.383 & 0.634 & 0.032 & 500 \\
\hline M0146 & full & exp13 & 2048bit-ECFP4 & $\mathrm{RF}$ & 0.749 & 0.191 & 0.340 & 0.617 & 0.132 & 1000 \\
\hline M0147 & full & exp14 & 2048bit-ECFP4 & $\mathrm{RF}$ & 0.577 & 0.301 & 0.406 & 0.673 & 0.096 & 600 \\
\hline M0148 & full & exp15 & 2048bit-ECFP4 & $\mathrm{RF}$ & 0.678 & 0.224 & 0.334 & 0.623 & 0.055 & 700 \\
\hline M0149 & full & exp16 & 2048bit-ECFP4 & $\mathrm{RF}$ & 0.608 & 0.258 & 0.369 & 0.672 & 0.064 & 900 \\
\hline M0150 & full & exp17 & 2048bit-ECFP4 & $\mathrm{RF}$ & 0.618 & 0.272 & 0.382 & 0.622 & 0.004 & 400 \\
\hline M0151 & full & exp18 & 2048bit-ECFP4 & $\mathrm{RF}$ & 0.637 & 0.245 & 0.378 & 0.629 & 0.008 & 600 \\
\hline M0152 & full & exp19 & 2048bit-ECFP4 & $\mathrm{RF}$ & 0.639 & 0.247 & 0.374 & 0.659 & 0.020 & 800 \\
\hline M0153 & full & exp01 & 1024bit-ECFP6 & KNN & 0.615 & 0.335 & 0.416 & 0.508 & 0.107 & 5 distance \\
\hline M0154 & full & exp02 & 1024bit-ECFP6 & KNN & 0.580 & 0.321 & 0.408 & 0.563 & 0.017 & 5 distance \\
\hline M0155 & full & exp03 & 1024bit-ECFP6 & KNN & 0.593 & 0.352 & 0.419 & 0.509 & 0.084 & 5 distance \\
\hline M0156 & full & exp04 & 1024bit-ECFP6 & KNN & 0.490 & 0.444 & 0.461 & 0.552 & 0.062 & 3 distance \\
\hline M0157 & full & exp05 & 1024bit-ECFP6 & KNN & 0.499 & 0.383 & 0.469 & 0.545 & 0.046 & 13 distance \\
\hline M0158 & full & exp06 & 1024bit-ECFP6 & KNN & 0.538 & 0.397 & 0.449 & 0.546 & 0.008 & 5 distance \\
\hline M0159 & full & exp07 & 1024bit-ECFP6 & KNN & 0.498 & 0.417 & 0.451 & 0.580 & 0.082 & 5 distance \\
\hline M0160 & full & exp08 & 1024bit-ECFP6 & KNN & 0.541 & 0.411 & 0.456 & 0.568 & 0.027 & 3 distance \\
\hline M0161 & full & exp09 & 1024bit-ECFP6 & KNN & 0.406 & 0.475 & 0.491 & 0.463 & 0.056 & 13 distance \\
\hline M0162 & full & exp10 & 1024bit-ECFP6 & KNN & 0.513 & 0.434 & 0.432 & 0.571 & 0.057 & 3 distance \\
\hline M0163 & full & exp11 & 1024bit-ECFP6 & KNN & 0.600 & 0.331 & 0.413 & 0.484 & 0.116 & 3 distance \\
\hline M0164 & full & exp12 & 1024bit-ECFP6 & KNN & 0.514 & 0.465 & 0.479 & 0.558 & 0.044 & 5 distance \\
\hline M0165 & full & exp13 & 1024bit-ECFP6 & KNN & 0.552 & 0.333 & 0.407 & 0.544 & 0.008 & 5 distance \\
\hline M0166 & full & exp14 & 1024bit-ECFP6 & KNN & 0.454 & 0.494 & 0.491 & 0.573 & 0.119 & 5 distance \\
\hline M0167 & full & exp15 & 1024bit-ECFP6 & KNN & 0.538 & 0.361 & 0.404 & 0.502 & 0.036 & 13 distance \\
\hline M0168 & full & exp16 & 1024bit-ECFP6 & KNN & 0.598 & 0.314 & 0.410 & 0.552 & 0.046 & 3 distance \\
\hline M0169 & full & exp17 & 1024bit-ECFP6 & KNN & 0.543 & 0.348 & 0.436 & 0.573 & 0.030 & 7 distance \\
\hline M0170 & full & exp18 & 1024bit-ECFP6 & KNN & 0.539 & 0.391 & 0.447 & 0.534 & 0.005 & 3 distance \\
\hline
\end{tabular}


Supporting Information (Texts S1-S9, Figures S1-S41 and Tables S1-S12)

\begin{tabular}{|c|c|c|c|c|c|c|c|c|c|c|}
\hline M0171 & full & exp19 & 1024bit-ECFP6 & KNN & 0.505 & 0.425 & 0.466 & 0.544 & 0.039 & 7 distance \\
\hline M0172 & full & exp01 & 1024bit-ECFP6 & SVM & 0.713 & 0.200 & 0.343 & 0.648 & 0.065 & 50.01 \\
\hline M0173 & full & exp02 & 1024bit-ECFP6 & SVM & 0.686 & 0.230 & 0.363 & 0.653 & 0.033 & 50.01 \\
\hline M0174 & full & exp03 & 1024bit-ECFP6 & SVM & 0.634 & 0.255 & 0.382 & 0.659 & 0.025 & 50.01 \\
\hline M0175 & full & exp04 & 1024bit-ECFP6 & SVM & 0.627 & 0.247 & 0.374 & 0.652 & 0.025 & 5000.00 .01 \\
\hline M0176 & full & exp05 & 1024bit-ECFP6 & SVM & 0.670 & 0.218 & 0.355 & 0.665 & 0.005 & 100.01 \\
\hline M0177 & full & exp06 & 1024bit-ECFP6 & SVM & 0.639 & 0.256 & 0.376 & 0.658 & 0.019 & 50.01 \\
\hline M0178 & full & exp07 & 1024bit-ECFP6 & SVM & 0.578 & 0.292 & 0.405 & 0.686 & 0.108 & 50.01 \\
\hline M0179 & full & exp08 & 1024bit-ECFP6 & SVM & 0.643 & 0.242 & 0.376 & 0.698 & 0.055 & 100.01 \\
\hline M0180 & full & exp09 & 1024bit-ECFP6 & SVM & 0.601 & 0.270 & 0.389 & 0.636 & 0.035 & 50.01 \\
\hline M0181 & full & exp10 & 1024bit-ECFP6 & SVM & 0.634 & 0.256 & 0.384 & 0.644 & 0.010 & 50.01 \\
\hline M0182 & full & $\exp 11$ & 1024bit-ECFP6 & SVM & 0.663 & 0.238 & 0.367 & 0.622 & 0.041 & 10.01 \\
\hline M0183 & full & exp12 & 1024bit-ECFP6 & SVM & 0.624 & 0.252 & 0.371 & 0.645 & 0.021 & 50.01 \\
\hline M0184 & full & exp13 & 1024bit-ECFP6 & SVM & 0.732 & 0.196 & 0.342 & 0.629 & 0.103 & 50.01 \\
\hline M0185 & full & exp14 & 1024bit-ECFP6 & SVM & 0.603 & 0.281 & 0.394 & 0.695 & 0.092 & 50.01 \\
\hline M0186 & full & exp15 & 1024bit-ECFP6 & SVM & 0.709 & 0.205 & 0.328 & 0.635 & 0.074 & 50.01 \\
\hline M0187 & full & exp16 & 1024bit-ECFP6 & SVM & 0.639 & 0.245 & 0.373 & 0.655 & 0.016 & 500.01 \\
\hline M0188 & full & exp17 & 1024bit-ECFP6 & SVM & 0.610 & 0.279 & 0.397 & 0.633 & 0.023 & 100.01 \\
\hline M0189 & full & exp18 & 1024bit-ECFP6 & SVM & 0.653 & 0.234 & 0.380 & 0.636 & 0.017 & 100.01 \\
\hline M0190 & full & exp19 & 1024bit-ECFP6 & SVM & 0.649 & 0.239 & 0.363 & 0.663 & 0.014 & 50.01 \\
\hline M0191 & full & exp01 & 1024bit-ECFP6 & GBM & 0.664 & 0.234 & 0.366 & 0.601 & 0.063 & 2000.1 \\
\hline M0192 & full & exp02 & 1024bit-ECFP6 & GBM & 0.668 & 0.244 & 0.373 & 0.588 & 0.080 & 2000.1 \\
\hline M0193 & full & exp03 & 1024bit-ECFP6 & GBM & 0.603 & 0.270 & 0.380 & 0.629 & 0.026 & 2000.2 \\
\hline M0194 & full & exp04 & 1024bit-ECFP6 & GBM & 0.606 & 0.260 & 0.380 & 0.614 & 0.008 & 2000.1 \\
\hline M0195 & full & exp05 & 1024bit-ECFP6 & GBM & 0.639 & 0.239 & 0.377 & 0.614 & 0.025 & 2000.1 \\
\hline M0196 & full & exp06 & 1024bit-ECFP6 & GBM & 0.615 & 0.271 & 0.388 & 0.619 & 0.004 & 2000.1 \\
\hline M0197 & full & exp07 & 1024bit-ECFP6 & GBM & 0.578 & 0.291 & 0.411 & 0.617 & 0.039 & 3000.1 \\
\hline M0198 & full & exp08 & 1024bit-ECFP6 & GBM & 0.579 & 0.287 & 0.405 & 0.638 & 0.059 & 2000.2 \\
\hline M0199 & full & exp09 & 1024bit-ECFP6 & GBM & 0.545 & 0.308 & 0.404 & 0.608 & 0.063 & 2000.1 \\
\hline M0200 & full & $\exp 10$ & 1024bit-ECFP6 & GBM & 0.629 & 0.263 & 0.405 & 0.579 & 0.050 & 1000.1 \\
\hline M0201 & full & exp11 & 1024bit-ECFP6 & GBM & 0.648 & 0.244 & 0.366 & 0.558 & 0.090 & 2000.1 \\
\hline M0202 & full & exp12 & 1024bit-ECFP6 & GBM & 0.534 & 0.313 & 0.415 & 0.606 & 0.072 & 3000.1 \\
\hline M0203 & full & exp13 & 1024bit-ECFP6 & GBM & 0.685 & 0.225 & 0.369 & 0.595 & 0.090 & 1000.2 \\
\hline M0204 & full & exp14 & 1024bit-ECFP6 & GBM & 0.590 & 0.289 & 0.412 & 0.608 & 0.018 & 3000.1 \\
\hline M0205 & full & exp15 & 1024bit-ECFP6 & GBM & 0.686 & 0.217 & 0.351 & 0.588 & 0.098 & 3000.1 \\
\hline M0206 & full & exp16 & 1024bit-ECFP6 & GBM & 0.563 & 0.291 & 0.404 & 0.606 & 0.043 & 1000.1 \\
\hline M0207 & full & exp17 & 1024bit-ECFP6 & GBM & 0.605 & 0.281 & 0.398 & 0.572 & 0.033 & 2000.1 \\
\hline M0208 & full & exp18 & 1024bit-ECFP6 & GBM & 0.637 & 0.243 & 0.394 & 0.580 & 0.057 & 1000.1 \\
\hline M0209 & full & exp19 & 1024bit-ECFP6 & GBM & 0.614 & 0.267 & 0.391 & 0.610 & 0.004 & 1000.1 \\
\hline M0210 & full & exp01 & 1024bit-ECFP6 & $\mathrm{RF}$ & 0.681 & 0.222 & 0.349 & 0.618 & 0.064 & 600 \\
\hline M0211 & full & exp02 & 1024bit-ECFP6 & $\mathrm{RF}$ & 0.685 & 0.235 & 0.359 & 0.606 & 0.079 & 700 \\
\hline M0212 & full & exp03 & 1024bit-ECFP6 & $\mathrm{RF}$ & 0.621 & 0.261 & 0.377 & 0.653 & 0.032 & 500 \\
\hline M0213 & full & exp04 & 1024bit-ECFP6 & $\mathrm{RF}$ & 0.601 & 0.265 & 0.382 & 0.624 & 0.023 & 200 \\
\hline M0214 & full & exp05 & 1024bit-ECFP6 & $\mathrm{RF}$ & 0.658 & 0.230 & 0.360 & 0.635 & 0.023 & 600 \\
\hline M0215 & full & $\exp 06$ & 1024bit-ECFP6 & $\mathrm{RF}$ & 0.603 & 0.281 & 0.388 & 0.641 & 0.038 & 50 \\
\hline
\end{tabular}


Supporting Information (Texts S1-S9, Figures S1-S41 and Tables S1-S12)

\begin{tabular}{|c|c|c|c|c|c|c|c|c|c|c|}
\hline M0216 & full & exp07 & 1024bit-ECFP6 & $\mathrm{RF}$ & 0.579 & 0.291 & 0.402 & 0.648 & 0.069 & 600 \\
\hline M0217 & full & exp08 & 1024bit-ECFP6 & $\mathrm{RF}$ & 0.611 & 0.269 & 0.383 & 0.663 & 0.052 & 500 \\
\hline M0218 & full & exp09 & 1024bit-ECFP6 & $\mathrm{RF}$ & 0.500 & 0.339 & 0.417 & 0.607 & 0.107 & 1000 \\
\hline M0219 & full & exp10 & 1024bit-ECFP6 & $\mathrm{RF}$ & 0.631 & 0.259 & 0.391 & 0.611 & 0.020 & 500 \\
\hline M0220 & full & exp11 & 1024bit-ECFP6 & $\mathrm{RF}$ & 0.639 & 0.248 & 0.365 & 0.593 & 0.046 & 1000 \\
\hline M0221 & full & exp12 & 1024bit-ECFP6 & $\mathrm{RF}$ & 0.581 & 0.277 & 0.397 & 0.627 & 0.046 & 500 \\
\hline M0222 & full & exp13 & 1024bit-ECFP6 & $\mathrm{RF}$ & 0.738 & 0.198 & 0.343 & 0.609 & 0.129 & 1000 \\
\hline M0223 & full & exp14 & 1024bit-ECFP6 & $\mathrm{RF}$ & 0.574 & 0.299 & 0.412 & 0.642 & 0.068 & 100 \\
\hline M0224 & full & exp15 & 1024bit-ECFP6 & $\mathrm{RF}$ & 0.679 & 0.223 & 0.347 & 0.592 & 0.087 & 700 \\
\hline M0225 & full & exp16 & 1024bit-ECFP6 & $\mathrm{RF}$ & 0.594 & 0.268 & 0.373 & 0.643 & 0.049 & 800 \\
\hline M0226 & full & exp17 & 1024bit-ECFP6 & $\mathrm{RF}$ & 0.595 & 0.289 & 0.397 & 0.597 & 0.002 & 900 \\
\hline M0227 & full & exp18 & 1024bit-ECFP6 & $\mathrm{RF}$ & 0.621 & 0.257 & 0.388 & 0.609 & 0.012 & 1000 \\
\hline M0228 & full & exp19 & 1024bit-ECFP6 & $\mathrm{RF}$ & 0.634 & 0.251 & 0.373 & 0.641 & 0.007 & 300 \\
\hline M0229 & full & exp01 & 2048bit-ECFP6 & KNN & 0.608 & 0.376 & 0.417 & 0.494 & 0.113 & 3 distance \\
\hline M0230 & full & exp02 & 2048bit-ECFP6 & KNN & 0.570 & 0.332 & 0.417 & 0.555 & 0.015 & 5 distance \\
\hline M0231 & full & exp03 & 2048bit-ECFP6 & KNN & 0.567 & 0.382 & 0.417 & 0.517 & 0.050 & 3 distance \\
\hline M0232 & full & exp04 & 2048bit-ECFP6 & KNN & 0.493 & 0.437 & 0.468 & 0.558 & 0.065 & 3 distance \\
\hline M0233 & full & exp05 & 2048bit-ECFP6 & KNN & 0.506 & 0.398 & 0.471 & 0.540 & 0.034 & 11 distance \\
\hline M0234 & full & $\exp 06$ & 2048bit-ECFP6 & KNN & 0.523 & 0.429 & 0.458 & 0.539 & 0.016 & 5 distance \\
\hline M0235 & full & exp07 & 2048bit-ECFP6 & KNN & 0.488 & 0.437 & 0.468 & 0.565 & 0.077 & 5 distance \\
\hline M0236 & full & exp08 & 2048bit-ECFP6 & KNN & 0.552 & 0.398 & 0.455 & 0.560 & 0.008 & 3 distance \\
\hline M0237 & full & exp09 & 2048bit-ECFP6 & KNN & 0.397 & 0.500 & 0.502 & 0.456 & 0.059 & 13 distance \\
\hline M0238 & full & exp10 & 2048bit-ECFP6 & KNN & 0.528 & 0.420 & 0.433 & 0.566 & 0.038 & 3 distance \\
\hline M0239 & full & $\operatorname{exp11}$ & 2048bit-ECFP6 & KNN & 0.618 & 0.348 & 0.413 & 0.492 & 0.126 & 1 uniform \\
\hline M0240 & full & exp12 & 2048bit-ECFP6 & KNN & 0.508 & 0.481 & 0.489 & 0.554 & 0.046 & 5 distance \\
\hline M0241 & full & exp13 & 2048bit-ECFP6 & KNN & 0.559 & 0.339 & 0.406 & 0.541 & 0.018 & 3 distance \\
\hline M0242 & full & exp14 & 2048bit-ECFP6 & KNN & 0.454 & 0.489 & 0.495 & 0.559 & 0.105 & 5 distance \\
\hline M0243 & full & exp15 & 2048bit-ECFP6 & KNN & 0.542 & 0.359 & 0.403 & 0.501 & 0.041 & 13 distance \\
\hline M0244 & full & exp16 & 2048bit-ECFP6 & KNN & 0.566 & 0.350 & 0.421 & 0.558 & 0.008 & 3 distance \\
\hline M0245 & full & exp17 & 2048bit-ECFP6 & KNN & 0.570 & 0.332 & 0.422 & 0.562 & 0.008 & 5 distance \\
\hline M0246 & full & exp18 & 2048bit-ECFP6 & KNN & 0.534 & 0.394 & 0.445 & 0.532 & 0.002 & 3 distance \\
\hline M0247 & full & exp19 & 2048bit-ECFP6 & KNN & 0.441 & 0.479 & 0.498 & 0.543 & 0.102 & 15 distance \\
\hline M0248 & full & exp01 & 2048bit-ECFP6 & SVM & 0.719 & 0.195 & 0.338 & 0.649 & 0.070 & 50.01 \\
\hline M0249 & full & exp02 & 2048bit-ECFP6 & SVM & 0.686 & 0.230 & 0.360 & 0.660 & 0.026 & 50.01 \\
\hline M0250 & full & $\operatorname{exp03}$ & 2048bit-ECFP6 & SVM & 0.637 & 0.253 & 0.374 & 0.667 & 0.030 & 50.01 \\
\hline M0251 & full & exp04 & 2048bit-ECFP6 & SVM & 0.631 & 0.243 & 0.371 & 0.658 & 0.027 & 50000.00 .01 \\
\hline M0252 & full & exp05 & 2048bit-ECFP6 & SVM & 0.679 & 0.212 & 0.348 & 0.675 & 0.004 & 100.01 \\
\hline M0253 & full & exp06 & 2048bit-ECFP6 & SVM & 0.642 & 0.254 & 0.376 & 0.661 & 0.019 & 100.01 \\
\hline M0254 & full & exp07 & 2048bit-ECFP6 & SVM & 0.583 & 0.288 & 0.403 & 0.690 & 0.107 & 50.01 \\
\hline M0255 & full & exp08 & 2048bit-ECFP6 & SVM & 0.652 & 0.236 & 0.370 & 0.698 & 0.046 & 100.01 \\
\hline M0256 & full & exp09 & 2048bit-ECFP6 & SVM & 0.606 & 0.267 & 0.384 & 0.641 & 0.035 & 50.005 \\
\hline M0257 & full & exp10 & 2048bit-ECFP6 & SVM & 0.646 & 0.247 & 0.378 & 0.645 & 0.001 & 50.01 \\
\hline M0258 & full & exp11 & 2048bit-ECFP6 & SVM & 0.670 & 0.234 & 0.364 & 0.630 & 0.040 & 10.01 \\
\hline M0259 & full & exp12 & 2048bit-ECFP6 & SVM & 0.621 & 0.252 & 0.375 & 0.652 & 0.031 & 50.005 \\
\hline M0260 & full & exp13 & 2048bit-ECFP6 & SVM & 0.724 & 0.201 & 0.345 & 0.638 & 0.086 & 50.01 \\
\hline
\end{tabular}


Supporting Information (Texts S1-S9, Figures S1-S41 and Tables S1-S12)

\begin{tabular}{|c|c|c|c|c|c|c|c|c|c|c|}
\hline M0261 & full & exp14 & 2048bit-ECFP6 & SVM & 0.616 & 0.271 & 0.388 & 0.696 & 0.080 & 50.01 \\
\hline M0262 & full & exp15 & 2048bit-ECFP6 & SVM & 0.709 & 0.204 & 0.327 & 0.639 & 0.070 & 50.01 \\
\hline M0263 & full & exp16 & 2048bit-ECFP6 & SVM & 0.653 & 0.232 & 0.365 & 0.658 & 0.005 & 100.01 \\
\hline M0264 & full & exp17 & 2048bit-ECFP6 & SVM & 0.614 & 0.275 & 0.393 & 0.641 & 0.027 & 50.01 \\
\hline M0265 & full & exp18 & 2048bit-ECFP6 & SVM & 0.657 & 0.233 & 0.380 & 0.645 & 0.012 & 100.01 \\
\hline M0266 & full & exp19 & 2048bit-ECFP6 & SVM & 0.657 & 0.234 & 0.359 & 0.671 & 0.014 & 50.01 \\
\hline M0267 & full & exp01 & 2048bit-ECFP6 & GBM & 0.653 & 0.247 & 0.371 & 0.595 & 0.058 & 2000.2 \\
\hline M0268 & full & $\exp 02$ & 2048bit-ECFP6 & GBM & 0.668 & 0.241 & 0.370 & 0.603 & 0.065 & 500.4 \\
\hline M0269 & full & exp03 & 2048bit-ECFP6 & GBM & 0.613 & 0.270 & 0.391 & 0.645 & 0.032 & 2000.1 \\
\hline M0270 & full & exp04 & 2048bit-ECFP6 & GBM & 0.631 & 0.243 & 0.363 & 0.618 & 0.013 & 2000.1 \\
\hline M0271 & full & exp05 & 2048bit-ECFP6 & GBM & 0.626 & 0.253 & 0.387 & 0.600 & 0.026 & 500.2 \\
\hline M0272 & full & exp06 & 2048bit-ECFP6 & GBM & 0.646 & 0.248 & 0.374 & 0.600 & 0.046 & 2000.1 \\
\hline M0273 & full & exp07 & 2048bit-ECFP6 & GBM & 0.616 & 0.266 & 0.391 & 0.607 & 0.009 & 3000.1 \\
\hline M0274 & full & exp08 & 2048bit-ECFP6 & GBM & 0.586 & 0.285 & 0.394 & 0.657 & 0.071 & 6000.1 \\
\hline M0275 & full & exp09 & 2048bit-ECFP6 & GBM & 0.587 & 0.280 & 0.393 & 0.581 & 0.006 & 2000.1 \\
\hline M0276 & full & exp10 & 2048bit-ECFP6 & GBM & 0.643 & 0.251 & 0.386 & 0.574 & 0.069 & 2000.1 \\
\hline M0277 & full & exp11 & 2048bit-ECFP6 & GBM & 0.663 & 0.233 & 0.367 & 0.587 & 0.076 & 2000.1 \\
\hline M0278 & full & exp12 & 2048bit-ECFP6 & GBM & 0.543 & 0.303 & 0.415 & 0.617 & 0.074 & 2000.1 \\
\hline M0279 & full & exp13 & 2048bit-ECFP6 & GBM & 0.710 & 0.208 & 0.350 & 0.613 & 0.097 & 3000.1 \\
\hline M0280 & full & exp14 & 2048bit-ECFP6 & GBM & 0.586 & 0.294 & 0.420 & 0.625 & 0.039 & 1000.3 \\
\hline M0281 & full & exp15 & 2048bit-ECFP6 & GBM & 0.712 & 0.199 & 0.335 & 0.592 & 0.120 & 2000.1 \\
\hline M0282 & full & exp16 & 2048bit-ECFP6 & GBM & 0.564 & 0.288 & 0.399 & 0.642 & 0.078 & 1000.1 \\
\hline M0283 & full & exp17 & 2048bit-ECFP6 & GBM & 0.593 & 0.289 & 0.401 & 0.591 & 0.002 & 3000.1 \\
\hline M0284 & full & exp18 & 2048bit-ECFP6 & GBM & 0.642 & 0.240 & 0.381 & 0.577 & 0.065 & 2000.1 \\
\hline M0285 & full & exp19 & 2048bit-ECFP6 & GBM & 0.620 & 0.260 & 0.378 & 0.634 & 0.014 & 1000.2 \\
\hline M0286 & full & exp01 & 2048bit-ECFP6 & $\mathrm{RF}$ & 0.693 & 0.215 & 0.350 & 0.627 & 0.066 & 100 \\
\hline M0287 & full & $\exp 02$ & 2048bit-ECFP6 & $\mathrm{RF}$ & 0.697 & 0.226 & 0.353 & 0.624 & 0.073 & 400 \\
\hline M0288 & full & exp03 & 2048bit-ECFP6 & $\mathrm{RF}$ & 0.639 & 0.249 & 0.368 & 0.657 & 0.018 & 1000 \\
\hline M0289 & full & exp04 & 2048bit-ECFP6 & $\mathrm{RF}$ & 0.628 & 0.247 & 0.367 & 0.617 & 0.011 & 100 \\
\hline M0290 & full & exp05 & 2048bit-ECFP6 & $\mathrm{RF}$ & 0.670 & 0.223 & 0.354 & 0.654 & 0.016 & 100 \\
\hline M0291 & full & exp06 & 2048bit-ECFP6 & $\mathrm{RF}$ & 0.606 & 0.279 & 0.382 & 0.643 & 0.037 & 1000 \\
\hline M0292 & full & exp07 & 2048bit-ECFP6 & $\mathrm{RF}$ & 0.579 & 0.291 & 0.398 & 0.651 & 0.072 & 900 \\
\hline M0293 & full & exp08 & 2048bit-ECFP6 & $\mathrm{RF}$ & 0.614 & 0.268 & 0.381 & 0.670 & 0.056 & 800 \\
\hline M0294 & full & exp09 & 2048bit-ECFP6 & $\mathrm{RF}$ & 0.543 & 0.309 & 0.402 & 0.604 & 0.061 & 1000 \\
\hline M0295 & full & $\exp 10$ & 2048bit-ECFP6 & $\mathrm{RF}$ & 0.647 & 0.248 & 0.380 & 0.614 & 0.033 & 1000 \\
\hline M0296 & full & exp11 & 2048bit-ECFP6 & $\mathrm{RF}$ & 0.661 & 0.233 & 0.359 & 0.613 & 0.048 & 900 \\
\hline M0297 & full & exp12 & 2048bit-ECFP6 & $\mathrm{RF}$ & 0.578 & 0.279 & 0.393 & 0.630 & 0.052 & 600 \\
\hline M0298 & full & exp13 & 2048bit-ECFP6 & $\mathrm{RF}$ & 0.749 & 0.192 & 0.335 & 0.605 & 0.144 & 200 \\
\hline M0299 & full & exp14 & 2048bit-ECFP6 & $\mathrm{RF}$ & 0.594 & 0.285 & 0.399 & 0.660 & 0.066 & 700 \\
\hline M0300 & full & exp15 & 2048bit-ECFP6 & $\mathrm{RF}$ & 0.699 & 0.209 & 0.335 & 0.598 & 0.101 & 700 \\
\hline M0301 & full & exp16 & 2048bit-ECFP6 & $\mathrm{RF}$ & 0.592 & 0.268 & 0.370 & 0.672 & 0.081 & 500 \\
\hline M0302 & full & exp17 & 2048bit-ECFP6 & $\mathrm{RF}$ & 0.610 & 0.279 & 0.387 & 0.607 & 0.003 & 100 \\
\hline M0303 & full & exp18 & 2048bit-ECFP6 & $\mathrm{RF}$ & 0.622 & 0.257 & 0.383 & 0.603 & 0.019 & 500 \\
\hline M0304 & full & exp19 & 2048bit-ECFP6 & $\mathrm{RF}$ & 0.645 & 0.243 & 0.365 & 0.659 & 0.014 & 900 \\
\hline M0305 & 512 & exp01 & 1024bit-ECFP4 & KNN & 0.697 & 0.216 & 0.347 & 0.624 & 0.073 & 5 distance \\
\hline
\end{tabular}


Supporting Information (Texts S1-S9, Figures S1-S41 and Tables S1-S12)

\begin{tabular}{|c|c|c|c|c|c|c|c|c|c|c|}
\hline M0306 & 512 & exp02 & 1024bit-ECFP4 & KNN & 0.678 & 0.234 & 0.366 & 0.637 & 0.041 & 5 distance \\
\hline M0307 & 512 & exp03 & 1024bit-ECFP4 & KNN & 0.655 & 0.238 & 0.369 & 0.634 & 0.021 & 5 distance \\
\hline M0308 & 512 & exp04 & 1024bit-ECFP4 & KNN & 0.594 & 0.288 & 0.407 & 0.647 & 0.053 & 3 distance \\
\hline M0309 & 512 & exp05 & 1024bit-ECFP4 & KNN & 0.638 & 0.244 & 0.369 & 0.645 & 0.007 & 5 uniform \\
\hline M0310 & 512 & exp06 & 1024bit-ECFP4 & KNN & 0.619 & 0.271 & 0.390 & 0.629 & 0.010 & 7 distance \\
\hline M0311 & 512 & exp07 & 1024bit-ECFP4 & KNN & 0.600 & 0.287 & 0.393 & 0.649 & 0.049 & 5 distance \\
\hline M0312 & 512 & exp08 & 1024bit-ECFP4 & KNN & 0.634 & 0.258 & 0.394 & 0.649 & 0.015 & 5 distance \\
\hline M0313 & 512 & exp09 & 1024bit-ECFP4 & KNN & 0.576 & 0.301 & 0.398 & 0.613 & 0.037 & 3 distance \\
\hline M0314 & 512 & exp10 & 1024bit-ECFP4 & KNN & 0.637 & 0.260 & 0.384 & 0.635 & 0.002 & 3 distance \\
\hline M0315 & 512 & exp11 & 1024bit-ECFP4 & KNN & 0.633 & 0.269 & 0.389 & 0.596 & 0.037 & 5 distance \\
\hline M0316 & 512 & exp12 & 1024bit-ECFP4 & KNN & 0.615 & 0.267 & 0.393 & 0.629 & 0.014 & 3 uniform \\
\hline M0317 & 512 & exp13 & 1024bit-ECFP4 & KNN & 0.674 & 0.234 & 0.371 & 0.620 & 0.054 & 7 distance \\
\hline M0318 & 512 & $\exp 14$ & 1024bit-ECFP4 & KNN & 0.572 & 0.317 & 0.428 & 0.656 & 0.084 & 5 distance \\
\hline M0319 & 512 & $\exp 15$ & 1024bit-ECFP4 & KNN & 0.657 & 0.264 & 0.367 & 0.621 & 0.036 & 3 distance \\
\hline M0320 & 512 & $\exp 16$ & 1024bit-ECFP4 & KNN & 0.628 & 0.254 & 0.389 & 0.651 & 0.023 & 7 distance \\
\hline M0321 & 512 & exp17 & 1024bit-ECFP4 & KNN & 0.592 & 0.295 & 0.408 & 0.637 & 0.045 & 5 distance \\
\hline M0322 & 512 & exp18 & 1024bit-ECFP4 & KNN & 0.637 & 0.262 & 0.397 & 0.633 & 0.004 & 3 distance \\
\hline M0323 & 512 & $\exp 19$ & 1024bit-ECFP4 & KNN & 0.596 & 0.276 & 0.386 & 0.656 & 0.060 & 7 distance \\
\hline M0324 & 512 & exp01 & 1024bit-ECFP4 & SVM & 0.702 & 0.208 & 0.347 & 0.639 & 0.063 & 100.01 \\
\hline M0325 & 512 & exp02 & 1024bit-ECFP4 & SVM & 0.680 & 0.236 & 0.364 & 0.656 & 0.024 & 1000.05 \\
\hline M0326 & 512 & exp03 & 1024bit-ECFP4 & SVM & 0.657 & 0.240 & 0.370 & 0.661 & 0.004 & 50000.00 .05 \\
\hline M0327 & 512 & exp04 & 1024bit-ECFP4 & SVM & 0.598 & 0.269 & 0.395 & 0.666 & 0.068 & 50.005 \\
\hline M0328 & 512 & exp05 & 1024bit-ECFP4 & SVM & 0.681 & 0.214 & 0.353 & 0.671 & 0.010 & 50.05 \\
\hline M0329 & 512 & exp06 & 1024bit-ECFP4 & SVM & 0.632 & 0.258 & 0.393 & 0.649 & 0.017 & 50.05 \\
\hline M0330 & 512 & exp07 & 1024bit-ECFP4 & SVM & 0.572 & 0.294 & 0.410 & 0.682 & 0.110 & 50.05 \\
\hline M0331 & 512 & exp08 & 1024bit-ECFP4 & SVM & 0.621 & 0.258 & 0.389 & 0.683 & 0.062 & 100.01 \\
\hline M0332 & 512 & exp09 & 1024bit-ECFP4 & SVM & 0.584 & 0.282 & 0.400 & 0.631 & 0.047 & 50.05 \\
\hline M0333 & 512 & exp10 & 1024bit-ECFP4 & SVM & 0.641 & 0.251 & 0.384 & 0.649 & 0.008 & 50.01 \\
\hline M0334 & 512 & exp11 & 1024bit-ECFP4 & SVM & 0.656 & 0.236 & 0.366 & 0.625 & 0.031 & 50.005 \\
\hline M0335 & 512 & $\exp 12$ & 1024bit-ECFP4 & SVM & 0.614 & 0.264 & 0.381 & 0.638 & 0.024 & 100.01 \\
\hline M0336 & 512 & exp13 & 1024bit-ECFP4 & SVM & 0.703 & 0.218 & 0.359 & 0.628 & 0.075 & 100.01 \\
\hline M0337 & 512 & exp14 & 1024bit-ECFP4 & SVM & 0.619 & 0.268 & 0.392 & 0.681 & 0.062 & 50.05 \\
\hline M0338 & 512 & $\exp 15$ & 1024bit-ECFP4 & SVM & 0.687 & 0.219 & 0.346 & 0.629 & 0.058 & 50.05 \\
\hline M0339 & 512 & exp16 & 1024bit-ECFP4 & SVM & 0.630 & 0.246 & 0.375 & 0.655 & 0.025 & 50.05 \\
\hline M0340 & 512 & exp17 & 1024bit-ECFP4 & SVM & 0.585 & 0.299 & 0.407 & 0.667 & 0.082 & 100.01 \\
\hline M0341 & 512 & $\exp 18$ & 1024bit-ECFP4 & SVM & 0.631 & 0.249 & 0.392 & 0.644 & 0.013 & 50.05 \\
\hline M0342 & 512 & exp19 & 1024bit-ECFP4 & SVM & 0.655 & 0.236 & 0.367 & 0.671 & 0.016 & 50.05 \\
\hline M0343 & 512 & exp01 & 1024bit-ECFP4 & GBM & 0.675 & 0.226 & 0.360 & 0.602 & 0.073 & 3000.1 \\
\hline M0344 & 512 & exp02 & 1024bit-ECFP4 & GBM & 0.670 & 0.242 & 0.373 & 0.615 & 0.055 & 3000.1 \\
\hline M0345 & 512 & exp03 & 1024bit-ECFP4 & GBM & 0.632 & 0.255 & 0.372 & 0.637 & 0.005 & 2000.1 \\
\hline M0346 & 512 & exp04 & 1024bit-ECFP4 & GBM & 0.591 & 0.275 & 0.397 & 0.635 & 0.044 & 1000.3 \\
\hline M0347 & 512 & $\exp 05$ & 1024bit-ECFP4 & GBM & 0.649 & 0.232 & 0.366 & 0.645 & 0.004 & 1000.2 \\
\hline M0348 & 512 & exp06 & 1024bit-ECFP4 & GBM & 0.588 & 0.291 & 0.401 & 0.620 & 0.032 & 1000.2 \\
\hline M0349 & 512 & exp07 & 1024bit-ECFP4 & GBM & 0.574 & 0.294 & 0.415 & 0.632 & 0.058 & 4000.1 \\
\hline M0350 & 512 & exp08 & 1024bit-ECFP4 & GBM & 0.575 & 0.291 & 0.411 & 0.673 & 0.099 & 3000.1 \\
\hline
\end{tabular}


Supporting Information (Texts S1-S9, Figures S1-S41 and Tables S1-S12)

\begin{tabular}{|c|c|c|c|c|c|c|c|c|c|c|}
\hline M0351 & 512 & exp09 & 1024bit-ECFP4 & GBM & 0.555 & 0.303 & 0.401 & 0.609 & 0.053 & 3000.1 \\
\hline M0352 & 512 & exp10 & 1024bit-ECFP4 & GBM & 0.656 & 0.242 & 0.379 & 0.612 & 0.044 & 3000.1 \\
\hline M0353 & 512 & exp11 & 1024bit-ECFP4 & GBM & 0.639 & 0.255 & 0.363 & 0.601 & 0.038 & 1000.3 \\
\hline M0354 & 512 & exp12 & 1024bit-ECFP4 & GBM & 0.550 & 0.299 & 0.411 & 0.622 & 0.072 & 1000.2 \\
\hline M0355 & 512 & exp13 & 1024bit-ECFP4 & GBM & 0.683 & 0.231 & 0.371 & 0.618 & 0.065 & 1000.1 \\
\hline M0356 & 512 & exp14 & 1024bit-ECFP4 & GBM & 0.597 & 0.286 & 0.409 & 0.640 & 0.043 & 3000.1 \\
\hline M0357 & 512 & exp15 & 1024bit-ECFP4 & GBM & 0.671 & 0.229 & 0.366 & 0.615 & 0.056 & 2000.1 \\
\hline M0358 & 512 & exp16 & 1024bit-ECFP4 & GBM & 0.563 & 0.292 & 0.398 & 0.656 & 0.094 & 2000.2 \\
\hline M0359 & 512 & exp17 & 1024bit-ECFP4 & GBM & 0.619 & 0.271 & 0.392 & 0.618 & 0.001 & 3000.1 \\
\hline M0360 & 512 & exp18 & 1024bit-ECFP4 & GBM & 0.647 & 0.240 & 0.380 & 0.612 & 0.035 & 3000.1 \\
\hline M0361 & 512 & exp19 & 1024bit-ECFP4 & GBM & 0.643 & 0.246 & 0.378 & 0.632 & 0.011 & 500.3 \\
\hline M0362 & 512 & exp01 & 1024bit-ECFP4 & $\mathrm{RF}$ & 0.693 & 0.214 & 0.344 & 0.622 & 0.071 & 700 \\
\hline M0363 & 512 & $\operatorname{exp02}$ & 1024bit-ECFP4 & $\mathrm{RF}$ & 0.698 & 0.225 & 0.355 & 0.620 & 0.078 & 400 \\
\hline M0364 & 512 & exp03 & 1024bit-ECFP4 & $\mathrm{RF}$ & 0.639 & 0.247 & 0.368 & 0.655 & 0.016 & 700 \\
\hline M0365 & 512 & exp04 & 1024bit-ECFP4 & $\mathrm{RF}$ & 0.615 & 0.257 & 0.379 & 0.639 & 0.024 & 200 \\
\hline M0366 & 512 & exp05 & 1024bit-ECFP4 & $\mathrm{RF}$ & 0.660 & 0.227 & 0.357 & 0.660 & 0.000 & 200 \\
\hline M0367 & 512 & exp06 & 1024bit-ECFP4 & $\mathrm{RF}$ & 0.608 & 0.278 & 0.394 & 0.646 & 0.038 & 700 \\
\hline M0368 & 512 & exp07 & 1024bit-ECFP4 & $\mathrm{RF}$ & 0.577 & 0.291 & 0.404 & 0.652 & 0.075 & 200 \\
\hline M0369 & 512 & exp08 & 1024bit-ECFP4 & $\mathrm{RF}$ & 0.616 & 0.267 & 0.390 & 0.671 & 0.055 & 800 \\
\hline M0370 & 512 & exp09 & 1024bit-ECFP4 & $\mathrm{RF}$ & 0.525 & 0.321 & 0.411 & 0.616 & 0.091 & 400 \\
\hline M0371 & 512 & $\exp 10$ & 1024bit-ECFP4 & $\mathrm{RF}$ & 0.625 & 0.262 & 0.387 & 0.618 & 0.007 & 500 \\
\hline M0372 & 512 & exp11 & 1024bit-ECFP4 & $\mathrm{RF}$ & 0.627 & 0.257 & 0.370 & 0.598 & 0.029 & 400 \\
\hline M0373 & 512 & exp12 & 1024bit-ECFP4 & $\mathrm{RF}$ & 0.581 & 0.278 & 0.396 & 0.636 & 0.055 & 500 \\
\hline M0374 & 512 & exp13 & 1024bit-ECFP4 & $\mathrm{RF}$ & 0.732 & 0.198 & 0.346 & 0.627 & 0.105 & 900 \\
\hline M0375 & 512 & exp14 & 1024bit-ECFP4 & $\mathrm{RF}$ & 0.587 & 0.291 & 0.405 & 0.655 & 0.068 & 300 \\
\hline M0376 & 512 & exp15 & 1024bit-ECFP4 & $\mathrm{RF}$ & 0.680 & 0.223 & 0.338 & 0.613 & 0.067 & 400 \\
\hline M0377 & 512 & exp16 & 1024bit-ECFP4 & $\mathrm{RF}$ & 0.595 & 0.267 & 0.377 & 0.663 & 0.068 & 800 \\
\hline M0378 & 512 & exp17 & 1024bit-ECFP4 & $\mathrm{RF}$ & 0.601 & 0.284 & 0.387 & 0.619 & 0.018 & 400 \\
\hline M0379 & 512 & exp18 & 1024bit-ECFP4 & $\mathrm{RF}$ & 0.644 & 0.240 & 0.375 & 0.625 & 0.019 & 1000 \\
\hline M0380 & 512 & exp19 & 1024bit-ECFP4 & $\mathrm{RF}$ & 0.631 & 0.253 & 0.378 & 0.658 & 0.027 & 900 \\
\hline M0381 & 512 & exp01 & 2048bit-ECFP4 & KNN & 0.712 & 0.208 & 0.339 & 0.626 & 0.086 & 3 distance \\
\hline M0382 & 512 & exp02 & 2048bit-ECFP4 & KNN & 0.706 & 0.219 & 0.350 & 0.651 & 0.055 & 3 distance \\
\hline M0383 & 512 & exp03 & 2048bit-ECFP4 & KNN & 0.666 & 0.227 & 0.366 & 0.653 & 0.013 & 3 distance \\
\hline M0384 & 512 & exp04 & 2048bit-ECFP4 & KNN & 0.595 & 0.287 & 0.396 & 0.669 & 0.075 & 3 distance \\
\hline M0385 & 512 & exp05 & 2048bit-ECFP4 & KNN & 0.671 & 0.225 & 0.362 & 0.649 & 0.022 & 3 distance \\
\hline M0386 & 512 & exp06 & 2048bit-ECFP4 & KNN & 0.653 & 0.246 & 0.373 & 0.649 & 0.004 & 7 distance \\
\hline M0387 & 512 & exp07 & 2048bit-ECFP4 & KNN & 0.597 & 0.284 & 0.388 & 0.660 & 0.063 & 5 distance \\
\hline M0388 & 512 & exp08 & 2048bit-ECFP4 & KNN & 0.637 & 0.253 & 0.387 & 0.683 & 0.046 & 5 distance \\
\hline M0389 & 512 & exp09 & 2048bit-ECFP4 & KNN & 0.577 & 0.290 & 0.396 & 0.623 & 0.046 & 5 distance \\
\hline M0390 & 512 & exp10 & 2048bit-ECFP4 & KNN & 0.644 & 0.253 & 0.383 & 0.630 & 0.014 & 3 distance \\
\hline M0391 & 512 & exp11 & 2048bit-ECFP4 & KNN & 0.632 & 0.273 & 0.397 & 0.623 & 0.009 & 3 distance \\
\hline M0392 & 512 & exp12 & 2048bit-ECFP4 & KNN & 0.633 & 0.250 & 0.390 & 0.634 & 0.001 & 5 distance \\
\hline M0393 & 512 & exp13 & 2048bit-ECFP4 & KNN & 0.676 & 0.238 & 0.370 & 0.631 & 0.045 & 9 distance \\
\hline M0394 & 512 & exp14 & 2048bit-ECFP4 & KNN & 0.608 & 0.290 & 0.403 & 0.684 & 0.076 & 3 distance \\
\hline M0395 & 512 & exp15 & 2048bit-ECFP4 & KNN & 0.696 & 0.228 & 0.344 & 0.640 & 0.056 & 3 distance \\
\hline
\end{tabular}


Supporting Information (Texts S1-S9, Figures S1-S41 and Tables S1-S12)

\begin{tabular}{|c|c|c|c|c|c|c|c|c|c|c|}
\hline M0396 & 512 & $\exp 16$ & 2048bit-ECFP4 & KNN & 0.629 & 0.252 & 0.382 & 0.668 & 0.039 & 7 distance \\
\hline M0397 & 512 & $\exp 17$ & 2048bit-ECFP4 & KNN & 0.603 & 0.286 & 0.398 & 0.647 & 0.044 & 5 distance \\
\hline M0398 & 512 & exp18 & 2048bit-ECFP4 & KNN & 0.634 & 0.255 & 0.393 & 0.641 & 0.007 & 5 distance \\
\hline M0399 & 512 & exp19 & 2048bit-ECFP4 & KNN & 0.612 & 0.265 & 0.389 & 0.664 & 0.052 & 5 distance \\
\hline M0400 & 512 & exp01 & 2048bit-ECFP4 & SVM & 0.722 & 0.194 & 0.339 & 0.639 & 0.083 & 50.01 \\
\hline M0401 & 512 & $\exp 02$ & 2048bit-ECFP4 & SVM & 0.681 & 0.235 & 0.362 & 0.662 & 0.019 & 50.05 \\
\hline M0402 & 512 & exp03 & 2048bit-ECFP4 & SVM & 0.666 & 0.234 & 0.365 & 0.666 & 0.000 & 50.05 \\
\hline M0403 & 512 & exp04 & 2048bit-ECFP4 & SVM & 0.627 & 0.251 & 0.383 & 0.677 & 0.050 & 500.01 \\
\hline M0404 & 512 & exp05 & 2048bit-ECFP4 & SVM & 0.695 & 0.203 & 0.346 & 0.669 & 0.026 & 50.05 \\
\hline M0405 & 512 & exp06 & 2048bit-ECFP4 & SVM & 0.650 & 0.246 & 0.380 & 0.650 & 0.000 & 50.05 \\
\hline M0406 & 512 & exp07 & 2048bit-ECFP4 & SVM & 0.565 & 0.299 & 0.410 & 0.681 & 0.116 & 50.05 \\
\hline M0407 & 512 & exp08 & 2048bit-ECFP4 & SVM & 0.648 & 0.237 & 0.368 & 0.686 & 0.038 & 100000.00 .05 \\
\hline M0408 & 512 & exp09 & 2048bit-ECFP4 & SVM & 0.600 & 0.274 & 0.392 & 0.639 & 0.039 & 50.01 \\
\hline M0409 & 512 & $\exp 10$ & 2048bit-ECFP4 & SVM & 0.637 & 0.254 & 0.386 & 0.648 & 0.011 & 50.01 \\
\hline M0410 & 512 & $\exp 11$ & 2048bit-ECFP4 & SVM & 0.682 & 0.218 & 0.352 & 0.643 & 0.039 & 50.01 \\
\hline M0411 & 512 & exp12 & 2048bit-ECFP4 & SVM & 0.602 & 0.263 & 0.396 & 0.654 & 0.052 & 50.01 \\
\hline M0412 & 512 & exp13 & 2048bit-ECFP4 & SVM & 0.700 & 0.221 & 0.367 & 0.638 & 0.062 & 100.01 \\
\hline M0413 & 512 & $\exp 14$ & 2048bit-ECFP4 & SVM & 0.621 & 0.266 & 0.385 & 0.695 & 0.074 & 50.05 \\
\hline M0414 & 512 & $\exp 15$ & 2048bit-ECFP4 & SVM & 0.696 & 0.212 & 0.335 & 0.639 & 0.057 & 50.05 \\
\hline M0415 & 512 & $\exp 16$ & 2048bit-ECFP4 & SVM & 0.635 & 0.242 & 0.368 & 0.661 & 0.026 & 100000.00 .05 \\
\hline M0416 & 512 & $\exp 17$ & 2048bit-ECFP4 & SVM & 0.600 & 0.287 & 0.398 & 0.675 & 0.076 & 100.01 \\
\hline M0417 & 512 & exp18 & 2048bit-ECFP4 & SVM & 0.629 & 0.251 & 0.390 & 0.654 & 0.025 & 50.05 \\
\hline M0418 & 512 & exp19 & 2048bit-ECFP4 & SVM & 0.668 & 0.227 & 0.359 & 0.679 & 0.011 & 50.05 \\
\hline M0419 & 512 & exp01 & 2048bit-ECFP4 & GBM & 0.678 & 0.227 & 0.360 & 0.626 & 0.052 & 2000.2 \\
\hline M0420 & 512 & $\exp 02$ & 2048bit-ECFP4 & GBM & 0.700 & 0.221 & 0.356 & 0.616 & 0.084 & 2000.3 \\
\hline M0421 & 512 & exp03 & 2048bit-ECFP4 & GBM & 0.607 & 0.268 & 0.378 & 0.636 & 0.029 & 8000.1 \\
\hline M0422 & 512 & exp04 & 2048bit-ECFP4 & GBM & 0.606 & 0.265 & 0.389 & 0.631 & 0.025 & 3000.2 \\
\hline M0423 & 512 & exp05 & 2048bit-ECFP4 & GBM & 0.619 & 0.252 & 0.382 & 0.647 & 0.028 & 500.3 \\
\hline M0424 & 512 & exp06 & 2048bit-ECFP4 & GBM & 0.623 & 0.266 & 0.388 & 0.632 & 0.009 & 1000.3 \\
\hline M0425 & 512 & exp07 & 2048bit-ECFP4 & GBM & 0.598 & 0.277 & 0.385 & 0.611 & 0.013 & 3000.1 \\
\hline M0426 & 512 & exp08 & 2048bit-ECFP4 & GBM & 0.613 & 0.265 & 0.390 & 0.670 & 0.057 & 4000.1 \\
\hline M0427 & 512 & exp09 & 2048bit-ECFP4 & GBM & 0.560 & 0.299 & 0.400 & 0.605 & 0.044 & 4000.1 \\
\hline M0428 & 512 & $\exp 10$ & 2048bit-ECFP4 & GBM & 0.610 & 0.275 & 0.383 & 0.617 & 0.007 & 4000.1 \\
\hline M0429 & 512 & exp11 & 2048bit-ECFP4 & GBM & 0.630 & 0.260 & 0.379 & 0.619 & 0.011 & 1000.4 \\
\hline M0430 & 512 & $\exp 12$ & 2048bit-ECFP4 & GBM & 0.541 & 0.307 & 0.417 & 0.633 & 0.092 & 3000.1 \\
\hline M0431 & 512 & $\exp 13$ & 2048bit-ECFP4 & GBM & 0.723 & 0.202 & 0.340 & 0.627 & 0.096 & 6000.1 \\
\hline M0432 & 512 & exp14 & 2048bit-ECFP4 & GBM & 0.570 & 0.312 & 0.417 & 0.657 & 0.087 & 1000.3 \\
\hline M0433 & 512 & $\exp 15$ & 2048bit-ECFP4 & GBM & 0.661 & 0.237 & 0.355 & 0.620 & 0.041 & 1000.2 \\
\hline M0434 & 512 & $\exp 16$ & 2048bit-ECFP4 & GBM & 0.589 & 0.274 & 0.378 & 0.655 & 0.067 & 1000.4 \\
\hline M0435 & 512 & exp17 & 2048bit-ECFP4 & GBM & 0.609 & 0.279 & 0.387 & 0.623 & 0.014 & 2000.2 \\
\hline M0436 & 512 & exp18 & 2048bit-ECFP4 & GBM & 0.639 & 0.248 & 0.384 & 0.609 & 0.030 & 2000.2 \\
\hline M0437 & 512 & $\exp 19$ & 2048bit-ECFP4 & GBM & 0.595 & 0.278 & 0.387 & 0.635 & 0.040 & 6000.1 \\
\hline M0438 & 512 & exp01 & 2048bit-ECFP4 & $\mathrm{RF}$ & 0.704 & 0.206 & 0.342 & 0.623 & 0.081 & 700 \\
\hline M0439 & 512 & $\exp 02$ & 2048bit-ECFP4 & $\mathrm{RF}$ & 0.713 & 0.214 & 0.348 & 0.638 & 0.075 & 400 \\
\hline M0440 & 512 & exp03 & 2048bit-ECFP4 & $\mathrm{RF}$ & 0.651 & 0.240 & 0.364 & 0.662 & 0.011 & 100 \\
\hline
\end{tabular}


Supporting Information (Texts S1-S9, Figures S1-S41 and Tables S1-S12)

\begin{tabular}{|c|c|c|c|c|c|c|c|c|c|c|}
\hline M0441 & 512 & exp04 & 2048bit-ECFP4 & $\mathrm{RF}$ & 0.621 & 0.253 & 0.375 & 0.637 & 0.016 & 100 \\
\hline M0442 & 512 & exp05 & 2048bit-ECFP4 & $\mathrm{RF}$ & 0.636 & 0.242 & 0.363 & 0.669 & 0.033 & 600 \\
\hline M0443 & 512 & exp06 & 2048bit-ECFP4 & $\mathrm{RF}$ & 0.614 & 0.273 & 0.386 & 0.651 & 0.037 & 300 \\
\hline M0444 & 512 & exp07 & 2048bit-ECFP4 & $\mathrm{RF}$ & 0.578 & 0.292 & 0.397 & 0.658 & 0.080 & 700 \\
\hline M0445 & 512 & exp08 & 2048bit-ECFP4 & $\mathrm{RF}$ & 0.624 & 0.262 & 0.383 & 0.684 & 0.060 & 500 \\
\hline M0446 & 512 & exp09 & 2048bit-ECFP4 & $\mathrm{RF}$ & 0.543 & 0.309 & 0.400 & 0.630 & 0.087 & 600 \\
\hline M0447 & 512 & $\exp 10$ & 2048bit-ECFP4 & $\mathrm{RF}$ & 0.612 & 0.271 & 0.387 & 0.628 & 0.016 & 500 \\
\hline M0448 & 512 & $\exp 11$ & 2048bit-ECFP4 & $\mathrm{RF}$ & 0.656 & 0.237 & 0.359 & 0.623 & 0.033 & 600 \\
\hline M0449 & 512 & exp12 & 2048bit-ECFP4 & $\mathrm{RF}$ & 0.603 & 0.261 & 0.383 & 0.637 & 0.034 & 500 \\
\hline M0450 & 512 & exp13 & 2048bit-ECFP4 & $\mathrm{RF}$ & 0.748 & 0.193 & 0.340 & 0.619 & 0.129 & 900 \\
\hline M0451 & 512 & exp14 & 2048bit-ECFP4 & $\mathrm{RF}$ & 0.576 & 0.302 & 0.406 & 0.676 & 0.100 & 600 \\
\hline M0452 & 512 & exp15 & 2048bit-ECFP4 & $\mathrm{RF}$ & 0.677 & 0.226 & 0.335 & 0.629 & 0.048 & 700 \\
\hline M0453 & 512 & $\exp 16$ & 2048bit-ECFP4 & $\mathrm{RF}$ & 0.609 & 0.258 & 0.367 & 0.675 & 0.066 & 900 \\
\hline M0454 & 512 & $\exp 17$ & 2048bit-ECFP4 & $\mathrm{RF}$ & 0.615 & 0.274 & 0.382 & 0.627 & 0.012 & 400 \\
\hline M0455 & 512 & $\exp 18$ & 2048bit-ECFP4 & $\mathrm{RF}$ & 0.637 & 0.246 & 0.377 & 0.632 & 0.005 & 1000 \\
\hline M0456 & 512 & exp19 & 2048bit-ECFP4 & $\mathrm{RF}$ & 0.636 & 0.249 & 0.375 & 0.662 & 0.026 & 800 \\
\hline M0457 & 512 & exp01 & 1024bit-ECFP6 & KNN & 0.667 & 0.252 & 0.375 & 0.600 & 0.067 & 5 distance \\
\hline M0458 & 512 & exp02 & 1024bit-ECFP6 & KNN & 0.669 & 0.240 & 0.366 & 0.610 & 0.059 & 5 distance \\
\hline M0459 & 512 & $\exp 03$ & 1024bit-ECFP6 & KNN & 0.597 & 0.304 & 0.389 & 0.595 & 0.002 & 3 distance \\
\hline M0460 & 512 & exp04 & 1024bit-ECFP6 & KNN & 0.521 & 0.363 & 0.432 & 0.618 & 0.097 & 3 distance \\
\hline M0461 & 512 & exp05 & 1024bit-ECFP6 & KNN & 0.641 & 0.253 & 0.377 & 0.609 & 0.032 & 3 distance \\
\hline M0462 & 512 & exp06 & 1024bit-ECFP6 & KNN & 0.588 & 0.300 & 0.412 & 0.591 & 0.003 & 7 distance \\
\hline M0463 & 512 & exp07 & 1024bit-ECFP6 & KNN & 0.596 & 0.301 & 0.395 & 0.651 & 0.055 & 3 distance \\
\hline M0464 & 512 & exp08 & 1024bit-ECFP6 & KNN & 0.603 & 0.297 & 0.410 & 0.614 & 0.011 & 3 distance \\
\hline M0465 & 512 & exp09 & 1024bit-ECFP6 & KNN & 0.542 & 0.330 & 0.414 & 0.525 & 0.017 & 5 distance \\
\hline M0466 & 512 & $\exp 10$ & 1024bit-ECFP6 & KNN & 0.607 & 0.296 & 0.394 & 0.632 & 0.025 & 3 distance \\
\hline M0467 & 512 & $\exp 11$ & 1024bit-ECFP6 & KNN & 0.626 & 0.297 & 0.398 & 0.568 & 0.058 & 3 distance \\
\hline M0468 & 512 & exp12 & 1024bit-ECFP6 & KNN & 0.617 & 0.274 & 0.395 & 0.631 & 0.014 & 3 distance \\
\hline M0469 & 512 & exp13 & 1024bit-ECFP6 & KNN & 0.636 & 0.257 & 0.378 & 0.593 & 0.043 & 5 distance \\
\hline M0470 & 512 & $\exp 14$ & 1024bit-ECFP6 & KNN & 0.552 & 0.345 & 0.423 & 0.636 & 0.084 & 3 distance \\
\hline M0471 & 512 & $\exp 15$ & 1024bit-ECFP6 & KNN & 0.596 & 0.340 & 0.386 & 0.590 & 0.006 & 3 distance \\
\hline M0472 & 512 & $\exp 16$ & 1024bit-ECFP6 & KNN & 0.614 & 0.280 & 0.389 & 0.638 & 0.024 & 3 distance \\
\hline M0473 & 512 & $\exp 17$ & 1024bit-ECFP6 & KNN & 0.588 & 0.301 & 0.409 & 0.621 & 0.033 & 5 distance \\
\hline M0474 & 512 & exp18 & 1024bit-ECFP6 & KNN & 0.584 & 0.321 & 0.429 & 0.627 & 0.043 & 3 distance \\
\hline M0475 & 512 & $\exp 19$ & 1024bit-ECFP6 & KNN & 0.606 & 0.281 & 0.395 & 0.632 & 0.026 & 5 distance \\
\hline M0476 & 512 & exp01 & 1024bit-ECFP6 & SVM & 0.710 & 0.202 & 0.339 & 0.649 & 0.061 & 50.01 \\
\hline M0477 & 512 & $\exp 02$ & 1024bit-ECFP6 & SVM & 0.674 & 0.241 & 0.369 & 0.653 & 0.021 & 50.01 \\
\hline M0478 & 512 & exp03 & 1024bit-ECFP6 & SVM & 0.632 & 0.256 & 0.380 & 0.666 & 0.034 & 50.01 \\
\hline M0479 & 512 & exp04 & 1024bit-ECFP6 & SVM & 0.626 & 0.248 & 0.379 & 0.660 & 0.034 & 500.01 \\
\hline M0480 & 512 & exp05 & 1024bit-ECFP6 & SVM & 0.685 & 0.209 & 0.352 & 0.660 & 0.025 & 100.01 \\
\hline M0481 & 512 & exp06 & 1024bit-ECFP6 & SVM & 0.633 & 0.261 & 0.375 & 0.660 & 0.027 & 50.01 \\
\hline M0482 & 512 & exp07 & 1024bit-ECFP6 & SVM & 0.563 & 0.303 & 0.413 & 0.692 & 0.129 & 50.01 \\
\hline M0483 & 512 & $\exp 08$ & 1024bit-ECFP6 & SVM & 0.619 & 0.259 & 0.386 & 0.703 & 0.084 & 100.01 \\
\hline M0484 & 512 & exp09 & 1024bit-ECFP6 & SVM & 0.596 & 0.275 & 0.391 & 0.644 & 0.048 & 50.01 \\
\hline M0485 & 512 & $\exp 10$ & 1024bit-ECFP6 & SVM & 0.640 & 0.252 & 0.383 & 0.636 & 0.004 & 50.01 \\
\hline
\end{tabular}


Supporting Information (Texts S1-S9, Figures S1-S41 and Tables S1-S12)

\begin{tabular}{|c|c|c|c|c|c|c|c|c|c|c|}
\hline M0486 & 512 & exp11 & 1024bit-ECFP6 & SVM & 0.660 & 0.245 & 0.371 & 0.616 & 0.044 & 1000.01 \\
\hline M0487 & 512 & exp12 & 1024bit-ECFP6 & SVM & 0.610 & 0.262 & 0.378 & 0.647 & 0.037 & 50.01 \\
\hline M0488 & 512 & exp13 & 1024bit-ECFP6 & SVM & 0.720 & 0.204 & 0.355 & 0.622 & 0.098 & 50.01 \\
\hline M0489 & 512 & exp14 & 1024bit-ECFP6 & SVM & 0.593 & 0.287 & 0.397 & 0.694 & 0.101 & 50.01 \\
\hline M0490 & 512 & exp15 & 1024bit-ECFP6 & SVM & 0.718 & 0.197 & 0.322 & 0.633 & 0.085 & 50.01 \\
\hline M0491 & 512 & exp16 & 1024bit-ECFP6 & SVM & 0.627 & 0.253 & 0.373 & 0.651 & 0.024 & 500.01 \\
\hline M0492 & 512 & exp17 & 1024bit-ECFP6 & SVM & 0.610 & 0.279 & 0.394 & 0.638 & 0.028 & 100.01 \\
\hline M0493 & 512 & exp18 & 1024bit-ECFP6 & SVM & 0.652 & 0.236 & 0.378 & 0.638 & 0.014 & 100.01 \\
\hline M0494 & 512 & exp19 & 1024bit-ECFP6 & SVM & 0.646 & 0.241 & 0.362 & 0.655 & 0.009 & 50.01 \\
\hline M0495 & 512 & exp01 & 1024bit-ECFP6 & GBM & 0.681 & 0.224 & 0.352 & 0.622 & 0.059 & 3000.1 \\
\hline M0496 & 512 & exp02 & 1024bit-ECFP6 & GBM & 0.672 & 0.240 & 0.369 & 0.594 & 0.078 & 2000.1 \\
\hline M0497 & 512 & $\exp 03$ & 1024bit-ECFP6 & GBM & 0.617 & 0.263 & 0.381 & 0.649 & 0.032 & 3000.1 \\
\hline M0498 & 512 & exp04 & 1024bit-ECFP6 & GBM & 0.595 & 0.269 & 0.384 & 0.629 & 0.034 & 1000.3 \\
\hline M0499 & 512 & exp05 & 1024bit-ECFP6 & GBM & 0.654 & 0.230 & 0.368 & 0.612 & 0.042 & 1000.2 \\
\hline M0500 & 512 & exp06 & 1024bit-ECFP6 & GBM & 0.624 & 0.265 & 0.385 & 0.630 & 0.006 & 2000.1 \\
\hline M0501 & 512 & exp07 & 1024bit-ECFP6 & GBM & 0.581 & 0.289 & 0.411 & 0.635 & 0.054 & 2000.1 \\
\hline M0502 & 512 & exp08 & 1024bit-ECFP6 & GBM & 0.588 & 0.284 & 0.398 & 0.675 & 0.087 & 3000.2 \\
\hline M0503 & 512 & exp09 & 1024bit-ECFP6 & GBM & 0.550 & 0.305 & 0.398 & 0.617 & 0.067 & 2000.1 \\
\hline M0504 & 512 & exp10 & 1024bit-ECFP6 & GBM & 0.633 & 0.257 & 0.398 & 0.585 & 0.048 & 2000.1 \\
\hline M0505 & 512 & exp11 & 1024bit-ECFP6 & GBM & 0.658 & 0.237 & 0.357 & 0.577 & 0.081 & 2000.1 \\
\hline M0506 & 512 & exp12 & 1024bit-ECFP6 & GBM & 0.540 & 0.311 & 0.413 & 0.628 & 0.088 & 3000.1 \\
\hline M0507 & 512 & exp13 & 1024bit-ECFP6 & GBM & 0.695 & 0.216 & 0.363 & 0.602 & 0.093 & 500.3 \\
\hline M0508 & 512 & exp14 & 1024bit-ECFP6 & GBM & 0.591 & 0.290 & 0.402 & 0.623 & 0.032 & 2000.2 \\
\hline M0509 & 512 & $\operatorname{exp15}$ & 1024bit-ECFP6 & GBM & 0.692 & 0.213 & 0.356 & 0.599 & 0.093 & 2000.1 \\
\hline M0510 & 512 & exp16 & 1024bit-ECFP6 & GBM & 0.589 & 0.271 & 0.382 & 0.626 & 0.037 & 1000.2 \\
\hline M0511 & 512 & exp17 & 1024bit-ECFP6 & GBM & 0.611 & 0.276 & 0.398 & 0.582 & 0.029 & 2000.1 \\
\hline M0512 & 512 & exp18 & 1024bit-ECFP6 & GBM & 0.628 & 0.252 & 0.390 & 0.584 & 0.044 & 1000.2 \\
\hline M0513 & 512 & exp19 & 1024bit-ECFP6 & GBM & 0.616 & 0.262 & 0.380 & 0.630 & 0.014 & 1000.3 \\
\hline M0514 & 512 & exp01 & 1024bit-ECFP6 & $\mathrm{RF}$ & 0.683 & 0.221 & 0.347 & 0.620 & 0.063 & 700 \\
\hline M0515 & 512 & exp02 & 1024bit-ECFP6 & $\mathrm{RF}$ & 0.684 & 0.237 & 0.359 & 0.609 & 0.076 & 700 \\
\hline M0516 & 512 & exp03 & 1024bit-ECFP6 & $\mathrm{RF}$ & 0.624 & 0.259 & 0.374 & 0.658 & 0.034 & 800 \\
\hline M0517 & 512 & exp04 & 1024bit-ECFP6 & $\mathrm{RF}$ & 0.601 & 0.265 & 0.381 & 0.627 & 0.026 & 200 \\
\hline M0518 & 512 & exp05 & 1024bit-ECFP6 & $\mathrm{RF}$ & 0.660 & 0.229 & 0.358 & 0.639 & 0.021 & 600 \\
\hline M0519 & 512 & exp06 & 1024bit-ECFP6 & $\mathrm{RF}$ & 0.603 & 0.280 & 0.388 & 0.643 & 0.040 & 300 \\
\hline M0520 & 512 & exp07 & 1024bit-ECFP6 & $\mathrm{RF}$ & 0.573 & 0.296 & 0.402 & 0.653 & 0.080 & 100 \\
\hline M0521 & 512 & $\exp 08$ & 1024bit-ECFP6 & $\mathrm{RF}$ & 0.609 & 0.271 & 0.384 & 0.668 & 0.059 & 500 \\
\hline M0522 & 512 & exp09 & 1024bit-ECFP6 & $\mathrm{RF}$ & 0.499 & 0.340 & 0.417 & 0.613 & 0.114 & 1000 \\
\hline M0523 & 512 & exp10 & 1024bit-ECFP6 & $\mathrm{RF}$ & 0.629 & 0.260 & 0.392 & 0.617 & 0.012 & 500 \\
\hline M0524 & 512 & exp11 & 1024bit-ECFP6 & $\mathrm{RF}$ & 0.641 & 0.247 & 0.364 & 0.599 & 0.042 & 1000 \\
\hline M0525 & 512 & exp12 & 1024bit-ECFP6 & $\mathrm{RF}$ & 0.586 & 0.273 & 0.394 & 0.632 & 0.046 & 200 \\
\hline M0526 & 512 & exp13 & 1024bit-ECFP6 & $\mathrm{RF}$ & 0.737 & 0.199 & 0.344 & 0.614 & 0.123 & 400 \\
\hline M0527 & 512 & exp14 & 1024bit-ECFP6 & $\mathrm{RF}$ & 0.577 & 0.297 & 0.412 & 0.647 & 0.070 & 100 \\
\hline M0528 & 512 & exp15 & 1024bit-ECFP6 & $\mathrm{RF}$ & 0.680 & 0.222 & 0.347 & 0.600 & 0.080 & 700 \\
\hline M0529 & 512 & exp16 & 1024bit-ECFP6 & $\mathrm{RF}$ & 0.584 & 0.274 & 0.377 & 0.649 & 0.065 & 100 \\
\hline M0530 & 512 & exp17 & 1024bit-ECFP6 & $\mathrm{RF}$ & 0.591 & 0.292 & 0.396 & 0.602 & 0.011 & 300 \\
\hline
\end{tabular}


Supporting Information (Texts S1-S9, Figures S1-S41 and Tables S1-S12)

\begin{tabular}{|c|c|c|c|c|c|c|c|c|c|c|}
\hline M0531 & 512 & exp18 & 1024bit-ECFP6 & $\mathrm{RF}$ & 0.621 & 0.257 & 0.388 & 0.615 & 0.006 & 900 \\
\hline M0532 & 512 & exp19 & 1024bit-ECFP6 & $\mathrm{RF}$ & 0.634 & 0.251 & 0.374 & 0.645 & 0.011 & 600 \\
\hline M0533 & 512 & exp01 & 2048bit-ECFP6 & KNN & 0.690 & 0.219 & 0.357 & 0.619 & 0.071 & 5 distance \\
\hline M0534 & 512 & exp02 & 2048bit-ECFP6 & KNN & 0.689 & 0.225 & 0.358 & 0.639 & 0.050 & 5 distance \\
\hline M0535 & 512 & exp03 & 2048bit-ECFP6 & KNN & 0.699 & 0.204 & 0.346 & 0.641 & 0.058 & 5 distance \\
\hline M0536 & 512 & exp04 & 2048bit-ECFP6 & KNN & 0.595 & 0.274 & 0.389 & 0.655 & 0.060 & 7 distance \\
\hline M0537 & 512 & exp05 & 2048bit-ECFP6 & KNN & 0.599 & 0.266 & 0.370 & 0.636 & 0.037 & 9 distance \\
\hline M0538 & 512 & exp06 & 2048bit-ECFP6 & KNN & 0.617 & 0.273 & 0.389 & 0.644 & 0.027 & 7 distance \\
\hline M0539 & 512 & exp07 & 2048bit-ECFP6 & KNN & 0.594 & 0.285 & 0.391 & 0.667 & 0.073 & 5 distance \\
\hline M0540 & 512 & exp08 & 2048bit-ECFP6 & KNN & 0.613 & 0.274 & 0.398 & 0.649 & 0.036 & 3 distance \\
\hline M0541 & 512 & exp09 & 2048bit-ECFP6 & KNN & 0.580 & 0.294 & 0.398 & 0.589 & 0.009 & 3 distance \\
\hline M0542 & 512 & $\exp 10$ & 2048bit-ECFP6 & KNN & 0.638 & 0.254 & 0.379 & 0.637 & 0.001 & 5 distance \\
\hline M0543 & 512 & exp11 & 2048bit-ECFP6 & KNN & 0.654 & 0.249 & 0.375 & 0.613 & 0.041 & 5 distance \\
\hline M0544 & 512 & exp12 & 2048bit-ECFP6 & KNN & 0.643 & 0.243 & 0.378 & 0.636 & 0.007 & 5 distance \\
\hline M0545 & 512 & exp13 & 2048bit-ECFP6 & KNN & 0.704 & 0.215 & 0.355 & 0.621 & 0.083 & 7 distance \\
\hline M0546 & 512 & exp14 & 2048bit-ECFP6 & KNN & 0.591 & 0.300 & 0.404 & 0.673 & 0.082 & 3 distance \\
\hline M0547 & 512 & exp15 & 2048bit-ECFP6 & KNN & 0.667 & 0.254 & 0.358 & 0.635 & 0.032 & 3 distance \\
\hline M0548 & 512 & exp16 & 2048bit-ECFP6 & KNN & 0.619 & 0.253 & 0.382 & 0.660 & 0.041 & 7 distance \\
\hline M0549 & 512 & exp17 & 2048bit-ECFP6 & KNN & 0.596 & 0.294 & 0.405 & 0.640 & 0.044 & 3 distance \\
\hline M0550 & 512 & exp18 & 2048bit-ECFP6 & KNN & 0.630 & 0.258 & 0.391 & 0.641 & 0.011 & 5 distance \\
\hline M0551 & 512 & exp19 & 2048bit-ECFP6 & KNN & 0.572 & 0.293 & 0.400 & 0.668 & 0.096 & 9 distance \\
\hline M0552 & 512 & exp01 & 2048bit-ECFP6 & SVM & 0.728 & 0.189 & 0.332 & 0.652 & 0.076 & 50.01 \\
\hline M0553 & 512 & exp02 & 2048bit-ECFP6 & SVM & 0.678 & 0.238 & 0.366 & 0.656 & 0.022 & 50.05 \\
\hline M0554 & 512 & exp03 & 2048bit-ECFP6 & SVM & 0.596 & 0.279 & 0.386 & 0.671 & 0.075 & 50.01 \\
\hline M0555 & 512 & exp04 & 2048bit-ECFP6 & SVM & 0.657 & 0.226 & 0.365 & 0.659 & 0.002 & 50.01 \\
\hline M0556 & 512 & exp05 & 2048bit-ECFP6 & SVM & 0.670 & 0.220 & 0.354 & 0.671 & 0.001 & 50.01 \\
\hline M0557 & 512 & exp06 & 2048bit-ECFP6 & SVM & 0.642 & 0.256 & 0.382 & 0.668 & 0.026 & 100000.00 .01 \\
\hline M0558 & 512 & exp07 & 2048bit-ECFP6 & SVM & 0.553 & 0.311 & 0.422 & 0.686 & 0.133 & 50.01 \\
\hline M0559 & 512 & exp08 & 2048bit-ECFP6 & SVM & 0.623 & 0.256 & 0.389 & 0.702 & 0.079 & 100.01 \\
\hline M0560 & 512 & exp09 & 2048bit-ECFP6 & SVM & 0.612 & 0.264 & 0.382 & 0.646 & 0.034 & 50.01 \\
\hline M0561 & 512 & exp10 & 2048bit-ECFP6 & SVM & 0.643 & 0.252 & 0.388 & 0.634 & 0.009 & 50.05 \\
\hline M0562 & 512 & exp11 & 2048bit-ECFP6 & SVM & 0.679 & 0.223 & 0.353 & 0.637 & 0.042 & 50.01 \\
\hline M0563 & 512 & exp12 & 2048bit-ECFP6 & SVM & 0.606 & 0.261 & 0.383 & 0.652 & 0.046 & 50.01 \\
\hline M0564 & 512 & exp13 & 2048bit-ECFP6 & SVM & 0.715 & 0.207 & 0.353 & 0.645 & 0.070 & 100.01 \\
\hline M0565 & 512 & exp14 & 2048bit-ECFP6 & SVM & 0.603 & 0.282 & 0.386 & 0.693 & 0.090 & 100.01 \\
\hline M0566 & 512 & exp15 & 2048bit-ECFP6 & SVM & 0.721 & 0.195 & 0.319 & 0.640 & 0.081 & 50.01 \\
\hline M0567 & 512 & exp16 & 2048bit-ECFP6 & SVM & 0.640 & 0.243 & 0.365 & 0.660 & 0.020 & 100.01 \\
\hline M0568 & 512 & exp17 & 2048bit-ECFP6 & SVM & 0.598 & 0.286 & 0.400 & 0.655 & 0.057 & 50.005 \\
\hline M0569 & 512 & exp18 & 2048bit-ECFP6 & SVM & 0.638 & 0.247 & 0.384 & 0.643 & 0.005 & 100.01 \\
\hline M0570 & 512 & exp19 & 2048bit-ECFP6 & SVM & 0.669 & 0.227 & 0.359 & 0.661 & 0.008 & 50.05 \\
\hline M0571 & 512 & exp01 & 2048bit-ECFP6 & GBM & 0.662 & 0.237 & 0.367 & 0.618 & 0.044 & 500.4 \\
\hline M0572 & 512 & exp02 & 2048bit-ECFP6 & GBM & 0.688 & 0.226 & 0.358 & 0.626 & 0.062 & 2000.2 \\
\hline M0573 & 512 & exp03 & 2048bit-ECFP6 & GBM & 0.600 & 0.277 & 0.388 & 0.659 & 0.059 & 1000.2 \\
\hline M0574 & 512 & exp04 & 2048bit-ECFP6 & GBM & 0.645 & 0.235 & 0.363 & 0.616 & 0.029 & 1000.2 \\
\hline M0575 & 512 & exp05 & 2048bit-ECFP6 & GBM & 0.641 & 0.238 & 0.376 & 0.606 & 0.035 & 3000.1 \\
\hline
\end{tabular}


Supporting Information (Texts S1-S9, Figures S1-S41 and Tables S1-S12)

\begin{tabular}{|c|c|c|c|c|c|c|c|c|c|c|}
\hline M0576 & 512 & exp06 & 2048bit-ECFP6 & GBM & 0.630 & 0.261 & 0.373 & 0.623 & 0.007 & 3000.1 \\
\hline M0577 & 512 & exp07 & 2048bit-ECFP6 & GBM & 0.592 & 0.286 & 0.404 & 0.626 & 0.034 & 2000.2 \\
\hline M0578 & 512 & exp08 & 2048bit-ECFP6 & GBM & 0.568 & 0.297 & 0.403 & 0.669 & 0.101 & 3000.2 \\
\hline M0579 & 512 & exp09 & 2048bit-ECFP6 & GBM & 0.595 & 0.275 & 0.384 & 0.607 & 0.012 & 2000.2 \\
\hline M0580 & 512 & exp10 & 2048bit-ECFP6 & GBM & 0.639 & 0.255 & 0.371 & 0.574 & 0.065 & 3000.2 \\
\hline M0581 & 512 & exp11 & 2048bit-ECFP6 & GBM & 0.672 & 0.232 & 0.362 & 0.594 & 0.078 & 4000.1 \\
\hline M0582 & 512 & exp12 & 2048bit-ECFP6 & GBM & 0.543 & 0.306 & 0.420 & 0.634 & 0.091 & 3000.1 \\
\hline M0583 & 512 & exp13 & 2048bit-ECFP6 & GBM & 0.737 & 0.192 & 0.331 & 0.622 & 0.115 & 2000.2 \\
\hline M0584 & 512 & exp14 & 2048bit-ECFP6 & GBM & 0.573 & 0.304 & 0.417 & 0.657 & 0.084 & 3000.1 \\
\hline M0585 & 512 & exp15 & 2048bit-ECFP6 & GBM & 0.694 & 0.212 & 0.345 & 0.610 & 0.084 & 3000.1 \\
\hline M0586 & 512 & exp16 & 2048bit-ECFP6 & GBM & 0.572 & 0.282 & 0.397 & 0.641 & 0.069 & 1000.1 \\
\hline M0587 & 512 & exp17 & 2048bit-ECFP6 & GBM & 0.573 & 0.304 & 0.412 & 0.602 & 0.029 & 2000.1 \\
\hline M0588 & 512 & exp18 & 2048bit-ECFP6 & GBM & 0.640 & 0.242 & 0.378 & 0.596 & 0.044 & 1000.2 \\
\hline M0589 & 512 & exp19 & 2048bit-ECFP6 & GBM & 0.627 & 0.255 & 0.369 & 0.631 & 0.004 & 1000.2 \\
\hline M0590 & 512 & exp01 & 2048bit-ECFP6 & $\mathrm{RF}$ & 0.693 & 0.215 & 0.350 & 0.634 & 0.059 & 100 \\
\hline M0591 & 512 & exp02 & 2048bit-ECFP6 & $\mathrm{RF}$ & 0.696 & 0.227 & 0.354 & 0.627 & 0.069 & 1000 \\
\hline M0592 & 512 & exp03 & 2048bit-ECFP6 & $\mathrm{RF}$ & 0.642 & 0.247 & 0.367 & 0.660 & 0.018 & 1000 \\
\hline M0593 & 512 & exp04 & 2048bit-ECFP6 & $\mathrm{RF}$ & 0.631 & 0.245 & 0.365 & 0.629 & 0.002 & 100 \\
\hline M0594 & 512 & exp05 & 2048bit-ECFP6 & $\mathrm{RF}$ & 0.677 & 0.218 & 0.350 & 0.658 & 0.019 & 600 \\
\hline M0595 & 512 & exp06 & 2048bit-ECFP6 & $\mathrm{RF}$ & 0.606 & 0.279 & 0.384 & 0.648 & 0.042 & 400 \\
\hline M0596 & 512 & exp07 & 2048bit-ECFP6 & $\mathrm{RF}$ & 0.577 & 0.292 & 0.398 & 0.660 & 0.083 & 500 \\
\hline M0597 & 512 & exp08 & 2048bit-ECFP6 & $\mathrm{RF}$ & 0.610 & 0.271 & 0.383 & 0.680 & 0.070 & 1000 \\
\hline M0598 & 512 & exp09 & 2048bit-ECFP6 & $\mathrm{RF}$ & 0.544 & 0.308 & 0.400 & 0.615 & 0.071 & 1000 \\
\hline M0599 & 512 & $\operatorname{exp10}$ & 2048bit-ECFP6 & $\mathrm{RF}$ & 0.648 & 0.247 & 0.378 & 0.623 & 0.025 & 500 \\
\hline M0600 & 512 & exp11 & 2048bit-ECFP6 & $\mathrm{RF}$ & 0.665 & 0.231 & 0.357 & 0.623 & 0.042 & 900 \\
\hline M0601 & 512 & exp12 & 2048bit-ECFP6 & $\mathrm{RF}$ & 0.578 & 0.279 & 0.397 & 0.634 & 0.056 & 200 \\
\hline M0602 & 512 & exp13 & 2048bit-ECFP6 & $\mathrm{RF}$ & 0.750 & 0.192 & 0.334 & 0.615 & 0.135 & 900 \\
\hline M0603 & 512 & exp14 & 2048bit-ECFP6 & $\mathrm{RF}$ & 0.589 & 0.289 & 0.402 & 0.665 & 0.076 & 700 \\
\hline M0604 & 512 & exp15 & 2048bit-ECFP6 & $\mathrm{RF}$ & 0.699 & 0.209 & 0.334 & 0.608 & 0.091 & 700 \\
\hline M0605 & 512 & exp16 & 2048bit-ECFP6 & $\mathrm{RF}$ & 0.589 & 0.270 & 0.372 & 0.680 & 0.091 & 500 \\
\hline M0606 & 512 & exp17 & 2048bit-ECFP6 & $\mathrm{RF}$ & 0.607 & 0.281 & 0.388 & 0.618 & 0.011 & 100 \\
\hline M0607 & 512 & exp18 & 2048bit-ECFP6 & $\mathrm{RF}$ & 0.622 & 0.256 & 0.382 & 0.609 & 0.013 & 400 \\
\hline M0608 & 512 & exp19 & 2048bit-ECFP6 & $\mathrm{RF}$ & 0.643 & 0.244 & 0.365 & 0.664 & 0.021 & 900 \\
\hline M0609 & 256 & exp01 & 1024bit-ECFP4 & KNN & 0.706 & 0.205 & 0.344 & 0.626 & 0.080 & 5 distance \\
\hline M0610 & 256 & exp02 & 1024bit-ECFP4 & KNN & 0.695 & 0.224 & 0.363 & 0.637 & 0.058 & 3 distance \\
\hline M0611 & 256 & $\exp 03$ & 1024bit-ECFP4 & KNN & 0.625 & 0.252 & 0.373 & 0.642 & 0.017 & 5 distance \\
\hline M0612 & 256 & exp04 & 1024bit-ECFP4 & KNN & 0.628 & 0.254 & 0.380 & 0.646 & 0.018 & 7 distance \\
\hline M0613 & 256 & exp05 & 1024bit-ECFP4 & KNN & 0.649 & 0.235 & 0.360 & 0.644 & 0.005 & 5 distance \\
\hline M0614 & 256 & exp06 & 1024bit-ECFP4 & KNN & 0.647 & 0.252 & 0.379 & 0.658 & 0.011 & 5 distance \\
\hline M0615 & 256 & exp07 & 1024bit-ECFP4 & KNN & 0.590 & 0.292 & 0.393 & 0.663 & 0.073 & 5 distance \\
\hline M0616 & 256 & exp08 & 1024bit-ECFP4 & KNN & 0.622 & 0.262 & 0.399 & 0.667 & 0.045 & 7 distance \\
\hline M0617 & 256 & exp09 & 1024bit-ECFP4 & KNN & 0.541 & 0.321 & 0.412 & 0.627 & 0.086 & 3 distance \\
\hline M0618 & 256 & $\exp 10$ & 1024bit-ECFP4 & KNN & 0.611 & 0.274 & 0.389 & 0.630 & 0.019 & 5 distance \\
\hline M0619 & 256 & exp11 & 1024bit-ECFP4 & KNN & 0.619 & 0.273 & 0.386 & 0.614 & 0.005 & 5 distance \\
\hline M0620 & 256 & exp12 & 1024bit-ECFP4 & KNN & 0.585 & 0.275 & 0.405 & 0.613 & 0.028 & 5 uniform \\
\hline
\end{tabular}


Supporting Information (Texts S1-S9, Figures S1-S41 and Tables S1-S12)

\begin{tabular}{|c|c|c|c|c|c|c|c|c|c|c|}
\hline M0621 & 256 & exp13 & 1024bit-ECFP4 & KNN & 0.716 & 0.207 & 0.346 & 0.620 & 0.096 & 3 distance \\
\hline M0622 & 256 & exp14 & 1024bit-ECFP4 & KNN & 0.606 & 0.290 & 0.404 & 0.676 & 0.070 & 3 distance \\
\hline M0623 & 256 & exp15 & 1024bit-ECFP4 & KNN & 0.669 & 0.254 & 0.355 & 0.643 & 0.026 & 3 distance \\
\hline M0624 & 256 & exp16 & 1024bit-ECFP4 & KNN & 0.628 & 0.250 & 0.376 & 0.659 & 0.031 & 5 distance \\
\hline M0625 & 256 & exp17 & 1024bit-ECFP4 & KNN & 0.573 & 0.307 & 0.411 & 0.634 & 0.061 & 7 distance \\
\hline M0626 & 256 & exp18 & 1024bit-ECFP4 & KNN & 0.636 & 0.256 & 0.395 & 0.629 & 0.007 & 5 distance \\
\hline M0627 & 256 & exp19 & 1024bit-ECFP4 & KNN & 0.597 & 0.276 & 0.400 & 0.652 & 0.055 & 9 distance \\
\hline M0628 & 256 & exp01 & 1024bit-ECFP4 & SVM & 0.721 & 0.197 & 0.338 & 0.642 & 0.079 & 50.01 \\
\hline M0629 & 256 & exp02 & 1024bit-ECFP4 & SVM & 0.685 & 0.232 & 0.359 & 0.664 & 0.021 & 50.05 \\
\hline M0630 & 256 & exp03 & 1024bit-ECFP4 & SVM & 0.657 & 0.239 & 0.371 & 0.669 & 0.012 & 100.05 \\
\hline M0631 & 256 & exp04 & 1024bit-ECFP4 & SVM & 0.623 & 0.249 & 0.378 & 0.683 & 0.060 & 100.05 \\
\hline M0632 & 256 & exp05 & 1024bit-ECFP4 & SVM & 0.693 & 0.204 & 0.343 & 0.679 & 0.014 & 50.05 \\
\hline M0633 & 256 & exp06 & 1024bit-ECFP4 & SVM & 0.657 & 0.242 & 0.373 & 0.661 & 0.004 & 100000.00 .05 \\
\hline M0634 & 256 & exp07 & 1024bit-ECFP4 & SVM & 0.559 & 0.304 & 0.411 & 0.694 & 0.134 & 50.05 \\
\hline M0635 & 256 & exp08 & 1024bit-ECFP4 & SVM & 0.636 & 0.247 & 0.375 & 0.700 & 0.064 & 50.05 \\
\hline M0636 & 256 & exp09 & 1024bit-ECFP4 & SVM & 0.573 & 0.290 & 0.402 & 0.640 & 0.067 & 50.05 \\
\hline M0637 & 256 & exp10 & 1024bit-ECFP4 & SVM & 0.641 & 0.251 & 0.383 & 0.660 & 0.019 & 50.01 \\
\hline M0638 & 256 & exp11 & 1024bit-ECFP4 & SVM & 0.693 & 0.211 & 0.353 & 0.627 & 0.066 & 50.01 \\
\hline M0639 & 256 & exp12 & 1024bit-ECFP4 & SVM & 0.642 & 0.239 & 0.374 & 0.636 & 0.006 & 50.05 \\
\hline M0640 & 256 & exp13 & 1024bit-ECFP4 & SVM & 0.678 & 0.234 & 0.371 & 0.642 & 0.036 & 100.01 \\
\hline M0641 & 256 & exp14 & 1024bit-ECFP4 & SVM & 0.619 & 0.268 & 0.390 & 0.694 & 0.075 & 50.05 \\
\hline M0642 & 256 & exp15 & 1024bit-ECFP4 & SVM & 0.698 & 0.213 & 0.332 & 0.649 & 0.048 & 50.05 \\
\hline M0643 & 256 & exp16 & 1024bit-ECFP4 & SVM & 0.641 & 0.239 & 0.367 & 0.663 & 0.022 & 50.05 \\
\hline M0644 & 256 & exp17 & 1024bit-ECFP4 & SVM & 0.587 & 0.296 & 0.400 & 0.658 & 0.071 & 100.01 \\
\hline M0645 & 256 & exp18 & 1024bit-ECFP4 & SVM & 0.640 & 0.245 & 0.383 & 0.651 & 0.011 & 50.05 \\
\hline M0646 & 256 & exp19 & 1024bit-ECFP4 & SVM & 0.647 & 0.241 & 0.373 & 0.682 & 0.035 & 50.05 \\
\hline M0647 & 256 & exp01 & 1024bit-ECFP4 & GBM & 0.640 & 0.250 & 0.369 & 0.632 & 0.008 & 1000.2 \\
\hline M0648 & 256 & exp02 & 1024bit-ECFP4 & GBM & 0.699 & 0.220 & 0.361 & 0.633 & 0.066 & 3000.1 \\
\hline M0649 & 256 & exp03 & 1024bit-ECFP4 & GBM & 0.622 & 0.259 & 0.383 & 0.651 & 0.029 & 2000.1 \\
\hline M0650 & 256 & exp04 & 1024bit-ECFP4 & GBM & 0.612 & 0.261 & 0.388 & 0.667 & 0.055 & 1000.4 \\
\hline M0651 & 256 & exp05 & 1024bit-ECFP4 & GBM & 0.654 & 0.229 & 0.368 & 0.660 & 0.006 & 1000.2 \\
\hline M0652 & 256 & exp06 & 1024bit-ECFP4 & GBM & 0.623 & 0.268 & 0.376 & 0.649 & 0.026 & 3000.1 \\
\hline M0653 & 256 & exp07 & 1024bit-ECFP4 & GBM & 0.568 & 0.302 & 0.419 & 0.645 & 0.077 & 4000.1 \\
\hline M0654 & 256 & exp08 & 1024bit-ECFP4 & GBM & 0.570 & 0.296 & 0.412 & 0.680 & 0.110 & 1000.4 \\
\hline M0655 & 256 & exp09 & 1024bit-ECFP4 & GBM & 0.540 & 0.316 & 0.409 & 0.638 & 0.098 & 3000.1 \\
\hline M0656 & 256 & $\exp 10$ & 1024bit-ECFP4 & GBM & 0.668 & 0.233 & 0.375 & 0.618 & 0.050 & 2000.1 \\
\hline M0657 & 256 & exp11 & 1024bit-ECFP4 & GBM & 0.645 & 0.246 & 0.372 & 0.612 & 0.033 & 1000.2 \\
\hline M0658 & 256 & exp12 & 1024bit-ECFP4 & GBM & 0.540 & 0.307 & 0.412 & 0.644 & 0.104 & 2000.1 \\
\hline M0659 & 256 & exp13 & 1024bit-ECFP4 & GBM & 0.702 & 0.213 & 0.355 & 0.640 & 0.062 & 2000.1 \\
\hline M0660 & 256 & exp14 & 1024bit-ECFP4 & GBM & 0.609 & 0.280 & 0.408 & 0.652 & 0.043 & 1000.3 \\
\hline M0661 & 256 & exp15 & 1024bit-ECFP4 & GBM & 0.670 & 0.231 & 0.360 & 0.638 & 0.032 & 500.4 \\
\hline M0662 & 256 & exp16 & 1024bit-ECFP4 & GBM & 0.564 & 0.293 & 0.387 & 0.664 & 0.100 & 1000.3 \\
\hline M0663 & 256 & exp17 & 1024bit-ECFP4 & GBM & 0.615 & 0.274 & 0.399 & 0.630 & 0.015 & 500.3 \\
\hline M0664 & 256 & exp18 & 1024bit-ECFP4 & GBM & 0.662 & 0.228 & 0.372 & 0.615 & 0.047 & 2000.1 \\
\hline M0665 & 256 & $\exp 19$ & 1024bit-ECFP4 & GBM & 0.637 & 0.248 & 0.381 & 0.650 & 0.013 & 1000.2 \\
\hline
\end{tabular}


Supporting Information (Texts S1-S9, Figures S1-S41 and Tables S1-S12)

\begin{tabular}{|c|c|c|c|c|c|c|c|c|c|c|}
\hline M0666 & 256 & exp01 & 1024bit-ECFP4 & $\mathrm{RF}$ & 0.689 & 0.216 & 0.344 & 0.627 & 0.062 & 500 \\
\hline M0667 & 256 & exp02 & 1024bit-ECFP4 & $\mathrm{RF}$ & 0.699 & 0.224 & 0.355 & 0.622 & 0.077 & 300 \\
\hline M0668 & 256 & exp03 & 1024bit-ECFP4 & $\mathrm{RF}$ & 0.634 & 0.249 & 0.373 & 0.659 & 0.025 & 600 \\
\hline M0669 & 256 & exp04 & 1024bit-ECFP4 & $\mathrm{RF}$ & 0.613 & 0.259 & 0.383 & 0.650 & 0.037 & 200 \\
\hline M0670 & 256 & exp05 & 1024bit-ECFP4 & $\mathrm{RF}$ & 0.659 & 0.227 & 0.354 & 0.664 & 0.005 & 400 \\
\hline M0671 & 256 & exp06 & 1024bit-ECFP4 & $\mathrm{RF}$ & 0.615 & 0.273 & 0.391 & 0.654 & 0.039 & 700 \\
\hline M0672 & 256 & exp07 & 1024bit-ECFP4 & $\mathrm{RF}$ & 0.579 & 0.290 & 0.404 & 0.662 & 0.083 & 300 \\
\hline M0673 & 256 & exp08 & 1024bit-ECFP4 & $\mathrm{RF}$ & 0.615 & 0.269 & 0.392 & 0.672 & 0.057 & 200 \\
\hline M0674 & 256 & exp09 & 1024bit-ECFP4 & $\mathrm{RF}$ & 0.518 & 0.326 & 0.414 & 0.629 & 0.111 & 1000 \\
\hline M0675 & 256 & exp10 & 1024bit-ECFP4 & $\mathrm{RF}$ & 0.622 & 0.264 & 0.389 & 0.628 & 0.006 & 500 \\
\hline M0676 & 256 & exp11 & 1024bit-ECFP4 & $\mathrm{RF}$ & 0.630 & 0.255 & 0.371 & 0.607 & 0.023 & 200 \\
\hline M0677 & 256 & exp12 & 1024bit-ECFP4 & $\mathrm{RF}$ & 0.581 & 0.278 & 0.397 & 0.635 & 0.054 & 500 \\
\hline M0678 & 256 & exp13 & 1024bit-ECFP4 & $\mathrm{RF}$ & 0.731 & 0.198 & 0.345 & 0.632 & 0.099 & 900 \\
\hline M0679 & 256 & exp14 & 1024bit-ECFP4 & $\mathrm{RF}$ & 0.596 & 0.285 & 0.401 & 0.661 & 0.065 & 1000 \\
\hline M0680 & 256 & exp15 & 1024bit-ECFP4 & $\mathrm{RF}$ & 0.666 & 0.234 & 0.344 & 0.627 & 0.039 & 100 \\
\hline M0681 & 256 & exp16 & 1024bit-ECFP4 & $\mathrm{RF}$ & 0.595 & 0.267 & 0.376 & 0.666 & 0.071 & 800 \\
\hline M0682 & 256 & exp17 & 1024bit-ECFP4 & $\mathrm{RF}$ & 0.595 & 0.288 & 0.390 & 0.621 & 0.026 & 500 \\
\hline M0683 & 256 & exp18 & 1024bit-ECFP4 & $\mathrm{RF}$ & 0.646 & 0.240 & 0.372 & 0.631 & 0.015 & 1000 \\
\hline M0684 & 256 & exp19 & 1024bit-ECFP4 & $\mathrm{RF}$ & 0.628 & 0.254 & 0.382 & 0.667 & 0.039 & 900 \\
\hline M0685 & 256 & exp01 & 2048bit-ECFP4 & KNN & 0.704 & 0.207 & 0.346 & 0.627 & 0.077 & 7 distance \\
\hline M0686 & 256 & exp02 & 2048bit-ECFP4 & KNN & 0.696 & 0.223 & 0.355 & 0.639 & 0.057 & 5 distance \\
\hline M0687 & 256 & exp03 & 2048bit-ECFP4 & KNN & 0.673 & 0.220 & 0.353 & 0.646 & 0.027 & 5 distance \\
\hline M0688 & 256 & exp04 & 2048bit-ECFP4 & KNN & 0.626 & 0.252 & 0.375 & 0.665 & 0.039 & 7 distance \\
\hline M0689 & 256 & $\exp 05$ & 2048bit-ECFP4 & KNN & 0.655 & 0.230 & 0.357 & 0.644 & 0.011 & 5 distance \\
\hline M0690 & 256 & exp06 & 2048bit-ECFP4 & KNN & 0.651 & 0.248 & 0.374 & 0.669 & 0.018 & 5 distance \\
\hline M0691 & 256 & exp07 & 2048bit-ECFP4 & KNN & 0.579 & 0.296 & 0.402 & 0.680 & 0.101 & 5 distance \\
\hline M0692 & 256 & exp08 & 2048bit-ECFP4 & KNN & 0.638 & 0.249 & 0.389 & 0.678 & 0.040 & 7 distance \\
\hline M0693 & 256 & exp09 & 2048bit-ECFP4 & KNN & 0.558 & 0.303 & 0.399 & 0.616 & 0.058 & 5 distance \\
\hline M0694 & 256 & exp10 & 2048bit-ECFP4 & KNN & 0.649 & 0.249 & 0.380 & 0.634 & 0.015 & 3 distance \\
\hline M0695 & 256 & exp11 & 2048bit-ECFP4 & KNN & 0.640 & 0.268 & 0.397 & 0.634 & 0.006 & 3 distance \\
\hline M0696 & 256 & exp12 & 2048bit-ECFP4 & KNN & 0.594 & 0.266 & 0.401 & 0.617 & 0.023 & 5 uniform \\
\hline M0697 & 256 & exp13 & 2048bit-ECFP4 & KNN & 0.730 & 0.205 & 0.340 & 0.627 & 0.103 & 5 distance \\
\hline M0698 & 256 & exp14 & 2048bit-ECFP4 & KNN & 0.612 & 0.284 & 0.400 & 0.676 & 0.064 & 3 distance \\
\hline M0699 & 256 & exp15 & 2048bit-ECFP4 & KNN & 0.704 & 0.218 & 0.336 & 0.643 & 0.061 & 3 distance \\
\hline M0700 & 256 & exp16 & 2048bit-ECFP4 & KNN & 0.633 & 0.243 & 0.377 & 0.666 & 0.033 & 5 distance \\
\hline M0701 & 256 & exp17 & 2048bit-ECFP4 & KNN & 0.554 & 0.320 & 0.407 & 0.629 & 0.075 & 7 distance \\
\hline M0702 & 256 & exp18 & 2048bit-ECFP4 & KNN & 0.629 & 0.260 & 0.396 & 0.645 & 0.016 & 5 distance \\
\hline M0703 & 256 & exp19 & 2048bit-ECFP4 & KNN & 0.644 & 0.244 & 0.375 & 0.667 & 0.023 & 5 distance \\
\hline M0704 & 256 & exp01 & 2048bit-ECFP4 & SVM & 0.714 & 0.200 & 0.350 & 0.653 & 0.061 & 50.01 \\
\hline M0705 & 256 & exp02 & 2048bit-ECFP4 & SVM & 0.683 & 0.232 & 0.360 & 0.669 & 0.014 & 50.05 \\
\hline M0706 & 256 & exp03 & 2048bit-ECFP4 & SVM & 0.654 & 0.240 & 0.369 & 0.679 & 0.025 & 100.05 \\
\hline M0707 & 256 & exp04 & 2048bit-ECFP4 & SVM & 0.595 & 0.273 & 0.396 & 0.681 & 0.086 & 100.01 \\
\hline M0708 & 256 & exp05 & 2048bit-ECFP4 & SVM & 0.690 & 0.205 & 0.349 & 0.676 & 0.014 & 50.05 \\
\hline M0709 & 256 & exp06 & 2048bit-ECFP4 & SVM & 0.666 & 0.235 & 0.370 & 0.662 & 0.004 & 100.05 \\
\hline M0710 & 256 & exp07 & 2048bit-ECFP4 & SVM & 0.550 & 0.312 & 0.423 & 0.690 & 0.140 & 50.05 \\
\hline
\end{tabular}


Supporting Information (Texts S1-S9, Figures S1-S41 and Tables S1-S12)

\begin{tabular}{|c|c|c|c|c|c|c|c|c|c|c|}
\hline M0711 & 256 & exp08 & 2048bit-ECFP4 & SVM & 0.640 & 0.243 & 0.377 & 0.700 & 0.060 & 100.05 \\
\hline M0712 & 256 & exp09 & 2048bit-ECFP4 & SVM & 0.602 & 0.270 & 0.388 & 0.637 & 0.035 & 50.05 \\
\hline M0713 & 256 & exp10 & 2048bit-ECFP4 & SVM & 0.663 & 0.236 & 0.369 & 0.653 & 0.010 & 50.05 \\
\hline M0714 & 256 & exp11 & 2048bit-ECFP4 & SVM & 0.679 & 0.220 & 0.363 & 0.642 & 0.037 & 50.01 \\
\hline M0715 & 256 & exp12 & 2048bit-ECFP4 & SVM & 0.575 & 0.282 & 0.416 & 0.651 & 0.076 & 100.01 \\
\hline M0716 & 256 & exp13 & 2048bit-ECFP4 & SVM & 0.730 & 0.196 & 0.341 & 0.632 & 0.098 & 50.05 \\
\hline M0717 & 256 & exp14 & 2048bit-ECFP4 & SVM & 0.600 & 0.282 & 0.398 & 0.697 & 0.097 & 50.05 \\
\hline M0718 & 256 & $\exp 15$ & 2048bit-ECFP4 & SVM & 0.698 & 0.213 & 0.335 & 0.652 & 0.046 & 100.05 \\
\hline M0719 & 256 & exp16 & 2048bit-ECFP4 & SVM & 0.642 & 0.238 & 0.364 & 0.666 & 0.024 & 50.05 \\
\hline M0720 & 256 & exp17 & 2048bit-ECFP4 & SVM & 0.578 & 0.302 & 0.404 & 0.663 & 0.085 & 100.01 \\
\hline M0721 & 256 & exp18 & 2048bit-ECFP4 & SVM & 0.631 & 0.252 & 0.384 & 0.653 & 0.022 & 100.05 \\
\hline M0722 & 256 & exp19 & 2048bit-ECFP4 & SVM & 0.645 & 0.242 & 0.379 & 0.675 & 0.030 & 100000.00 .05 \\
\hline M0723 & 256 & exp01 & 2048bit-ECFP4 & GBM & 0.678 & 0.227 & 0.361 & 0.613 & 0.065 & 3000.1 \\
\hline M0724 & 256 & exp02 & 2048bit-ECFP4 & GBM & 0.700 & 0.219 & 0.355 & 0.642 & 0.058 & 2000.2 \\
\hline M0725 & 256 & exp03 & 2048bit-ECFP4 & GBM & 0.572 & 0.290 & 0.390 & 0.648 & 0.076 & 2000.3 \\
\hline M0726 & 256 & exp04 & 2048bit-ECFP4 & GBM & 0.602 & 0.267 & 0.386 & 0.657 & 0.055 & 5000.1 \\
\hline M0727 & 256 & exp05 & 2048bit-ECFP4 & GBM & 0.608 & 0.259 & 0.384 & 0.649 & 0.041 & 500.4 \\
\hline M0728 & 256 & exp06 & 2048bit-ECFP4 & GBM & 0.629 & 0.261 & 0.387 & 0.649 & 0.020 & 1000.2 \\
\hline M0729 & 256 & exp07 & 2048bit-ECFP4 & GBM & 0.589 & 0.285 & 0.397 & 0.627 & 0.038 & 4000.1 \\
\hline M0730 & 256 & exp08 & 2048bit-ECFP4 & GBM & 0.599 & 0.272 & 0.400 & 0.679 & 0.080 & 3000.1 \\
\hline M0731 & 256 & exp09 & 2048bit-ECFP4 & GBM & 0.570 & 0.292 & 0.402 & 0.610 & 0.040 & 1000.2 \\
\hline M0732 & 256 & exp10 & 2048bit-ECFP4 & GBM & 0.628 & 0.261 & 0.374 & 0.628 & 0.000 & 3000.1 \\
\hline M0733 & 256 & exp11 & 2048bit-ECFP4 & GBM & 0.661 & 0.236 & 0.369 & 0.619 & 0.042 & 1000.3 \\
\hline M0734 & 256 & $\exp 12$ & 2048bit-ECFP4 & GBM & 0.549 & 0.300 & 0.416 & 0.654 & 0.105 & 1000.2 \\
\hline M0735 & 256 & $\exp 13$ & 2048bit-ECFP4 & GBM & 0.700 & 0.215 & 0.349 & 0.645 & 0.055 & 1000.3 \\
\hline M0736 & 256 & $\exp 14$ & 2048bit-ECFP4 & GBM & 0.576 & 0.312 & 0.419 & 0.675 & 0.099 & 4000.1 \\
\hline M0737 & 256 & $\exp 15$ & 2048bit-ECFP4 & GBM & 0.647 & 0.261 & 0.373 & 0.636 & 0.011 & 5000.1 \\
\hline M0738 & 256 & exp16 & 2048bit-ECFP4 & GBM & 0.599 & 0.269 & 0.380 & 0.658 & 0.059 & 2000.2 \\
\hline M0739 & 256 & exp17 & 2048bit-ECFP4 & GBM & 0.574 & 0.310 & 0.404 & 0.660 & 0.086 & 2000.3 \\
\hline M0740 & 256 & $\exp 18$ & 2048bit-ECFP4 & GBM & 0.662 & 0.229 & 0.370 & 0.615 & 0.047 & 500.4 \\
\hline M0741 & 256 & exp19 & 2048bit-ECFP4 & GBM & 0.606 & 0.271 & 0.388 & 0.661 & 0.055 & 2000.3 \\
\hline M0742 & 256 & exp01 & 2048bit-ECFP4 & $\mathrm{RF}$ & 0.706 & 0.204 & 0.338 & 0.628 & 0.078 & 300 \\
\hline M0743 & 256 & exp02 & 2048bit-ECFP4 & $\mathrm{RF}$ & 0.712 & 0.215 & 0.347 & 0.649 & 0.063 & 300 \\
\hline M0744 & 256 & exp03 & 2048bit-ECFP4 & $\mathrm{RF}$ & 0.645 & 0.243 & 0.367 & 0.670 & 0.025 & 100 \\
\hline M0745 & 256 & $\exp 04$ & 2048bit-ECFP4 & $\mathrm{RF}$ & 0.622 & 0.252 & 0.375 & 0.647 & 0.025 & 100 \\
\hline M0746 & 256 & $\exp 05$ & 2048bit-ECFP4 & $\mathrm{RF}$ & 0.640 & 0.239 & 0.360 & 0.680 & 0.040 & 500 \\
\hline M0747 & 256 & exp06 & 2048bit-ECFP4 & $\mathrm{RF}$ & 0.619 & 0.269 & 0.383 & 0.660 & 0.041 & 200 \\
\hline M0748 & 256 & exp07 & 2048bit-ECFP4 & $\mathrm{RF}$ & 0.573 & 0.296 & 0.401 & 0.666 & 0.093 & 800 \\
\hline M0749 & 256 & exp08 & 2048bit-ECFP4 & $\mathrm{RF}$ & 0.624 & 0.259 & 0.386 & 0.689 & 0.065 & 500 \\
\hline M0750 & 256 & exp09 & 2048bit-ECFP4 & $\mathrm{RF}$ & 0.539 & 0.312 & 0.402 & 0.641 & 0.102 & 900 \\
\hline M0751 & 256 & exp10 & 2048bit-ECFP4 & $\mathrm{RF}$ & 0.617 & 0.268 & 0.384 & 0.638 & 0.021 & 500 \\
\hline M0752 & 256 & $\exp 11$ & 2048bit-ECFP4 & $\mathrm{RF}$ & 0.660 & 0.234 & 0.356 & 0.632 & 0.028 & 400 \\
\hline M0753 & 256 & $\exp 12$ & 2048bit-ECFP4 & $\mathrm{RF}$ & 0.601 & 0.263 & 0.386 & 0.639 & 0.038 & 900 \\
\hline M0754 & 256 & $\exp 13$ & 2048bit-ECFP4 & $\mathrm{RF}$ & 0.751 & 0.189 & 0.336 & 0.622 & 0.129 & 1000 \\
\hline M0755 & 256 & exp14 & 2048bit-ECFP4 & $\mathrm{RF}$ & 0.568 & 0.308 & 0.409 & 0.681 & 0.114 & 600 \\
\hline
\end{tabular}


Supporting Information (Texts S1-S9, Figures S1-S41 and Tables S1-S12)

\begin{tabular}{|c|c|c|c|c|c|c|c|c|c|c|}
\hline M0756 & 256 & $\exp 15$ & 2048bit-ECFP4 & $\mathrm{RF}$ & 0.673 & 0.229 & 0.339 & 0.636 & 0.037 & 900 \\
\hline M0757 & 256 & $\exp 16$ & 2048bit-ECFP4 & $\mathrm{RF}$ & 0.607 & 0.259 & 0.369 & 0.685 & 0.078 & 900 \\
\hline M0758 & 256 & exp17 & 2048bit-ECFP4 & $\mathrm{RF}$ & 0.608 & 0.278 & 0.382 & 0.626 & 0.018 & 700 \\
\hline M0759 & 256 & exp18 & 2048bit-ECFP4 & $\mathrm{RF}$ & 0.641 & 0.243 & 0.373 & 0.640 & 0.001 & 500 \\
\hline M0760 & 256 & exp19 & 2048bit-ECFP4 & $\mathrm{RF}$ & 0.635 & 0.250 & 0.377 & 0.674 & 0.039 & 800 \\
\hline M0761 & 256 & exp01 & 1024bit-ECFP6 & KNN & 0.683 & 0.223 & 0.361 & 0.628 & 0.055 & 5 distance \\
\hline M0762 & 256 & exp02 & 1024bit-ECFP6 & KNN & 0.690 & 0.230 & 0.361 & 0.631 & 0.059 & 7 distance \\
\hline M0763 & 256 & exp03 & 1024bit-ECFP6 & KNN & 0.667 & 0.226 & 0.365 & 0.623 & 0.044 & 9 distance \\
\hline M0764 & 256 & exp04 & 1024bit-ECFP6 & KNN & 0.602 & 0.273 & 0.392 & 0.656 & 0.054 & 5 distance \\
\hline M0765 & 256 & exp05 & 1024bit-ECFP6 & KNN & 0.653 & 0.233 & 0.360 & 0.655 & 0.002 & 5 uniform \\
\hline M0766 & 256 & exp06 & 1024bit-ECFP6 & KNN & 0.640 & 0.256 & 0.386 & 0.660 & 0.020 & 5 distance \\
\hline M0767 & 256 & exp07 & 1024bit-ECFP6 & KNN & 0.567 & 0.317 & 0.404 & 0.661 & 0.094 & 3 distance \\
\hline M0768 & 256 & exp08 & 1024bit-ECFP6 & KNN & 0.584 & 0.295 & 0.405 & 0.672 & 0.088 & 3 distance \\
\hline M0769 & 256 & exp09 & 1024bit-ECFP6 & KNN & 0.535 & 0.331 & 0.417 & 0.615 & 0.080 & 3 distance \\
\hline M0770 & 256 & $\exp 10$ & 1024bit-ECFP6 & KNN & 0.623 & 0.266 & 0.396 & 0.639 & 0.016 & 7 distance \\
\hline M0771 & 256 & exp11 & 1024bit-ECFP6 & KNN & 0.643 & 0.253 & 0.378 & 0.632 & 0.011 & 5 distance \\
\hline M0772 & 256 & exp12 & 1024bit-ECFP6 & KNN & 0.629 & 0.243 & 0.377 & 0.634 & 0.005 & 7 uniform \\
\hline M0773 & 256 & exp13 & 1024bit-ECFP6 & KNN & 0.727 & 0.203 & 0.347 & 0.632 & 0.095 & 5 distance \\
\hline M0774 & 256 & exp14 & 1024bit-ECFP6 & KNN & 0.610 & 0.284 & 0.400 & 0.674 & 0.064 & 3 distance \\
\hline M0775 & 256 & $\exp 15$ & 1024bit-ECFP6 & KNN & 0.649 & 0.265 & 0.360 & 0.638 & 0.011 & 3 distance \\
\hline M0776 & 256 & $\exp 16$ & 1024bit-ECFP6 & KNN & 0.615 & 0.254 & 0.380 & 0.669 & 0.054 & 7 distance \\
\hline M0777 & 256 & exp17 & 1024bit-ECFP6 & KNN & 0.616 & 0.280 & 0.390 & 0.629 & 0.013 & 3 distance \\
\hline M0778 & 256 & exp18 & 1024bit-ECFP6 & KNN & 0.622 & 0.267 & 0.399 & 0.641 & 0.019 & 5 distance \\
\hline M0779 & 256 & exp19 & 1024bit-ECFP6 & KNN & 0.608 & 0.267 & 0.389 & 0.657 & 0.049 & 7 distance \\
\hline M0780 & 256 & exp01 & 1024bit-ECFP6 & SVM & 0.697 & 0.211 & 0.348 & 0.655 & 0.042 & 50.01 \\
\hline M0781 & 256 & exp02 & 1024bit-ECFP6 & SVM & 0.684 & 0.234 & 0.369 & 0.660 & 0.024 & 50.05 \\
\hline M0782 & 256 & exp03 & 1024bit-ECFP6 & SVM & 0.592 & 0.279 & 0.396 & 0.671 & 0.079 & 50.01 \\
\hline M0783 & 256 & exp04 & 1024bit-ECFP6 & SVM & 0.617 & 0.252 & 0.383 & 0.659 & 0.042 & 100.05 \\
\hline M0784 & 256 & exp05 & 1024bit-ECFP6 & SVM & 0.678 & 0.215 & 0.350 & 0.666 & 0.012 & 50.05 \\
\hline M0785 & 256 & exp06 & 1024bit-ECFP6 & SVM & 0.632 & 0.267 & 0.385 & 0.664 & 0.032 & 100.01 \\
\hline M0786 & 256 & exp07 & 1024bit-ECFP6 & SVM & 0.542 & 0.314 & 0.424 & 0.690 & 0.147 & 10.05 \\
\hline M0787 & 256 & exp08 & 1024bit-ECFP6 & SVM & 0.645 & 0.241 & 0.378 & 0.706 & 0.061 & 50.05 \\
\hline M0788 & 256 & exp09 & 1024bit-ECFP6 & SVM & 0.572 & 0.293 & 0.401 & 0.646 & 0.074 & 50.01 \\
\hline M0789 & 256 & exp10 & 1024bit-ECFP6 & SVM & 0.583 & 0.293 & 0.417 & 0.642 & 0.059 & 50.01 \\
\hline M0790 & 256 & exp11 & 1024bit-ECFP6 & SVM & 0.648 & 0.248 & 0.375 & 0.631 & 0.017 & 50.05 \\
\hline M0791 & 256 & $\exp 12$ & 1024bit-ECFP6 & SVM & 0.591 & 0.276 & 0.392 & 0.639 & 0.048 & 100.01 \\
\hline M0792 & 256 & $\exp 13$ & 1024bit-ECFP6 & SVM & 0.709 & 0.207 & 0.360 & 0.638 & 0.071 & 100.01 \\
\hline M0793 & 256 & exp14 & 1024bit-ECFP6 & SVM & 0.611 & 0.273 & 0.397 & 0.679 & 0.068 & 50.05 \\
\hline M0794 & 256 & $\exp 15$ & 1024bit-ECFP6 & SVM & 0.689 & 0.222 & 0.344 & 0.640 & 0.049 & 100.01 \\
\hline M0795 & 256 & exp16 & 1024bit-ECFP6 & SVM & 0.604 & 0.263 & 0.384 & 0.672 & 0.068 & 50.05 \\
\hline M0796 & 256 & exp17 & 1024bit-ECFP6 & SVM & 0.576 & 0.305 & 0.411 & 0.636 & 0.060 & 10.05 \\
\hline M0797 & 256 & $\exp 18$ & 1024bit-ECFP6 & SVM & 0.626 & 0.251 & 0.382 & 0.645 & 0.019 & 50.05 \\
\hline M0798 & 256 & $\exp 19$ & 1024bit-ECFP6 & SVM & 0.663 & 0.231 & 0.361 & 0.662 & 0.001 & 50.05 \\
\hline M0799 & 256 & exp01 & 1024bit-ECFP6 & GBM & 0.673 & 0.228 & 0.362 & 0.632 & 0.041 & 2000.1 \\
\hline M0800 & 256 & exp02 & 1024bit-ECFP6 & GBM & 0.681 & 0.234 & 0.362 & 0.615 & 0.067 & 3000.1 \\
\hline
\end{tabular}


Supporting Information (Texts S1-S9, Figures S1-S41 and Tables S1-S12)

\begin{tabular}{|c|c|c|c|c|c|c|c|c|c|c|}
\hline M0801 & 256 & exp03 & 1024bit-ECFP6 & GBM & 0.600 & 0.276 & 0.391 & 0.660 & 0.060 & 2000.1 \\
\hline M0802 & 256 & exp04 & 1024bit-ECFP6 & GBM & 0.609 & 0.261 & 0.380 & 0.644 & 0.035 & 2000.1 \\
\hline M0803 & 256 & exp05 & 1024bit-ECFP6 & GBM & 0.643 & 0.236 & 0.371 & 0.611 & 0.032 & 4000.1 \\
\hline M0804 & 256 & exp06 & 1024bit-ECFP6 & GBM & 0.612 & 0.273 & 0.399 & 0.636 & 0.024 & 500.2 \\
\hline M0805 & 256 & exp07 & 1024bit-ECFP6 & GBM & 0.577 & 0.294 & 0.412 & 0.643 & 0.066 & 3000.1 \\
\hline M0806 & 256 & exp08 & 1024bit-ECFP6 & GBM & 0.563 & 0.301 & 0.412 & 0.665 & 0.102 & 3000.1 \\
\hline M0807 & 256 & exp09 & 1024bit-ECFP6 & GBM & 0.555 & 0.306 & 0.399 & 0.629 & 0.074 & 1000.3 \\
\hline M0808 & 256 & $\exp 10$ & 1024bit-ECFP6 & GBM & 0.619 & 0.267 & 0.403 & 0.605 & 0.014 & 500.3 \\
\hline M0809 & 256 & exp11 & 1024bit-ECFP6 & GBM & 0.662 & 0.236 & 0.359 & 0.599 & 0.063 & 3000.1 \\
\hline M0810 & 256 & exp12 & 1024bit-ECFP6 & GBM & 0.554 & 0.299 & 0.409 & 0.628 & 0.074 & 2000.1 \\
\hline M0811 & 256 & exp13 & 1024bit-ECFP6 & GBM & 0.720 & 0.201 & 0.349 & 0.614 & 0.106 & 1000.2 \\
\hline M0812 & 256 & exp14 & 1024bit-ECFP6 & GBM & 0.581 & 0.297 & 0.422 & 0.633 & 0.052 & 3000.1 \\
\hline M0813 & 256 & exp15 & 1024bit-ECFP6 & GBM & 0.688 & 0.219 & 0.353 & 0.625 & 0.062 & 1000.2 \\
\hline M0814 & 256 & exp16 & 1024bit-ECFP6 & GBM & 0.592 & 0.270 & 0.386 & 0.620 & 0.028 & 2000.1 \\
\hline M0815 & 256 & exp17 & 1024bit-ECFP6 & GBM & 0.606 & 0.281 & 0.399 & 0.600 & 0.006 & 3000.1 \\
\hline M0816 & 256 & exp18 & 1024bit-ECFP6 & GBM & 0.641 & 0.243 & 0.376 & 0.591 & 0.050 & 3000.1 \\
\hline M0817 & 256 & exp19 & 1024bit-ECFP6 & GBM & 0.622 & 0.257 & 0.374 & 0.621 & 0.001 & 1000.2 \\
\hline M0818 & 256 & exp01 & 1024bit-ECFP6 & $\mathrm{RF}$ & 0.677 & 0.225 & 0.351 & 0.628 & 0.049 & 700 \\
\hline M0819 & 256 & exp02 & 1024bit-ECFP6 & $\mathrm{RF}$ & 0.683 & 0.237 & 0.364 & 0.613 & 0.070 & 400 \\
\hline M0820 & 256 & exp03 & 1024bit-ECFP6 & $\mathrm{RF}$ & 0.612 & 0.266 & 0.379 & 0.659 & 0.047 & 500 \\
\hline M0821 & 256 & exp04 & 1024bit-ECFP6 & $\mathrm{RF}$ & 0.601 & 0.265 & 0.380 & 0.625 & 0.024 & 100 \\
\hline M0822 & 256 & exp05 & 1024bit-ECFP6 & $\mathrm{RF}$ & 0.655 & 0.233 & 0.359 & 0.636 & 0.019 & 600 \\
\hline M0823 & 256 & exp06 & 1024bit-ECFP6 & $\mathrm{RF}$ & 0.603 & 0.280 & 0.393 & 0.645 & 0.042 & 300 \\
\hline M0824 & 256 & exp07 & 1024bit-ECFP6 & $\mathrm{RF}$ & 0.566 & 0.299 & 0.408 & 0.661 & 0.095 & 700 \\
\hline M0825 & 256 & exp08 & 1024bit-ECFP6 & $\mathrm{RF}$ & 0.607 & 0.273 & 0.386 & 0.677 & 0.070 & 1000 \\
\hline M0826 & 256 & exp09 & 1024bit-ECFP6 & $\mathrm{RF}$ & 0.494 & 0.344 & 0.420 & 0.625 & 0.131 & 1000 \\
\hline M0827 & 256 & $\exp 10$ & 1024bit-ECFP6 & $\mathrm{RF}$ & 0.621 & 0.266 & 0.396 & 0.619 & 0.002 & 900 \\
\hline M0828 & 256 & exp11 & 1024bit-ECFP6 & $\mathrm{RF}$ & 0.638 & 0.249 & 0.368 & 0.607 & 0.031 & 400 \\
\hline M0829 & 256 & exp12 & 1024bit-ECFP6 & $\mathrm{RF}$ & 0.583 & 0.276 & 0.396 & 0.633 & 0.050 & 200 \\
\hline M0830 & 256 & exp13 & 1024bit-ECFP6 & $\mathrm{RF}$ & 0.735 & 0.201 & 0.344 & 0.621 & 0.114 & 400 \\
\hline M0831 & 256 & exp14 & 1024bit-ECFP6 & $\mathrm{RF}$ & 0.569 & 0.302 & 0.418 & 0.647 & 0.078 & 900 \\
\hline M0832 & 256 & exp15 & 1024bit-ECFP6 & $\mathrm{RF}$ & 0.670 & 0.229 & 0.353 & 0.603 & 0.067 & 400 \\
\hline M0833 & 256 & exp16 & 1024bit-ECFP6 & $\mathrm{RF}$ & 0.590 & 0.271 & 0.380 & 0.655 & 0.065 & 400 \\
\hline M0834 & 256 & exp17 & 1024bit-ECFP6 & $\mathrm{RF}$ & 0.587 & 0.294 & 0.401 & 0.608 & 0.021 & 1000 \\
\hline M0835 & 256 & exp18 & 1024bit-ECFP6 & $\mathrm{RF}$ & 0.619 & 0.257 & 0.389 & 0.619 & 0.000 & 1000 \\
\hline M0836 & 256 & exp19 & 1024bit-ECFP6 & $\mathrm{RF}$ & 0.634 & 0.251 & 0.372 & 0.641 & 0.007 & 700 \\
\hline M0837 & 256 & exp01 & 2048bit-ECFP6 & KNN & 0.689 & 0.219 & 0.353 & 0.642 & 0.047 & 7 distance \\
\hline M0838 & 256 & exp02 & 2048bit-ECFP6 & KNN & 0.684 & 0.232 & 0.366 & 0.648 & 0.036 & 3 distance \\
\hline M0839 & 256 & exp03 & 2048bit-ECFP6 & KNN & 0.687 & 0.212 & 0.351 & 0.650 & 0.037 & 7 distance \\
\hline M0840 & 256 & exp04 & 2048bit-ECFP6 & KNN & 0.601 & 0.268 & 0.382 & 0.677 & 0.076 & 7 distance \\
\hline M0841 & 256 & exp05 & 2048bit-ECFP6 & KNN & 0.662 & 0.227 & 0.356 & 0.674 & 0.012 & 5 uniform \\
\hline M0842 & 256 & exp06 & 2048bit-ECFP6 & KNN & 0.638 & 0.256 & 0.378 & 0.680 & 0.042 & 7 distance \\
\hline M0843 & 256 & exp07 & 2048bit-ECFP6 & KNN & 0.583 & 0.297 & 0.402 & 0.660 & 0.077 & 5 distance \\
\hline M0844 & 256 & exp08 & 2048bit-ECFP6 & KNN & 0.627 & 0.260 & 0.395 & 0.661 & 0.034 & 5 distance \\
\hline M0845 & 256 & exp09 & 2048bit-ECFP6 & KNN & 0.584 & 0.281 & 0.391 & 0.618 & 0.034 & 7 distance \\
\hline
\end{tabular}


Supporting Information (Texts S1-S9, Figures S1-S41 and Tables S1-S12)

\begin{tabular}{|c|c|c|c|c|c|c|c|c|c|c|}
\hline M0846 & 256 & exp10 & 2048bit-ECFP6 & KNN & 0.623 & 0.266 & 0.387 & 0.642 & 0.019 & 5 distance \\
\hline M0847 & 256 & exp11 & 2048bit-ECFP6 & KNN & 0.645 & 0.251 & 0.373 & 0.626 & 0.019 & 5 distance \\
\hline M0848 & 256 & exp12 & 2048bit-ECFP6 & KNN & 0.617 & 0.253 & 0.386 & 0.635 & 0.018 & 9 uniform \\
\hline M0849 & 256 & exp13 & 2048bit-ECFP6 & KNN & 0.720 & 0.214 & 0.352 & 0.648 & 0.072 & 7 distance \\
\hline M0850 & 256 & exp14 & 2048bit-ECFP6 & KNN & 0.608 & 0.282 & 0.395 & 0.690 & 0.082 & 3 distance \\
\hline M0851 & 256 & exp15 & 2048bit-ECFP6 & KNN & 0.690 & 0.217 & 0.331 & 0.634 & 0.056 & 7 distance \\
\hline M0852 & 256 & exp16 & 2048bit-ECFP6 & KNN & 0.611 & 0.260 & 0.380 & 0.682 & 0.071 & 5 distance \\
\hline M0853 & 256 & exp17 & 2048bit-ECFP6 & KNN & 0.608 & 0.284 & 0.393 & 0.644 & 0.036 & 5 distance \\
\hline M0854 & 256 & exp18 & 2048bit-ECFP6 & KNN & 0.626 & 0.262 & 0.391 & 0.661 & 0.035 & 5 distance \\
\hline M0855 & 256 & exp19 & 2048bit-ECFP6 & KNN & 0.605 & 0.272 & 0.391 & 0.678 & 0.073 & 9 distance \\
\hline M0856 & 256 & exp01 & 2048bit-ECFP6 & SVM & 0.690 & 0.223 & 0.362 & 0.654 & 0.036 & 500.01 \\
\hline M0857 & 256 & $\exp 02$ & 2048bit-ECFP6 & SVM & 0.660 & 0.251 & 0.376 & 0.661 & 0.001 & 50.05 \\
\hline M0858 & 256 & exp03 & 2048bit-ECFP6 & SVM & 0.580 & 0.287 & 0.397 & 0.680 & 0.100 & 100.01 \\
\hline M0859 & 256 & exp04 & 2048bit-ECFP6 & SVM & 0.631 & 0.243 & 0.374 & 0.667 & 0.036 & 100.05 \\
\hline M0860 & 256 & exp05 & 2048bit-ECFP6 & SVM & 0.663 & 0.223 & 0.364 & 0.683 & 0.020 & 50.05 \\
\hline M0861 & 256 & exp06 & 2048bit-ECFP6 & SVM & 0.661 & 0.238 & 0.374 & 0.662 & 0.001 & 50.05 \\
\hline M0862 & 256 & exp07 & 2048bit-ECFP6 & SVM & 0.558 & 0.305 & 0.417 & 0.682 & 0.124 & 50.05 \\
\hline M0863 & 256 & exp08 & 2048bit-ECFP6 & SVM & 0.633 & 0.248 & 0.377 & 0.699 & 0.066 & 50.05 \\
\hline M0864 & 256 & exp09 & 2048bit-ECFP6 & SVM & 0.605 & 0.267 & 0.380 & 0.650 & 0.045 & 50.01 \\
\hline M0865 & 256 & exp10 & 2048bit-ECFP6 & SVM & 0.621 & 0.266 & 0.389 & 0.646 & 0.025 & 50.01 \\
\hline M0866 & 256 & exp11 & 2048bit-ECFP6 & SVM & 0.684 & 0.217 & 0.353 & 0.648 & 0.036 & 50.01 \\
\hline M0867 & 256 & exp12 & 2048bit-ECFP6 & SVM & 0.568 & 0.288 & 0.399 & 0.668 & 0.100 & 100.01 \\
\hline M0868 & 256 & exp13 & 2048bit-ECFP6 & SVM & 0.695 & 0.217 & 0.366 & 0.640 & 0.055 & 100.01 \\
\hline M0869 & 256 & exp14 & 2048bit-ECFP6 & SVM & 0.594 & 0.285 & 0.394 & 0.684 & 0.090 & 50.05 \\
\hline M0870 & 256 & exp15 & 2048bit-ECFP6 & SVM & 0.722 & 0.195 & 0.319 & 0.637 & 0.085 & 50.01 \\
\hline M0871 & 256 & exp16 & 2048bit-ECFP6 & SVM & 0.608 & 0.262 & 0.380 & 0.668 & 0.060 & 50.05 \\
\hline M0872 & 256 & exp17 & 2048bit-ECFP6 & SVM & 0.590 & 0.291 & 0.402 & 0.656 & 0.066 & 50.01 \\
\hline M0873 & 256 & exp18 & 2048bit-ECFP6 & SVM & 0.633 & 0.248 & 0.378 & 0.650 & 0.017 & 50.05 \\
\hline M0874 & 256 & exp19 & 2048bit-ECFP6 & SVM & 0.638 & 0.247 & 0.380 & 0.664 & 0.026 & 50.05 \\
\hline M0875 & 256 & exp01 & 2048bit-ECFP6 & GBM & 0.678 & 0.227 & 0.358 & 0.627 & 0.051 & 4000.1 \\
\hline M0876 & 256 & exp02 & 2048bit-ECFP6 & GBM & 0.687 & 0.229 & 0.357 & 0.623 & 0.064 & 1000.2 \\
\hline M0877 & 256 & exp03 & 2048bit-ECFP6 & GBM & 0.608 & 0.270 & 0.380 & 0.658 & 0.050 & 2000.1 \\
\hline M0878 & 256 & exp04 & 2048bit-ECFP6 & GBM & 0.651 & 0.230 & 0.362 & 0.647 & 0.004 & 3000.1 \\
\hline M0879 & 256 & exp05 & 2048bit-ECFP6 & GBM & 0.614 & 0.255 & 0.388 & 0.628 & 0.014 & 3000.1 \\
\hline M0880 & 256 & exp06 & 2048bit-ECFP6 & GBM & 0.633 & 0.260 & 0.373 & 0.649 & 0.016 & 500.4 \\
\hline M0881 & 256 & exp07 & 2048bit-ECFP6 & GBM & 0.563 & 0.306 & 0.420 & 0.646 & 0.084 & 3000.1 \\
\hline M0882 & 256 & exp08 & 2048bit-ECFP6 & GBM & 0.560 & 0.301 & 0.419 & 0.649 & 0.089 & 3000.1 \\
\hline M0883 & 256 & exp09 & 2048bit-ECFP6 & GBM & 0.583 & 0.284 & 0.397 & 0.626 & 0.043 & 2000.2 \\
\hline M0884 & 256 & exp10 & 2048bit-ECFP6 & GBM & 0.649 & 0.246 & 0.380 & 0.602 & 0.047 & 1000.2 \\
\hline M0885 & 256 & exp11 & 2048bit-ECFP6 & GBM & 0.672 & 0.235 & 0.363 & 0.610 & 0.062 & 3000.1 \\
\hline M0886 & 256 & exp12 & 2048bit-ECFP6 & GBM & 0.525 & 0.316 & 0.423 & 0.648 & 0.123 & 2000.1 \\
\hline M0887 & 256 & exp13 & 2048bit-ECFP6 & GBM & 0.716 & 0.203 & 0.348 & 0.638 & 0.078 & 1000.5 \\
\hline M0888 & 256 & exp14 & 2048bit-ECFP6 & GBM & 0.581 & 0.298 & 0.417 & 0.655 & 0.075 & 3000.1 \\
\hline M0889 & 256 & exp15 & 2048bit-ECFP6 & GBM & 0.669 & 0.232 & 0.365 & 0.628 & 0.041 & 500.4 \\
\hline M0890 & 256 & exp16 & 2048bit-ECFP6 & GBM & 0.563 & 0.288 & 0.389 & 0.663 & 0.101 & 2000.1 \\
\hline
\end{tabular}


Supporting Information (Texts S1-S9, Figures S1-S41 and Tables S1-S12)

\begin{tabular}{|c|c|c|c|c|c|c|c|c|c|c|}
\hline M0891 & 256 & $\exp 17$ & 2048bit-ECFP6 & GBM & 0.567 & 0.313 & 0.413 & 0.608 & 0.041 & 1000.5 \\
\hline M0892 & 256 & $\exp 18$ & 2048bit-ECFP6 & GBM & 0.631 & 0.253 & 0.380 & 0.616 & 0.015 & 1000.3 \\
\hline M0893 & 256 & exp19 & 2048bit-ECFP6 & GBM & 0.583 & 0.286 & 0.389 & 0.629 & 0.046 & 1000.3 \\
\hline M0894 & 256 & exp01 & 2048bit-ECFP6 & $\mathrm{RF}$ & 0.698 & 0.210 & 0.342 & 0.640 & 0.058 & 200 \\
\hline M0895 & 256 & exp02 & 2048bit-ECFP6 & $\mathrm{RF}$ & 0.693 & 0.229 & 0.352 & 0.634 & 0.059 & 300 \\
\hline M0896 & 256 & exp03 & 2048bit-ECFP6 & $\mathrm{RF}$ & 0.632 & 0.252 & 0.367 & 0.658 & 0.026 & 600 \\
\hline M0897 & 256 & exp04 & 2048bit-ECFP6 & $\mathrm{RF}$ & 0.626 & 0.247 & 0.364 & 0.636 & 0.010 & 100 \\
\hline M0898 & 256 & exp05 & 2048bit-ECFP6 & $\mathrm{RF}$ & 0.667 & 0.226 & 0.360 & 0.664 & 0.003 & 600 \\
\hline M0899 & 256 & exp06 & 2048bit-ECFP6 & $\mathrm{RF}$ & 0.598 & 0.285 & 0.389 & 0.653 & 0.055 & 500 \\
\hline M0900 & 256 & exp07 & 2048bit-ECFP6 & $\mathrm{RF}$ & 0.564 & 0.302 & 0.402 & 0.664 & 0.100 & 500 \\
\hline M0901 & 256 & exp08 & 2048bit-ECFP6 & $\mathrm{RF}$ & 0.602 & 0.277 & 0.387 & 0.686 & 0.084 & 200 \\
\hline M0902 & 256 & exp09 & 2048bit-ECFP6 & $\mathrm{RF}$ & 0.537 & 0.313 & 0.402 & 0.626 & 0.089 & 1000 \\
\hline M0903 & 256 & $\exp 10$ & 2048bit-ECFP6 & $\mathrm{RF}$ & 0.642 & 0.251 & 0.378 & 0.625 & 0.017 & 1000 \\
\hline M0904 & 256 & exp11 & 2048bit-ECFP6 & $\mathrm{RF}$ & 0.661 & 0.234 & 0.359 & 0.630 & 0.031 & 900 \\
\hline M0905 & 256 & $\exp 12$ & 2048bit-ECFP6 & $\mathrm{RF}$ & 0.575 & 0.281 & 0.395 & 0.641 & 0.066 & 500 \\
\hline M0906 & 256 & exp13 & 2048bit-ECFP6 & $\mathrm{RF}$ & 0.743 & 0.194 & 0.336 & 0.630 & 0.113 & 500 \\
\hline M0907 & 256 & exp14 & 2048bit-ECFP6 & $\mathrm{RF}$ & 0.576 & 0.297 & 0.409 & 0.667 & 0.092 & 1000 \\
\hline M0908 & 256 & exp15 & 2048bit-ECFP6 & $\mathrm{RF}$ & 0.692 & 0.213 & 0.337 & 0.610 & 0.082 & 700 \\
\hline M0909 & 256 & $\exp 16$ & 2048bit-ECFP6 & $\mathrm{RF}$ & 0.571 & 0.281 & 0.380 & 0.691 & 0.120 & 500 \\
\hline M0910 & 256 & $\exp 17$ & 2048bit-ECFP6 & $\mathrm{RF}$ & 0.596 & 0.289 & 0.390 & 0.623 & 0.027 & 100 \\
\hline M0911 & 256 & $\exp 18$ & 2048bit-ECFP6 & $\mathrm{RF}$ & 0.623 & 0.255 & 0.383 & 0.617 & 0.006 & 400 \\
\hline M0912 & 256 & exp19 & 2048bit-ECFP6 & $\mathrm{RF}$ & 0.641 & 0.246 & 0.369 & 0.665 & 0.024 & 900 \\
\hline M0913 & 128 & $\exp 01$ & 1024bit-ECFP4 & KNN & 0.648 & 0.250 & 0.372 & 0.613 & 0.035 & 7 distance \\
\hline M0914 & 128 & $\exp 02$ & 1024bit-ECFP4 & KNN & 0.664 & 0.255 & 0.379 & 0.626 & 0.038 & 3 uniform \\
\hline M0915 & 128 & $\operatorname{exp03}$ & 1024bit-ECFP4 & KNN & 0.603 & 0.266 & 0.380 & 0.631 & 0.028 & 5 distance \\
\hline M0916 & 128 & exp04 & 1024bit-ECFP4 & KNN & 0.603 & 0.272 & 0.390 & 0.632 & 0.029 & 5 distance \\
\hline M0917 & 128 & exp05 & 1024bit-ECFP4 & KNN & 0.605 & 0.266 & 0.381 & 0.647 & 0.042 & 5 uniform \\
\hline M0918 & 128 & exp06 & 1024bit-ECFP4 & KNN & 0.612 & 0.279 & 0.395 & 0.641 & 0.029 & 7 distance \\
\hline M0919 & 128 & exp07 & 1024bit-ECFP4 & KNN & 0.549 & 0.323 & 0.422 & 0.627 & 0.078 & 5 distance \\
\hline M0920 & 128 & $\exp 08$ & 1024bit-ECFP4 & KNN & 0.574 & 0.304 & 0.420 & 0.650 & 0.076 & 5 distance \\
\hline M0921 & 128 & exp09 & 1024bit-ECFP4 & KNN & 0.506 & 0.341 & 0.416 & 0.622 & 0.116 & 5 distance \\
\hline M0922 & 128 & $\exp 10$ & 1024bit-ECFP4 & KNN & 0.556 & 0.312 & 0.415 & 0.630 & 0.074 & 5 distance \\
\hline M0923 & 128 & exp11 & 1024bit-ECFP4 & KNN & 0.596 & 0.287 & 0.399 & 0.605 & 0.009 & 5 distance \\
\hline M0924 & 128 & exp12 & 1024bit-ECFP4 & KNN & 0.573 & 0.288 & 0.412 & 0.606 & 0.033 & 3 distance \\
\hline M0925 & 128 & $\exp 13$ & 1024bit-ECFP4 & KNN & 0.708 & 0.218 & 0.366 & 0.619 & 0.089 & 3 distance \\
\hline M0926 & 128 & $\exp 14$ & 1024bit-ECFP4 & KNN & 0.589 & 0.304 & 0.420 & 0.656 & 0.067 & 3 distance \\
\hline M0927 & 128 & $\exp 15$ & 1024bit-ECFP4 & KNN & 0.630 & 0.264 & 0.373 & 0.613 & 0.017 & 5 distance \\
\hline M0928 & 128 & $\exp 16$ & 1024bit-ECFP4 & KNN & 0.559 & 0.290 & 0.412 & 0.631 & 0.072 & 7 distance \\
\hline M0929 & 128 & $\exp 17$ & 1024bit-ECFP4 & KNN & 0.556 & 0.318 & 0.416 & 0.603 & 0.047 & 7 uniform \\
\hline M0930 & 128 & exp18 & 1024bit-ECFP4 & KNN & 0.616 & 0.266 & 0.404 & 0.623 & 0.007 & 5 uniform \\
\hline M0931 & 128 & exp19 & 1024bit-ECFP4 & KNN & 0.571 & 0.295 & 0.411 & 0.623 & 0.052 & 7 distance \\
\hline M0932 & 128 & exp01 & 1024bit-ECFP4 & SVM & 0.710 & 0.218 & 0.355 & 0.632 & 0.078 & 10.05 \\
\hline M0933 & 128 & $\exp 02$ & 1024bit-ECFP4 & SVM & 0.662 & 0.251 & 0.380 & 0.655 & 0.007 & 50.05 \\
\hline M0934 & 128 & $\operatorname{exp03}$ & 1024bit-ECFP4 & SVM & 0.639 & 0.250 & 0.381 & 0.657 & 0.018 & 50.1 \\
\hline M0935 & 128 & exp04 & 1024bit-ECFP4 & SVM & 0.595 & 0.272 & 0.393 & 0.657 & 0.062 & 50.05 \\
\hline
\end{tabular}


Supporting Information (Texts S1-S9, Figures S1-S41 and Tables S1-S12)

\begin{tabular}{|c|c|c|c|c|c|c|c|c|c|c|}
\hline M0936 & 128 & exp05 & 1024bit-ECFP4 & SVM & 0.669 & 0.221 & 0.363 & 0.661 & 0.008 & 50.05 \\
\hline M0937 & 128 & exp06 & 1024bit-ECFP4 & SVM & 0.653 & 0.249 & 0.374 & 0.661 & 0.008 & 50.05 \\
\hline M0938 & 128 & exp07 & 1024bit-ECFP4 & SVM & 0.539 & 0.326 & 0.440 & 0.690 & 0.151 & 50.05 \\
\hline M0939 & 128 & exp08 & 1024bit-ECFP4 & SVM & 0.603 & 0.272 & 0.392 & 0.697 & 0.094 & 50000.00 .05 \\
\hline M0940 & 128 & exp09 & 1024bit-ECFP4 & SVM & 0.591 & 0.283 & 0.400 & 0.641 & 0.050 & 50.05 \\
\hline M0941 & 128 & exp10 & 1024bit-ECFP4 & SVM & 0.622 & 0.265 & 0.396 & 0.654 & 0.032 & 50.05 \\
\hline M0942 & 128 & exp11 & 1024bit-ECFP4 & SVM & 0.671 & 0.228 & 0.354 & 0.634 & 0.037 & 50.05 \\
\hline M0943 & 128 & exp12 & 1024bit-ECFP4 & SVM & 0.606 & 0.263 & 0.401 & 0.631 & 0.025 & 50.05 \\
\hline M0944 & 128 & exp13 & 1024bit-ECFP4 & SVM & 0.683 & 0.227 & 0.363 & 0.634 & 0.049 & 50.05 \\
\hline M0945 & 128 & exp14 & 1024bit-ECFP4 & SVM & 0.584 & 0.293 & 0.415 & 0.671 & 0.087 & 50.1 \\
\hline M0946 & 128 & exp15 & 1024bit-ECFP4 & SVM & 0.684 & 0.227 & 0.346 & 0.637 & 0.047 & 50.05 \\
\hline M0947 & 128 & exp16 & 1024bit-ECFP4 & SVM & 0.592 & 0.275 & 0.385 & 0.655 & 0.063 & 50.05 \\
\hline M0948 & 128 & exp17 & 1024bit-ECFP4 & SVM & 0.598 & 0.287 & 0.401 & 0.632 & 0.034 & 50.05 \\
\hline M0949 & 128 & exp18 & 1024bit-ECFP4 & SVM & 0.603 & 0.272 & 0.395 & 0.637 & 0.034 & 50000.00 .1 \\
\hline M0950 & 128 & exp19 & 1024bit-ECFP4 & SVM & 0.622 & 0.263 & 0.386 & 0.656 & 0.034 & 10.05 \\
\hline M0951 & 128 & exp01 & 1024bit-ECFP4 & GBM & 0.677 & 0.224 & 0.362 & 0.640 & 0.037 & 1000.2 \\
\hline M0952 & 128 & exp02 & 1024bit-ECFP4 & GBM & 0.695 & 0.225 & 0.364 & 0.634 & 0.061 & 4000.1 \\
\hline M0953 & 128 & exp03 & 1024bit-ECFP4 & GBM & 0.616 & 0.262 & 0.384 & 0.652 & 0.036 & 1000.3 \\
\hline M0954 & 128 & exp04 & 1024bit-ECFP4 & GBM & 0.607 & 0.264 & 0.386 & 0.650 & 0.043 & 4000.1 \\
\hline M0955 & 128 & exp05 & 1024bit-ECFP4 & GBM & 0.669 & 0.219 & 0.365 & 0.662 & 0.007 & 3000.1 \\
\hline M0956 & 128 & exp06 & 1024bit-ECFP4 & GBM & 0.596 & 0.287 & 0.403 & 0.660 & 0.064 & 500.3 \\
\hline M0957 & 128 & exp07 & 1024bit-ECFP4 & GBM & 0.574 & 0.300 & 0.421 & 0.660 & 0.086 & 2000.2 \\
\hline M0958 & 128 & exp08 & 1024bit-ECFP4 & GBM & 0.569 & 0.298 & 0.414 & 0.665 & 0.096 & 1000.3 \\
\hline M0959 & 128 & exp09 & 1024bit-ECFP4 & GBM & 0.584 & 0.288 & 0.405 & 0.645 & 0.061 & 1000.3 \\
\hline M0960 & 128 & exp10 & 1024bit-ECFP4 & GBM & 0.649 & 0.246 & 0.386 & 0.652 & 0.003 & 3000.1 \\
\hline M0961 & 128 & exp11 & 1024bit-ECFP4 & GBM & 0.675 & 0.225 & 0.356 & 0.626 & 0.049 & 3000.1 \\
\hline M0962 & 128 & exp12 & 1024bit-ECFP4 & GBM & 0.556 & 0.292 & 0.415 & 0.640 & 0.084 & 1000.1 \\
\hline M0963 & 128 & exp13 & 1024bit-ECFP4 & GBM & 0.695 & 0.217 & 0.354 & 0.647 & 0.048 & 2000.1 \\
\hline M0964 & 128 & exp14 & 1024bit-ECFP4 & GBM & 0.604 & 0.279 & 0.415 & 0.658 & 0.054 & 1000.2 \\
\hline M0965 & 128 & exp15 & 1024bit-ECFP4 & GBM & 0.670 & 0.234 & 0.360 & 0.653 & 0.017 & 1000.2 \\
\hline M0966 & 128 & exp16 & 1024bit-ECFP4 & GBM & 0.567 & 0.290 & 0.404 & 0.674 & 0.107 & 1000.2 \\
\hline M0967 & 128 & exp17 & 1024bit-ECFP4 & GBM & 0.621 & 0.269 & 0.400 & 0.615 & 0.006 & 500.3 \\
\hline M0968 & 128 & exp18 & 1024bit-ECFP4 & GBM & 0.639 & 0.243 & 0.390 & 0.625 & 0.014 & 500.3 \\
\hline M0969 & 128 & exp19 & 1024bit-ECFP4 & GBM & 0.630 & 0.257 & 0.391 & 0.657 & 0.027 & 1000.1 \\
\hline M0970 & 128 & exp01 & 1024bit-ECFP4 & RF & 0.688 & 0.218 & 0.346 & 0.623 & 0.065 & 500 \\
\hline M0971 & 128 & $\exp 02$ & 1024bit-ECFP4 & $\mathrm{RF}$ & 0.682 & 0.234 & 0.369 & 0.619 & 0.063 & 400 \\
\hline M0972 & 128 & exp03 & 1024bit-ECFP4 & $\mathrm{RF}$ & 0.623 & 0.257 & 0.381 & 0.658 & 0.035 & 800 \\
\hline M0973 & 128 & exp04 & 1024bit-ECFP4 & $\mathrm{RF}$ & 0.598 & 0.269 & 0.390 & 0.647 & 0.049 & 100 \\
\hline M0974 & 128 & exp05 & 1024bit-ECFP4 & $\mathrm{RF}$ & 0.642 & 0.239 & 0.365 & 0.662 & 0.020 & 400 \\
\hline M0975 & 128 & exp06 & 1024bit-ECFP4 & $\mathrm{RF}$ & 0.612 & 0.276 & 0.398 & 0.658 & 0.046 & 1000 \\
\hline M0976 & 128 & exp07 & 1024bit-ECFP4 & $\mathrm{RF}$ & 0.560 & 0.304 & 0.418 & 0.657 & 0.097 & 400 \\
\hline M0977 & 128 & exp08 & 1024bit-ECFP4 & $\mathrm{RF}$ & 0.590 & 0.285 & 0.402 & 0.678 & 0.089 & 50 \\
\hline M0978 & 128 & exp09 & 1024bit-ECFP4 & $\mathrm{RF}$ & 0.518 & 0.327 & 0.420 & 0.628 & 0.110 & 1000 \\
\hline M0979 & 128 & exp10 & 1024bit-ECFP4 & $\mathrm{RF}$ & 0.611 & 0.272 & 0.396 & 0.641 & 0.030 & 500 \\
\hline M0980 & 128 & $\exp 11$ & 1024bit-ECFP4 & $\mathrm{RF}$ & 0.635 & 0.252 & 0.366 & 0.609 & 0.026 & 100 \\
\hline
\end{tabular}


Supporting Information (Texts S1-S9, Figures S1-S41 and Tables S1-S12)

\begin{tabular}{|c|c|c|c|c|c|c|c|c|c|c|}
\hline M0981 & 128 & exp12 & 1024bit-ECFP4 & $\mathrm{RF}$ & 0.571 & 0.284 & 0.408 & 0.642 & 0.071 & 900 \\
\hline M0982 & 128 & exp13 & 1024bit-ECFP4 & $\mathrm{RF}$ & 0.704 & 0.217 & 0.363 & 0.633 & 0.071 & 50 \\
\hline M0983 & 128 & exp14 & 1024bit-ECFP4 & $\mathrm{RF}$ & 0.578 & 0.298 & 0.416 & 0.657 & 0.079 & 1000 \\
\hline M0984 & 128 & exp15 & 1024bit-ECFP4 & $\mathrm{RF}$ & 0.665 & 0.234 & 0.350 & 0.612 & 0.053 & 300 \\
\hline M0985 & 128 & exp16 & 1024bit-ECFP4 & $\mathrm{RF}$ & 0.580 & 0.276 & 0.378 & 0.661 & 0.081 & 500 \\
\hline M0986 & 128 & exp17 & 1024bit-ECFP4 & $\mathrm{RF}$ & 0.597 & 0.287 & 0.390 & 0.603 & 0.006 & 1000 \\
\hline M0987 & 128 & exp18 & 1024bit-ECFP4 & $\mathrm{RF}$ & 0.621 & 0.257 & 0.386 & 0.636 & 0.015 & 900 \\
\hline M0988 & 128 & exp19 & 1024bit-ECFP4 & $\mathrm{RF}$ & 0.630 & 0.253 & 0.378 & 0.652 & 0.022 & 800 \\
\hline M0989 & 128 & exp01 & 2048bit-ECFP4 & KNN & 0.682 & 0.227 & 0.363 & 0.622 & 0.060 & 7 distance \\
\hline M0990 & 128 & exp02 & 2048bit-ECFP4 & KNN & 0.651 & 0.263 & 0.384 & 0.639 & 0.012 & 3 distance \\
\hline M0991 & 128 & exp03 & 2048bit-ECFP4 & KNN & 0.634 & 0.247 & 0.375 & 0.637 & 0.003 & 7 distance \\
\hline M0992 & 128 & exp04 & 2048bit-ECFP4 & KNN & 0.586 & 0.278 & 0.399 & 0.668 & 0.082 & 5 distance \\
\hline M0993 & 128 & exp05 & 2048bit-ECFP4 & KNN & 0.652 & 0.239 & 0.365 & 0.659 & 0.007 & 5 uniform \\
\hline M0994 & 128 & exp06 & 2048bit-ECFP4 & KNN & 0.655 & 0.243 & 0.372 & 0.647 & 0.008 & 7 distance \\
\hline M0995 & 128 & exp07 & 2048bit-ECFP4 & KNN & 0.544 & 0.323 & 0.416 & 0.654 & 0.110 & 5 distance \\
\hline M0996 & 128 & exp08 & 2048bit-ECFP4 & KNN & 0.548 & 0.319 & 0.436 & 0.647 & 0.099 & 7 distance \\
\hline M0997 & 128 & exp09 & 2048bit-ECFP4 & KNN & 0.534 & 0.321 & 0.413 & 0.618 & 0.084 & 5 distance \\
\hline M0998 & 128 & $\exp 10$ & 2048bit-ECFP4 & KNN & 0.600 & 0.281 & 0.403 & 0.614 & 0.014 & 5 distance \\
\hline M0999 & 128 & $\exp 11$ & 2048bit-ECFP4 & KNN & 0.618 & 0.270 & 0.387 & 0.617 & 0.001 & 7 distance \\
\hline M1000 & 128 & exp12 & 2048bit-ECFP4 & KNN & 0.593 & 0.268 & 0.403 & 0.618 & 0.025 & 5 distance \\
\hline M1001 & 128 & exp13 & 2048bit-ECFP4 & KNN & 0.697 & 0.223 & 0.366 & 0.623 & 0.074 & 5 distance \\
\hline M1002 & 128 & exp14 & 2048bit-ECFP4 & KNN & 0.576 & 0.310 & 0.422 & 0.688 & 0.112 & 3 distance \\
\hline M1003 & 128 & exp15 & 2048bit-ECFP4 & KNN & 0.651 & 0.246 & 0.356 & 0.637 & 0.014 & 7 distance \\
\hline M1004 & 128 & exp16 & 2048bit-ECFP4 & KNN & 0.576 & 0.283 & 0.401 & 0.654 & 0.078 & 5 distance \\
\hline M1005 & 128 & exp17 & 2048bit-ECFP4 & KNN & 0.585 & 0.302 & 0.400 & 0.620 & 0.035 & 5 uniform \\
\hline M1006 & 128 & exp18 & 2048bit-ECFP4 & KNN & 0.614 & 0.269 & 0.405 & 0.634 & 0.020 & 5 distance \\
\hline M1007 & 128 & exp19 & 2048bit-ECFP4 & KNN & 0.617 & 0.263 & 0.385 & 0.628 & 0.011 & 5 distance \\
\hline M1008 & 128 & exp01 & 2048bit-ECFP4 & SVM & 0.740 & 0.181 & 0.326 & 0.630 & 0.110 & 50.05 \\
\hline M1009 & 128 & exp02 & 2048bit-ECFP4 & SVM & 0.652 & 0.261 & 0.387 & 0.669 & 0.017 & 50.1 \\
\hline M1010 & 128 & exp03 & 2048bit-ECFP4 & SVM & 0.639 & 0.249 & 0.387 & 0.669 & 0.030 & 50.05 \\
\hline M1011 & 128 & exp04 & 2048bit-ECFP4 & SVM & 0.610 & 0.258 & 0.384 & 0.674 & 0.064 & 50.05 \\
\hline M1012 & 128 & exp05 & 2048bit-ECFP4 & SVM & 0.677 & 0.219 & 0.363 & 0.657 & 0.020 & 50.05 \\
\hline M1013 & 128 & exp06 & 2048bit-ECFP4 & SVM & 0.646 & 0.251 & 0.370 & 0.666 & 0.020 & 50.05 \\
\hline M1014 & 128 & exp07 & 2048bit-ECFP4 & SVM & 0.572 & 0.299 & 0.411 & 0.686 & 0.114 & 50.05 \\
\hline M1015 & 128 & exp08 & 2048bit-ECFP4 & SVM & 0.589 & 0.281 & 0.393 & 0.686 & 0.097 & 50.1 \\
\hline M1016 & 128 & exp09 & 2048bit-ECFP4 & SVM & 0.613 & 0.265 & 0.388 & 0.627 & 0.014 & 50.05 \\
\hline M1017 & 128 & $\exp 10$ & 2048bit-ECFP4 & SVM & 0.639 & 0.253 & 0.387 & 0.650 & 0.011 & 50.05 \\
\hline M1018 & 128 & exp11 & 2048bit-ECFP4 & SVM & 0.641 & 0.257 & 0.384 & 0.628 & 0.013 & 100.1 \\
\hline M1019 & 128 & exp12 & 2048bit-ECFP4 & SVM & 0.547 & 0.300 & 0.428 & 0.663 & 0.116 & 500.005 \\
\hline M1020 & 128 & exp13 & 2048bit-ECFP4 & SVM & 0.699 & 0.218 & 0.364 & 0.641 & 0.058 & 50.05 \\
\hline M1021 & 128 & exp14 & 2048bit-ECFP4 & SVM & 0.575 & 0.305 & 0.430 & 0.703 & 0.128 & 50.05 \\
\hline M1022 & 128 & exp15 & 2048bit-ECFP4 & SVM & 0.707 & 0.208 & 0.334 & 0.636 & 0.071 & 50.05 \\
\hline M1023 & 128 & exp16 & 2048bit-ECFP4 & SVM & 0.599 & 0.269 & 0.380 & 0.680 & 0.081 & 50.05 \\
\hline M1024 & 128 & exp17 & 2048bit-ECFP4 & SVM & 0.599 & 0.289 & 0.394 & 0.637 & 0.038 & 100.05 \\
\hline M1025 & 128 & $\exp 18$ & 2048bit-ECFP4 & SVM & 0.633 & 0.250 & 0.384 & 0.644 & 0.011 & 50.1 \\
\hline
\end{tabular}


Supporting Information (Texts S1-S9, Figures S1-S41 and Tables S1-S12)

\begin{tabular}{|c|c|c|c|c|c|c|c|c|c|c|}
\hline M1026 & 128 & $\exp 19$ & 2048bit-ECFP4 & SVM & 0.631 & 0.251 & 0.384 & 0.647 & 0.016 & 50.05 \\
\hline M1027 & 128 & exp01 & 2048bit-ECFP4 & GBM & 0.700 & 0.208 & 0.358 & 0.611 & 0.089 & 2000.1 \\
\hline M1028 & 128 & exp02 & 2048bit-ECFP4 & GBM & 0.713 & 0.209 & 0.350 & 0.643 & 0.070 & 2000.1 \\
\hline M1029 & 128 & exp03 & 2048bit-ECFP4 & GBM & 0.604 & 0.272 & 0.388 & 0.661 & 0.057 & 4000.1 \\
\hline M1030 & 128 & exp04 & 2048bit-ECFP4 & GBM & 0.595 & 0.269 & 0.388 & 0.662 & 0.067 & 1000.3 \\
\hline M1031 & 128 & exp05 & 2048bit-ECFP4 & GBM & 0.656 & 0.228 & 0.368 & 0.669 & 0.013 & 3000.1 \\
\hline M1032 & 128 & exp06 & 2048bit-ECFP4 & GBM & 0.634 & 0.260 & 0.387 & 0.672 & 0.038 & 1000.2 \\
\hline M1033 & 128 & exp07 & 2048bit-ECFP4 & GBM & 0.562 & 0.312 & 0.418 & 0.647 & 0.085 & 2000.2 \\
\hline M1034 & 128 & exp08 & 2048bit-ECFP4 & GBM & 0.536 & 0.321 & 0.428 & 0.688 & 0.152 & 2000.2 \\
\hline M1035 & 128 & exp09 & 2048bit-ECFP4 & GBM & 0.589 & 0.283 & 0.402 & 0.640 & 0.051 & 2000.2 \\
\hline M1036 & 128 & exp10 & 2048bit-ECFP4 & GBM & 0.628 & 0.264 & 0.388 & 0.640 & 0.012 & 2000.2 \\
\hline M1037 & 128 & $\exp 11$ & 2048bit-ECFP4 & GBM & 0.677 & 0.226 & 0.361 & 0.621 & 0.056 & 3000.1 \\
\hline M1038 & 128 & $\exp 12$ & 2048bit-ECFP4 & GBM & 0.545 & 0.303 & 0.422 & 0.661 & 0.116 & 2000.1 \\
\hline M1039 & 128 & exp13 & 2048bit-ECFP4 & GBM & 0.696 & 0.219 & 0.361 & 0.658 & 0.038 & 3000.1 \\
\hline M1040 & 128 & $\exp 14$ & 2048bit-ECFP4 & GBM & 0.586 & 0.302 & 0.431 & 0.690 & 0.104 & 1000.3 \\
\hline M1041 & 128 & exp15 & 2048bit-ECFP4 & GBM & 0.667 & 0.244 & 0.371 & 0.656 & 0.011 & 3000.1 \\
\hline M1042 & 128 & exp16 & 2048bit-ECFP4 & GBM & 0.586 & 0.274 & 0.388 & 0.672 & 0.086 & 500.4 \\
\hline M1043 & 128 & exp17 & 2048bit-ECFP4 & GBM & 0.556 & 0.321 & 0.402 & 0.649 & 0.093 & 3000.1 \\
\hline M1044 & 128 & $\exp 18$ & 2048bit-ECFP4 & GBM & 0.653 & 0.232 & 0.384 & 0.621 & 0.032 & 500.3 \\
\hline M1045 & 128 & exp19 & 2048bit-ECFP4 & GBM & 0.566 & 0.296 & 0.415 & 0.643 & 0.077 & 500.3 \\
\hline M1046 & 128 & exp01 & 2048bit-ECFP4 & $\mathrm{RF}$ & 0.707 & 0.204 & 0.343 & 0.620 & 0.087 & 300 \\
\hline M1047 & 128 & exp02 & 2048bit-ECFP4 & $\mathrm{RF}$ & 0.696 & 0.225 & 0.360 & 0.653 & 0.043 & 200 \\
\hline M1048 & 128 & exp03 & 2048bit-ECFP4 & $\mathrm{RF}$ & 0.641 & 0.247 & 0.377 & 0.661 & 0.020 & 1000 \\
\hline M1049 & 128 & exp04 & 2048bit-ECFP4 & $\mathrm{RF}$ & 0.623 & 0.250 & 0.375 & 0.651 & 0.028 & 900 \\
\hline M1050 & 128 & $\exp 05$ & 2048bit-ECFP4 & $\mathrm{RF}$ & 0.643 & 0.238 & 0.364 & 0.659 & 0.016 & 600 \\
\hline M1051 & 128 & exp06 & 2048bit-ECFP4 & $\mathrm{RF}$ & 0.612 & 0.275 & 0.389 & 0.661 & 0.049 & 300 \\
\hline M1052 & 128 & exp07 & 2048bit-ECFP4 & $\mathrm{RF}$ & 0.552 & 0.310 & 0.413 & 0.675 & 0.123 & 100 \\
\hline M1053 & 128 & exp08 & 2048bit-ECFP4 & $\mathrm{RF}$ & 0.565 & 0.302 & 0.404 & 0.683 & 0.119 & 1000 \\
\hline M1054 & 128 & exp09 & 2048bit-ECFP4 & $\mathrm{RF}$ & 0.521 & 0.325 & 0.415 & 0.642 & 0.121 & 900 \\
\hline M1055 & 128 & $\exp 10$ & 2048bit-ECFP4 & $\mathrm{RF}$ & 0.615 & 0.269 & 0.391 & 0.633 & 0.018 & 700 \\
\hline M1056 & 128 & exp11 & 2048bit-ECFP4 & $\mathrm{RF}$ & 0.657 & 0.237 & 0.366 & 0.623 & 0.034 & 1000 \\
\hline M1057 & 128 & $\exp 12$ & 2048bit-ECFP4 & $\mathrm{RF}$ & 0.592 & 0.269 & 0.394 & 0.645 & 0.053 & 900 \\
\hline M1058 & 128 & exp13 & 2048bit-ECFP4 & $\mathrm{RF}$ & 0.732 & 0.199 & 0.348 & 0.627 & 0.105 & 900 \\
\hline M1059 & 128 & exp14 & 2048bit-ECFP4 & $\mathrm{RF}$ & 0.552 & 0.321 & 0.431 & 0.688 & 0.136 & 700 \\
\hline M1060 & 128 & $\exp 15$ & 2048bit-ECFP4 & $\mathrm{RF}$ & 0.667 & 0.234 & 0.344 & 0.631 & 0.036 & 900 \\
\hline M1061 & 128 & exp16 & 2048bit-ECFP4 & $\mathrm{RF}$ & 0.574 & 0.281 & 0.385 & 0.694 & 0.120 & 800 \\
\hline M1062 & 128 & exp17 & 2048bit-ECFP4 & $\mathrm{RF}$ & 0.606 & 0.280 & 0.385 & 0.627 & 0.021 & 700 \\
\hline M1063 & 128 & $\exp 18$ & 2048bit-ECFP4 & $\mathrm{RF}$ & 0.627 & 0.253 & 0.385 & 0.645 & 0.018 & 900 \\
\hline M1064 & 128 & exp19 & 2048bit-ECFP4 & $\mathrm{RF}$ & 0.634 & 0.251 & 0.378 & 0.658 & 0.024 & 800 \\
\hline M1065 & 128 & exp01 & 1024bit-ECFP6 & KNN & 0.705 & 0.207 & 0.344 & 0.618 & 0.087 & 5 distance \\
\hline M1066 & 128 & exp02 & 1024bit-ECFP6 & KNN & 0.695 & 0.233 & 0.362 & 0.607 & 0.088 & 5 distance \\
\hline M1067 & 128 & exp03 & 1024bit-ECFP6 & KNN & 0.656 & 0.231 & 0.360 & 0.627 & 0.029 & 5 distance \\
\hline M1068 & 128 & exp04 & 1024bit-ECFP6 & KNN & 0.569 & 0.302 & 0.417 & 0.636 & 0.067 & 3 distance \\
\hline M1069 & 128 & $\exp 05$ & 1024bit-ECFP6 & KNN & 0.642 & 0.248 & 0.370 & 0.655 & 0.013 & 5 uniform \\
\hline M1070 & 128 & exp06 & 1024bit-ECFP6 & KNN & 0.619 & 0.271 & 0.397 & 0.647 & 0.028 & 5 distance \\
\hline
\end{tabular}


Supporting Information (Texts S1-S9, Figures S1-S41 and Tables S1-S12)

\begin{tabular}{|c|c|c|c|c|c|c|c|c|c|c|}
\hline M1071 & 128 & exp07 & 1024bit-ECFP6 & KNN & 0.599 & 0.285 & 0.397 & 0.638 & 0.039 & 5 distance \\
\hline M1072 & 128 & exp08 & 1024bit-ECFP6 & KNN & 0.607 & 0.280 & 0.404 & 0.649 & 0.042 & 5 distance \\
\hline M1073 & 128 & exp09 & 1024bit-ECFP6 & KNN & 0.533 & 0.323 & 0.413 & 0.621 & 0.088 & 7 distance \\
\hline M1074 & 128 & exp10 & 1024bit-ECFP6 & KNN & 0.599 & 0.285 & 0.400 & 0.629 & 0.030 & 5 distance \\
\hline M1075 & 128 & exp11 & 1024bit-ECFP6 & KNN & 0.629 & 0.280 & 0.389 & 0.587 & 0.042 & 3 distance \\
\hline M1076 & 128 & $\exp 12$ & 1024bit-ECFP6 & KNN & 0.595 & 0.268 & 0.379 & 0.629 & 0.034 & 5 uniform \\
\hline M1077 & 128 & exp13 & 1024bit-ECFP6 & KNN & 0.726 & 0.208 & 0.345 & 0.642 & 0.084 & 5 distance \\
\hline M1078 & 128 & $\exp 14$ & 1024bit-ECFP6 & KNN & 0.590 & 0.300 & 0.414 & 0.653 & 0.063 & 3 distance \\
\hline M1079 & 128 & exp15 & 1024bit-ECFP6 & KNN & 0.670 & 0.234 & 0.352 & 0.614 & 0.056 & 5 uniform \\
\hline M1080 & 128 & exp16 & 1024bit-ECFP6 & KNN & 0.606 & 0.259 & 0.390 & 0.626 & 0.020 & 5 distance \\
\hline M1081 & 128 & exp17 & 1024bit-ECFP6 & KNN & 0.592 & 0.302 & 0.410 & 0.614 & 0.022 & 3 distance \\
\hline M1082 & 128 & $\exp 18$ & 1024bit-ECFP6 & KNN & 0.612 & 0.273 & 0.402 & 0.625 & 0.013 & 5 distance \\
\hline M1083 & 128 & exp19 & 1024bit-ECFP6 & KNN & 0.564 & 0.301 & 0.413 & 0.644 & 0.080 & 9 distance \\
\hline M1084 & 128 & exp01 & 1024bit-ECFP6 & SVM & 0.715 & 0.199 & 0.337 & 0.678 & 0.037 & 50.05 \\
\hline M1085 & 128 & exp02 & 1024bit-ECFP6 & SVM & 0.671 & 0.246 & 0.377 & 0.663 & 0.008 & 50.05 \\
\hline M1086 & 128 & exp03 & 1024bit-ECFP6 & SVM & 0.623 & 0.262 & 0.388 & 0.660 & 0.037 & 50.05 \\
\hline M1087 & 128 & exp04 & 1024bit-ECFP6 & SVM & 0.618 & 0.253 & 0.391 & 0.657 & 0.039 & 50.05 \\
\hline M1088 & 128 & exp05 & 1024bit-ECFP6 & SVM & 0.669 & 0.228 & 0.358 & 0.644 & 0.025 & 10.05 \\
\hline M1089 & 128 & exp06 & 1024bit-ECFP6 & SVM & 0.649 & 0.251 & 0.378 & 0.669 & 0.020 & 50.05 \\
\hline M1090 & 128 & exp07 & 1024bit-ECFP6 & SVM & 0.559 & 0.306 & 0.424 & 0.696 & 0.137 & 50.05 \\
\hline M1091 & 128 & exp08 & 1024bit-ECFP6 & SVM & 0.614 & 0.265 & 0.396 & 0.710 & 0.096 & 50.05 \\
\hline M1092 & 128 & exp09 & 1024bit-ECFP6 & SVM & 0.581 & 0.284 & 0.404 & 0.652 & 0.072 & 50.05 \\
\hline M1093 & 128 & exp10 & 1024bit-ECFP6 & SVM & 0.595 & 0.283 & 0.406 & 0.647 & 0.052 & 50.1 \\
\hline M1094 & 128 & $\exp 11$ & 1024bit-ECFP6 & SVM & 0.670 & 0.232 & 0.357 & 0.628 & 0.042 & 50.05 \\
\hline M1095 & 128 & $\exp 12$ & 1024bit-ECFP6 & SVM & 0.627 & 0.248 & 0.377 & 0.651 & 0.024 & 50.05 \\
\hline M1096 & 128 & $\exp 13$ & 1024bit-ECFP6 & SVM & 0.728 & 0.196 & 0.346 & 0.632 & 0.096 & 50.05 \\
\hline M1097 & 128 & $\exp 14$ & 1024bit-ECFP6 & SVM & 0.591 & 0.288 & 0.406 & 0.668 & 0.077 & 50.05 \\
\hline M1098 & 128 & exp15 & 1024bit-ECFP6 & SVM & 0.687 & 0.221 & 0.349 & 0.627 & 0.060 & 50.05 \\
\hline M1099 & 128 & exp16 & 1024bit-ECFP6 & SVM & 0.606 & 0.263 & 0.386 & 0.660 & 0.054 & 50.1 \\
\hline M1100 & 128 & $\exp 17$ & 1024bit-ECFP6 & SVM & 0.563 & 0.313 & 0.415 & 0.635 & 0.072 & 10.05 \\
\hline M1101 & 128 & $\exp 18$ & 1024bit-ECFP6 & SVM & 0.644 & 0.241 & 0.375 & 0.632 & 0.012 & 50.05 \\
\hline M1102 & 128 & exp19 & 1024bit-ECFP6 & SVM & 0.664 & 0.229 & 0.356 & 0.674 & 0.010 & 50.05 \\
\hline M1103 & 128 & exp01 & 1024bit-ECFP6 & GBM & 0.683 & 0.222 & 0.359 & 0.666 & 0.017 & 3000.1 \\
\hline M1104 & 128 & exp02 & 1024bit-ECFP6 & GBM & 0.667 & 0.245 & 0.365 & 0.637 & 0.030 & 2000.1 \\
\hline M1105 & 128 & $\operatorname{exp03}$ & 1024bit-ECFP6 & GBM & 0.589 & 0.283 & 0.392 & 0.659 & 0.070 & 2000.1 \\
\hline M1106 & 128 & exp04 & 1024bit-ECFP6 & GBM & 0.607 & 0.262 & 0.382 & 0.634 & 0.027 & 2000.1 \\
\hline M1107 & 128 & $\exp 05$ & 1024bit-ECFP6 & GBM & 0.628 & 0.248 & 0.389 & 0.619 & 0.009 & 500.2 \\
\hline M1108 & 128 & exp06 & 1024bit-ECFP6 & GBM & 0.618 & 0.277 & 0.396 & 0.643 & 0.025 & 500.4 \\
\hline M1109 & 128 & exp07 & 1024bit-ECFP6 & GBM & 0.580 & 0.288 & 0.408 & 0.648 & 0.068 & 2000.1 \\
\hline M1110 & 128 & exp08 & 1024bit-ECFP6 & GBM & 0.560 & 0.301 & 0.414 & 0.676 & 0.116 & 1000.3 \\
\hline M1111 & 128 & exp09 & 1024bit-ECFP6 & GBM & 0.524 & 0.325 & 0.415 & 0.631 & 0.107 & 2000.1 \\
\hline M1112 & 128 & $\exp 10$ & 1024bit-ECFP6 & GBM & 0.563 & 0.311 & 0.421 & 0.613 & 0.050 & 1000.3 \\
\hline M1113 & 128 & $\exp 11$ & 1024bit-ECFP6 & GBM & 0.642 & 0.249 & 0.364 & 0.577 & 0.065 & 2000.1 \\
\hline M1114 & 128 & $\exp 12$ & 1024bit-ECFP6 & GBM & 0.532 & 0.319 & 0.418 & 0.661 & 0.129 & 3000.1 \\
\hline M1115 & 128 & exp13 & 1024bit-ECFP6 & GBM & 0.697 & 0.214 & 0.357 & 0.616 & 0.081 & 3000.1 \\
\hline
\end{tabular}


Supporting Information (Texts S1-S9, Figures S1-S41 and Tables S1-S12)

\begin{tabular}{|c|c|c|c|c|c|c|c|c|c|c|}
\hline M1116 & 128 & exp14 & 1024bit-ECFP6 & GBM & 0.585 & 0.292 & 0.421 & 0.630 & 0.045 & 2000.1 \\
\hline M1117 & 128 & exp15 & 1024bit-ECFP6 & GBM & 0.662 & 0.236 & 0.376 & 0.629 & 0.033 & 2000.1 \\
\hline M1118 & 128 & exp16 & 1024bit-ECFP6 & GBM & 0.608 & 0.260 & 0.380 & 0.645 & 0.037 & 2000.1 \\
\hline M1119 & 128 & exp17 & 1024bit-ECFP6 & GBM & 0.610 & 0.277 & 0.402 & 0.602 & 0.008 & 1000.2 \\
\hline M1120 & 128 & exp18 & 1024bit-ECFP6 & GBM & 0.658 & 0.231 & 0.375 & 0.594 & 0.064 & 1000.2 \\
\hline M1121 & 128 & exp19 & 1024bit-ECFP6 & GBM & 0.604 & 0.270 & 0.385 & 0.652 & 0.048 & 1000.2 \\
\hline M1122 & 128 & exp01 & 1024bit-ECFP6 & $\mathrm{RF}$ & 0.693 & 0.214 & 0.343 & 0.631 & 0.062 & 500 \\
\hline M1123 & 128 & $\exp 02$ & 1024bit-ECFP6 & $\mathrm{RF}$ & 0.677 & 0.239 & 0.366 & 0.621 & 0.056 & 500 \\
\hline M1124 & 128 & exp03 & 1024bit-ECFP6 & $\mathrm{RF}$ & 0.615 & 0.266 & 0.383 & 0.662 & 0.047 & 800 \\
\hline M1125 & 128 & exp04 & 1024bit-ECFP6 & $\mathrm{RF}$ & 0.599 & 0.268 & 0.385 & 0.631 & 0.032 & 300 \\
\hline M1126 & 128 & exp05 & 1024bit-ECFP6 & $\mathrm{RF}$ & 0.649 & 0.235 & 0.358 & 0.631 & 0.018 & 100 \\
\hline M1127 & 128 & exp06 & 1024bit-ECFP6 & $\mathrm{RF}$ & 0.605 & 0.279 & 0.397 & 0.651 & 0.046 & 300 \\
\hline M1128 & 128 & exp07 & 1024bit-ECFP6 & $\mathrm{RF}$ & 0.559 & 0.304 & 0.415 & 0.663 & 0.104 & 400 \\
\hline M1129 & 128 & exp08 & 1024bit-ECFP6 & $\mathrm{RF}$ & 0.607 & 0.269 & 0.383 & 0.674 & 0.067 & 1000 \\
\hline M1130 & 128 & exp09 & 1024bit-ECFP6 & $\mathrm{RF}$ & 0.501 & 0.338 & 0.422 & 0.644 & 0.143 & 100 \\
\hline M1131 & 128 & exp10 & 1024bit-ECFP6 & $\mathrm{RF}$ & 0.599 & 0.281 & 0.399 & 0.629 & 0.030 & 900 \\
\hline M1132 & 128 & exp11 & 1024bit-ECFP6 & $\mathrm{RF}$ & 0.622 & 0.262 & 0.375 & 0.594 & 0.028 & 100 \\
\hline M1133 & 128 & exp12 & 1024bit-ECFP6 & $\mathrm{RF}$ & 0.596 & 0.266 & 0.386 & 0.643 & 0.047 & 100 \\
\hline M1134 & 128 & exp13 & 1024bit-ECFP6 & $\mathrm{RF}$ & 0.736 & 0.199 & 0.343 & 0.624 & 0.112 & 900 \\
\hline M1135 & 128 & exp14 & 1024bit-ECFP6 & $\mathrm{RF}$ & 0.560 & 0.308 & 0.428 & 0.644 & 0.084 & 1000 \\
\hline M1136 & 128 & exp15 & 1024bit-ECFP6 & $\mathrm{RF}$ & 0.664 & 0.232 & 0.357 & 0.596 & 0.068 & 600 \\
\hline M1137 & 128 & exp16 & 1024bit-ECFP6 & $\mathrm{RF}$ & 0.589 & 0.271 & 0.378 & 0.660 & 0.071 & 400 \\
\hline M1138 & 128 & exp17 & 1024bit-ECFP6 & $\mathrm{RF}$ & 0.590 & 0.292 & 0.404 & 0.609 & 0.019 & 500 \\
\hline M1139 & 128 & exp18 & 1024bit-ECFP6 & $\mathrm{RF}$ & 0.623 & 0.254 & 0.387 & 0.616 & 0.007 & 1000 \\
\hline M1140 & 128 & exp19 & 1024bit-ECFP6 & $\mathrm{RF}$ & 0.633 & 0.252 & 0.373 & 0.644 & 0.011 & 800 \\
\hline M1141 & 128 & exp01 & 2048bit-ECFP6 & KNN & 0.652 & 0.246 & 0.366 & 0.603 & 0.049 & 7 distance \\
\hline M1142 & 128 & $\exp 02$ & 2048bit-ECFP6 & KNN & 0.668 & 0.250 & 0.373 & 0.617 & 0.051 & 3 distance \\
\hline M1143 & 128 & exp03 & 2048bit-ECFP6 & KNN & 0.659 & 0.232 & 0.361 & 0.629 & 0.030 & 7 distance \\
\hline M1144 & 128 & exp04 & 2048bit-ECFP6 & KNN & 0.566 & 0.292 & 0.404 & 0.658 & 0.092 & 5 distance \\
\hline M1145 & 128 & exp05 & 2048bit-ECFP6 & KNN & 0.613 & 0.269 & 0.386 & 0.655 & 0.042 & 5 uniform \\
\hline M1146 & 128 & exp06 & 2048bit-ECFP6 & KNN & 0.633 & 0.258 & 0.381 & 0.648 & 0.015 & 7 distance \\
\hline M1147 & 128 & exp07 & 2048bit-ECFP6 & KNN & 0.581 & 0.291 & 0.395 & 0.641 & 0.060 & 5 distance \\
\hline M1148 & 128 & exp08 & 2048bit-ECFP6 & KNN & 0.585 & 0.290 & 0.414 & 0.655 & 0.070 & 5 distance \\
\hline M1149 & 128 & exp09 & 2048bit-ECFP6 & KNN & 0.554 & 0.303 & 0.388 & 0.608 & 0.054 & 7 distance \\
\hline M1150 & 128 & $\exp 10$ & 2048bit-ECFP6 & KNN & 0.649 & 0.250 & 0.388 & 0.643 & 0.006 & 3 uniform \\
\hline M1151 & 128 & exp11 & 2048bit-ECFP6 & KNN & 0.658 & 0.242 & 0.369 & 0.614 & 0.044 & 5 distance \\
\hline M1152 & 128 & exp12 & 2048bit-ECFP6 & KNN & 0.622 & 0.249 & 0.379 & 0.642 & 0.020 & 5 distance \\
\hline M1153 & 128 & exp13 & 2048bit-ECFP6 & KNN & 0.739 & 0.195 & 0.332 & 0.621 & 0.118 & 5 distance \\
\hline M1154 & 128 & exp14 & 2048bit-ECFP6 & KNN & 0.587 & 0.301 & 0.411 & 0.692 & 0.105 & 3 distance \\
\hline M1155 & 128 & exp15 & 2048bit-ECFP6 & KNN & 0.647 & 0.257 & 0.366 & 0.621 & 0.026 & 9 uniform \\
\hline M1156 & 128 & exp16 & 2048bit-ECFP6 & KNN & 0.587 & 0.273 & 0.388 & 0.699 & 0.112 & 5 distance \\
\hline M1157 & 128 & exp17 & 2048bit-ECFP6 & KNN & 0.633 & 0.271 & 0.394 & 0.649 & 0.016 & 3 distance \\
\hline M1158 & 128 & exp18 & 2048bit-ECFP6 & KNN & 0.613 & 0.270 & 0.398 & 0.641 & 0.028 & 5 distance \\
\hline M1159 & 128 & exp19 & 2048bit-ECFP6 & KNN & 0.593 & 0.281 & 0.397 & 0.646 & 0.053 & 9 distance \\
\hline M1160 & 128 & exp01 & 2048bit-ECFP6 & SVM & 0.717 & 0.198 & 0.337 & 0.675 & 0.042 & 50.05 \\
\hline
\end{tabular}


Supporting Information (Texts S1-S9, Figures S1-S41 and Tables S1-S12)

\begin{tabular}{|c|c|c|c|c|c|c|c|c|c|c|}
\hline M1161 & 128 & exp02 & 2048bit-ECFP6 & SVM & 0.658 & 0.255 & 0.382 & 0.675 & 0.017 & 50.05 \\
\hline M1162 & 128 & $\exp 03$ & 2048bit-ECFP6 & SVM & 0.615 & 0.262 & 0.386 & 0.679 & 0.064 & 50.05 \\
\hline M1163 & 128 & exp04 & 2048bit-ECFP6 & SVM & 0.599 & 0.264 & 0.391 & 0.656 & 0.057 & 100.1 \\
\hline M1164 & 128 & exp05 & 2048bit-ECFP6 & SVM & 0.660 & 0.227 & 0.362 & 0.678 & 0.018 & 50.05 \\
\hline M1165 & 128 & exp06 & 2048bit-ECFP6 & SVM & 0.662 & 0.240 & 0.361 & 0.690 & 0.027 & 50.05 \\
\hline M1166 & 128 & exp07 & 2048bit-ECFP6 & SVM & 0.571 & 0.298 & 0.412 & 0.680 & 0.109 & 50.05 \\
\hline M1167 & 128 & exp08 & 2048bit-ECFP6 & SVM & 0.608 & 0.269 & 0.399 & 0.699 & 0.091 & 50.05 \\
\hline M1168 & 128 & exp09 & 2048bit-ECFP6 & SVM & 0.560 & 0.298 & 0.403 & 0.654 & 0.094 & 100.01 \\
\hline M1169 & 128 & exp10 & 2048bit-ECFP6 & SVM & 0.631 & 0.264 & 0.388 & 0.650 & 0.019 & 10.05 \\
\hline M1170 & 128 & exp11 & 2048bit-ECFP6 & SVM & 0.692 & 0.217 & 0.350 & 0.646 & 0.046 & 50.05 \\
\hline M1171 & 128 & exp12 & 2048bit-ECFP6 & SVM & 0.624 & 0.247 & 0.372 & 0.667 & 0.043 & 50.05 \\
\hline M1172 & 128 & exp13 & 2048bit-ECFP6 & SVM & 0.737 & 0.188 & 0.337 & 0.621 & 0.116 & 50.05 \\
\hline M1173 & 128 & $\exp 14$ & 2048bit-ECFP6 & SVM & 0.574 & 0.301 & 0.408 & 0.692 & 0.118 & 50.05 \\
\hline M1174 & 128 & $\exp 15$ & 2048bit-ECFP6 & SVM & 0.684 & 0.230 & 0.362 & 0.652 & 0.032 & 100.05 \\
\hline M1175 & 128 & $\exp 16$ & 2048bit-ECFP6 & SVM & 0.623 & 0.251 & 0.374 & 0.683 & 0.060 & 50.05 \\
\hline M1176 & 128 & exp17 & 2048bit-ECFP6 & SVM & 0.585 & 0.298 & 0.410 & 0.658 & 0.073 & 10.05 \\
\hline M1177 & 128 & exp18 & 2048bit-ECFP6 & SVM & 0.634 & 0.247 & 0.394 & 0.638 & 0.004 & 500.005 \\
\hline M1178 & 128 & exp19 & 2048bit-ECFP6 & SVM & 0.656 & 0.235 & 0.365 & 0.674 & 0.018 & 50.05 \\
\hline M1179 & 128 & exp01 & 2048bit-ECFP6 & GBM & 0.685 & 0.223 & 0.359 & 0.646 & 0.039 & 6000.1 \\
\hline M1180 & 128 & exp02 & 2048bit-ECFP6 & GBM & 0.702 & 0.217 & 0.356 & 0.621 & 0.081 & 2000.1 \\
\hline M1181 & 128 & exp03 & 2048bit-ECFP6 & GBM & 0.602 & 0.273 & 0.387 & 0.684 & 0.082 & 2000.1 \\
\hline M1182 & 128 & exp04 & 2048bit-ECFP6 & GBM & 0.622 & 0.249 & 0.371 & 0.646 & 0.024 & 2000.1 \\
\hline M1183 & 128 & exp05 & 2048bit-ECFP6 & GBM & 0.610 & 0.258 & 0.389 & 0.654 & 0.044 & 2000.1 \\
\hline M1184 & 128 & exp06 & 2048bit-ECFP6 & GBM & 0.663 & 0.238 & 0.363 & 0.663 & 0.000 & 2000.1 \\
\hline M1185 & 128 & exp07 & 2048bit-ECFP6 & GBM & 0.603 & 0.274 & 0.402 & 0.639 & 0.036 & 3000.1 \\
\hline M1186 & 128 & exp08 & 2048bit-ECFP6 & GBM & 0.553 & 0.305 & 0.421 & 0.650 & 0.097 & 3000.1 \\
\hline M1187 & 128 & exp09 & 2048bit-ECFP6 & GBM & 0.577 & 0.286 & 0.386 & 0.638 & 0.061 & 3000.1 \\
\hline M1188 & 128 & exp10 & 2048bit-ECFP6 & GBM & 0.669 & 0.233 & 0.365 & 0.615 & 0.054 & 3000.1 \\
\hline M1189 & 128 & exp11 & 2048bit-ECFP6 & GBM & 0.680 & 0.225 & 0.352 & 0.617 & 0.063 & 2000.1 \\
\hline M1190 & 128 & $\exp 12$ & 2048bit-ECFP6 & GBM & 0.502 & 0.342 & 0.431 & 0.661 & 0.159 & 2000.2 \\
\hline M1191 & 128 & exp13 & 2048bit-ECFP6 & GBM & 0.737 & 0.189 & 0.335 & 0.633 & 0.104 & 1000.3 \\
\hline M1192 & 128 & exp14 & 2048bit-ECFP6 & GBM & 0.598 & 0.284 & 0.417 & 0.674 & 0.076 & 3000.1 \\
\hline M1193 & 128 & $\exp 15$ & 2048bit-ECFP6 & GBM & 0.669 & 0.237 & 0.369 & 0.629 & 0.040 & 3000.1 \\
\hline M1194 & 128 & exp16 & 2048bit-ECFP6 & GBM & 0.603 & 0.261 & 0.374 & 0.689 & 0.086 & 1000.2 \\
\hline M1195 & 128 & exp17 & 2048bit-ECFP6 & GBM & 0.620 & 0.270 & 0.391 & 0.625 & 0.005 & 3000.1 \\
\hline M1196 & 128 & $\exp 18$ & 2048bit-ECFP6 & GBM & 0.641 & 0.243 & 0.380 & 0.611 & 0.030 & 3000.1 \\
\hline M1197 & 128 & exp19 & 2048bit-ECFP6 & GBM & 0.602 & 0.272 & 0.388 & 0.666 & 0.064 & 3000.1 \\
\hline M1198 & 128 & exp01 & 2048bit-ECFP6 & $\mathrm{RF}$ & 0.694 & 0.213 & 0.348 & 0.646 & 0.048 & 300 \\
\hline M1199 & 128 & exp02 & 2048bit-ECFP6 & $\mathrm{RF}$ & 0.685 & 0.234 & 0.362 & 0.638 & 0.047 & 300 \\
\hline M1200 & 128 & exp03 & 2048bit-ECFP6 & $\mathrm{RF}$ & 0.622 & 0.259 & 0.379 & 0.656 & 0.034 & 500 \\
\hline M1201 & 128 & exp04 & 2048bit-ECFP6 & $\mathrm{RF}$ & 0.607 & 0.259 & 0.373 & 0.647 & 0.040 & 900 \\
\hline M1202 & 128 & $\exp 05$ & 2048bit-ECFP6 & $\mathrm{RF}$ & 0.652 & 0.235 & 0.361 & 0.670 & 0.018 & 600 \\
\hline M1203 & 128 & exp06 & 2048bit-ECFP6 & $\mathrm{RF}$ & 0.602 & 0.281 & 0.389 & 0.654 & 0.052 & 500 \\
\hline M1204 & 128 & exp07 & 2048bit-ECFP6 & $\mathrm{RF}$ & 0.560 & 0.303 & 0.401 & 0.666 & 0.106 & 500 \\
\hline M1205 & 128 & exp08 & 2048bit-ECFP6 & $\mathrm{RF}$ & 0.583 & 0.288 & 0.393 & 0.680 & 0.097 & 500 \\
\hline
\end{tabular}


Supporting Information (Texts S1-S9, Figures S1-S41 and Tables S1-S12)

\begin{tabular}{|l|l|l|l|l|l|l|l|l|ll|l|}
\hline M1206 & 128 & exp09 & 2048bit-ECFP6 & RF & 0.505 & 0.336 & 0.412 & 0.640 & 0.135 & 400 \\
\hline M1207 & 128 & exp10 & 2048bit-ECFP6 & RF & 0.638 & 0.253 & 0.374 & 0.637 & 0.001 & 1000 \\
\hline M1208 & 128 & exp11 & 2048bit-ECFP6 & RF & 0.676 & 0.224 & 0.350 & 0.629 & 0.047 & 1000 \\
\hline M1209 & 128 & exp12 & 2048bit-ECFP6 & RF & 0.573 & 0.282 & 0.398 & 0.644 & 0.071 & 500 \\
\hline M1210 & 128 & exp13 & 2048bit-ECFP6 & RF & 0.759 & 0.184 & 0.324 & 0.622 & 0.137 & 900 \\
\hline M1211 & 128 & exp14 & 2048bit-ECFP6 & RF & 0.573 & 0.299 & 0.412 & 0.674 & 0.102 & 400 \\
\hline M1212 & 128 & exp15 & 2048bit-ECFP6 & RF & 0.694 & 0.211 & 0.338 & 0.607 & 0.087 & 900 \\
\hline M1213 & 128 & exp16 & 2048bit-ECFP6 & RF & 0.567 & 0.284 & 0.382 & 0.705 & 0.138 & 1000 \\
\hline M1214 & 128 & exp17 & 2048bit-ECFP6 & RF & 0.625 & 0.268 & 0.383 & 0.637 & 0.012 & 100 \\
\hline M1215 & 128 & exp18 & 2048bit-ECFP6 & RF & 0.623 & 0.254 & 0.384 & 0.621 & 0.002 & 500 \\
\hline M1216 & 128 & exp19 & 2048bit-ECFP6 & RF & 0.645 & 0.243 & 0.366 & 0.666 & 0.021 & 700 & \\
\hline
\end{tabular}

* Optimal parameters for each machine-learning methods as follows

(1) KNN: two parameters are the number of nearest neighbors $(\mathrm{K})$ and weighting schemes (uniform weight or distance-dependent weight) respectively

(2) SVM: two parameters are the penalty parameter $\mathrm{C}$ and kernel parameter gamma respectively

(3) RF: one parameter is the number of decision trees

(4) GBM: two parameters are the number of decision trees and the learning rate 
Table S4. 64 average regression models derived from 1216 individual regression models (Table S3) by averaging over 19 data-splitting schemes.

\begin{tabular}{|c|c|c|c|c|c|c|c|c|}
\hline Model & FS & FP & method & $\mathbf{R}^{2}$ (holdout test set) & MSE(holdout test set) & MAE(holdout test set) & $\mathrm{R}^{2}(\mathrm{CV})$ & $\Delta \mathbf{R}^{2}$ \\
\hline AM01 & full & 1024bit-ECFP4 & KNN & $0.607(0.038)$ & $0.295(0.034)$ & $0.399(0.018)$ & $0.615(0.019)$ & $0.038(0.025)$ \\
\hline AM02 & full & 1024bit-ECFP4 & SVM & $0.638(0.036)$ & $0.252(0.024)$ & $0.379(0.018)$ & $0.647(0.018)$ & $0.038(0.028)$ \\
\hline AM03 & full & 1024bit-ECFP4 & GBM & $0.615(0.046)$ & $0.265(0.028)$ & $0.389(0.019)$ & $0.615(0.018)$ & $0.049(0.024)$ \\
\hline AM04 & full & 1024bit-ECFP4 & $\mathrm{RF}$ & $0.629(0.047)$ & $0.256(0.029)$ & $0.377(0.020)$ & $0.634(0.020)$ & $0.046(0.030)$ \\
\hline AM05 & full & 2048bit-ECFP4 & KNN & $0.610(0.041)$ & $0.292(0.030)$ & $0.398(0.019)$ & $0.616(0.024)$ & $0.041(0.027)$ \\
\hline AM06 & full & 2048bit-ECFP4 & SVM & $0.646(0.036)$ & $0.246(0.025)$ & $0.374(0.018)$ & $0.651(0.018)$ & $0.038(0.028)$ \\
\hline AM07 & full & 2048bit-ECFP4 & GBM & $0.617(0.044)$ & $0.266(0.028)$ & $0.385(0.019)$ & $0.613(0.018)$ & $0.040(0.030)$ \\
\hline AM08 & full & 2048bit-ECFP4 & $\mathrm{RF}$ & $0.635(0.048)$ & $0.252(0.030)$ & $0.372(0.020)$ & $0.641(0.020)$ & $0.047(0.035)$ \\
\hline AM09 & full & 1024bit-ECFP6 & KNN & $0.532(0.051)$ & $0.391(0.054)$ & $0.442(0.028)$ & $0.541(0.032)$ & $0.052(0.035)$ \\
\hline AM10 & full & 1024bit-ECFP6 & SVM & $0.648(0.039)$ & $0.244(0.026)$ & $0.372(0.019)$ & $0.653(0.021)$ & $0.041(0.031)$ \\
\hline AM11 & full & 1024bit-ECFP6 & GBM & $0.615(0.043)$ & $0.265(0.027)$ & $0.389(0.018)$ & $0.602(0.019)$ & $0.048(0.029)$ \\
\hline AM12 & full & 1024bit-ECFP6 & $\mathrm{RF}$ & $0.622(0.051)$ & $0.261(0.032)$ & $0.379(0.021)$ & $0.624(0.021)$ & $0.050(0.033)$ \\
\hline AM13 & full & 2048bit-ECFP6 & KNN & $0.528(0.055)$ & $0.404(0.054)$ & $0.447(0.033)$ & $0.536(0.030)$ & $0.049(0.038)$ \\
\hline AM14 & full & 2048bit-ECFP6 & SVM & $0.653(0.037)$ & $0.240(0.025)$ & $0.368(0.019)$ & $0.659(0.019)$ & $0.039(0.030)$ \\
\hline AM15 & full & 2048bit-ECFP6 & GBM & $0.626(0.044)$ & $0.258(0.027)$ & $0.382(0.020)$ & $0.608(0.023)$ & $0.051(0.032)$ \\
\hline AM16 & full & 2048bit-ECFP6 & RF & $0.635(0.050)$ & $0.253(0.030)$ & $0.372(0.020)$ & $0.632(0.024)$ & $0.051(0.035)$ \\
\hline AM17 & 512 & 1024bit-ECFP4 & KNN & $0.628(0.034)$ & $0.265(0.025)$ & $0.387(0.018)$ & $0.635(0.015)$ & $0.035(0.023)$ \\
\hline AM18 & 512 & 1024bit-ECFP4 & SVM & $0.639(0.039)$ & $0.250(0.025)$ & $0.378(0.019)$ & $0.654(0.019)$ & $0.042(0.029)$ \\
\hline AM19 & 512 & 1024bit-ECFP4 & GBM & $0.620(0.043)$ & $0.263(0.026)$ & $0.386(0.018)$ & $0.626(0.018)$ & $0.046(0.027)$ \\
\hline AM20 & 512 & 1024bit-ECFP4 & $\mathrm{RF}$ & $0.628(0.048)$ & $0.257(0.030)$ & $0.377(0.021)$ & $0.636(0.020)$ & $0.048(0.030)$ \\
\hline AM21 & 512 & 2048bit-ECFP4 & KNN & $0.641(0.038)$ & $0.254(0.025)$ & $0.379(0.019)$ & $0.649(0.018)$ & $0.040(0.026)$ \\
\hline AM22 & 512 & 2048bit-ECFP4 & SVM & $0.649(0.040)$ & $0.243(0.027)$ & $0.373(0.020)$ & $0.661(0.017)$ & $0.042(0.030)$ \\
\hline AM23 & 512 & 2048bit-ECFP4 & GBM & $0.620(0.044)$ & $0.264(0.028)$ & $0.382(0.019)$ & $0.630(0.017)$ & $0.043(0.029)$ \\
\hline AM24 & 512 & 2048bit-ECFP4 & $\mathrm{RF}$ & $0.634(0.048)$ & $0.253(0.030)$ & $0.372(0.020)$ & $0.645(0.020)$ & $0.050(0.034)$ \\
\hline AM25 & 512 & 1024bit-ECFP6 & KNN & $0.603(0.037)$ & $0.296(0.033)$ & $0.398(0.018)$ & $0.610(0.029)$ & $0.037(0.027)$ \\
\hline AM26 & 512 & 1024bit-ECFP6 & SVM & $0.643(0.042)$ & $0.248(0.028)$ & $0.374(0.021)$ & $0.654(0.023)$ & $0.048(0.035)$ \\
\hline AM27 & 512 & 1024bit-ECFP6 & GBM & $0.622(0.044)$ & $0.261(0.028)$ & $0.383(0.018)$ & $0.616(0.025)$ & $0.054(0.026)$ \\
\hline AM28 & 512 & 1024bit-ECFP6 & RF & $0.622(0.051)$ & $0.262(0.032)$ & $0.379(0.021)$ & $0.629(0.021)$ & $0.051(0.033)$ \\
\hline AM29 & 512 & 2048bit-ECFP6 & KNN & $0.631(0.041)$ & $0.259(0.028)$ & $0.380(0.018)$ & $0.640(0.020)$ & $0.045(0.027)$ \\
\hline AM30 & 512 & 2048bit-ECFP6 & SVM & $0.646(0.045)$ & $0.245(0.031)$ & $0.372(0.023)$ & $0.660(0.018)$ & $0.046(0.036)$ \\
\hline AM31 & 512 & 2048bit-ECFP6 & GBM & $0.626(0.050)$ & $0.259(0.032)$ & $0.380(0.024)$ & $0.622(0.023)$ & $0.055(0.031)$ \\
\hline AM32 & 512 & 2048bit-ECFP6 & $\mathrm{RF}$ & $0.635(0.051)$ & $0.253(0.031)$ & $0.372(0.021)$ & $0.640(0.023)$ & $0.052(0.034)$ \\
\hline AM33 & 256 & 1024bit-ECFP4 & KNN & $0.629(0.044)$ & $0.261(0.030)$ & $0.383(0.021)$ & $0.641(0.018)$ & $0.042(0.029)$ \\
\hline AM34 & 256 & 1024bit-ECFP4 & SVM & $0.647(0.042)$ & $0.244(0.028)$ & $0.372(0.020)$ & $0.663(0.021)$ & $0.045(0.033)$ \\
\hline AM35 & 256 & 1024bit-ECFP4 & GBM & $0.623(0.048)$ & $0.261(0.031)$ & $0.385(0.019)$ & $0.643(0.017)$ & $0.051(0.033)$ \\
\hline AM36 & 256 & 1024bit-ECFP4 & $\mathrm{RF}$ & $0.627(0.047)$ & $0.258(0.030)$ & $0.378(0.021)$ & $0.643(0.019)$ & $0.049(0.029)$ \\
\hline AM37 & 256 & 2048bit-ECFP4 & KNN & $0.640(0.047)$ & $0.252(0.031)$ & $0.377(0.022)$ & $0.647(0.020)$ & $0.045(0.029)$ \\
\hline AM38 & 256 & 2048bit-ECFP4 & SVM & $0.644(0.049)$ & $0.246(0.032)$ & $0.376(0.024)$ & $0.665(0.019)$ & $0.051(0.036)$ \\
\hline AM39 & 256 & 2048bit-ECFP4 & GBM & $0.618(0.044)$ & $0.265(0.029)$ & $0.384(0.019)$ & $0.643(0.020)$ & $0.054(0.027)$ \\
\hline
\end{tabular}


Supporting Information (Texts S1-S9, Figures S1-S41 and Tables S1-S12)

\begin{tabular}{|c|c|c|c|c|c|c|c|c|}
\hline AM40 & 256 & 2048bit-ECFP4 & $\mathrm{RF}$ & $0.634(0.050)$ & $0.253(0.032)$ & $0.372(0.021)$ & $0.653(0.021)$ & $0.054(0.035)$ \\
\hline AM41 & 256 & 1024bit-ECFP6 & KNN & $0.630(0.043)$ & $0.261(0.031)$ & $0.382(0.019)$ & $0.645(0.017)$ & $0.044(0.031)$ \\
\hline AM42 & 256 & 1024bit-ECFP6 & SVM & $0.629(0.047)$ & $0.257(0.031)$ & $0.382(0.022)$ & $0.658(0.019)$ & $0.051(0.032)$ \\
\hline AM43 & 256 & 1024bit-ECFP6 & GBM & $0.621(0.046)$ & $0.262(0.030)$ & $0.385(0.022)$ & $0.625(0.019)$ & $0.050(0.028)$ \\
\hline AM44 & 256 & 1024bit-ECFP6 & $\mathrm{RF}$ & $0.618(0.051)$ & $0.264(0.032)$ & $0.382(0.021)$ & $0.633(0.020)$ & $0.052(0.036)$ \\
\hline AM45 & 256 & 2048bit-ECFP6 & KNN & $0.637(0.039)$ & $0.253(0.025)$ & $0.376(0.019)$ & $0.655(0.020)$ & $0.046(0.022)$ \\
\hline AM46 & 256 & 2048bit-ECFP6 & SVM & $0.633(0.045)$ & $0.253(0.029)$ & $0.378(0.021)$ & $0.663(0.016)$ & $0.052(0.034)$ \\
\hline AM47 & 256 & 2048bit-ECFP6 & GBM & $0.618(0.052)$ & $0.265(0.033)$ & $0.385(0.023)$ & $0.634(0.018)$ & $0.055(0.031)$ \\
\hline AM48 & 256 & 2048bit-ECFP6 & $\mathrm{RF}$ & $0.628(0.053)$ & $0.257(0.033)$ & $0.374(0.022)$ & $0.645(0.023)$ & $0.056(0.037)$ \\
\hline AM49 & 128 & 1024bit-ECFP4 & KNN & $0.596(0.045)$ & $0.284(0.029)$ & $0.399(0.018)$ & $0.626(0.015)$ & $0.049(0.029)$ \\
\hline AM50 & 128 & 1024bit-ECFP4 & SVM & $0.628(0.043)$ & $0.260(0.027)$ & $0.385(0.022)$ & $0.652(0.019)$ & $0.048(0.035)$ \\
\hline AM51 & 128 & 1024bit-ECFP4 & GBM & $0.626(0.044)$ & $0.259(0.028)$ & $0.388(0.022)$ & $0.648(0.015)$ & $0.047(0.030)$ \\
\hline AM52 & 128 & 1024bit-ECFP4 & $\mathrm{RF}$ & $0.616(0.046)$ & $0.265(0.029)$ & $0.385(0.022)$ & $0.641(0.020)$ & $0.054(0.029)$ \\
\hline AM53 & 128 & 2048bit-ECFP4 & KNN & $0.611(0.045)$ & $0.272(0.030)$ & $0.392(0.022)$ & $0.638(0.020)$ & $0.045(0.039)$ \\
\hline AM54 & 128 & 2048bit-ECFP4 & SVM & $0.632(0.048)$ & $0.256(0.032)$ & $0.383(0.025)$ & $0.658(0.022)$ & $0.054(0.041)$ \\
\hline AM55 & 128 & 2048bit-ECFP4 & GBM & $0.618(0.054)$ & $0.265(0.037)$ & $0.390(0.024)$ & $0.653(0.021)$ & $0.066(0.037)$ \\
\hline AM56 & 128 & 2048bit-ECFP4 & $\mathrm{RF}$ & $0.622(0.054)$ & $0.262(0.035)$ & $0.381(0.024)$ & $0.651(0.022)$ & $0.062(0.043)$ \\
\hline AM57 & 128 & 1024bit-ECFP6 & KNN & $0.621(0.049)$ & $0.268(0.032)$ & $0.387(0.024)$ & $0.630(0.017)$ & $0.049(0.026)$ \\
\hline AM58 & 128 & 1024bit-ECFP6 & SVM & $0.635(0.047)$ & $0.253(0.032)$ & $0.380(0.024)$ & $0.657(0.022)$ & $0.051(0.033)$ \\
\hline AM59 & 128 & 1024bit-ECFP6 & GBM & $0.611(0.047)$ & $0.269(0.032)$ & $0.390(0.021)$ & $0.633(0.025)$ & $0.054(0.034)$ \\
\hline AM60 & 128 & 1024bit-ECFP6 & $\mathrm{RF}$ & $0.617(0.051)$ & $0.265(0.032)$ & $0.383(0.023)$ & $0.635(0.022)$ & $0.055(0.035)$ \\
\hline AM61 & 128 & 2048bit-ECFP6 & KNN & $0.623(0.043)$ & $0.264(0.026)$ & $0.384(0.019)$ & $0.641(0.025)$ & $0.052(0.033)$ \\
\hline AM62 & 128 & 2048bit-ECFP6 & SVM & $0.636(0.048)$ & $0.252(0.032)$ & $0.379(0.023)$ & $0.667(0.020)$ & $0.055(0.035)$ \\
\hline AM63 & 128 & 2048bit-ECFP6 & GBM & $0.628(0.054)$ & $0.257(0.034)$ & $0.381(0.024)$ & $0.645(0.023)$ & $0.060(0.037)$ \\
\hline AM64 & 128 & 2048bit-ECFP6 & $\mathrm{RF}$ & $0.625(0.058)$ & $0.258(0.036)$ & $0.375(0.023)$ & $0.649(0.023)$ & $0.063(0.045)$ \\
\hline
\end{tabular}


Table S5. Evaluation metrics for Dataset-CV with the randomly shuffled $\operatorname{LogHD}_{50}$ values in Y-randomization test of 1216 regression models

\begin{tabular}{|l|c|c|c|c|c|}
\hline Exp & $\mathbf{N}_{\text {unshuffled }}$ & $\mathbf{N}_{\text {shuffled }}$ & $\mathbf{R}^{2}$ & MSE & MAE \\
\hline exp01 & 2 & 642 & 0.001 & 1.294 & 0.891 \\
\hline exp02 & 2 & 642 & 0.002 & 1.268 & 0.882 \\
\hline exp03 & 3 & 641 & 0.000 & 1.347 & 0.903 \\
\hline exp04 & 2 & 642 & 0.000 & 1.335 & 0.889 \\
\hline exp05 & 7 & 637 & 0.003 & 1.285 & 0.873 \\
\hline exp06 & 6 & 638 & 0.001 & 1.298 & 0.886 \\
\hline exp07 & 3 & 641 & 0.004 & 1.251 & 0.861 \\
\hline $\operatorname{exp08}$ & 0 & 644 & 0.000 & 1.357 & 0.879 \\
\hline $\operatorname{exp09}$ & 5 & 639 & 0.001 & 1.298 & 0.882 \\
\hline $\operatorname{exp10}$ & 1 & 643 & 0.001 & 1.304 & 0.879 \\
\hline $\operatorname{exp11}$ & 5 & 639 & 0.001 & 1.384 & 0.905 \\
\hline $\operatorname{exp12}$ & 3 & 641 & 0.001 & 1.313 & 0.888 \\
\hline $\operatorname{exp13}$ & 4 & 640 & 0.002 & 1.285 & 0.868 \\
\hline $\operatorname{exp14}$ & 4 & 640 & 0.000 & 1.348 & 0.896 \\
\hline $\operatorname{exp15}$ & 3 & 641 & 0.000 & 1.339 & 0.890 \\
\hline $\operatorname{exp16}$ & 3 & 641 & 0.000 & 1.380 & 0.908 \\
\hline $\operatorname{exp17}$ & 1 & 643 & 0.000 & 1.339 & 0.884 \\
\hline $\operatorname{exp18}$ & 0 & 644 & 0.001 & 1.303 & 0.903 \\
\hline $\operatorname{exp19}$ & 3 & 641 & 0.005 & 1.437 & 0.925 \\
\hline
\end{tabular}

Notes: (1) "Exp" refers to the random data-splitting scheme; (2) $\mathrm{N}_{\text {unshuffled }}$ is the number of samples in Dataset-CV that is not shuffled. (3) $\mathrm{N}_{\text {shuffled }}$ is the number of samples in Dataset-CV that is randomly shuffled. 
Table S6 1216 individual regression models after applying Y-randomization test

\begin{tabular}{|c|c|c|c|c|c|c|c|c|c|c|}
\hline Model & FS & EXP & FP & Method & $\begin{array}{c}\mathbf{R}^{2} \\
\text { (holdout } \\
\text { test) }\end{array}$ & $\begin{array}{c}\begin{array}{c}\text { MSE } \\
\text { (holdout } \\
\text { test) }\end{array} \\
\end{array}$ & $\begin{array}{c}\text { MAE } \\
\text { (holdout } \\
\text { test) }\end{array}$ & $\begin{array}{c}\mathbf{R}^{2} \\
(\mathbf{C V})\end{array}$ & $\Delta \mathbf{R}^{2}$ & $\begin{array}{l}\text { Parameters from } \\
\text { CV* }^{*}\end{array}$ \\
\hline M0001 & full & exp01 & 1024bit-ECFP4 & KNN & 0.041 & 0.754 & 0.660 & 0.022 & 0.019 & 3 distance \\
\hline M0002 & full & $\exp 02$ & 1024bit-ECFP4 & KNN & 0.001 & 0.828 & 0.691 & 0.014 & 0.013 & 7 distance \\
\hline M0003 & full & $\exp 03$ & 1024bit-ECFP4 & KNN & 0.038 & 0.684 & 0.648 & 0.008 & 0.030 & 3 uniform \\
\hline M0004 & full & $\exp 04$ & 1024bit-ECFP4 & KNN & 0.000 & 1.130 & 0.801 & 0.016 & 0.016 & 1 uniform \\
\hline M0005 & full & exp05 & 1024bit-ECFP4 & KNN & 0.022 & 0.714 & 0.647 & 0.035 & 0.013 & 15 distance \\
\hline M0006 & full & exp06 & 1024bit-ECFP4 & KNN & 0.004 & 0.796 & 0.673 & 0.023 & 0.019 & 9 distance \\
\hline M0007 & full & $\exp 07$ & 1024bit-ECFP4 & KNN & 0.005 & 0.801 & 0.685 & 0.012 & 0.007 & 3 uniform \\
\hline M0008 & full & $\exp 08$ & 1024bit-ECFP4 & KNN & 0.010 & 0.910 & 0.747 & 0.013 & 0.003 & 7 distance \\
\hline M0009 & full & exp09 & 1024bit-ECFP4 & KNN & 0.034 & 0.737 & 0.660 & 0.027 & 0.007 & 3 distance \\
\hline M0010 & full & $\exp 10$ & 1024bit-ECFP4 & KNN & 0.003 & 0.791 & 0.664 & 0.027 & 0.024 & 9 uniform \\
\hline M0011 & full & $\exp 11$ & 1024bit-ECFP4 & KNN & 0.019 & 0.794 & 0.658 & 0.012 & 0.007 & 7 uniform \\
\hline M0012 & full & exp12 & 1024bit-ECFP4 & KNN & 0.000 & 0.773 & 0.677 & 0.009 & 0.009 & 15 distance \\
\hline M0013 & full & $\exp 13$ & 1024bit-ECFP4 & KNN & 0.014 & 0.692 & 0.614 & 0.008 & 0.006 & 13 uniform \\
\hline M0014 & full & exp14 & 1024bit-ECFP4 & KNN & 0.063 & 0.659 & 0.598 & 0.027 & 0.036 & 5 uniform \\
\hline M0015 & full & exp15 & 1024bit-ECFP4 & KNN & 0.002 & 0.762 & 0.680 & 0.008 & 0.006 & 5 uniform \\
\hline M0016 & full & $\exp 16$ & 1024bit-ECFP4 & KNN & 0.015 & 0.752 & 0.654 & 0.031 & 0.016 & 3 distance \\
\hline M0017 & full & $\exp 17$ & 1024bit-ECFP4 & $\mathrm{KNN}$ & 0.004 & 0.936 & 0.755 & 0.031 & 0.027 & 3 distance \\
\hline M0018 & full & $\exp 18$ & 1024bit-ECFP4 & KNN & 0.004 & 0.691 & 0.625 & 0.011 & 0.007 & 11 uniform \\
\hline M0019 & full & $\exp 19$ & 1024bit-ECFP4 & $\mathrm{KNN}$ & 0.003 & 1.558 & 0.928 & 0.015 & 0.012 & 1 uniform \\
\hline M0020 & full & $\exp 01$ & 1024bit-ECFP4 & SVM & 0.021 & 0.691 & 0.626 & 0.014 & 0.007 & 10.1 \\
\hline M0021 & full & $\exp 02$ & 1024bit-ECFP4 & SVM & 0.004 & 0.864 & 0.698 & 0.012 & 0.008 & 10000.00 .1 \\
\hline M0022 & full & exp03 & 1024bit-ECFP4 & SVM & 0.008 & 1.450 & 0.923 & 0.015 & 0.007 & 100000.00 .0005 \\
\hline M0023 & full & $\exp 04$ & 1024bit-ECFP4 & SVM & 0.001 & 0.773 & 0.676 & 0.012 & 0.011 & 50.1 \\
\hline M0024 & full & $\exp 05$ & 1024bit-ECFP4 & SVM & 0.004 & 0.831 & 0.721 & 0.022 & 0.018 & 50.05 \\
\hline M0025 & full & exp06 & 1024bit-ECFP4 & SVM & 0.000 & 0.772 & 0.667 & 0.019 & 0.019 & 10.1 \\
\hline M0026 & full & $\exp 07$ & 1024bit-ECFP4 & SVM & 0.000 & 1.687 & 0.995 & 0.021 & 0.021 & $100000.05 \mathrm{e}-05$ \\
\hline M0027 & full & $\exp 08$ & 1024bit-ECFP4 & SVM & 0.034 & 0.707 & 0.639 & 0.017 & 0.018 & $505 \mathrm{e}-05$ \\
\hline M0028 & full & exp09 & 1024bit-ECFP4 & SVM & 0.022 & 0.728 & 0.666 & 0.030 & 0.008 & 1000.00 .1 \\
\hline M0029 & full & exp10 & 1024bit-ECFP4 & SVM & 0.003 & 1.162 & 0.847 & 0.036 & 0.033 & $5000.05 \mathrm{e}-05$ \\
\hline M0030 & full & exp11 & 1024bit-ECFP4 & SVM & 0.048 & 1.330 & 0.917 & 0.016 & 0.032 & $50000.01 \mathrm{e}-05$ \\
\hline M0031 & full & $\exp 12$ & 1024bit-ECFP4 & SVM & 0.003 & 0.768 & 0.669 & 0.020 & 0.017 & 50.005 \\
\hline M0032 & full & $\exp 13$ & 1024bit-ECFP4 & SVM & 0.006 & 1.319 & 0.905 & 0.011 & 0.005 & 10000.00 .0001 \\
\hline M0033 & full & exp14 & 1024bit-ECFP4 & SVM & 0.019 & 0.705 & 0.619 & 0.024 & 0.005 & 50.005 \\
\hline M0034 & full & exp15 & 1024bit-ECFP4 & SVM & 0.209 & 0.691 & 0.616 & 0.007 & 0.202 & $11 \mathrm{e}-05$ \\
\hline M0035 & full & $\exp 16$ & 1024bit-ECFP4 & SVM & 0.017 & 0.740 & 0.654 & 0.037 & 0.020 & 1000.05 \\
\hline M0036 & full & exp17 & 1024bit-ECFP4 & SVM & 0.001 & 1.044 & 0.808 & 0.026 & 0.025 & 50000.00 .01 \\
\hline M0037 & full & $\exp 18$ & 1024bit-ECFP4 & SVM & 0.001 & 1.006 & 0.805 & 0.015 & 0.014 & $5000.05 \mathrm{e}-05$ \\
\hline
\end{tabular}


Supporting Information (Texts S1-S9, Figures S1-S41 and Tables S1-S12)

\begin{tabular}{|c|c|c|c|c|c|c|c|c|c|c|}
\hline M0038 & full & $\exp 19$ & 1024bit-ECFP4 & SVM & 0.011 & 1.508 & 0.941 & 0.015 & 0.004 & $50000.01 \mathrm{e}-05$ \\
\hline M0039 & full & exp01 & 1024bit-ECFP4 & GBM & 0.014 & 0.686 & 0.613 & 0.013 & 0.001 & 100.2 \\
\hline M0040 & full & exp02 & 1024bit-ECFP4 & GBM & 0.016 & 0.956 & 0.741 & 0.013 & 0.003 & 500.6 \\
\hline M0041 & full & exp03 & 1024bit-ECFP4 & GBM & 0.045 & 0.679 & 0.631 & 0.013 & 0.032 & 500.6 \\
\hline M0042 & full & exp04 & 1024bit-ECFP4 & GBM & 0.005 & 0.880 & 0.718 & 0.015 & 0.010 & 1000.6 \\
\hline M0043 & full & exp05 & 1024bit-ECFP4 & GBM & 0.001 & 0.936 & 0.758 & 0.023 & 0.022 & 2000.4 \\
\hline M0044 & full & exp06 & 1024bit-ECFP4 & GBM & 0.014 & 1.116 & 0.811 & 0.019 & 0.005 & 10000.4 \\
\hline M0045 & full & exp07 & 1024bit-ECFP4 & GBM & 0.003 & 0.878 & 0.719 & 0.009 & 0.006 & 1000.6 \\
\hline M0046 & full & exp08 & 1024bit-ECFP4 & GBM & 0.002 & 0.697 & 0.631 & 0.022 & 0.020 & 100.3 \\
\hline M0047 & full & exp09 & 1024bit-ECFP4 & GBM & 0.023 & 0.881 & 0.716 & 0.027 & 0.004 & 1000.9 \\
\hline M0048 & full & exp10 & 1024bit-ECFP4 & GBM & 0.001 & 0.966 & 0.751 & 0.040 & 0.038 & 7000.1 \\
\hline M0049 & full & $\exp 11$ & 1024bit-ECFP4 & GBM & 0.040 & 0.909 & 0.704 & 0.013 & 0.027 & 500.4 \\
\hline M0050 & full & $\exp 12$ & 1024bit-ECFP4 & GBM & 0.022 & 0.937 & 0.739 & 0.017 & 0.005 & 1000.4 \\
\hline M0051 & full & exp13 & 1024bit-ECFP4 & GBM & 0.000 & 0.708 & 0.628 & 0.015 & 0.015 & 100.3 \\
\hline M0052 & full & $\exp 14$ & 1024bit-ECFP4 & GBM & 0.031 & 0.845 & 0.717 & 0.011 & 0.020 & 2000.6 \\
\hline M0053 & full & exp15 & 1024bit-ECFP4 & GBM & 0.008 & 0.715 & 0.633 & 0.014 & 0.006 & 100.1 \\
\hline M0054 & full & exp16 & 1024bit-ECFP4 & GBM & 0.003 & 0.984 & 0.753 & 0.020 & 0.017 & 10000.6 \\
\hline M0055 & full & $\exp 17$ & 1024bit-ECFP4 & GBM & 0.003 & 1.122 & 0.825 & 0.017 & 0.014 & 9000.3 \\
\hline M0056 & full & $\exp 18$ & 1024bit-ECFP4 & GBM & 0.031 & 0.651 & 0.620 & 0.013 & 0.018 & 100.5 \\
\hline M0057 & full & exp19 & 1024bit-ECFP4 & GBM & 0.019 & 1.433 & 0.877 & 0.015 & 0.004 & 2000.9 \\
\hline M0058 & full & exp01 & 1024bit-ECFP4 & $\mathrm{RF}$ & 0.045 & 0.699 & 0.625 & 0.008 & 0.037 & 600 \\
\hline M0059 & full & exp02 & 1024bit-ECFP4 & $\mathrm{RF}$ & 0.001 & 0.825 & 0.696 & 0.010 & 0.009 & 10 \\
\hline M0060 & full & exp03 & 1024bit-ECFP4 & $\mathrm{RF}$ & 0.041 & 0.683 & 0.649 & 0.005 & 0.036 & 10 \\
\hline M0061 & full & exp04 & 1024bit-ECFP4 & $\mathrm{RF}$ & 0.009 & 0.735 & 0.665 & 0.015 & 0.006 & 100 \\
\hline M0062 & full & $\exp 05$ & 1024bit-ECFP4 & $\mathrm{RF}$ & 0.007 & 0.754 & 0.686 & 0.021 & 0.014 & 300 \\
\hline M0063 & full & exp06 & 1024bit-ECFP4 & $\mathrm{RF}$ & 0.000 & 0.880 & 0.724 & 0.011 & 0.011 & 10 \\
\hline M0064 & full & exp07 & 1024bit-ECFP4 & $\mathrm{RF}$ & 0.010 & 0.769 & 0.672 & 0.006 & 0.004 & 1000 \\
\hline M0065 & full & exp08 & 1024bit-ECFP4 & $\mathrm{RF}$ & 0.003 & 0.835 & 0.703 & 0.009 & 0.006 & 10 \\
\hline M0066 & full & exp09 & 1024bit-ECFP4 & $\mathrm{RF}$ & 0.037 & 0.680 & 0.622 & 0.035 & 0.002 & 900 \\
\hline M0067 & full & $\exp 10$ & 1024bit-ECFP4 & $\mathrm{RF}$ & 0.005 & 0.870 & 0.716 & 0.026 & 0.021 & 700 \\
\hline M0068 & full & exp11 & 1024bit-ECFP4 & $\mathrm{RF}$ & 0.030 & 0.994 & 0.759 & 0.003 & 0.027 & 10 \\
\hline M0069 & full & $\exp 12$ & 1024bit-ECFP4 & $\mathrm{RF}$ & 0.011 & 0.848 & 0.717 & 0.011 & 0.000 & 200 \\
\hline M0070 & full & exp13 & 1024bit-ECFP4 & $\mathrm{RF}$ & 0.002 & 0.817 & 0.698 & 0.009 & 0.007 & 10 \\
\hline M0071 & full & exp14 & 1024bit-ECFP4 & $\mathrm{RF}$ & 0.039 & 0.702 & 0.630 & 0.010 & 0.029 & 100 \\
\hline M0072 & full & $\exp 15$ & 1024bit-ECFP4 & $\mathrm{RF}$ & 0.004 & 0.865 & 0.720 & 0.005 & 0.001 & 500 \\
\hline M0073 & full & $\exp 16$ & 1024bit-ECFP4 & $\mathrm{RF}$ & 0.001 & 0.747 & 0.654 & 0.029 & 0.028 & 100 \\
\hline M0074 & full & $\exp 17$ & 1024bit-ECFP4 & $\mathrm{RF}$ & 0.000 & 0.857 & 0.710 & 0.014 & 0.014 & 200 \\
\hline M0075 & full & $\exp 18$ & 1024bit-ECFP4 & $\mathrm{RF}$ & 0.007 & 0.751 & 0.677 & 0.003 & 0.004 & 10 \\
\hline M0076 & full & exp19 & 1024bit-ECFP4 & $\mathrm{RF}$ & 0.031 & 1.054 & 0.765 & 0.011 & 0.020 & 10 \\
\hline M0077 & full & exp01 & 2048bit-ECFP4 & KNN & 0.031 & 0.732 & 0.638 & 0.019 & 0.012 & 5 distance \\
\hline M0078 & full & exp02 & 2048bit-ECFP4 & KNN & 0.001 & 0.823 & 0.694 & 0.012 & 0.011 & 9 distance \\
\hline M0079 & full & exp03 & 2048bit-ECFP4 & KNN & 0.046 & 0.677 & 0.646 & 0.009 & 0.037 & 3 uniform \\
\hline M0080 & full & exp04 & 2048bit-ECFP4 & KNN & 0.003 & 1.185 & 0.830 & 0.018 & 0.015 & 1 uniform \\
\hline M0081 & full & $\exp 05$ & 2048bit-ECFP4 & KNN & 0.016 & 0.753 & 0.671 & 0.036 & 0.020 & 7 distance \\
\hline M0082 & full & exp06 & 2048bit-ECFP4 & KNN & 0.005 & 0.798 & 0.675 & 0.028 & 0.023 & 9 distance \\
\hline
\end{tabular}


Supporting Information (Texts S1-S9, Figures S1-S41 and Tables S1-S12)

\begin{tabular}{|c|c|c|c|c|c|c|c|c|c|c|}
\hline M0083 & full & exp07 & 2048bit-ECFP4 & KNN & 0.001 & 0.809 & 0.686 & 0.015 & 0.014 & 3 uniform \\
\hline M0084 & full & exp08 & 2048bit-ECFP4 & KNN & 0.013 & 0.919 & 0.754 & 0.012 & 0.001 & 7 distance \\
\hline M0085 & full & exp09 & 2048bit-ECFP4 & KNN & 0.020 & 0.728 & 0.656 & 0.029 & 0.009 & 3 uniform \\
\hline M0086 & full & exp10 & 2048bit-ECFP4 & KNN & 0.007 & 1.172 & 0.835 & 0.023 & 0.016 & 1 uniform \\
\hline M0087 & full & exp11 & 2048bit-ECFP4 & KNN & 0.025 & 0.786 & 0.653 & 0.013 & 0.012 & 11 uniform \\
\hline M0088 & full & $\exp 12$ & 2048bit-ECFP4 & KNN & 0.000 & 0.775 & 0.676 & 0.011 & 0.011 & 15 distance \\
\hline M0089 & full & exp13 & 2048bit-ECFP4 & KNN & 0.033 & 0.679 & 0.618 & 0.009 & 0.024 & 9 uniform \\
\hline M0090 & full & $\exp 14$ & 2048bit-ECFP4 & KNN & 0.019 & 0.986 & 0.774 & 0.030 & 0.011 & 1 uniform \\
\hline M0091 & full & exp15 & 2048bit-ECFP4 & KNN & 0.003 & 0.791 & 0.693 & 0.009 & 0.006 & 3 uniform \\
\hline M0092 & full & exp16 & 2048bit-ECFP4 & KNN & 0.013 & 0.731 & 0.653 & 0.027 & 0.014 & 5 distance \\
\hline M0093 & full & exp17 & 2048bit-ECFP4 & KNN & 0.002 & 0.900 & 0.740 & 0.030 & 0.028 & 5 distance \\
\hline M0094 & full & $\exp 18$ & 2048bit-ECFP4 & KNN & 0.006 & 0.679 & 0.622 & 0.012 & 0.006 & 15 uniform \\
\hline M0095 & full & exp19 & 2048bit-ECFP4 & KNN & 0.020 & 0.873 & 0.691 & 0.011 & 0.009 & 5 uniform \\
\hline M0096 & full & exp01 & 2048bit-ECFP4 & SVM & 0.020 & 0.692 & 0.625 & 0.013 & 0.007 & 10.1 \\
\hline M0097 & full & exp02 & 2048bit-ECFP4 & SVM & 0.002 & 0.898 & 0.707 & 0.012 & 0.010 & 1000.00 .05 \\
\hline M0098 & full & exp03 & 2048bit-ECFP4 & SVM & 0.012 & 1.343 & 0.877 & 0.016 & 0.004 & 100000.00 .0001 \\
\hline M0099 & full & exp04 & 2048bit-ECFP4 & SVM & 0.002 & 1.457 & 0.948 & 0.014 & 0.012 & 50000.00 .0001 \\
\hline M0100 & full & $\exp 05$ & 2048bit-ECFP4 & SVM & 0.001 & 0.902 & 0.749 & 0.024 & 0.022 & 100.01 \\
\hline M0101 & full & exp06 & 2048bit-ECFP4 & SVM & 0.000 & 0.773 & 0.668 & 0.020 & 0.020 & 10.1 \\
\hline M0102 & full & exp07 & 2048bit-ECFP4 & SVM & 0.000 & 1.133 & 0.830 & 0.016 & 0.016 & $10000.05 \mathrm{e}-05$ \\
\hline M0103 & full & exp08 & 2048bit-ECFP4 & SVM & 0.022 & 0.727 & 0.650 & 0.016 & 0.006 & $5001 \mathrm{e}-05$ \\
\hline M0104 & full & exp09 & 2048bit-ECFP4 & SVM & 0.022 & 0.726 & 0.665 & 0.031 & 0.009 & 5000.00 .1 \\
\hline M0105 & full & exp10 & 2048bit-ECFP4 & SVM & 0.033 & 0.803 & 0.658 & 0.026 & 0.007 & $5001 \mathrm{e}-05$ \\
\hline M0106 & full & exp11 & 2048bit-ECFP4 & SVM & 0.014 & 0.983 & 0.768 & 0.018 & 0.004 & $10000.01 \mathrm{e}-05$ \\
\hline M0107 & full & $\exp 12$ & 2048bit-ECFP4 & SVM & 0.003 & 0.832 & 0.713 & 0.024 & 0.021 & 500.001 \\
\hline M0108 & full & $\exp 13$ & 2048bit-ECFP4 & SVM & 0.006 & 1.172 & 0.849 & 0.009 & 0.003 & 1000.00 .0005 \\
\hline M0109 & full & $\exp 14$ & 2048bit-ECFP4 & SVM & 0.025 & 0.698 & 0.613 & 0.028 & 0.003 & 50.005 \\
\hline M0110 & full & exp15 & 2048bit-ECFP4 & SVM & 0.186 & 0.691 & 0.616 & 0.007 & 0.179 & $11 \mathrm{e}-05$ \\
\hline M0111 & full & exp16 & 2048bit-ECFP4 & SVM & 0.017 & 0.726 & 0.644 & 0.037 & 0.020 & 100.05 \\
\hline M0112 & full & $\exp 17$ & 2048bit-ECFP4 & SVM & 0.002 & 1.055 & 0.812 & 0.029 & 0.027 & 5000.005 \\
\hline M0113 & full & $\exp 18$ & 2048bit-ECFP4 & SVM & 0.129 & 0.666 & 0.610 & 0.007 & 0.122 & $11 \mathrm{e}-05$ \\
\hline M0114 & full & exp19 & 2048bit-ECFP4 & SVM & 0.002 & 1.403 & 0.911 & 0.010 & 0.008 & $100000.01 \mathrm{e}-05$ \\
\hline M0115 & full & exp01 & 2048bit-ECFP4 & GBM & 0.011 & 0.686 & 0.614 & 0.012 & 0.001 & 100.2 \\
\hline M0116 & full & exp02 & 2048bit-ECFP4 & GBM & 0.000 & 0.718 & 0.626 & 0.011 & 0.011 & 100.1 \\
\hline M0117 & full & $\operatorname{exp03}$ & 2048bit-ECFP4 & GBM & 0.025 & 0.658 & 0.621 & 0.013 & 0.012 & 100.5 \\
\hline M0118 & full & exp04 & 2048bit-ECFP4 & GBM & 0.002 & 0.917 & 0.740 & 0.014 & 0.012 & 1000.9 \\
\hline M0119 & full & $\exp 05$ & 2048bit-ECFP4 & GBM & 0.000 & 1.056 & 0.802 & 0.023 & 0.023 & 7000.4 \\
\hline M0120 & full & exp06 & 2048bit-ECFP4 & GBM & 0.010 & 0.896 & 0.719 & 0.011 & 0.001 & 500.4 \\
\hline M0121 & full & exp07 & 2048bit-ECFP4 & GBM & 0.014 & 0.679 & 0.615 & 0.025 & 0.011 & 100.1 \\
\hline M0122 & full & exp08 & 2048bit-ECFP4 & GBM & 0.002 & 0.682 & 0.621 & 0.023 & 0.021 & 100.1 \\
\hline M0123 & full & exp09 & 2048bit-ECFP4 & GBM & 0.026 & 0.883 & 0.721 & 0.019 & 0.007 & 10000.9 \\
\hline M0124 & full & $\exp 10$ & 2048bit-ECFP4 & GBM & 0.007 & 0.977 & 0.741 & 0.037 & 0.030 & 500.8 \\
\hline M0125 & full & $\exp 11$ & 2048bit-ECFP4 & GBM & 0.038 & 0.964 & 0.734 & 0.009 & 0.029 & 500.6 \\
\hline M0126 & full & $\exp 12$ & 2048bit-ECFP4 & GBM & 0.020 & 1.025 & 0.784 & 0.015 & 0.005 & 6000.1 \\
\hline M0127 & full & exp13 & 2048bit-ECFP4 & GBM & 0.000 & 0.779 & 0.658 & 0.012 & 0.012 & 100.8 \\
\hline
\end{tabular}


Supporting Information (Texts S1-S9, Figures S1-S41 and Tables S1-S12)

\begin{tabular}{|c|c|c|c|c|c|c|c|c|c|c|}
\hline M0128 & full & $\exp 14$ & 2048bit-ECFP4 & GBM & 0.022 & 0.900 & 0.731 & 0.013 & 0.009 & 6000.7 \\
\hline M0129 & full & exp15 & 2048bit-ECFP4 & GBM & 0.000 & 0.925 & 0.744 & 0.012 & 0.012 & 1000.5 \\
\hline M0130 & full & exp16 & 2048bit-ECFP4 & GBM & 0.005 & 0.667 & 0.615 & 0.028 & 0.023 & 100.1 \\
\hline M0131 & full & exp17 & 2048bit-ECFP4 & GBM & 0.002 & 1.115 & 0.813 & 0.021 & 0.019 & 10000.9 \\
\hline M0132 & full & exp18 & 2048bit-ECFP4 & GBM & 0.023 & 0.668 & 0.627 & 0.013 & 0.010 & 100.5 \\
\hline M0133 & full & exp19 & 2048bit-ECFP4 & GBM & 0.013 & 0.856 & 0.689 & 0.017 & 0.004 & 100.5 \\
\hline M0134 & full & exp01 & 2048bit-ECFP4 & $\mathrm{RF}$ & 0.030 & 0.731 & 0.652 & 0.007 & 0.023 & 10 \\
\hline M0135 & full & exp02 & 2048bit-ECFP4 & $\mathrm{RF}$ & 0.001 & 0.817 & 0.692 & 0.011 & 0.010 & 10 \\
\hline M0136 & full & exp03 & 2048bit-ECFP4 & $\mathrm{RF}$ & 0.059 & 0.651 & 0.627 & 0.004 & 0.055 & 700 \\
\hline M0137 & full & exp04 & 2048bit-ECFP4 & $\mathrm{RF}$ & 0.007 & 0.739 & 0.663 & 0.009 & 0.002 & 200 \\
\hline M0138 & full & exp05 & 2048bit-ECFP4 & $\mathrm{RF}$ & 0.000 & 0.794 & 0.706 & 0.020 & 0.020 & 600 \\
\hline M0139 & full & exp06 & 2048bit-ECFP4 & $\mathrm{RF}$ & 0.002 & 0.897 & 0.737 & 0.010 & 0.008 & 300 \\
\hline M0140 & full & exp07 & 2048bit-ECFP4 & $\mathrm{RF}$ & 0.003 & 0.832 & 0.687 & 0.009 & 0.006 & 10 \\
\hline M0141 & full & exp08 & 2048bit-ECFP4 & $\mathrm{RF}$ & 0.003 & 0.840 & 0.719 & 0.008 & 0.005 & 10 \\
\hline M0142 & full & exp09 & 2048bit-ECFP4 & $\mathrm{RF}$ & 0.036 & 0.684 & 0.630 & 0.032 & 0.004 & 200 \\
\hline M0143 & full & exp10 & 2048bit-ECFP4 & $\mathrm{RF}$ & 0.005 & 0.858 & 0.707 & 0.021 & 0.016 & 700 \\
\hline M0144 & full & exp11 & 2048bit-ECFP4 & $\mathrm{RF}$ & 0.023 & 0.992 & 0.765 & 0.005 & 0.018 & 10 \\
\hline M0145 & full & exp12 & 2048bit-ECFP4 & $\mathrm{RF}$ & 0.006 & 0.842 & 0.714 & 0.013 & 0.007 & 10 \\
\hline M0146 & full & $\exp 13$ & 2048bit-ECFP4 & $\mathrm{RF}$ & 0.001 & 0.817 & 0.694 & 0.006 & 0.005 & 10 \\
\hline M0147 & full & exp14 & 2048bit-ECFP4 & $\mathrm{RF}$ & 0.046 & 0.694 & 0.623 & 0.010 & 0.036 & 400 \\
\hline M0148 & full & $\exp 15$ & 2048bit-ECFP4 & $\mathrm{RF}$ & 0.004 & 0.861 & 0.721 & 0.003 & 0.001 & 300 \\
\hline M0149 & full & exp16 & 2048bit-ECFP4 & $\mathrm{RF}$ & 0.005 & 0.733 & 0.647 & 0.028 & 0.023 & 700 \\
\hline M0150 & full & exp17 & 2048bit-ECFP4 & $\mathrm{RF}$ & 0.002 & 0.878 & 0.715 & 0.017 & 0.015 & 400 \\
\hline M0151 & full & exp18 & 2048bit-ECFP4 & $\mathrm{RF}$ & 0.017 & 0.730 & 0.669 & 0.003 & 0.014 & 10 \\
\hline M0152 & full & $\exp 19$ & 2048bit-ECFP4 & $\mathrm{RF}$ & 0.037 & 1.058 & 0.774 & 0.011 & 0.026 & 10 \\
\hline M0153 & full & exp01 & 1024bit-ECFP6 & KNN & 0.038 & 0.719 & 0.641 & 0.019 & 0.019 & 5 distance \\
\hline M0154 & full & exp02 & 1024bit-ECFP6 & KNN & 0.001 & 0.888 & 0.728 & 0.011 & 0.010 & 3 distance \\
\hline M0155 & full & exp03 & 1024bit-ECFP6 & KNN & 0.082 & 0.795 & 0.691 & 0.010 & 0.072 & 1 uniform \\
\hline M0156 & full & exp04 & 1024bit-ECFP6 & KNN & 0.001 & 1.185 & 0.832 & 0.018 & 0.017 & 1 uniform \\
\hline M0157 & full & $\exp 05$ & 1024bit-ECFP6 & KNN & 0.030 & 0.688 & 0.638 & 0.033 & 0.003 & 13 distance \\
\hline M0158 & full & exp06 & 1024bit-ECFP6 & KNN & 0.011 & 0.761 & 0.661 & 0.025 & 0.014 & 5 uniform \\
\hline M0159 & full & exp07 & 1024bit-ECFP6 & KNN & 0.004 & 0.693 & 0.628 & 0.012 & 0.008 & 15 uniform \\
\hline M0160 & full & exp08 & 1024bit-ECFP6 & KNN & 0.028 & 0.971 & 0.787 & 0.013 & 0.015 & 3 uniform \\
\hline M0161 & full & exp09 & 1024bit-ECFP6 & KNN & 0.038 & 0.690 & 0.646 & 0.028 & 0.010 & 3 uniform \\
\hline M0162 & full & $\exp 10$ & 1024bit-ECFP6 & KNN & 0.006 & 0.799 & 0.658 & 0.028 & 0.022 & 9 uniform \\
\hline M0163 & full & $\exp 11$ & 1024bit-ECFP6 & KNN & 0.031 & 0.805 & 0.677 & 0.015 & 0.016 & 9 uniform \\
\hline M0164 & full & $\exp 12$ & 1024bit-ECFP6 & KNN & 0.000 & 0.885 & 0.742 & 0.010 & 0.010 & 3 distance \\
\hline M0165 & full & exp13 & 1024bit-ECFP6 & KNN & 0.023 & 0.698 & 0.636 & 0.011 & 0.012 & 7 uniform \\
\hline M0166 & full & exp14 & 1024bit-ECFP6 & KNN & 0.059 & 0.678 & 0.618 & 0.028 & 0.031 & 3 uniform \\
\hline M0167 & full & exp15 & 1024bit-ECFP6 & KNN & 0.015 & 0.741 & 0.668 & 0.011 & 0.004 & 5 uniform \\
\hline M0168 & full & exp16 & 1024bit-ECFP6 & KNN & 0.017 & 0.991 & 0.754 & 0.028 & 0.011 & 1 uniform \\
\hline M0169 & full & $\exp 17$ & 1024bit-ECFP6 & KNN & 0.002 & 0.897 & 0.734 & 0.023 & 0.021 & 3 distance \\
\hline M0170 & full & $\exp 18$ & 1024bit-ECFP6 & KNN & 0.012 & 0.671 & 0.627 & 0.017 & 0.005 & 15 uniform \\
\hline M0171 & full & $\exp 19$ & 1024bit-ECFP6 & KNN & 0.054 & 1.120 & 0.788 & 0.014 & 0.040 & 3 uniform \\
\hline M0172 & full & exp01 & 1024bit-ECFP6 & SVM & 0.013 & 0.685 & 0.609 & 0.015 & 0.002 & 10.001 \\
\hline
\end{tabular}


Supporting Information (Texts S1-S9, Figures S1-S41 and Tables S1-S12)

\begin{tabular}{|c|c|c|c|c|c|c|c|c|c|c|}
\hline M0173 & full & exp02 & 1024bit-ECFP6 & SVM & 0.002 & 0.843 & 0.692 & 0.012 & 0.010 & 50.05 \\
\hline M0174 & full & exp03 & 1024bit-ECFP6 & SVM & 0.016 & 0.704 & 0.648 & 0.011 & 0.005 & 500.0005 \\
\hline M0175 & full & exp04 & 1024bit-ECFP6 & SVM & 0.001 & 0.766 & 0.676 & 0.014 & 0.013 & 50.05 \\
\hline M0176 & full & exp05 & 1024bit-ECFP6 & SVM & 0.010 & 0.890 & 0.744 & 0.026 & 0.016 & 500.01 \\
\hline M0177 & full & exp06 & 1024bit-ECFP6 & SVM & 0.000 & 0.783 & 0.667 & 0.022 & 0.022 & 10.05 \\
\hline M0178 & full & exp07 & 1024bit-ECFP6 & SVM & 0.001 & 1.434 & 0.923 & 0.015 & 0.014 & $50000.01 \mathrm{e}-05$ \\
\hline M0179 & full & exp08 & 1024bit-ECFP6 & SVM & 0.020 & 0.693 & 0.630 & 0.018 & 0.002 & $1001 \mathrm{e}-05$ \\
\hline M0180 & full & exp09 & 1024bit-ECFP6 & SVM & 0.017 & 0.744 & 0.675 & 0.028 & 0.011 & 100.05 \\
\hline M0181 & full & exp10 & 1024bit-ECFP6 & SVM & 0.015 & 0.808 & 0.665 & 0.023 & 0.008 & $1005 \mathrm{e}-05$ \\
\hline M0182 & full & exp11 & 1024bit-ECFP6 & SVM & 0.031 & 1.105 & 0.816 & 0.012 & 0.019 & $10000.01 \mathrm{e}-05$ \\
\hline M0183 & full & exp12 & 1024bit-ECFP6 & SVM & 0.002 & 0.726 & 0.645 & 0.019 & 0.017 & 10.01 \\
\hline M0184 & full & exp13 & 1024bit-ECFP6 & SVM & 0.005 & 1.441 & 0.947 & 0.012 & 0.007 & $100000.05 \mathrm{e}-05$ \\
\hline M0185 & full & $\exp 14$ & 1024bit-ECFP6 & SVM & 0.019 & 0.699 & 0.614 & 0.024 & 0.005 & 100.001 \\
\hline M0186 & full & $\exp 15$ & 1024bit-ECFP6 & SVM & 0.000 & 0.872 & 0.722 & 0.006 & 0.006 & 100.01 \\
\hline M0187 & full & $\exp 16$ & 1024bit-ECFP6 & SVM & 0.022 & 0.677 & 0.617 & 0.034 & 0.012 & 50.05 \\
\hline M0188 & full & exp17 & 1024bit-ECFP6 & SVM & 0.000 & 0.948 & 0.766 & 0.027 & 0.027 & 1000.01 \\
\hline M0189 & full & exp18 & 1024bit-ECFP6 & SVM & 0.001 & 1.109 & 0.831 & 0.011 & 0.010 & $5000.05 \mathrm{e}-05$ \\
\hline M0190 & full & $\exp 19$ & 1024bit-ECFP6 & SVM & 0.003 & 1.122 & 0.807 & 0.007 & 0.004 & $10000.01 \mathrm{e}-05$ \\
\hline M0191 & full & exp01 & 1024bit-ECFP6 & GBM & 0.040 & 0.689 & 0.617 & 0.012 & 0.028 & 500.3 \\
\hline M0192 & full & exp02 & 1024bit-ECFP6 & GBM & 0.003 & 0.852 & 0.685 & 0.009 & 0.006 & 2000.1 \\
\hline M0193 & full & exp03 & 1024bit-ECFP6 & GBM & 0.024 & 0.674 & 0.628 & 0.027 & 0.003 & 100.9 \\
\hline M0194 & full & exp04 & 1024bit-ECFP6 & GBM & 0.002 & 0.837 & 0.700 & 0.019 & 0.017 & 500.6 \\
\hline M0195 & full & exp05 & 1024bit-ECFP6 & GBM & 0.016 & 0.683 & 0.638 & 0.024 & 0.008 & 500.2 \\
\hline M0196 & full & exp06 & 1024bit-ECFP6 & GBM & 0.011 & 1.154 & 0.829 & 0.009 & 0.002 & 3000.6 \\
\hline M0197 & full & exp07 & 1024bit-ECFP6 & GBM & 0.004 & 0.711 & 0.637 & 0.015 & 0.011 & 100.5 \\
\hline M0198 & full & exp08 & 1024bit-ECFP6 & GBM & 0.000 & 0.771 & 0.672 & 0.019 & 0.019 & 500.2 \\
\hline M0199 & full & exp09 & 1024bit-ECFP6 & GBM & 0.011 & 0.916 & 0.747 & 0.026 & 0.015 & 9000.2 \\
\hline M0200 & full & exp10 & 1024bit-ECFP6 & GBM & 0.001 & 0.805 & 0.681 & 0.027 & 0.026 & 500.4 \\
\hline M0201 & full & exp11 & 1024bit-ECFP6 & GBM & 0.048 & 0.975 & 0.748 & 0.014 & 0.034 & 500.4 \\
\hline M0202 & full & $\exp 12$ & 1024bit-ECFP6 & GBM & 0.000 & 0.783 & 0.658 & 0.015 & 0.015 & 500.4 \\
\hline M0203 & full & exp13 & 1024bit-ECFP6 & GBM & 0.000 & 0.764 & 0.656 & 0.018 & 0.018 & 100.5 \\
\hline M0204 & full & exp14 & 1024bit-ECFP6 & GBM & 0.016 & 0.897 & 0.742 & 0.013 & 0.003 & 1000.7 \\
\hline M0205 & full & $\exp 15$ & 1024bit-ECFP6 & GBM & 0.000 & 0.865 & 0.704 & 0.023 & 0.023 & 100.9 \\
\hline M0206 & full & exp16 & 1024bit-ECFP6 & GBM & 0.009 & 0.881 & 0.719 & 0.024 & 0.015 & 4000.4 \\
\hline M0207 & full & exp17 & 1024bit-ECFP6 & GBM & 0.000 & 0.750 & 0.648 & 0.028 & 0.028 & 100.4 \\
\hline M0208 & full & $\exp 18$ & 1024bit-ECFP6 & GBM & 0.009 & 0.662 & 0.609 & 0.014 & 0.005 & 100.1 \\
\hline M0209 & full & $\exp 19$ & 1024bit-ECFP6 & GBM & 0.015 & 0.843 & 0.690 & 0.009 & 0.005 & 100.6 \\
\hline M0210 & full & exp01 & 1024bit-ECFP6 & $\mathrm{RF}$ & 0.038 & 0.692 & 0.626 & 0.008 & 0.030 & 1000 \\
\hline M0211 & full & exp02 & 1024bit-ECFP6 & $\mathrm{RF}$ & 0.000 & 0.807 & 0.672 & 0.007 & 0.007 & 100 \\
\hline M0212 & full & exp03 & 1024bit-ECFP6 & $\mathrm{RF}$ & 0.040 & 0.675 & 0.634 & 0.007 & 0.033 & 10 \\
\hline M0213 & full & exp04 & 1024bit-ECFP6 & $\mathrm{RF}$ & 0.016 & 0.707 & 0.645 & 0.013 & 0.003 & 10 \\
\hline M0214 & full & $\exp 05$ & 1024bit-ECFP6 & $\mathrm{RF}$ & 0.009 & 0.733 & 0.667 & 0.027 & 0.018 & 50 \\
\hline M0215 & full & exp06 & 1024bit-ECFP6 & $\mathrm{RF}$ & 0.000 & 0.831 & 0.695 & 0.007 & 0.007 & 300 \\
\hline M0216 & full & exp07 & 1024bit-ECFP6 & $\mathrm{RF}$ & 0.008 & 0.762 & 0.663 & 0.006 & 0.002 & 100 \\
\hline M0217 & full & exp08 & 1024bit-ECFP6 & $\mathrm{RF}$ & 0.001 & 0.805 & 0.701 & 0.010 & 0.009 & 300 \\
\hline
\end{tabular}


Supporting Information (Texts S1-S9, Figures S1-S41 and Tables S1-S12)

\begin{tabular}{|c|c|c|c|c|c|c|c|c|c|c|}
\hline M0218 & full & exp09 & 1024bit-ECFP6 & $\mathrm{RF}$ & 0.038 & 0.683 & 0.631 & 0.025 & 0.013 & 300 \\
\hline M0219 & full & exp10 & 1024bit-ECFP6 & $\mathrm{RF}$ & 0.001 & 0.812 & 0.684 & 0.014 & 0.013 & 700 \\
\hline M0220 & full & exp11 & 1024bit-ECFP6 & $\mathrm{RF}$ & 0.039 & 0.922 & 0.729 & 0.004 & 0.035 & 300 \\
\hline M0221 & full & exp12 & 1024bit-ECFP6 & $\mathrm{RF}$ & 0.000 & 0.759 & 0.664 & 0.007 & 0.007 & 100 \\
\hline M0222 & full & exp13 & 1024bit-ECFP6 & $\mathrm{RF}$ & 0.002 & 0.808 & 0.674 & 0.007 & 0.005 & 10 \\
\hline M0223 & full & exp14 & 1024bit-ECFP6 & $\mathrm{RF}$ & 0.037 & 0.693 & 0.619 & 0.013 & 0.024 & 50 \\
\hline M0224 & full & exp15 & 1024bit-ECFP6 & $\mathrm{RF}$ & 0.000 & 0.809 & 0.686 & 0.005 & 0.005 & 400 \\
\hline M0225 & full & exp16 & 1024bit-ECFP6 & $\mathrm{RF}$ & 0.012 & 0.698 & 0.633 & 0.015 & 0.003 & 800 \\
\hline M0226 & full & exp17 & 1024bit-ECFP6 & $\mathrm{RF}$ & 0.001 & 0.830 & 0.695 & 0.020 & 0.019 & 400 \\
\hline M0227 & full & exp18 & 1024bit-ECFP6 & $\mathrm{RF}$ & 0.003 & 0.769 & 0.699 & 0.005 & 0.002 & 10 \\
\hline M0228 & full & exp19 & 1024bit-ECFP6 & $\mathrm{RF}$ & 0.016 & 0.966 & 0.743 & 0.006 & 0.010 & 10 \\
\hline M0229 & full & exp01 & 2048bit-ECFP6 & KNN & 0.060 & 0.655 & 0.599 & 0.021 & 0.039 & 7 uniform \\
\hline M0230 & full & $\operatorname{exp02}$ & 2048bit-ECFP6 & KNN & 0.002 & 0.889 & 0.727 & 0.011 & 0.009 & 3 distance \\
\hline M0231 & full & exp03 & 2048bit-ECFP6 & KNN & 0.105 & 0.604 & 0.596 & 0.008 & 0.097 & 13 uniform \\
\hline M0232 & full & exp04 & 2048bit-ECFP6 & KNN & 0.002 & 1.240 & 0.854 & 0.021 & 0.019 & 1 uniform \\
\hline M0233 & full & exp05 & 2048bit-ECFP6 & KNN & 0.039 & 0.683 & 0.635 & 0.031 & 0.008 & 11 distance \\
\hline M0234 & full & exp06 & 2048bit-ECFP6 & KNN & 0.012 & 0.759 & 0.658 & 0.033 & 0.021 & 5 uniform \\
\hline M0235 & full & exp07 & 2048bit-ECFP6 & KNN & 0.001 & 0.800 & 0.677 & 0.012 & 0.011 & 3 uniform \\
\hline M0236 & full & $\exp 08$ & 2048bit-ECFP6 & KNN & 0.010 & 0.991 & 0.797 & 0.010 & 0.000 & 3 distance \\
\hline M0237 & full & exp09 & 2048bit-ECFP6 & KNN & 0.043 & 0.729 & 0.664 & 0.029 & 0.014 & 3 distance \\
\hline M0238 & full & $\exp 10$ & 2048bit-ECFP6 & KNN & 0.006 & 0.798 & 0.655 & 0.034 & 0.028 & 9 uniform \\
\hline M0239 & full & exp11 & 2048bit-ECFP6 & KNN & 0.032 & 0.804 & 0.665 & 0.020 & 0.012 & 9 uniform \\
\hline M0240 & full & exp12 & 2048bit-ECFP6 & KNN & 0.002 & 0.747 & 0.671 & 0.008 & 0.006 & 15 distance \\
\hline M0241 & full & exp13 & 2048bit-ECFP6 & KNN & 0.021 & 0.702 & 0.632 & 0.010 & 0.011 & 7 uniform \\
\hline M0242 & full & exp14 & 2048bit-ECFP6 & KNN & 0.060 & 0.674 & 0.613 & 0.034 & 0.026 & 3 uniform \\
\hline M0243 & full & exp15 & 2048bit-ECFP6 & KNN & 0.001 & 0.834 & 0.712 & 0.011 & 0.010 & 3 uniform \\
\hline M0244 & full & exp16 & 2048bit-ECFP6 & KNN & 0.018 & 0.728 & 0.636 & 0.028 & 0.010 & 3 uniform \\
\hline M0245 & full & exp17 & 2048bit-ECFP6 & KNN & 0.001 & 0.890 & 0.728 & 0.023 & 0.022 & 3 distance \\
\hline M0246 & full & exp18 & 2048bit-ECFP6 & KNN & 0.015 & 0.665 & 0.620 & 0.015 & 0.000 & 15 uniform \\
\hline M0247 & full & exp19 & 2048bit-ECFP6 & KNN & 0.024 & 0.892 & 0.697 & 0.014 & 0.010 & 5 uniform \\
\hline M0248 & full & exp01 & 2048bit-ECFP6 & SVM & 0.011 & 1.165 & 0.844 & 0.017 & 0.006 & $100000.01 \mathrm{e}-05$ \\
\hline M0249 & full & exp02 & 2048bit-ECFP6 & SVM & 0.004 & 0.850 & 0.694 & 0.012 & 0.008 & 50.05 \\
\hline M0250 & full & exp03 & 2048bit-ECFP6 & SVM & 0.016 & 0.703 & 0.644 & 0.011 & 0.005 & $5005 \mathrm{e}-05$ \\
\hline M0251 & full & exp04 & 2048bit-ECFP6 & SVM & 0.000 & 0.767 & 0.676 & 0.014 & 0.014 & 50.05 \\
\hline M0252 & full & exp05 & 2048bit-ECFP6 & SVM & 0.001 & 0.854 & 0.732 & 0.026 & 0.025 & 50.01 \\
\hline M0253 & full & exp06 & 2048bit-ECFP6 & SVM & 0.000 & 0.781 & 0.666 & 0.023 & 0.023 & 10.05 \\
\hline M0254 & full & exp07 & 2048bit-ECFP6 & SVM & 0.007 & 0.681 & 0.621 & 0.011 & 0.004 & $1001 \mathrm{e}-05$ \\
\hline M0255 & full & exp08 & 2048bit-ECFP6 & SVM & 0.020 & 0.716 & 0.645 & 0.018 & 0.002 & $505 \mathrm{e}-05$ \\
\hline M0256 & full & exp09 & 2048bit-ECFP6 & SVM & 0.005 & 0.923 & 0.731 & 0.030 & 0.025 & 1000.01 \\
\hline M0257 & full & exp10 & 2048bit-ECFP6 & SVM & 0.017 & 0.811 & 0.666 & 0.026 & 0.009 & $5001 \mathrm{e}-05$ \\
\hline M0258 & full & exp11 & 2048bit-ECFP6 & SVM & 0.027 & 1.128 & 0.817 & 0.017 & 0.010 & $10000.01 \mathrm{e}-05$ \\
\hline M0259 & full & exp12 & 2048bit-ECFP6 & SVM & 0.006 & 0.849 & 0.713 & 0.020 & 0.014 & 500.0005 \\
\hline M0260 & full & exp13 & 2048bit-ECFP6 & SVM & 0.007 & 1.107 & 0.828 & 0.014 & 0.007 & $5000.05 \mathrm{e}-05$ \\
\hline M0261 & full & exp14 & 2048bit-ECFP6 & SVM & 0.025 & 0.851 & 0.701 & 0.026 & 0.001 & 500.005 \\
\hline M0262 & full & exp15 & 2048bit-ECFP6 & SVM & 0.000 & 0.850 & 0.713 & 0.006 & 0.006 & 100.01 \\
\hline
\end{tabular}


Supporting Information (Texts S1-S9, Figures S1-S41 and Tables S1-S12)

\begin{tabular}{|c|c|c|c|c|c|c|c|c|c|c|}
\hline M0263 & full & exp16 & 2048bit-ECFP6 & SVM & 0.023 & 0.675 & 0.615 & 0.033 & 0.010 & 50.05 \\
\hline M0264 & full & exp17 & 2048bit-ECFP6 & SVM & 0.006 & 1.267 & 0.888 & 0.032 & 0.026 & 5000.00 .0005 \\
\hline M0265 & full & exp18 & 2048bit-ECFP6 & SVM & 0.122 & 0.665 & 0.610 & 0.007 & 0.114 & $11 \mathrm{e}-05$ \\
\hline M0266 & full & exp19 & 2048bit-ECFP6 & SVM & 0.005 & 1.489 & 0.949 & 0.007 & 0.002 & 100000.00 .0001 \\
\hline M0267 & full & exp01 & 2048bit-ECFP6 & GBM & 0.001 & 0.696 & 0.618 & 0.023 & 0.022 & 100.1 \\
\hline M0268 & full & $\operatorname{exp02}$ & 2048bit-ECFP6 & GBM & 0.000 & 0.846 & 0.695 & 0.010 & 0.010 & 500.5 \\
\hline M0269 & full & exp03 & 2048bit-ECFP6 & GBM & 0.014 & 0.768 & 0.656 & 0.011 & 0.003 & 500.7 \\
\hline M0270 & full & exp04 & 2048bit-ECFP6 & GBM & 0.003 & 0.896 & 0.746 & 0.020 & 0.017 & 1000.6 \\
\hline M0271 & full & exp05 & 2048bit-ECFP6 & GBM & 0.001 & 0.830 & 0.709 & 0.037 & 0.036 & 3000.1 \\
\hline M0272 & full & exp06 & 2048bit-ECFP6 & GBM & 0.013 & 1.310 & 0.846 & 0.011 & 0.002 & 1000.8 \\
\hline M0273 & full & exp07 & 2048bit-ECFP6 & GBM & 0.003 & 0.684 & 0.612 & 0.018 & 0.015 & 100.1 \\
\hline M0274 & full & $\exp 08$ & 2048bit-ECFP6 & GBM & 0.006 & 0.690 & 0.631 & 0.026 & 0.020 & 100.4 \\
\hline M0275 & full & exp09 & 2048bit-ECFP6 & GBM & 0.024 & 0.934 & 0.732 & 0.021 & 0.003 & 10000.3 \\
\hline M0276 & full & exp10 & 2048bit-ECFP6 & GBM & 0.000 & 0.903 & 0.729 & 0.034 & 0.034 & 500.6 \\
\hline M0277 & full & exp11 & 2048bit-ECFP6 & GBM & 0.025 & 0.735 & 0.636 & 0.012 & 0.013 & 100.2 \\
\hline M0278 & full & exp12 & 2048bit-ECFP6 & GBM & 0.001 & 0.664 & 0.610 & 0.021 & 0.020 & 100.1 \\
\hline M0279 & full & exp13 & 2048bit-ECFP6 & GBM & 0.003 & 0.703 & 0.622 & 0.010 & 0.007 & 100.1 \\
\hline M0280 & full & exp14 & 2048bit-ECFP6 & GBM & 0.029 & 0.720 & 0.645 & 0.022 & 0.007 & 500.4 \\
\hline M0281 & full & exp15 & 2048bit-ECFP6 & GBM & 0.000 & 0.939 & 0.733 & 0.010 & 0.010 & 6000.1 \\
\hline M0282 & full & exp16 & 2048bit-ECFP6 & GBM & 0.030 & 0.865 & 0.720 & 0.034 & 0.004 & 2000.7 \\
\hline M0283 & full & exp17 & 2048bit-ECFP6 & GBM & 0.000 & 0.763 & 0.645 & 0.028 & 0.028 & 100.6 \\
\hline M0284 & full & exp18 & 2048bit-ECFP6 & GBM & 0.001 & 0.668 & 0.610 & 0.023 & 0.022 & 100.1 \\
\hline M0285 & full & exp19 & 2048bit-ECFP6 & GBM & 0.009 & 1.157 & 0.802 & 0.009 & 0.000 & 1000.5 \\
\hline M0286 & full & exp01 & 2048bit-ECFP6 & $\mathrm{RF}$ & 0.022 & 0.720 & 0.640 & 0.011 & 0.011 & 300 \\
\hline M0287 & full & exp02 & 2048bit-ECFP6 & $\mathrm{RF}$ & 0.001 & 0.795 & 0.664 & 0.006 & 0.005 & 300 \\
\hline M0288 & full & exp03 & 2048bit-ECFP6 & $\mathrm{RF}$ & 0.030 & 0.689 & 0.636 & 0.003 & 0.027 & 10 \\
\hline M0289 & full & exp04 & 2048bit-ECFP6 & $\mathrm{RF}$ & 0.006 & 0.728 & 0.653 & 0.013 & 0.007 & 300 \\
\hline M0290 & full & exp05 & 2048bit-ECFP6 & $\mathrm{RF}$ & 0.004 & 0.789 & 0.698 & 0.035 & 0.031 & 10 \\
\hline M0291 & full & exp06 & 2048bit-ECFP6 & $\mathrm{RF}$ & 0.003 & 1.003 & 0.745 & 0.007 & 0.004 & 10 \\
\hline M0292 & full & exp07 & 2048bit-ECFP6 & $\mathrm{RF}$ & 0.004 & 0.779 & 0.670 & 0.006 & 0.002 & 900 \\
\hline M0293 & full & exp08 & 2048bit-ECFP6 & $\mathrm{RF}$ & 0.000 & 0.803 & 0.709 & 0.013 & 0.013 & 300 \\
\hline M0294 & full & exp09 & 2048bit-ECFP6 & $\mathrm{RF}$ & 0.029 & 0.699 & 0.637 & 0.028 & 0.001 & 300 \\
\hline M0295 & full & exp10 & 2048bit-ECFP6 & $\mathrm{RF}$ & 0.002 & 0.833 & 0.700 & 0.014 & 0.012 & 1000 \\
\hline M0296 & full & exp11 & 2048bit-ECFP6 & $\mathrm{RF}$ & 0.040 & 0.931 & 0.738 & 0.008 & 0.032 & 50 \\
\hline M0297 & full & exp12 & 2048bit-ECFP6 & $\mathrm{RF}$ & 0.005 & 0.804 & 0.688 & 0.007 & 0.002 & 300 \\
\hline M0298 & full & exp13 & 2048bit-ECFP6 & $\mathrm{RF}$ & 0.000 & 0.811 & 0.696 & 0.014 & 0.014 & 10 \\
\hline M0299 & full & exp14 & 2048bit-ECFP6 & $\mathrm{RF}$ & 0.034 & 0.703 & 0.629 & 0.015 & 0.019 & 400 \\
\hline M0300 & full & exp15 & 2048bit-ECFP6 & $\mathrm{RF}$ & 0.001 & 0.828 & 0.705 & 0.004 & 0.003 & 50 \\
\hline M0301 & full & exp16 & 2048bit-ECFP6 & $\mathrm{RF}$ & 0.022 & 0.679 & 0.631 & 0.031 & 0.009 & 800 \\
\hline M0302 & full & exp17 & 2048bit-ECFP6 & $\mathrm{RF}$ & 0.001 & 0.840 & 0.694 & 0.027 & 0.026 & 200 \\
\hline M0303 & full & exp18 & 2048bit-ECFP6 & $\mathrm{RF}$ & 0.004 & 0.766 & 0.694 & 0.004 & 0.000 & 10 \\
\hline M0304 & full & exp19 & 2048bit-ECFP6 & $\mathrm{RF}$ & 0.012 & 0.951 & 0.733 & 0.006 & 0.006 & 10 \\
\hline M0305 & 512 & exp01 & 1024bit-ECFP4 & KNN & 0.042 & 0.748 & 0.659 & 0.025 & 0.017 & 3 distance \\
\hline M0306 & 512 & exp02 & 1024bit-ECFP4 & KNN & 0.001 & 0.831 & 0.701 & 0.013 & 0.012 & 13 distance \\
\hline M0307 & 512 & $\exp 03$ & 1024bit-ECFP4 & KNN & 0.037 & 0.681 & 0.648 & 0.008 & 0.029 & 3 uniform \\
\hline
\end{tabular}


Supporting Information (Texts S1-S9, Figures S1-S41 and Tables S1-S12)

\begin{tabular}{|c|c|c|c|c|c|c|c|c|c|c|}
\hline M0308 & 512 & exp04 & 1024bit-ECFP4 & KNN & 0.000 & 1.112 & 0.790 & 0.013 & 0.013 & 1 uniform \\
\hline M0309 & 512 & exp05 & 1024bit-ECFP4 & KNN & 0.007 & 0.755 & 0.675 & 0.036 & 0.029 & 11 distance \\
\hline M0310 & 512 & exp06 & 1024bit-ECFP4 & KNN & 0.003 & 0.803 & 0.680 & 0.025 & 0.022 & 9 distance \\
\hline M0311 & 512 & exp07 & 1024bit-ECFP4 & KNN & 0.004 & 0.794 & 0.681 & 0.015 & 0.011 & 3 uniform \\
\hline M0312 & 512 & exp08 & 1024bit-ECFP4 & KNN & 0.008 & 0.875 & 0.732 & 0.013 & 0.005 & 9 distance \\
\hline M0313 & 512 & exp09 & 1024bit-ECFP4 & KNN & 0.027 & 0.750 & 0.662 & 0.028 & 0.001 & 3 distance \\
\hline M0314 & 512 & $\exp 10$ & 1024bit-ECFP4 & KNN & 0.003 & 0.784 & 0.663 & 0.026 & 0.023 & 9 uniform \\
\hline M0315 & 512 & $\exp 11$ & 1024bit-ECFP4 & KNN & 0.046 & 0.800 & 0.661 & 0.013 & 0.033 & 13 uniform \\
\hline M0316 & 512 & exp12 & 1024bit-ECFP4 & KNN & 0.002 & 1.155 & 0.821 & 0.010 & 0.008 & 1 distance \\
\hline M0317 & 512 & exp13 & 1024bit-ECFP4 & KNN & 0.015 & 0.731 & 0.640 & 0.008 & 0.007 & 5 uniform \\
\hline M0318 & 512 & exp14 & 1024bit-ECFP4 & KNN & 0.046 & 0.698 & 0.616 & 0.025 & 0.021 & 3 uniform \\
\hline M0319 & 512 & exp15 & 1024bit-ECFP4 & KNN & 0.003 & 0.994 & 0.783 & 0.007 & 0.004 & 1 uniform \\
\hline M0320 & 512 & $\exp 16$ & 1024bit-ECFP4 & KNN & 0.018 & 0.759 & 0.656 & 0.027 & 0.009 & 3 distance \\
\hline M0321 & 512 & $\exp 17$ & 1024bit-ECFP4 & KNN & 0.003 & 0.885 & 0.729 & 0.027 & 0.024 & 5 distance \\
\hline M0322 & 512 & $\exp 18$ & 1024bit-ECFP4 & KNN & 0.019 & 0.662 & 0.620 & 0.009 & 0.010 & 13 uniform \\
\hline M0323 & 512 & exp19 & 1024bit-ECFP4 & KNN & 0.003 & 1.530 & 0.921 & 0.013 & 0.010 & 1 uniform \\
\hline M0324 & 512 & exp01 & 1024bit-ECFP4 & SVM & 0.033 & 0.679 & 0.625 & 0.013 & 0.021 & 10.1 \\
\hline M0325 & 512 & $\exp 02$ & 1024bit-ECFP4 & SVM & 0.004 & 0.883 & 0.710 & 0.012 & 0.008 & 100.1 \\
\hline M0326 & 512 & $\exp 03$ & 1024bit-ECFP4 & SVM & 0.002 & 1.464 & 0.938 & 0.020 & 0.018 & 50000.00 .0005 \\
\hline M0327 & 512 & exp04 & 1024bit-ECFP4 & SVM & 0.001 & 0.774 & 0.674 & 0.012 & 0.011 & 50.1 \\
\hline M0328 & 512 & exp05 & 1024bit-ECFP4 & SVM & 0.002 & 0.857 & 0.732 & 0.023 & 0.021 & 50.05 \\
\hline M0329 & 512 & exp06 & 1024bit-ECFP4 & SVM & 0.000 & 0.791 & 0.677 & 0.021 & 0.021 & 10.1 \\
\hline M0330 & 512 & exp07 & 1024bit-ECFP4 & SVM & 0.000 & 0.716 & 0.639 & 0.018 & 0.018 & $1005 \mathrm{e}-05$ \\
\hline M0331 & 512 & exp08 & 1024bit-ECFP4 & SVM & 0.022 & 0.697 & 0.635 & 0.016 & 0.006 & $505 \mathrm{e}-05$ \\
\hline M0332 & 512 & exp09 & 1024bit-ECFP4 & SVM & 0.016 & 0.800 & 0.683 & 0.026 & 0.010 & 1000.05 \\
\hline M0333 & 512 & $\exp 10$ & 1024bit-ECFP4 & SVM & 0.014 & 1.309 & 0.877 & 0.075 & 0.061 & $50000.01 \mathrm{e}-05$ \\
\hline M0334 & 512 & $\exp 11$ & 1024bit-ECFP4 & SVM & 0.020 & 0.935 & 0.746 & 0.019 & 0.001 & 1000.001 \\
\hline M0335 & 512 & exp12 & 1024bit-ECFP4 & SVM & 0.000 & 0.842 & 0.712 & 0.021 & 0.021 & 1000.001 \\
\hline M0336 & 512 & exp13 & 1024bit-ECFP4 & SVM & 0.086 & 0.677 & 0.610 & 0.009 & 0.077 & $101 \mathrm{e}-05$ \\
\hline M0337 & 512 & $\exp 14$ & 1024bit-ECFP4 & SVM & 0.027 & 0.692 & 0.615 & 0.020 & 0.007 & 50.005 \\
\hline M0338 & 512 & $\exp 15$ & 1024bit-ECFP4 & SVM & 0.190 & 0.690 & 0.615 & 0.008 & 0.182 & $11 \mathrm{e}-05$ \\
\hline M0339 & 512 & $\exp 16$ & 1024bit-ECFP4 & SVM & 0.010 & 0.716 & 0.642 & 0.034 & 0.024 & 50.1 \\
\hline M0340 & 512 & $\exp 17$ & 1024bit-ECFP4 & SVM & 0.002 & 0.862 & 0.712 & 0.027 & 0.025 & 100000.00 .1 \\
\hline M0341 & 512 & exp18 & 1024bit-ECFP4 & SVM & 0.000 & 1.718 & 1.032 & 0.009 & 0.009 & $100000.05 \mathrm{e}-05$ \\
\hline M0342 & 512 & exp19 & 1024bit-ECFP4 & SVM & 0.014 & 1.782 & 1.030 & 0.018 & 0.004 & $50000.05 \mathrm{e}-05$ \\
\hline M0343 & 512 & exp01 & 1024bit-ECFP4 & GBM & 0.022 & 0.679 & 0.608 & 0.015 & 0.007 & 100.2 \\
\hline M0344 & 512 & $\exp 02$ & 1024bit-ECFP4 & GBM & 0.002 & 0.973 & 0.738 & 0.014 & 0.012 & 500.9 \\
\hline M0345 & 512 & exp03 & 1024bit-ECFP4 & GBM & 0.028 & 0.729 & 0.651 & 0.013 & 0.015 & 1000.6 \\
\hline M0346 & 512 & exp04 & 1024bit-ECFP4 & GBM & 0.001 & 0.944 & 0.731 & 0.017 & 0.016 & 9000.3 \\
\hline M0347 & 512 & exp05 & 1024bit-ECFP4 & GBM & 0.002 & 0.995 & 0.775 & 0.022 & 0.020 & 4000.8 \\
\hline M0348 & 512 & exp06 & 1024bit-ECFP4 & GBM & 0.027 & 1.161 & 0.828 & 0.019 & 0.008 & 10000.2 \\
\hline M0349 & 512 & exp07 & 1024bit-ECFP4 & GBM & 0.010 & 0.752 & 0.657 & 0.013 & 0.003 & 500.4 \\
\hline M0350 & 512 & $\exp 08$ & 1024bit-ECFP4 & GBM & 0.015 & 0.804 & 0.684 & 0.020 & 0.005 & 100.8 \\
\hline M0351 & 512 & exp09 & 1024bit-ECFP4 & GBM & 0.023 & 0.875 & 0.717 & 0.026 & 0.003 & 10000.6 \\
\hline M0352 & 512 & $\exp 10$ & 1024bit-ECFP4 & GBM & 0.002 & 0.834 & 0.689 & 0.052 & 0.050 & 500.3 \\
\hline
\end{tabular}


Supporting Information (Texts S1-S9, Figures S1-S41 and Tables S1-S12)

\begin{tabular}{|c|c|c|c|c|c|c|c|c|c|c|}
\hline M0353 & 512 & exp11 & 1024bit-ECFP4 & GBM & 0.024 & 0.915 & 0.717 & 0.010 & 0.014 & 500.4 \\
\hline M0354 & 512 & exp12 & 1024bit-ECFP4 & GBM & 0.022 & 0.958 & 0.734 & 0.015 & 0.007 & 500.6 \\
\hline M0355 & 512 & exp13 & 1024bit-ECFP4 & GBM & 0.001 & 0.701 & 0.627 & 0.014 & 0.013 & 100.2 \\
\hline M0356 & 512 & exp14 & 1024bit-ECFP4 & GBM & 0.020 & 0.896 & 0.745 & 0.013 & 0.007 & 10000.6 \\
\hline M0357 & 512 & exp15 & 1024bit-ECFP4 & GBM & 0.006 & 0.716 & 0.633 & 0.014 & 0.008 & 100.1 \\
\hline M0358 & 512 & exp16 & 1024bit-ECFP4 & GBM & 0.002 & 0.670 & 0.614 & 0.019 & 0.017 & 100.2 \\
\hline M0359 & 512 & exp17 & 1024bit-ECFP4 & GBM & 0.003 & 1.031 & 0.778 & 0.023 & 0.020 & 4000.2 \\
\hline M0360 & 512 & exp18 & 1024bit-ECFP4 & GBM & 0.001 & 0.806 & 0.700 & 0.011 & 0.010 & 500.5 \\
\hline M0361 & 512 & exp19 & 1024bit-ECFP4 & GBM & 0.019 & 1.292 & 0.838 & 0.015 & 0.004 & 500.8 \\
\hline M0362 & 512 & exp01 & 1024bit-ECFP4 & $\mathrm{RF}$ & 0.046 & 0.697 & 0.623 & 0.009 & 0.037 & 600 \\
\hline M0363 & 512 & exp02 & 1024bit-ECFP4 & $\mathrm{RF}$ & 0.001 & 0.819 & 0.692 & 0.011 & 0.010 & 10 \\
\hline M0364 & 512 & $\exp 03$ & 1024bit-ECFP4 & $\mathrm{RF}$ & 0.041 & 0.682 & 0.649 & 0.006 & 0.035 & 10 \\
\hline M0365 & 512 & exp04 & 1024bit-ECFP4 & $\mathrm{RF}$ & 0.006 & 0.742 & 0.665 & 0.013 & 0.007 & 200 \\
\hline M0366 & 512 & exp05 & 1024bit-ECFP4 & $\mathrm{RF}$ & 0.007 & 0.753 & 0.686 & 0.020 & 0.013 & 300 \\
\hline M0367 & 512 & exp06 & 1024bit-ECFP4 & $\mathrm{RF}$ & 0.000 & 0.888 & 0.728 & 0.009 & 0.009 & 10 \\
\hline M0368 & 512 & exp07 & 1024bit-ECFP4 & $\mathrm{RF}$ & 0.013 & 0.781 & 0.672 & 0.008 & 0.005 & 10 \\
\hline M0369 & 512 & exp08 & 1024bit-ECFP4 & $\mathrm{RF}$ & 0.004 & 0.847 & 0.711 & 0.005 & 0.001 & 10 \\
\hline M0370 & 512 & exp09 & 1024bit-ECFP4 & $\mathrm{RF}$ & 0.034 & 0.687 & 0.625 & 0.033 & 0.001 & 100 \\
\hline M0371 & 512 & exp10 & 1024bit-ECFP4 & $\mathrm{RF}$ & 0.006 & 0.875 & 0.718 & 0.029 & 0.023 & 700 \\
\hline M0372 & 512 & exp11 & 1024bit-ECFP4 & $\mathrm{RF}$ & 0.032 & 1.002 & 0.761 & 0.003 & 0.029 & 10 \\
\hline M0373 & 512 & exp12 & 1024bit-ECFP4 & $\mathrm{RF}$ & 0.009 & 0.834 & 0.713 & 0.011 & 0.003 & 100 \\
\hline M0374 & 512 & exp13 & 1024bit-ECFP4 & $\mathrm{RF}$ & 0.003 & 0.815 & 0.695 & 0.006 & 0.003 & 10 \\
\hline M0375 & 512 & exp14 & 1024bit-ECFP4 & $\mathrm{RF}$ & 0.041 & 0.697 & 0.625 & 0.011 & 0.030 & 10 \\
\hline M0376 & 512 & $\operatorname{exp15}$ & 1024bit-ECFP4 & $\mathrm{RF}$ & 0.005 & 0.909 & 0.732 & 0.008 & 0.003 & 10 \\
\hline M0377 & 512 & exp16 & 1024bit-ECFP4 & $\mathrm{RF}$ & 0.002 & 0.745 & 0.655 & 0.026 & 0.024 & 100 \\
\hline M0378 & 512 & exp17 & 1024bit-ECFP4 & $\mathrm{RF}$ & 0.000 & 0.868 & 0.714 & 0.015 & 0.015 & 100 \\
\hline M0379 & 512 & exp18 & 1024bit-ECFP4 & $\mathrm{RF}$ & 0.004 & 0.764 & 0.684 & 0.003 & 0.001 & 10 \\
\hline M0380 & 512 & exp19 & 1024bit-ECFP4 & $\mathrm{RF}$ & 0.028 & 1.041 & 0.761 & 0.011 & 0.017 & 10 \\
\hline M0381 & 512 & exp01 & 2048bit-ECFP4 & KNN & 0.016 & 0.747 & 0.646 & 0.021 & 0.005 & 7 distance \\
\hline M0382 & 512 & exp02 & 2048bit-ECFP4 & KNN & 0.001 & 0.833 & 0.697 & 0.013 & 0.012 & 9 distance \\
\hline M0383 & 512 & exp03 & 2048bit-ECFP4 & KNN & 0.058 & 0.653 & 0.633 & 0.010 & 0.048 & 3 uniform \\
\hline M0384 & 512 & exp04 & 2048bit-ECFP4 & KNN & 0.005 & 0.821 & 0.698 & 0.011 & 0.006 & 3 distance \\
\hline M0385 & 512 & exp05 & 2048bit-ECFP4 & KNN & 0.000 & 0.801 & 0.700 & 0.032 & 0.032 & 9 distance \\
\hline M0386 & 512 & exp06 & 2048bit-ECFP4 & KNN & 0.000 & 0.844 & 0.698 & 0.029 & 0.029 & 7 distance \\
\hline M0387 & 512 & exp07 & 2048bit-ECFP4 & KNN & 0.002 & 0.744 & 0.656 & 0.010 & 0.008 & 5 uniform \\
\hline M0388 & 512 & $\exp 08$ & 2048bit-ECFP4 & KNN & 0.001 & 0.857 & 0.725 & 0.011 & 0.010 & 9 distance \\
\hline M0389 & 512 & exp09 & 2048bit-ECFP4 & KNN & 0.031 & 0.751 & 0.670 & 0.029 & 0.002 & 3 distance \\
\hline M0390 & 512 & exp10 & 2048bit-ECFP4 & KNN & 0.000 & 0.769 & 0.657 & 0.022 & 0.022 & 9 uniform \\
\hline M0391 & 512 & exp11 & 2048bit-ECFP4 & KNN & 0.039 & 0.783 & 0.658 & 0.011 & 0.028 & 15 uniform \\
\hline M0392 & 512 & exp12 & 2048bit-ECFP4 & KNN & 0.008 & 0.801 & 0.692 & 0.009 & 0.001 & 15 distance \\
\hline M0393 & 512 & exp13 & 2048bit-ECFP4 & KNN & 0.013 & 0.687 & 0.605 & 0.012 & 0.001 & 15 uniform \\
\hline M0394 & 512 & exp14 & 2048bit-ECFP4 & KNN & 0.036 & 0.684 & 0.610 & 0.026 & 0.010 & 5 uniform \\
\hline M0395 & 512 & exp15 & 2048bit-ECFP4 & KNN & 0.000 & 0.728 & 0.664 & 0.007 & 0.007 & 13 uniform \\
\hline M0396 & 512 & exp16 & 2048bit-ECFP4 & KNN & 0.003 & 1.068 & 0.782 & 0.030 & 0.027 & 1 uniform \\
\hline M0397 & 512 & exp17 & 2048bit-ECFP4 & KNN & 0.002 & 0.924 & 0.739 & 0.027 & 0.025 & 3 distance \\
\hline
\end{tabular}


Supporting Information (Texts S1-S9, Figures S1-S41 and Tables S1-S12)

\begin{tabular}{|c|c|c|c|c|c|c|c|c|c|c|}
\hline M0398 & 512 & $\exp 18$ & 2048bit-ECFP4 & KNN & 0.016 & 0.663 & 0.616 & 0.011 & 0.005 & 13 uniform \\
\hline M0399 & 512 & exp19 & 2048bit-ECFP4 & KNN & 0.024 & 0.807 & 0.677 & 0.009 & 0.015 & 9 uniform \\
\hline M0400 & 512 & exp01 & 2048bit-ECFP4 & SVM & 0.004 & 0.707 & 0.632 & 0.013 & 0.009 & 10.01 \\
\hline M0401 & 512 & exp02 & 2048bit-ECFP4 & SVM & 0.003 & 0.885 & 0.713 & 0.010 & 0.007 & 100.1 \\
\hline M0402 & 512 & exp03 & 2048bit-ECFP4 & SVM & 0.011 & 0.707 & 0.648 & 0.020 & 0.009 & $5000.01 \mathrm{e}-05$ \\
\hline M0403 & 512 & exp04 & 2048bit-ECFP4 & SVM & 0.011 & 1.615 & 0.999 & 0.013 & 0.002 & 100000.00 .0001 \\
\hline M0404 & 512 & exp05 & 2048bit-ECFP4 & SVM & 0.001 & 0.871 & 0.740 & 0.020 & 0.019 & 50.05 \\
\hline M0405 & 512 & exp06 & 2048bit-ECFP4 & SVM & 0.002 & 1.360 & 0.907 & 0.029 & 0.027 & 10000.00 .0001 \\
\hline M0406 & 512 & exp07 & 2048bit-ECFP4 & SVM & 0.001 & 0.747 & 0.663 & 0.018 & 0.017 & $5005 \mathrm{e}-05$ \\
\hline M0407 & 512 & exp08 & 2048bit-ECFP4 & SVM & 0.003 & 1.452 & 0.965 & 0.019 & 0.016 & $100000.05 \mathrm{e}-05$ \\
\hline M0408 & 512 & exp09 & 2048bit-ECFP4 & SVM & 0.014 & 0.812 & 0.685 & 0.027 & 0.013 & 1000.05 \\
\hline M0409 & 512 & $\exp 10$ & 2048bit-ECFP4 & SVM & 0.018 & 0.807 & 0.654 & 0.037 & 0.019 & $1000.01 \mathrm{e}-05$ \\
\hline M0410 & 512 & $\exp 11$ & 2048bit-ECFP4 & SVM & 0.016 & 0.955 & 0.757 & 0.018 & 0.002 & 1000.001 \\
\hline M0411 & 512 & $\exp 12$ & 2048bit-ECFP4 & SVM & 0.000 & 0.970 & 0.778 & 0.027 & 0.027 & 5000.0005 \\
\hline M0412 & 512 & exp13 & 2048bit-ECFP4 & SVM & 0.079 & 0.678 & 0.611 & 0.009 & 0.070 & $101 \mathrm{e}-05$ \\
\hline M0413 & 512 & exp14 & 2048bit-ECFP4 & SVM & 0.041 & 0.674 & 0.605 & 0.022 & 0.019 & 50.005 \\
\hline M0414 & 512 & exp15 & 2048bit-ECFP4 & SVM & 0.115 & 0.693 & 0.617 & 0.009 & 0.107 & $51 \mathrm{e}-05$ \\
\hline M0415 & 512 & $\exp 16$ & 2048bit-ECFP4 & SVM & 0.009 & 0.735 & 0.647 & 0.033 & 0.024 & 50.1 \\
\hline M0416 & 512 & $\exp 17$ & 2048bit-ECFP4 & SVM & 0.001 & 0.875 & 0.715 & 0.034 & 0.033 & 1000.00 .1 \\
\hline M0417 & 512 & $\exp 18$ & 2048bit-ECFP4 & SVM & 0.162 & 0.666 & 0.611 & 0.008 & 0.154 & $11 \mathrm{e}-05$ \\
\hline M0418 & 512 & exp19 & 2048bit-ECFP4 & SVM & 0.002 & 1.580 & 0.952 & 0.013 & 0.011 & $100000.05 \mathrm{e}-05$ \\
\hline M0419 & 512 & exp01 & 2048bit-ECFP4 & GBM & 0.025 & 0.815 & 0.707 & 0.021 & 0.004 & 1000.7 \\
\hline M0420 & 512 & exp02 & 2048bit-ECFP4 & GBM & 0.005 & 0.917 & 0.718 & 0.013 & 0.008 & 1000.3 \\
\hline M0421 & 512 & exp03 & 2048bit-ECFP4 & GBM & 0.051 & 0.648 & 0.601 & 0.012 & 0.039 & 100.1 \\
\hline M0422 & 512 & exp04 & 2048bit-ECFP4 & GBM & 0.010 & 0.845 & 0.717 & 0.011 & 0.001 & 1000.7 \\
\hline M0423 & 512 & exp05 & 2048bit-ECFP4 & GBM & 0.001 & 1.054 & 0.803 & 0.017 & 0.016 & 5000.3 \\
\hline M0424 & 512 & exp06 & 2048bit-ECFP4 & GBM & 0.012 & 1.095 & 0.819 & 0.016 & 0.004 & 3000.4 \\
\hline M0425 & 512 & exp07 & 2048bit-ECFP4 & GBM & 0.014 & 0.679 & 0.615 & 0.024 & 0.010 & 100.1 \\
\hline M0426 & 512 & exp08 & 2048bit-ECFP4 & GBM & 0.003 & 0.685 & 0.623 & 0.022 & 0.019 & 100.1 \\
\hline M0427 & 512 & exp09 & 2048bit-ECFP4 & GBM & 0.011 & 0.902 & 0.726 & 0.021 & 0.010 & 10000.6 \\
\hline M0428 & 512 & $\exp 10$ & 2048bit-ECFP4 & GBM & 0.007 & 0.829 & 0.674 & 0.041 & 0.034 & 500.3 \\
\hline M0429 & 512 & exp11 & 2048bit-ECFP4 & GBM & 0.029 & 0.974 & 0.752 & 0.011 & 0.018 & 500.6 \\
\hline M0430 & 512 & $\exp 12$ & 2048bit-ECFP4 & GBM & 0.004 & 0.830 & 0.696 & 0.014 & 0.010 & 1000.2 \\
\hline M0431 & 512 & exp13 & 2048bit-ECFP4 & GBM & 0.002 & 0.687 & 0.617 & 0.010 & 0.008 & 100.1 \\
\hline M0432 & 512 & exp14 & 2048bit-ECFP4 & GBM & 0.021 & 0.889 & 0.728 & 0.017 & 0.004 & 10000.6 \\
\hline M0433 & 512 & $\exp 15$ & 2048bit-ECFP4 & GBM & 0.003 & 0.905 & 0.736 & 0.021 & 0.018 & 500.5 \\
\hline M0434 & 512 & $\exp 16$ & 2048bit-ECFP4 & GBM & 0.004 & 0.665 & 0.613 & 0.030 & 0.026 & 100.1 \\
\hline M0435 & 512 & $\exp 17$ & 2048bit-ECFP4 & GBM & 0.003 & 1.096 & 0.801 & 0.029 & 0.026 & 10000.4 \\
\hline M0436 & 512 & $\exp 18$ & 2048bit-ECFP4 & GBM & 0.041 & 0.643 & 0.610 & 0.014 & 0.027 & 100.4 \\
\hline M0437 & 512 & exp19 & 2048bit-ECFP4 & GBM & 0.004 & 0.835 & 0.676 & 0.014 & 0.010 & 100.5 \\
\hline M0438 & 512 & exp01 & 2048bit-ECFP4 & $\mathrm{RF}$ & 0.025 & 0.737 & 0.650 & 0.011 & 0.015 & 10 \\
\hline M0439 & 512 & $\exp 02$ & 2048bit-ECFP4 & $\mathrm{RF}$ & 0.000 & 0.822 & 0.679 & 0.007 & 0.007 & 300 \\
\hline M0440 & 512 & $\exp 03$ & 2048bit-ECFP4 & $\mathrm{RF}$ & 0.062 & 0.649 & 0.628 & 0.003 & 0.059 & 700 \\
\hline M0441 & 512 & exp04 & 2048bit-ECFP4 & $\mathrm{RF}$ & 0.008 & 0.735 & 0.661 & 0.007 & 0.001 & 200 \\
\hline M0442 & 512 & exp05 & 2048bit-ECFP4 & $\mathrm{RF}$ & 0.000 & 0.799 & 0.708 & 0.020 & 0.020 & 100 \\
\hline
\end{tabular}


Supporting Information (Texts S1-S9, Figures S1-S41 and Tables S1-S12)

\begin{tabular}{|c|c|c|c|c|c|c|c|c|c|c|}
\hline M0443 & 512 & exp06 & 2048bit-ECFP4 & $\mathrm{RF}$ & 0.000 & 0.914 & 0.743 & 0.017 & 0.017 & 10 \\
\hline M0444 & 512 & exp07 & 2048bit-ECFP4 & $\mathrm{RF}$ & 0.002 & 0.854 & 0.697 & 0.009 & 0.007 & 10 \\
\hline M0445 & 512 & exp08 & 2048bit-ECFP4 & $\mathrm{RF}$ & 0.000 & 0.820 & 0.709 & 0.005 & 0.005 & 10 \\
\hline M0446 & 512 & exp09 & 2048bit-ECFP4 & $\mathrm{RF}$ & 0.036 & 0.686 & 0.630 & 0.029 & 0.007 & 50 \\
\hline M0447 & 512 & exp10 & 2048bit-ECFP4 & $\mathrm{RF}$ & 0.005 & 0.861 & 0.707 & 0.026 & 0.021 & 700 \\
\hline M0448 & 512 & $\exp 11$ & 2048bit-ECFP4 & $\mathrm{RF}$ & 0.024 & 0.997 & 0.771 & 0.006 & 0.018 & 10 \\
\hline M0449 & 512 & $\exp 12$ & 2048bit-ECFP4 & $\mathrm{RF}$ & 0.006 & 0.837 & 0.712 & 0.015 & 0.009 & 10 \\
\hline M0450 & 512 & exp13 & 2048bit-ECFP4 & $\mathrm{RF}$ & 0.000 & 0.813 & 0.685 & 0.004 & 0.004 & 400 \\
\hline M0451 & 512 & exp14 & 2048bit-ECFP4 & $\mathrm{RF}$ & 0.042 & 0.701 & 0.626 & 0.009 & 0.033 & 400 \\
\hline M0452 & 512 & exp15 & 2048bit-ECFP4 & $\mathrm{RF}$ & 0.002 & 0.856 & 0.720 & 0.004 & 0.002 & 700 \\
\hline M0453 & 512 & exp16 & 2048bit-ECFP4 & $\mathrm{RF}$ & 0.002 & 0.751 & 0.664 & 0.025 & 0.023 & 50 \\
\hline M0454 & 512 & exp17 & 2048bit-ECFP4 & $\mathrm{RF}$ & 0.002 & 0.878 & 0.714 & 0.021 & 0.019 & 200 \\
\hline M0455 & 512 & $\exp 18$ & 2048bit-ECFP4 & $\mathrm{RF}$ & 0.015 & 0.732 & 0.669 & 0.003 & 0.012 & 10 \\
\hline M0456 & 512 & exp19 & 2048bit-ECFP4 & $\mathrm{RF}$ & 0.041 & 1.071 & 0.776 & 0.009 & 0.032 & 10 \\
\hline M0457 & 512 & exp01 & 1024bit-ECFP6 & KNN & 0.010 & 0.754 & 0.651 & 0.020 & 0.010 & 9 distance \\
\hline M0458 & 512 & exp02 & 1024bit-ECFP6 & KNN & 0.004 & 0.851 & 0.702 & 0.016 & 0.012 & 3 distance \\
\hline M0459 & 512 & exp03 & 1024bit-ECFP6 & KNN & 0.090 & 0.741 & 0.666 & 0.008 & 0.082 & 1 distance \\
\hline M0460 & 512 & exp04 & 1024bit-ECFP6 & KNN & 0.005 & 1.034 & 0.787 & 0.017 & 0.012 & 1 uniform \\
\hline M0461 & 512 & $\exp 05$ & 1024bit-ECFP6 & KNN & 0.009 & 0.748 & 0.671 & 0.027 & 0.018 & 7 distance \\
\hline M0462 & 512 & exp06 & 1024bit-ECFP6 & KNN & 0.005 & 0.781 & 0.662 & 0.026 & 0.021 & 5 uniform \\
\hline M0463 & 512 & exp07 & 1024bit-ECFP6 & KNN & 0.001 & 1.049 & 0.790 & 0.013 & 0.012 & 1 distance \\
\hline M0464 & 512 & exp08 & 1024bit-ECFP6 & KNN & 0.033 & 0.949 & 0.771 & 0.011 & 0.022 & 3 uniform \\
\hline M0465 & 512 & exp09 & 1024bit-ECFP6 & KNN & 0.035 & 0.695 & 0.648 & 0.021 & 0.014 & 9 distance \\
\hline M0466 & 512 & $\exp 10$ & 1024bit-ECFP6 & KNN & 0.004 & 0.787 & 0.654 & 0.030 & 0.026 & 9 uniform \\
\hline M0467 & 512 & $\exp 11$ & 1024bit-ECFP6 & KNN & 0.021 & 0.794 & 0.665 & 0.010 & 0.011 & 11 uniform \\
\hline M0468 & 512 & $\exp 12$ & 1024bit-ECFP6 & KNN & 0.001 & 0.785 & 0.694 & 0.011 & 0.010 & 9 distance \\
\hline M0469 & 512 & exp13 & 1024bit-ECFP6 & KNN & 0.013 & 0.705 & 0.626 & 0.012 & 0.001 & 9 uniform \\
\hline M0470 & 512 & exp14 & 1024bit-ECFP6 & KNN & 0.049 & 0.669 & 0.606 & 0.027 & 0.022 & 5 uniform \\
\hline M0471 & 512 & exp15 & 1024bit-ECFP6 & KNN & 0.001 & 0.815 & 0.699 & 0.010 & 0.009 & 3 uniform \\
\hline M0472 & 512 & $\exp 16$ & 1024bit-ECFP6 & KNN & 0.024 & 0.698 & 0.627 & 0.028 & 0.004 & 3 uniform \\
\hline M0473 & 512 & $\exp 17$ & 1024bit-ECFP6 & KNN & 0.000 & 0.908 & 0.716 & 0.036 & 0.036 & 3 distance \\
\hline M0474 & 512 & $\exp 18$ & 1024bit-ECFP6 & KNN & 0.004 & 0.686 & 0.631 & 0.013 & 0.009 & 15 uniform \\
\hline M0475 & 512 & exp19 & 1024bit-ECFP6 & KNN & 0.043 & 0.808 & 0.672 & 0.014 & 0.029 & 11 uniform \\
\hline M0476 & 512 & exp01 & 1024bit-ECFP6 & SVM & 0.002 & 1.646 & 1.018 & 0.023 & 0.021 & 100000.00 .0001 \\
\hline M0477 & 512 & $\exp 02$ & 1024bit-ECFP6 & SVM & 0.019 & 1.197 & 0.829 & 0.015 & 0.004 & $5000.05 \mathrm{e}-05$ \\
\hline M0478 & 512 & exp03 & 1024bit-ECFP6 & SVM & 0.000 & 1.370 & 0.887 & 0.018 & 0.018 & $100000.01 \mathrm{e}-05$ \\
\hline M0479 & 512 & exp04 & 1024bit-ECFP6 & SVM & 0.003 & 1.974 & 1.050 & 0.015 & 0.011 & $50000.05 \mathrm{e}-05$ \\
\hline M0480 & 512 & exp05 & 1024bit-ECFP6 & SVM & 0.012 & 0.709 & 0.661 & 0.023 & 0.011 & 50.1 \\
\hline M0481 & 512 & exp06 & 1024bit-ECFP6 & SVM & 0.003 & 1.204 & 0.826 & 0.024 & 0.021 & 5000.001 \\
\hline M0482 & 512 & exp07 & 1024bit-ECFP6 & SVM & 0.001 & 1.262 & 0.872 & 0.019 & 0.018 & 5000.001 \\
\hline M0483 & 512 & exp08 & 1024bit-ECFP6 & SVM & 0.016 & 0.687 & 0.626 & 0.010 & 0.006 & $1001 \mathrm{e}-05$ \\
\hline M0484 & 512 & exp09 & 1024bit-ECFP6 & SVM & 0.012 & 0.775 & 0.685 & 0.025 & 0.013 & 100.05 \\
\hline M0485 & 512 & $\exp 10$ & 1024bit-ECFP6 & SVM & 0.007 & 0.824 & 0.682 & 0.030 & 0.023 & $1000.01 \mathrm{e}-05$ \\
\hline M0486 & 512 & $\exp 11$ & 1024bit-ECFP6 & SVM & 0.026 & 0.860 & 0.708 & 0.018 & 0.008 & $5005 \mathrm{e}-05$ \\
\hline M0487 & 512 & $\exp 12$ & 1024bit-ECFP6 & SVM & 0.000 & 0.768 & 0.674 & 0.024 & 0.024 & 500.0005 \\
\hline
\end{tabular}


Supporting Information (Texts S1-S9, Figures S1-S41 and Tables S1-S12)

\begin{tabular}{|c|c|c|c|c|c|c|c|c|c|c|}
\hline M0488 & 512 & $\exp 13$ & 1024bit-ECFP6 & SVM & 0.069 & 0.681 & 0.612 & 0.010 & 0.059 & $15 \mathrm{e}-05$ \\
\hline M0489 & 512 & exp14 & 1024bit-ECFP6 & SVM & 0.021 & 0.714 & 0.620 & 0.018 & 0.003 & 50.005 \\
\hline M0490 & 512 & exp15 & 1024bit-ECFP6 & SVM & 0.004 & 2.019 & 1.058 & 0.018 & 0.014 & $100000.05 \mathrm{e}-05$ \\
\hline M0491 & 512 & exp16 & 1024bit-ECFP6 & SVM & 0.019 & 0.707 & 0.628 & 0.029 & 0.010 & 100.05 \\
\hline M0492 & 512 & exp17 & 1024bit-ECFP6 & SVM & 0.001 & 1.011 & 0.787 & 0.032 & 0.031 & 1000.00 .01 \\
\hline M0493 & 512 & $\exp 18$ & 1024bit-ECFP6 & SVM & 0.000 & 1.167 & 0.869 & 0.010 & 0.010 & 1000.00 .001 \\
\hline M0494 & 512 & exp19 & 1024bit-ECFP6 & SVM & 0.044 & 0.778 & 0.662 & 0.011 & 0.033 & $505 \mathrm{e}-05$ \\
\hline M0495 & 512 & exp01 & 1024bit-ECFP6 & GBM & 0.036 & 0.766 & 0.686 & 0.014 & 0.022 & 500.7 \\
\hline M0496 & 512 & exp02 & 1024bit-ECFP6 & GBM & 0.000 & 0.751 & 0.641 & 0.023 & 0.023 & 100.4 \\
\hline M0497 & 512 & exp03 & 1024bit-ECFP6 & GBM & 0.038 & 0.643 & 0.610 & 0.021 & 0.017 & 100.4 \\
\hline M0498 & 512 & exp04 & 1024bit-ECFP6 & GBM & 0.014 & 0.722 & 0.656 & 0.010 & 0.004 & 500.3 \\
\hline M0499 & 512 & exp05 & 1024bit-ECFP6 & GBM & 0.010 & 0.732 & 0.662 & 0.018 & 0.008 & 500.3 \\
\hline M0500 & 512 & exp06 & 1024bit-ECFP6 & GBM & 0.007 & 1.106 & 0.805 & 0.013 & 0.006 & 2000.8 \\
\hline M0501 & 512 & exp07 & 1024bit-ECFP6 & GBM & 0.005 & 0.705 & 0.634 & 0.022 & 0.017 & 100.5 \\
\hline M0502 & 512 & exp08 & 1024bit-ECFP6 & GBM & 0.002 & 0.795 & 0.687 & 0.014 & 0.012 & 500.2 \\
\hline M0503 & 512 & exp09 & 1024bit-ECFP6 & GBM & 0.026 & 0.878 & 0.731 & 0.026 & 0.000 & 1000.8 \\
\hline M0504 & 512 & exp10 & 1024bit-ECFP6 & GBM & 0.000 & 0.844 & 0.699 & 0.035 & 0.035 & 500.4 \\
\hline M0505 & 512 & $\exp 11$ & 1024bit-ECFP6 & GBM & 0.032 & 0.803 & 0.664 & 0.020 & 0.012 & 100.4 \\
\hline M0506 & 512 & $\exp 12$ & 1024bit-ECFP6 & GBM & 0.000 & 0.928 & 0.743 & 0.012 & 0.012 & 2000.4 \\
\hline M0507 & 512 & exp13 & 1024bit-ECFP6 & GBM & 0.004 & 0.774 & 0.651 & 0.026 & 0.022 & 100.5 \\
\hline M0508 & 512 & $\exp 14$ & 1024bit-ECFP6 & GBM & 0.064 & 0.658 & 0.607 & 0.013 & 0.051 & 100.4 \\
\hline M0509 & 512 & exp15 & 1024bit-ECFP6 & GBM & 0.002 & 0.854 & 0.694 & 0.023 & 0.021 & 100.9 \\
\hline M0510 & 512 & exp16 & 1024bit-ECFP6 & GBM & 0.035 & 0.803 & 0.695 & 0.025 & 0.011 & 10000.9 \\
\hline M0511 & 512 & exp17 & 1024bit-ECFP6 & GBM & 0.001 & 0.858 & 0.704 & 0.036 & 0.035 & 1000.2 \\
\hline M0512 & 512 & $\exp 18$ & 1024bit-ECFP6 & GBM & 0.003 & 0.773 & 0.693 & 0.023 & 0.020 & 100.9 \\
\hline M0513 & 512 & $\exp 19$ & 1024bit-ECFP6 & GBM & 0.004 & 0.800 & 0.667 & 0.012 & 0.008 & 100.5 \\
\hline M0514 & 512 & exp01 & 1024bit-ECFP6 & $\mathrm{RF}$ & 0.039 & 0.693 & 0.629 & 0.006 & 0.033 & 700 \\
\hline M0515 & 512 & exp02 & 1024bit-ECFP6 & $\mathrm{RF}$ & 0.000 & 0.804 & 0.668 & 0.008 & 0.008 & 100 \\
\hline M0516 & 512 & exp03 & 1024bit-ECFP6 & $\mathrm{RF}$ & 0.043 & 0.673 & 0.627 & 0.005 & 0.038 & 10 \\
\hline M0517 & 512 & exp04 & 1024bit-ECFP6 & $\mathrm{RF}$ & 0.006 & 0.732 & 0.650 & 0.007 & 0.001 & 200 \\
\hline M0518 & 512 & exp05 & 1024bit-ECFP6 & $\mathrm{RF}$ & 0.011 & 0.724 & 0.670 & 0.025 & 0.014 & 100 \\
\hline M0519 & 512 & exp06 & 1024bit-ECFP6 & $\mathrm{RF}$ & 0.000 & 0.827 & 0.695 & 0.009 & 0.009 & 300 \\
\hline M0520 & 512 & exp07 & 1024bit-ECFP6 & $\mathrm{RF}$ & 0.007 & 0.765 & 0.665 & 0.007 & 0.000 & 50 \\
\hline M0521 & 512 & exp08 & 1024bit-ECFP6 & $\mathrm{RF}$ & 0.001 & 0.804 & 0.702 & 0.008 & 0.007 & 300 \\
\hline M0522 & 512 & exp09 & 1024bit-ECFP6 & $\mathrm{RF}$ & 0.036 & 0.687 & 0.633 & 0.022 & 0.014 & 300 \\
\hline M0523 & 512 & $\exp 10$ & 1024bit-ECFP6 & $\mathrm{RF}$ & 0.001 & 0.812 & 0.684 & 0.017 & 0.016 & 700 \\
\hline M0524 & 512 & $\exp 11$ & 1024bit-ECFP6 & $\mathrm{RF}$ & 0.035 & 0.916 & 0.726 & 0.006 & 0.029 & 300 \\
\hline M0525 & 512 & $\exp 12$ & 1024bit-ECFP6 & $\mathrm{RF}$ & 0.004 & 0.806 & 0.689 & 0.009 & 0.005 & 10 \\
\hline M0526 & 512 & exp13 & 1024bit-ECFP6 & $\mathrm{RF}$ & 0.000 & 0.805 & 0.677 & 0.007 & 0.007 & 50 \\
\hline M0527 & 512 & exp14 & 1024bit-ECFP6 & $\mathrm{RF}$ & 0.040 & 0.691 & 0.619 & 0.010 & 0.030 & 50 \\
\hline M0528 & 512 & exp15 & 1024bit-ECFP6 & $\mathrm{RF}$ & 0.000 & 0.819 & 0.688 & 0.006 & 0.006 & 700 \\
\hline M0529 & 512 & $\exp 16$ & 1024bit-ECFP6 & $\mathrm{RF}$ & 0.015 & 0.691 & 0.629 & 0.013 & 0.002 & 1000 \\
\hline M0530 & 512 & $\exp 17$ & 1024bit-ECFP6 & $\mathrm{RF}$ & 0.000 & 0.840 & 0.699 & 0.025 & 0.025 & 50 \\
\hline M0531 & 512 & $\exp 18$ & 1024bit-ECFP6 & $\mathrm{RF}$ & 0.003 & 0.762 & 0.692 & 0.004 & 0.001 & 10 \\
\hline M0532 & 512 & exp19 & 1024bit-ECFP6 & $\mathrm{RF}$ & 0.011 & 0.946 & 0.738 & 0.005 & 0.006 & 10 \\
\hline
\end{tabular}


Supporting Information (Texts S1-S9, Figures S1-S41 and Tables S1-S12)

\begin{tabular}{|c|c|c|c|c|c|c|c|c|c|c|}
\hline M0533 & 512 & exp01 & 2048bit-ECFP6 & KNN & 0.010 & 0.751 & 0.651 & 0.018 & 0.008 & 9 distance \\
\hline M0534 & 512 & exp02 & 2048bit-ECFP6 & KNN & 0.000 & 0.744 & 0.658 & 0.014 & 0.014 & 13 uniform \\
\hline M0535 & 512 & exp03 & 2048bit-ECFP6 & KNN & 0.063 & 0.762 & 0.665 & 0.006 & 0.057 & 1 distance \\
\hline M0536 & 512 & exp04 & 2048bit-ECFP6 & KNN & 0.005 & 0.966 & 0.762 & 0.016 & 0.011 & 1 distance \\
\hline M0537 & 512 & exp05 & 2048bit-ECFP6 & KNN & 0.005 & 0.731 & 0.664 & 0.032 & 0.027 & 11 distance \\
\hline M0538 & 512 & exp06 & 2048bit-ECFP6 & KNN & 0.001 & 0.809 & 0.677 & 0.025 & 0.024 & 5 uniform \\
\hline M0539 & 512 & exp07 & 2048bit-ECFP6 & KNN & 0.000 & 1.070 & 0.805 & 0.013 & 0.013 & 1 distance \\
\hline M0540 & 512 & exp08 & 2048bit-ECFP6 & KNN & 0.029 & 0.926 & 0.759 & 0.011 & 0.018 & 3 uniform \\
\hline M0541 & 512 & exp09 & 2048bit-ECFP6 & KNN & 0.013 & 0.738 & 0.654 & 0.026 & 0.013 & 3 uniform \\
\hline M0542 & 512 & exp10 & 2048bit-ECFP6 & KNN & 0.003 & 0.774 & 0.650 & 0.025 & 0.022 & 13 uniform \\
\hline M0543 & 512 & exp11 & 2048bit-ECFP6 & KNN & 0.018 & 0.776 & 0.652 & 0.011 & 0.006 & 11 uniform \\
\hline M0544 & 512 & $\exp 12$ & 2048bit-ECFP6 & KNN & 0.005 & 0.777 & 0.680 & 0.008 & 0.003 & 13 distance \\
\hline M0545 & 512 & exp13 & 2048bit-ECFP6 & KNN & 0.005 & 0.811 & 0.691 & 0.009 & 0.004 & 3 uniform \\
\hline M0546 & 512 & exp14 & 2048bit-ECFP6 & KNN & 0.040 & 0.679 & 0.608 & 0.030 & 0.010 & 5 uniform \\
\hline M0547 & 512 & $\exp 15$ & 2048bit-ECFP6 & KNN & 0.003 & 0.708 & 0.660 & 0.009 & 0.006 & 13 uniform \\
\hline M0548 & 512 & exp16 & 2048bit-ECFP6 & KNN & 0.010 & 0.769 & 0.655 & 0.015 & 0.005 & 3 distance \\
\hline M0549 & 512 & exp17 & 2048bit-ECFP6 & KNN & 0.002 & 0.932 & 0.734 & 0.029 & 0.027 & 3 distance \\
\hline M0550 & 512 & $\exp 18$ & 2048bit-ECFP6 & KNN & 0.004 & 0.680 & 0.624 & 0.017 & 0.013 & 15 uniform \\
\hline M0551 & 512 & $\exp 19$ & 2048bit-ECFP6 & KNN & 0.031 & 0.818 & 0.682 & 0.016 & 0.015 & 9 uniform \\
\hline M0552 & 512 & exp01 & 2048bit-ECFP6 & SVM & 0.010 & 1.520 & 0.961 & 0.017 & 0.007 & $100000.05 \mathrm{e}-05$ \\
\hline M0553 & 512 & exp02 & 2048bit-ECFP6 & SVM & 0.000 & 0.717 & 0.624 & 0.014 & 0.014 & $105 \mathrm{e}-05$ \\
\hline M0554 & 512 & exp03 & 2048bit-ECFP6 & SVM & 0.003 & 0.924 & 0.746 & 0.019 & 0.016 & $5000.05 \mathrm{e}-05$ \\
\hline M0555 & 512 & exp04 & 2048bit-ECFP6 & SVM & 0.005 & 1.729 & 1.037 & 0.014 & 0.009 & 100000.00 .0001 \\
\hline M0556 & 512 & $\exp 05$ & 2048bit-ECFP6 & SVM & 0.001 & 0.841 & 0.731 & 0.025 & 0.024 & 100.05 \\
\hline M0557 & 512 & exp06 & 2048bit-ECFP6 & SVM & 0.001 & 0.793 & 0.676 & 0.023 & 0.022 & $\begin{array}{ll}10.1 \\
\end{array}$ \\
\hline M0558 & 512 & exp07 & 2048bit-ECFP6 & SVM & 0.000 & 0.734 & 0.654 & 0.025 & 0.025 & $1000.01 \mathrm{e}-05$ \\
\hline M0559 & 512 & exp08 & 2048bit-ECFP6 & SVM & 0.003 & 0.701 & 0.640 & 0.011 & 0.008 & $5001 \mathrm{e}-05$ \\
\hline M0560 & 512 & exp09 & 2048bit-ECFP6 & SVM & 0.007 & 0.805 & 0.694 & 0.024 & 0.017 & 100.05 \\
\hline M0561 & 512 & exp10 & 2048bit-ECFP6 & SVM & 0.010 & 0.773 & 0.649 & 0.034 & 0.024 & $5001 \mathrm{e}-05$ \\
\hline M0562 & 512 & $\exp 11$ & 2048bit-ECFP6 & SVM & 0.035 & 1.025 & 0.778 & 0.020 & 0.015 & $10000.01 \mathrm{e}-05$ \\
\hline M0563 & 512 & $\exp 12$ & 2048bit-ECFP6 & SVM & 0.006 & 0.872 & 0.722 & 0.024 & 0.018 & 500.001 \\
\hline M0564 & 512 & exp13 & 2048bit-ECFP6 & SVM & 0.051 & 0.680 & 0.612 & 0.010 & 0.041 & 10.0001 \\
\hline M0565 & 512 & exp14 & 2048bit-ECFP6 & SVM & 0.058 & 1.215 & 0.890 & 0.023 & 0.035 & 100000.00 .0001 \\
\hline M0566 & 512 & exp15 & 2048bit-ECFP6 & SVM & 0.001 & 0.819 & 0.699 & 0.024 & 0.023 & $1000.05 \mathrm{e}-05$ \\
\hline M0567 & 512 & exp16 & 2048bit-ECFP6 & SVM & 0.014 & 0.744 & 0.651 & 0.023 & 0.009 & 50.05 \\
\hline M0568 & 512 & $\exp 17$ & 2048bit-ECFP6 & SVM & 0.001 & 0.868 & 0.711 & 0.036 & 0.035 & 100000.00 .05 \\
\hline M0569 & 512 & $\exp 18$ & 2048bit-ECFP6 & SVM & 0.018 & 1.391 & 0.955 & 0.017 & 0.001 & $100000.05 \mathrm{e}-05$ \\
\hline M0570 & 512 & exp19 & 2048bit-ECFP6 & SVM & 0.046 & 0.806 & 0.666 & 0.013 & 0.033 & $5001 \mathrm{e}-05$ \\
\hline M0571 & 512 & exp01 & 2048bit-ECFP6 & GBM & 0.005 & 0.690 & 0.614 & 0.021 & 0.016 & 100.1 \\
\hline M0572 & 512 & exp02 & 2048bit-ECFP6 & GBM & 0.000 & 0.977 & 0.752 & 0.018 & 0.018 & 10000.5 \\
\hline M0573 & 512 & exp03 & 2048bit-ECFP6 & GBM & 0.020 & 0.691 & 0.641 & 0.011 & 0.009 & 100.9 \\
\hline M0574 & 512 & exp04 & 2048bit-ECFP6 & GBM & 0.044 & 0.626 & 0.599 & 0.012 & 0.032 & 100.3 \\
\hline M0575 & 512 & $\exp 05$ & 2048bit-ECFP6 & GBM & 0.000 & 0.906 & 0.747 & 0.046 & 0.046 & 500.6 \\
\hline M0576 & 512 & exp06 & 2048bit-ECFP6 & GBM & 0.007 & 1.183 & 0.796 & 0.016 & 0.009 & 500.8 \\
\hline M0577 & 512 & exp07 & 2048bit-ECFP6 & GBM & 0.003 & 0.684 & 0.612 & 0.018 & 0.015 & 100.1 \\
\hline
\end{tabular}


Supporting Information (Texts S1-S9, Figures S1-S41 and Tables S1-S12)

\begin{tabular}{|c|c|c|c|c|c|c|c|c|c|c|}
\hline M0578 & 512 & exp08 & 2048bit-ECFP6 & GBM & 0.006 & 0.689 & 0.628 & 0.023 & 0.017 & 100.4 \\
\hline M0579 & 512 & exp09 & 2048bit-ECFP6 & GBM & 0.009 & 0.922 & 0.738 & 0.019 & 0.011 & 9000.2 \\
\hline M0580 & 512 & exp10 & 2048bit-ECFP6 & GBM & 0.004 & 0.901 & 0.727 & 0.042 & 0.038 & 3000.1 \\
\hline M0581 & 512 & exp11 & 2048bit-ECFP6 & GBM & 0.044 & 0.910 & 0.707 & 0.013 & 0.031 & 2000.1 \\
\hline M0582 & 512 & exp12 & 2048bit-ECFP6 & GBM & 0.000 & 0.663 & 0.609 & 0.020 & 0.020 & 100.1 \\
\hline M0583 & 512 & exp13 & 2048bit-ECFP6 & GBM & 0.005 & 0.835 & 0.699 & 0.009 & 0.004 & 100.8 \\
\hline M0584 & 512 & exp14 & 2048bit-ECFP6 & GBM & 0.041 & 0.675 & 0.609 & 0.016 & 0.025 & 100.6 \\
\hline M0585 & 512 & $\exp 15$ & 2048bit-ECFP6 & GBM & 0.000 & 1.017 & 0.763 & 0.017 & 0.017 & 2000.7 \\
\hline M0586 & 512 & exp16 & 2048bit-ECFP6 & GBM & 0.011 & 0.743 & 0.654 & 0.027 & 0.016 & 100.7 \\
\hline M0587 & 512 & exp17 & 2048bit-ECFP6 & GBM & 0.000 & 1.002 & 0.762 & 0.032 & 0.032 & 10000.7 \\
\hline M0588 & 512 & exp18 & 2048bit-ECFP6 & GBM & 0.003 & 0.667 & 0.607 & 0.036 & 0.033 & 100.1 \\
\hline M0589 & 512 & exp19 & 2048bit-ECFP6 & GBM & 0.016 & 1.269 & 0.822 & 0.011 & 0.005 & 500.7 \\
\hline M0590 & 512 & exp01 & 2048bit-ECFP6 & $\mathrm{RF}$ & 0.015 & 0.761 & 0.673 & 0.012 & 0.003 & 10 \\
\hline M0591 & 512 & exp02 & 2048bit-ECFP6 & $\mathrm{RF}$ & 0.001 & 0.791 & 0.664 & 0.009 & 0.008 & 300 \\
\hline M0592 & 512 & exp03 & 2048bit-ECFP6 & $\mathrm{RF}$ & 0.031 & 0.692 & 0.640 & 0.004 & 0.027 & 10 \\
\hline M0593 & 512 & exp04 & 2048bit-ECFP6 & $\mathrm{RF}$ & 0.008 & 0.720 & 0.648 & 0.010 & 0.002 & 200 \\
\hline M0594 & 512 & exp05 & 2048bit-ECFP6 & $\mathrm{RF}$ & 0.007 & 0.789 & 0.702 & 0.035 & 0.028 & 10 \\
\hline M0595 & 512 & exp06 & 2048bit-ECFP6 & $\mathrm{RF}$ & 0.006 & 0.980 & 0.743 & 0.010 & 0.004 & 400 \\
\hline M0596 & 512 & exp07 & 2048bit-ECFP6 & $\mathrm{RF}$ & 0.004 & 0.783 & 0.672 & 0.008 & 0.004 & 800 \\
\hline M0597 & 512 & exp08 & 2048bit-ECFP6 & $\mathrm{RF}$ & 0.000 & 0.806 & 0.713 & 0.008 & 0.008 & 800 \\
\hline M0598 & 512 & exp09 & 2048bit-ECFP6 & $\mathrm{RF}$ & 0.025 & 0.708 & 0.646 & 0.024 & 0.001 & 300 \\
\hline M0599 & 512 & exp10 & 2048bit-ECFP6 & $\mathrm{RF}$ & 0.003 & 0.841 & 0.703 & 0.018 & 0.015 & 600 \\
\hline M0600 & 512 & exp11 & 2048bit-ECFP6 & $\mathrm{RF}$ & 0.033 & 0.916 & 0.729 & 0.007 & 0.026 & 300 \\
\hline M0601 & 512 & $\exp 12$ & 2048bit-ECFP6 & $\mathrm{RF}$ & 0.003 & 0.797 & 0.688 & 0.008 & 0.005 & 50 \\
\hline M0602 & 512 & $\exp 13$ & 2048bit-ECFP6 & $\mathrm{RF}$ & 0.000 & 0.815 & 0.696 & 0.009 & 0.009 & 10 \\
\hline M0603 & 512 & $\exp 14$ & 2048bit-ECFP6 & $\mathrm{RF}$ & 0.032 & 0.709 & 0.634 & 0.011 & 0.021 & 500 \\
\hline M0604 & 512 & $\exp 15$ & 2048bit-ECFP6 & $\mathrm{RF}$ & 0.000 & 0.837 & 0.708 & 0.007 & 0.007 & 1000 \\
\hline M0605 & 512 & exp16 & 2048bit-ECFP6 & $\mathrm{RF}$ & 0.018 & 0.692 & 0.639 & 0.024 & 0.006 & 100 \\
\hline M0606 & 512 & exp17 & 2048bit-ECFP6 & $\mathrm{RF}$ & 0.001 & 0.849 & 0.694 & 0.032 & 0.031 & 200 \\
\hline M0607 & 512 & $\exp 18$ & 2048bit-ECFP6 & $\mathrm{RF}$ & 0.002 & 0.780 & 0.701 & 0.003 & 0.001 & 600 \\
\hline M0608 & 512 & exp19 & 2048bit-ECFP6 & $\mathrm{RF}$ & 0.022 & 0.982 & 0.740 & 0.004 & 0.018 & 100 \\
\hline M0609 & 256 & exp01 & 1024bit-ECFP4 & KNN & 0.011 & 0.770 & 0.655 & 0.022 & 0.011 & 7 distance \\
\hline M0610 & 256 & exp02 & 1024bit-ECFP4 & KNN & 0.002 & 0.826 & 0.700 & 0.012 & 0.010 & 11 distance \\
\hline M0611 & 256 & exp03 & 1024bit-ECFP4 & KNN & 0.032 & 0.687 & 0.653 & 0.011 & 0.021 & 3 uniform \\
\hline M0612 & 256 & $\exp 04$ & 1024bit-ECFP4 & KNN & 0.004 & 0.779 & 0.667 & 0.017 & 0.013 & 5 distance \\
\hline M0613 & 256 & exp05 & 1024bit-ECFP4 & KNN & 0.001 & 0.778 & 0.681 & 0.031 & 0.030 & 11 distance \\
\hline M0614 & 256 & exp06 & 1024bit-ECFP4 & KNN & 0.000 & 0.814 & 0.692 & 0.026 & 0.026 & 13 distance \\
\hline M0615 & 256 & exp07 & 1024bit-ECFP4 & KNN & 0.000 & 0.801 & 0.685 & 0.010 & 0.010 & 3 uniform \\
\hline M0616 & 256 & exp08 & 1024bit-ECFP4 & KNN & 0.001 & 0.830 & 0.712 & 0.010 & 0.009 & 15 distance \\
\hline M0617 & 256 & exp09 & 1024bit-ECFP4 & KNN & 0.025 & 0.769 & 0.677 & 0.027 & 0.002 & 3 distance \\
\hline M0618 & 256 & exp10 & 1024bit-ECFP4 & KNN & 0.003 & 0.781 & 0.663 & 0.020 & 0.017 & 11 uniform \\
\hline M0619 & 256 & $\exp 11$ & 1024bit-ECFP4 & KNN & 0.053 & 0.795 & 0.665 & 0.009 & 0.044 & 13 uniform \\
\hline M0620 & 256 & $\exp 12$ & 1024bit-ECFP4 & KNN & 0.021 & 0.830 & 0.704 & 0.010 & 0.011 & 13 distance \\
\hline M0621 & 256 & $\exp 13$ & 1024bit-ECFP4 & KNN & 0.002 & 0.709 & 0.614 & 0.017 & 0.015 & 15 uniform \\
\hline M0622 & 256 & exp14 & 1024bit-ECFP4 & KNN & 0.025 & 0.696 & 0.618 & 0.020 & 0.005 & 5 uniform \\
\hline
\end{tabular}


Supporting Information (Texts S1-S9, Figures S1-S41 and Tables S1-S12)

\begin{tabular}{|c|c|c|c|c|c|c|c|c|c|c|}
\hline M0623 & 256 & exp15 & 1024bit-ECFP4 & KNN & 0.003 & 0.718 & 0.660 & 0.010 & 0.007 & 9 uniform \\
\hline M0624 & 256 & exp16 & 1024bit-ECFP4 & KNN & 0.002 & 1.113 & 0.808 & 0.026 & 0.024 & 1 distance \\
\hline M0625 & 256 & exp17 & 1024bit-ECFP4 & KNN & 0.000 & 0.894 & 0.722 & 0.031 & 0.031 & 7 distance \\
\hline M0626 & 256 & exp18 & 1024bit-ECFP4 & KNN & 0.008 & 0.675 & 0.627 & 0.011 & 0.003 & 15 uniform \\
\hline M0627 & 256 & exp19 & 1024bit-ECFP4 & KNN & 0.015 & 0.825 & 0.681 & 0.015 & 0.000 & 7 uniform \\
\hline M0628 & 256 & exp01 & 1024bit-ECFP4 & SVM & 0.022 & 0.698 & 0.632 & 0.012 & 0.010 & 10.1 \\
\hline M0629 & 256 & $\operatorname{exp02}$ & 1024bit-ECFP4 & SVM & 0.010 & 1.327 & 0.893 & 0.027 & 0.017 & $100000.05 \mathrm{e}-05$ \\
\hline M0630 & 256 & exp03 & 1024bit-ECFP4 & SVM & 0.012 & 1.041 & 0.750 & 0.027 & 0.015 & 100000.00 .0001 \\
\hline M0631 & 256 & exp04 & 1024bit-ECFP4 & SVM & 0.005 & 0.770 & 0.672 & 0.015 & 0.010 & 100.1 \\
\hline M0632 & 256 & exp05 & 1024bit-ECFP4 & SVM & 0.001 & 0.851 & 0.736 & 0.015 & 0.014 & 50.1 \\
\hline M0633 & 256 & exp06 & 1024bit-ECFP4 & SVM & 0.002 & 0.835 & 0.701 & 0.029 & 0.027 & 50.01 \\
\hline M0634 & 256 & $\exp 07$ & 1024bit-ECFP4 & SVM & 0.001 & 0.733 & 0.648 & 0.026 & 0.025 & $1000.01 \mathrm{e}-05$ \\
\hline M0635 & 256 & exp08 & 1024bit-ECFP4 & SVM & 0.001 & 1.146 & 0.832 & 0.011 & 0.010 & $100000.05 \mathrm{e}-05$ \\
\hline M0636 & 256 & exp09 & 1024bit-ECFP4 & SVM & 0.008 & 0.868 & 0.708 & 0.030 & 0.022 & 5000.00 .05 \\
\hline M0637 & 256 & exp10 & 1024bit-ECFP4 & SVM & 0.012 & 1.193 & 0.847 & 0.056 & 0.044 & 1000.00 .001 \\
\hline M0638 & 256 & exp11 & 1024bit-ECFP4 & SVM & 0.001 & 0.758 & 0.656 & 0.017 & 0.015 & 50.005 \\
\hline M0639 & 256 & exp12 & 1024bit-ECFP4 & SVM & 0.004 & 0.756 & 0.657 & 0.024 & 0.020 & 10.05 \\
\hline M0640 & 256 & exp13 & 1024bit-ECFP4 & SVM & 0.052 & 0.676 & 0.610 & 0.012 & 0.040 & $55 \mathrm{e}-05$ \\
\hline M0641 & 256 & exp14 & 1024bit-ECFP4 & SVM & 0.037 & 0.680 & 0.596 & 0.017 & 0.020 & 10.05 \\
\hline M0642 & 256 & exp15 & 1024bit-ECFP4 & SVM & 0.001 & 1.020 & 0.803 & 0.010 & 0.009 & 1000.01 \\
\hline M0643 & 256 & exp16 & 1024bit-ECFP4 & SVM & 0.005 & 0.771 & 0.665 & 0.027 & 0.022 & 50.1 \\
\hline M0644 & 256 & exp17 & 1024bit-ECFP4 & SVM & 0.002 & 1.145 & 0.852 & 0.035 & 0.033 & 100000.00 .01 \\
\hline M0645 & 256 & exp18 & 1024bit-ECFP4 & SVM & 0.006 & 1.014 & 0.798 & 0.014 & 0.008 & $100000.05 \mathrm{e}-05$ \\
\hline M0646 & 256 & exp19 & 1024bit-ECFP4 & SVM & 0.105 & 0.743 & 0.646 & 0.009 & 0.096 & $1001 \mathrm{e}-05$ \\
\hline M0647 & 256 & exp01 & 1024bit-ECFP4 & GBM & 0.026 & 0.715 & 0.618 & 0.015 & 0.011 & 100.8 \\
\hline M0648 & 256 & exp02 & 1024bit-ECFP4 & GBM & 0.003 & 0.843 & 0.694 & 0.015 & 0.011 & 100.9 \\
\hline M0649 & 256 & exp03 & 1024bit-ECFP4 & GBM & 0.055 & 0.699 & 0.661 & 0.021 & 0.035 & 1000.6 \\
\hline M0650 & 256 & exp04 & 1024bit-ECFP4 & GBM & 0.003 & 0.910 & 0.718 & 0.015 & 0.012 & 8000.3 \\
\hline M0651 & 256 & exp05 & 1024bit-ECFP4 & GBM & 0.005 & 0.935 & 0.756 & 0.014 & 0.009 & 4000.3 \\
\hline M0652 & 256 & exp06 & 1024bit-ECFP4 & GBM & 0.004 & 0.973 & 0.756 & 0.026 & 0.022 & 1000.4 \\
\hline M0653 & 256 & exp07 & 1024bit-ECFP4 & GBM & 0.001 & 0.878 & 0.728 & 0.015 & 0.014 & 500.6 \\
\hline M0654 & 256 & exp08 & 1024bit-ECFP4 & GBM & 0.004 & 0.699 & 0.635 & 0.019 & 0.015 & 100.3 \\
\hline M0655 & 256 & exp09 & 1024bit-ECFP4 & GBM & 0.014 & 0.918 & 0.734 & 0.035 & 0.021 & 7000.4 \\
\hline M0656 & 256 & exp10 & 1024bit-ECFP4 & GBM & 0.003 & 0.930 & 0.733 & 0.067 & 0.064 & 1000.4 \\
\hline M0657 & 256 & exp11 & 1024bit-ECFP4 & GBM & 0.035 & 0.979 & 0.733 & 0.010 & 0.025 & 1000.3 \\
\hline M0658 & 256 & exp12 & 1024bit-ECFP4 & GBM & 0.013 & 1.092 & 0.827 & 0.021 & 0.008 & 2000.6 \\
\hline M0659 & 256 & exp13 & 1024bit-ECFP4 & GBM & 0.000 & 0.718 & 0.637 & 0.012 & 0.012 & 500.1 \\
\hline M0660 & 256 & exp14 & 1024bit-ECFP4 & GBM & 0.019 & 0.932 & 0.754 & 0.014 & 0.005 & 10000.4 \\
\hline M0661 & 256 & exp15 & 1024bit-ECFP4 & GBM & 0.001 & 0.935 & 0.750 & 0.026 & 0.025 & 500.9 \\
\hline M0662 & 256 & exp16 & 1024bit-ECFP4 & GBM & 0.002 & 1.038 & 0.785 & 0.026 & 0.024 & 2000.9 \\
\hline M0663 & 256 & exp17 & 1024bit-ECFP4 & GBM & 0.008 & 0.907 & 0.715 & 0.026 & 0.018 & 1000.2 \\
\hline M0664 & 256 & exp18 & 1024bit-ECFP4 & GBM & 0.012 & 0.872 & 0.730 & 0.015 & 0.003 & 1000.8 \\
\hline M0665 & 256 & exp19 & 1024bit-ECFP4 & GBM & 0.007 & 1.019 & 0.757 & 0.021 & 0.014 & 100.9 \\
\hline M0666 & 256 & exp01 & 1024bit-ECFP4 & $\mathrm{RF}$ & 0.049 & 0.694 & 0.619 & 0.008 & 0.041 & 700 \\
\hline M0667 & 256 & $\exp 02$ & 1024bit-ECFP4 & $\mathrm{RF}$ & 0.000 & 0.829 & 0.682 & 0.008 & 0.008 & 300 \\
\hline
\end{tabular}


Supporting Information (Texts S1-S9, Figures S1-S41 and Tables S1-S12)

\begin{tabular}{|c|c|c|c|c|c|c|c|c|c|c|}
\hline M0668 & 256 & exp03 & 1024bit-ECFP4 & $\mathrm{RF}$ & 0.050 & 0.671 & 0.641 & 0.005 & 0.045 & 10 \\
\hline M0669 & 256 & exp04 & 1024bit-ECFP4 & $\mathrm{RF}$ & 0.005 & 0.746 & 0.664 & 0.013 & 0.008 & 200 \\
\hline M0670 & 256 & exp05 & 1024bit-ECFP4 & $\mathrm{RF}$ & 0.008 & 0.752 & 0.688 & 0.016 & 0.008 & 200 \\
\hline M0671 & 256 & exp06 & 1024bit-ECFP4 & $\mathrm{RF}$ & 0.002 & 0.909 & 0.736 & 0.011 & 0.009 & 10 \\
\hline M0672 & 256 & exp07 & 1024bit-ECFP4 & $\mathrm{RF}$ & 0.013 & 0.765 & 0.671 & 0.008 & 0.005 & 50 \\
\hline M0673 & 256 & exp08 & 1024bit-ECFP4 & $\mathrm{RF}$ & 0.001 & 0.812 & 0.703 & 0.004 & 0.003 & 100 \\
\hline M0674 & 256 & exp09 & 1024bit-ECFP4 & $\mathrm{RF}$ & 0.034 & 0.685 & 0.621 & 0.034 & 0.000 & 600 \\
\hline M0675 & 256 & $\exp 10$ & 1024bit-ECFP4 & $\mathrm{RF}$ & 0.005 & 0.877 & 0.718 & 0.036 & 0.031 & 100 \\
\hline M0676 & 256 & exp11 & 1024bit-ECFP4 & $\mathrm{RF}$ & 0.032 & 0.961 & 0.747 & 0.002 & 0.030 & 50 \\
\hline M0677 & 256 & exp12 & 1024bit-ECFP4 & $\mathrm{RF}$ & 0.013 & 0.849 & 0.717 & 0.011 & 0.002 & 100 \\
\hline M0678 & 256 & exp13 & 1024bit-ECFP4 & $\mathrm{RF}$ & 0.002 & 0.811 & 0.685 & 0.006 & 0.004 & 10 \\
\hline M0679 & 256 & exp14 & 1024bit-ECFP4 & $\mathrm{RF}$ & 0.039 & 0.706 & 0.631 & 0.009 & 0.030 & 300 \\
\hline M0680 & 256 & exp15 & 1024bit-ECFP4 & $\mathrm{RF}$ & 0.004 & 0.874 & 0.726 & 0.008 & 0.004 & 1000 \\
\hline M0681 & 256 & exp16 & 1024bit-ECFP4 & $\mathrm{RF}$ & 0.001 & 0.760 & 0.661 & 0.023 & 0.022 & 500 \\
\hline M0682 & 256 & exp17 & 1024bit-ECFP4 & $\mathrm{RF}$ & 0.001 & 0.881 & 0.721 & 0.020 & 0.019 & 200 \\
\hline M0683 & 256 & exp18 & 1024bit-ECFP4 & $\mathrm{RF}$ & 0.015 & 0.728 & 0.666 & 0.005 & 0.010 & 10 \\
\hline M0684 & 256 & exp19 & 1024bit-ECFP4 & $\mathrm{RF}$ & 0.020 & 1.020 & 0.744 & 0.010 & 0.010 & 10 \\
\hline M0685 & 256 & exp01 & 2048bit-ECFP4 & KNN & 0.011 & 0.756 & 0.646 & 0.019 & 0.008 & 11 distance \\
\hline M0686 & 256 & $\exp 02$ & 2048bit-ECFP4 & KNN & 0.000 & 0.850 & 0.713 & 0.009 & 0.009 & 15 distance \\
\hline M0687 & 256 & exp03 & 2048bit-ECFP4 & KNN & 0.025 & 0.694 & 0.657 & 0.012 & 0.013 & 3 uniform \\
\hline M0688 & 256 & exp04 & 2048bit-ECFP4 & KNN & 0.004 & 0.804 & 0.682 & 0.016 & 0.012 & 3 distance \\
\hline M0689 & 256 & exp05 & 2048bit-ECFP4 & KNN & 0.000 & 0.781 & 0.689 & 0.030 & 0.030 & 13 distance \\
\hline M0690 & 256 & exp06 & 2048bit-ECFP4 & KNN & 0.000 & 0.871 & 0.712 & 0.024 & 0.024 & 7 distance \\
\hline M0691 & 256 & exp07 & 2048bit-ECFP4 & KNN & 0.001 & 1.044 & 0.794 & 0.015 & 0.014 & 1 uniform \\
\hline M0692 & 256 & exp08 & 2048bit-ECFP4 & KNN & 0.004 & 0.858 & 0.720 & 0.012 & 0.008 & 13 distance \\
\hline M0693 & 256 & exp09 & 2048bit-ECFP4 & KNN & 0.015 & 0.695 & 0.633 & 0.026 & 0.011 & 5 uniform \\
\hline M0694 & 256 & $\exp 10$ & 2048bit-ECFP4 & KNN & 0.007 & 0.716 & 0.633 & 0.028 & 0.021 & 13 uniform \\
\hline M0695 & 256 & exp11 & 2048bit-ECFP4 & KNN & 0.027 & 0.767 & 0.648 & 0.010 & 0.017 & 15 uniform \\
\hline M0696 & 256 & exp12 & 2048bit-ECFP4 & KNN & 0.000 & 1.144 & 0.799 & 0.009 & 0.009 & 1 uniform \\
\hline M0697 & 256 & exp13 & 2048bit-ECFP4 & KNN & 0.000 & 0.724 & 0.628 & 0.013 & 0.013 & 15 uniform \\
\hline M0698 & 256 & exp14 & 2048bit-ECFP4 & KNN & 0.035 & 0.714 & 0.618 & 0.019 & 0.016 & 3 uniform \\
\hline M0699 & 256 & exp15 & 2048bit-ECFP4 & KNN & 0.005 & 0.701 & 0.648 & 0.015 & 0.010 & 15 uniform \\
\hline M0700 & 256 & exp16 & 2048bit-ECFP4 & KNN & 0.002 & 0.765 & 0.673 & 0.014 & 0.012 & 7 distance \\
\hline M0701 & 256 & exp17 & 2048bit-ECFP4 & KNN & 0.000 & 0.915 & 0.733 & 0.029 & 0.029 & 9 distance \\
\hline M0702 & 256 & exp18 & 2048bit-ECFP4 & KNN & 0.006 & 0.677 & 0.625 & 0.012 & 0.006 & 13 uniform \\
\hline M0703 & 256 & exp19 & 2048bit-ECFP4 & KNN & 0.008 & 1.392 & 0.883 & 0.014 & 0.006 & 1 uniform \\
\hline M0704 & 256 & $\exp 01$ & 2048bit-ECFP4 & SVM & 0.032 & 0.694 & 0.616 & 0.013 & 0.019 & $11 \mathrm{e}-05$ \\
\hline M0705 & 256 & exp02 & 2048bit-ECFP4 & SVM & 0.009 & 1.335 & 0.862 & 0.011 & 0.002 & 50000.00 .0001 \\
\hline M0706 & 256 & exp03 & 2048bit-ECFP4 & SVM & 0.008 & 0.730 & 0.657 & 0.023 & 0.015 & $10000.01 \mathrm{e}-05$ \\
\hline M0707 & 256 & exp04 & 2048bit-ECFP4 & SVM & 0.029 & 0.900 & 0.763 & 0.020 & 0.009 & $100000.05 \mathrm{e}-05$ \\
\hline M0708 & 256 & exp05 & 2048bit-ECFP4 & SVM & 0.000 & 0.873 & 0.745 & 0.017 & 0.017 & 50.1 \\
\hline M0709 & 256 & exp06 & 2048bit-ECFP4 & SVM & 0.011 & 0.856 & 0.707 & 0.044 & 0.034 & $5000.01 \mathrm{e}-05$ \\
\hline M0710 & 256 & exp07 & 2048bit-ECFP4 & SVM & 0.000 & 0.713 & 0.638 & 0.024 & 0.024 & $1000.01 \mathrm{e}-05$ \\
\hline M0711 & 256 & exp08 & 2048bit-ECFP4 & SVM & 0.013 & 0.686 & 0.627 & 0.014 & 0.001 & $505 \mathrm{e}-05$ \\
\hline M0712 & 256 & exp09 & 2048bit-ECFP4 & SVM & 0.009 & 0.827 & 0.690 & 0.029 & 0.020 & 500.1 \\
\hline
\end{tabular}


Supporting Information (Texts S1-S9, Figures S1-S41 and Tables S1-S12)

\begin{tabular}{|c|c|c|c|c|c|c|c|c|c|c|}
\hline M0713 & 256 & exp10 & 2048bit-ECFP4 & SVM & 0.008 & 0.811 & 0.663 & 0.046 & 0.038 & 500.0005 \\
\hline M0714 & 256 & exp11 & 2048bit-ECFP4 & SVM & 0.028 & 1.221 & 0.865 & 0.020 & 0.008 & 50000.00 .0001 \\
\hline M0715 & 256 & exp12 & 2048bit-ECFP4 & SVM & 0.006 & 0.758 & 0.653 & 0.019 & 0.013 & 10.05 \\
\hline M0716 & 256 & exp13 & 2048bit-ECFP4 & SVM & 0.063 & 0.676 & 0.610 & 0.011 & 0.052 & $55 \mathrm{e}-05$ \\
\hline M0717 & 256 & exp14 & 2048bit-ECFP4 & SVM & 0.017 & 1.190 & 0.852 & 0.019 & 0.002 & 50000.00 .001 \\
\hline M0718 & 256 & exp15 & 2048bit-ECFP4 & SVM & 0.007 & 1.227 & 0.859 & 0.020 & 0.013 & 50000.00 .0005 \\
\hline M0719 & 256 & exp16 & 2048bit-ECFP4 & SVM & 0.002 & 0.714 & 0.638 & 0.020 & 0.018 & 10.1 \\
\hline M0720 & 256 & exp17 & 2048bit-ECFP4 & SVM & 0.000 & 1.032 & 0.798 & 0.043 & 0.043 & 5000.01 \\
\hline M0721 & 256 & exp18 & 2048bit-ECFP4 & SVM & 0.143 & 0.667 & 0.611 & 0.009 & 0.134 & $11 \mathrm{e}-05$ \\
\hline M0722 & 256 & exp19 & 2048bit-ECFP4 & SVM & 0.019 & 1.536 & 0.930 & 0.010 & 0.009 & 100000.00 .0001 \\
\hline M0723 & 256 & exp01 & 2048bit-ECFP4 & GBM & 0.047 & 0.808 & 0.695 & 0.023 & 0.024 & 9000.4 \\
\hline M0724 & 256 & $\exp 02$ & 2048bit-ECFP4 & GBM & 0.007 & 0.842 & 0.687 & 0.009 & 0.002 & 100.8 \\
\hline M0725 & 256 & exp03 & 2048bit-ECFP4 & GBM & 0.041 & 0.660 & 0.617 & 0.019 & 0.022 & 100.7 \\
\hline M0726 & 256 & exp04 & 2048bit-ECFP4 & GBM & 0.008 & 0.807 & 0.699 & 0.017 & 0.009 & 500.8 \\
\hline M0727 & 256 & exp05 & 2048bit-ECFP4 & GBM & 0.000 & 1.036 & 0.786 & 0.017 & 0.017 & 2000.8 \\
\hline M0728 & 256 & exp06 & 2048bit-ECFP4 & GBM & 0.007 & 0.988 & 0.771 & 0.023 & 0.016 & 5000.1 \\
\hline M0729 & 256 & exp07 & 2048bit-ECFP4 & GBM & 0.015 & 0.679 & 0.615 & 0.027 & 0.012 & 100.1 \\
\hline M0730 & 256 & exp08 & 2048bit-ECFP4 & GBM & 0.001 & 0.682 & 0.620 & 0.026 & 0.025 & 100.1 \\
\hline M0731 & 256 & exp09 & 2048bit-ECFP4 & GBM & 0.023 & 0.903 & 0.737 & 0.033 & 0.010 & 10000.8 \\
\hline M0732 & 256 & exp10 & 2048bit-ECFP4 & GBM & 0.009 & 0.846 & 0.675 & 0.056 & 0.047 & 500.3 \\
\hline M0733 & 256 & exp11 & 2048bit-ECFP4 & GBM & 0.021 & 0.895 & 0.715 & 0.017 & 0.004 & 1000.2 \\
\hline M0734 & 256 & exp12 & 2048bit-ECFP4 & GBM & 0.010 & 0.700 & 0.627 & 0.014 & 0.004 & 100.3 \\
\hline M0735 & 256 & exp13 & 2048bit-ECFP4 & GBM & 0.003 & 0.686 & 0.617 & 0.020 & 0.017 & 100.1 \\
\hline M0736 & 256 & exp14 & 2048bit-ECFP4 & GBM & 0.027 & 0.941 & 0.737 & 0.010 & 0.017 & 10000.9 \\
\hline M0737 & 256 & exp15 & 2048bit-ECFP4 & GBM & 0.009 & 0.837 & 0.702 & 0.012 & 0.003 & 1000.1 \\
\hline M0738 & 256 & exp16 & 2048bit-ECFP4 & GBM & 0.006 & 0.681 & 0.623 & 0.032 & 0.026 & 100.2 \\
\hline M0739 & 256 & exp17 & 2048bit-ECFP4 & GBM & 0.009 & 1.149 & 0.812 & 0.035 & 0.026 & 2000.9 \\
\hline M0740 & 256 & exp18 & 2048bit-ECFP4 & GBM & 0.023 & 0.689 & 0.643 & 0.021 & 0.002 & 100.7 \\
\hline M0741 & 256 & exp19 & 2048bit-ECFP4 & GBM & 0.009 & 0.783 & 0.662 & 0.016 & 0.007 & 100.3 \\
\hline M0742 & 256 & exp01 & 2048bit-ECFP4 & $\mathrm{RF}$ & 0.060 & 0.675 & 0.610 & 0.006 & 0.054 & 1000 \\
\hline M0743 & 256 & exp02 & 2048bit-ECFP4 & $\mathrm{RF}$ & 0.000 & 0.846 & 0.703 & 0.006 & 0.006 & 10 \\
\hline M0744 & 256 & exp03 & 2048bit-ECFP4 & $\mathrm{RF}$ & 0.066 & 0.668 & 0.638 & 0.005 & 0.061 & 10 \\
\hline M0745 & 256 & exp04 & 2048bit-ECFP4 & $\mathrm{RF}$ & 0.011 & 0.725 & 0.659 & 0.007 & 0.004 & 10 \\
\hline M0746 & 256 & exp05 & 2048bit-ECFP4 & $\mathrm{RF}$ & 0.000 & 0.811 & 0.713 & 0.017 & 0.017 & 100 \\
\hline M0747 & 256 & exp06 & 2048bit-ECFP4 & $\mathrm{RF}$ & 0.000 & 0.923 & 0.743 & 0.018 & 0.018 & 10 \\
\hline M0748 & 256 & exp07 & 2048bit-ECFP4 & $\mathrm{RF}$ & 0.003 & 0.850 & 0.705 & 0.013 & 0.010 & 10 \\
\hline M0749 & 256 & exp08 & 2048bit-ECFP4 & $\mathrm{RF}$ & 0.000 & 0.825 & 0.716 & 0.005 & 0.005 & 50 \\
\hline M0750 & 256 & exp09 & 2048bit-ECFP4 & $\mathrm{RF}$ & 0.042 & 0.686 & 0.634 & 0.030 & 0.012 & 10 \\
\hline M0751 & 256 & exp10 & 2048bit-ECFP4 & $\mathrm{RF}$ & 0.005 & 0.863 & 0.703 & 0.024 & 0.019 & 1000 \\
\hline M0752 & 256 & exp11 & 2048bit-ECFP4 & $\mathrm{RF}$ & 0.016 & 0.996 & 0.767 & 0.007 & 0.009 & 10 \\
\hline M0753 & 256 & exp12 & 2048bit-ECFP4 & $\mathrm{RF}$ & 0.010 & 0.848 & 0.709 & 0.010 & 0.000 & 50 \\
\hline M0754 & 256 & exp13 & 2048bit-ECFP4 & $\mathrm{RF}$ & 0.000 & 0.819 & 0.684 & 0.004 & 0.004 & 1000 \\
\hline M0755 & 256 & exp14 & 2048bit-ECFP4 & $\mathrm{RF}$ & 0.042 & 0.712 & 0.636 & 0.007 & 0.035 & 10 \\
\hline M0756 & 256 & exp15 & 2048bit-ECFP4 & $\mathrm{RF}$ & 0.002 & 0.868 & 0.727 & 0.005 & 0.003 & 800 \\
\hline M0757 & 256 & exp16 & 2048bit-ECFP4 & $\mathrm{RF}$ & 0.001 & 0.785 & 0.672 & 0.011 & 0.010 & 700 \\
\hline
\end{tabular}


Supporting Information (Texts S1-S9, Figures S1-S41 and Tables S1-S12)

\begin{tabular}{|c|c|c|c|c|c|c|c|c|c|c|}
\hline M0758 & 256 & $\exp 17$ & 2048bit-ECFP4 & $\mathrm{RF}$ & 0.006 & 0.902 & 0.725 & 0.027 & 0.021 & 1000 \\
\hline M0759 & 256 & $\exp 18$ & 2048bit-ECFP4 & $\mathrm{RF}$ & 0.012 & 0.743 & 0.679 & 0.008 & 0.004 & 10 \\
\hline M0760 & 256 & exp19 & 2048bit-ECFP4 & $\mathrm{RF}$ & 0.040 & 1.074 & 0.780 & 0.007 & 0.033 & 10 \\
\hline M0761 & 256 & exp01 & 1024bit-ECFP6 & KNN & 0.005 & 0.781 & 0.671 & 0.018 & 0.013 & 7 distance \\
\hline M0762 & 256 & exp02 & 1024bit-ECFP6 & KNN & 0.005 & 0.790 & 0.676 & 0.011 & 0.006 & 5 distance \\
\hline M0763 & 256 & exp03 & 1024bit-ECFP6 & KNN & 0.083 & 0.739 & 0.665 & 0.007 & 0.076 & 1 distance \\
\hline M0764 & 256 & exp04 & 1024bit-ECFP6 & KNN & 0.001 & 0.672 & 0.619 & 0.009 & 0.009 & 15 uniform \\
\hline M0765 & 256 & exp05 & 1024bit-ECFP6 & KNN & 0.002 & 0.755 & 0.679 & 0.029 & 0.027 & 9 distance \\
\hline M0766 & 256 & exp06 & 1024bit-ECFP6 & KNN & 0.008 & 0.716 & 0.637 & 0.020 & 0.012 & 13 uniform \\
\hline M0767 & 256 & exp07 & 1024bit-ECFP6 & KNN & 0.009 & 0.699 & 0.630 & 0.010 & 0.001 & 7 uniform \\
\hline M0768 & 256 & exp08 & 1024bit-ECFP6 & KNN & 0.012 & 0.969 & 0.786 & 0.008 & 0.004 & 3 distance \\
\hline M0769 & 256 & exp09 & 1024bit-ECFP6 & KNN & 0.013 & 0.950 & 0.757 & 0.026 & 0.013 & 1 uniform \\
\hline M0770 & 256 & $\exp 10$ & 1024bit-ECFP6 & KNN & 0.002 & 0.775 & 0.648 & 0.031 & 0.029 & 11 uniform \\
\hline M0771 & 256 & exp11 & 1024bit-ECFP6 & KNN & 0.015 & 0.762 & 0.645 & 0.012 & 0.003 & 15 uniform \\
\hline M0772 & 256 & $\exp 12$ & 1024bit-ECFP6 & KNN & 0.005 & 0.797 & 0.694 & 0.012 & 0.007 & 9 distance \\
\hline M0773 & 256 & exp13 & 1024bit-ECFP6 & KNN & 0.018 & 0.697 & 0.625 & 0.013 & 0.005 & 9 uniform \\
\hline M0774 & 256 & exp14 & 1024bit-ECFP6 & KNN & 0.051 & 0.681 & 0.608 & 0.032 & 0.019 & 3 uniform \\
\hline M0775 & 256 & $\exp 15$ & 1024bit-ECFP6 & KNN & 0.002 & 0.704 & 0.649 & 0.007 & 0.005 & 15 uniform \\
\hline M0776 & 256 & $\exp 16$ & 1024bit-ECFP6 & KNN & 0.008 & 0.745 & 0.651 & 0.014 & 0.006 & 5 distance \\
\hline M0777 & 256 & $\exp 17$ & 1024bit-ECFP6 & KNN & 0.004 & 0.953 & 0.737 & 0.027 & 0.023 & 3 distance \\
\hline M0778 & 256 & $\exp 18$ & 1024bit-ECFP6 & KNN & 0.001 & 0.774 & 0.669 & 0.009 & 0.008 & 15 distance \\
\hline M0779 & 256 & exp19 & 1024bit-ECFP6 & KNN & 0.032 & 0.886 & 0.697 & 0.013 & 0.019 & 5 uniform \\
\hline M0780 & 256 & exp01 & 1024bit-ECFP6 & SVM & 0.017 & 0.776 & 0.680 & 0.016 & 0.001 & $10000.01 \mathrm{e}-05$ \\
\hline M0781 & 256 & $\exp 02$ & 1024bit-ECFP6 & SVM & 0.000 & 0.871 & 0.693 & 0.015 & 0.015 & 50.05 \\
\hline M0782 & 256 & $\operatorname{exp03}$ & 1024bit-ECFP6 & SVM & 0.015 & 0.725 & 0.660 & 0.017 & 0.002 & 100.01 \\
\hline M0783 & 256 & exp04 & 1024bit-ECFP6 & SVM & 0.003 & 0.669 & 0.619 & 0.017 & 0.014 & $5001 \mathrm{e}-05$ \\
\hline M0784 & 256 & exp05 & 1024bit-ECFP6 & SVM & 0.027 & 0.937 & 0.731 & 0.024 & 0.003 & 10000.00 .0001 \\
\hline M0785 & 256 & exp06 & 1024bit-ECFP6 & SVM & 0.001 & 1.013 & 0.768 & 0.024 & 0.023 & 1000.005 \\
\hline M0786 & 256 & exp07 & 1024bit-ECFP6 & SVM & 0.000 & 0.704 & 0.629 & 0.025 & 0.025 & $5001 \mathrm{e}-05$ \\
\hline M0787 & 256 & $\exp 08$ & 1024bit-ECFP6 & SVM & 0.000 & 0.925 & 0.748 & 0.018 & 0.018 & $5000.05 \mathrm{e}-05$ \\
\hline M0788 & 256 & exp09 & 1024bit-ECFP6 & SVM & 0.014 & 1.462 & 0.940 & 0.025 & 0.011 & 100000.00 .0005 \\
\hline M0789 & 256 & $\exp 10$ & 1024bit-ECFP6 & SVM & 0.001 & 0.705 & 0.620 & 0.040 & 0.039 & $501 \mathrm{e}-05$ \\
\hline M0790 & 256 & exp11 & 1024bit-ECFP6 & SVM & 0.035 & 0.811 & 0.677 & 0.027 & 0.008 & $1000.01 \mathrm{e}-05$ \\
\hline M0791 & 256 & exp12 & 1024bit-ECFP6 & SVM & 0.008 & 0.737 & 0.650 & 0.024 & 0.016 & 500.001 \\
\hline M0792 & 256 & $\exp 13$ & 1024bit-ECFP6 & SVM & 0.038 & 0.682 & 0.612 & 0.012 & 0.026 & 10.0001 \\
\hline M0793 & 256 & $\exp 14$ & 1024bit-ECFP6 & SVM & 0.027 & 0.713 & 0.630 & 0.013 & 0.014 & 50.01 \\
\hline M0794 & 256 & $\exp 15$ & 1024bit-ECFP6 & SVM & 0.003 & 1.160 & 0.832 & 0.017 & 0.014 & 10000.00 .0001 \\
\hline M0795 & 256 & $\exp 16$ & 1024bit-ECFP6 & SVM & 0.015 & 0.717 & 0.642 & 0.019 & 0.004 & 50.1 \\
\hline M0796 & 256 & $\exp 17$ & 1024bit-ECFP6 & SVM & 0.001 & 0.999 & 0.789 & 0.040 & 0.039 & 5000.00 .01 \\
\hline M0797 & 256 & exp18 & 1024bit-ECFP6 & SVM & 0.172 & 0.667 & 0.611 & 0.010 & 0.162 & $11 \mathrm{e}-05$ \\
\hline M0798 & 256 & exp19 & 1024bit-ECFP6 & SVM & 0.068 & 0.807 & 0.669 & 0.015 & 0.054 & $5001 \mathrm{e}-05$ \\
\hline M0799 & 256 & exp01 & 1024bit-ECFP6 & GBM & 0.027 & 0.735 & 0.649 & 0.024 & 0.003 & 500.4 \\
\hline M0800 & 256 & $\exp 02$ & 1024bit-ECFP6 & GBM & 0.005 & 1.068 & 0.810 & 0.018 & 0.013 & 1000.9 \\
\hline M0801 & 256 & $\operatorname{exp03}$ & 1024bit-ECFP6 & GBM & 0.019 & 0.694 & 0.641 & 0.018 & 0.001 & 100.9 \\
\hline M0802 & 256 & exp04 & 1024bit-ECFP6 & GBM & 0.007 & 0.897 & 0.718 & 0.015 & 0.008 & 2000.6 \\
\hline
\end{tabular}


Supporting Information (Texts S1-S9, Figures S1-S41 and Tables S1-S12)

\begin{tabular}{|c|c|c|c|c|c|c|c|c|c|c|}
\hline M0803 & 256 & exp05 & 1024bit-ECFP6 & GBM & 0.011 & 0.873 & 0.733 & 0.028 & 0.017 & 1000.7 \\
\hline M0804 & 256 & exp06 & 1024bit-ECFP6 & GBM & 0.001 & 0.981 & 0.751 & 0.011 & 0.010 & 5000.1 \\
\hline M0805 & 256 & exp07 & 1024bit-ECFP6 & GBM & 0.005 & 0.725 & 0.634 & 0.021 & 0.016 & 100.6 \\
\hline M0806 & 256 & exp08 & 1024bit-ECFP6 & GBM & 0.001 & 0.703 & 0.636 & 0.013 & 0.013 & 100.3 \\
\hline M0807 & 256 & exp09 & 1024bit-ECFP6 & GBM & 0.019 & 0.925 & 0.761 & 0.028 & 0.009 & 10000.9 \\
\hline M0808 & 256 & exp10 & 1024bit-ECFP6 & GBM & 0.002 & 0.796 & 0.669 & 0.035 & 0.033 & 500.2 \\
\hline M0809 & 256 & exp11 & 1024bit-ECFP6 & GBM & 0.045 & 0.879 & 0.704 & 0.013 & 0.032 & 100.6 \\
\hline M0810 & 256 & exp12 & 1024bit-ECFP6 & GBM & 0.000 & 0.719 & 0.630 & 0.015 & 0.015 & 100.5 \\
\hline M0811 & 256 & exp13 & 1024bit-ECFP6 & GBM & 0.000 & 0.693 & 0.613 & 0.017 & 0.017 & 100.1 \\
\hline M0812 & 256 & exp14 & 1024bit-ECFP6 & GBM & 0.019 & 0.712 & 0.643 & 0.016 & 0.003 & 100.7 \\
\hline M0813 & 256 & exp15 & 1024bit-ECFP6 & GBM & 0.000 & 0.985 & 0.752 & 0.021 & 0.021 & 500.6 \\
\hline M0814 & 256 & exp16 & 1024bit-ECFP6 & GBM & 0.003 & 0.793 & 0.663 & 0.020 & 0.017 & 500.5 \\
\hline M0815 & 256 & exp17 & 1024bit-ECFP6 & GBM & 0.002 & 0.904 & 0.727 & 0.043 & 0.041 & 1000.3 \\
\hline M0816 & 256 & exp18 & 1024bit-ECFP6 & GBM & 0.007 & 0.667 & 0.609 & 0.015 & 0.008 & 100.2 \\
\hline M0817 & 256 & exp19 & 1024bit-ECFP6 & GBM & 0.025 & 1.356 & 0.890 & 0.024 & 0.001 & 1000.9 \\
\hline M0818 & 256 & exp01 & 1024bit-ECFP6 & $\mathrm{RF}$ & 0.028 & 0.712 & 0.641 & 0.009 & 0.019 & 300 \\
\hline M0819 & 256 & exp02 & 1024bit-ECFP6 & $\mathrm{RF}$ & 0.001 & 0.802 & 0.666 & 0.011 & 0.010 & 500 \\
\hline M0820 & 256 & exp03 & 1024bit-ECFP6 & $\mathrm{RF}$ & 0.049 & 0.657 & 0.631 & 0.005 & 0.044 & 50 \\
\hline M0821 & 256 & exp04 & 1024bit-ECFP6 & $\mathrm{RF}$ & 0.013 & 0.728 & 0.646 & 0.012 & 0.001 & 10 \\
\hline M0822 & 256 & exp05 & 1024bit-ECFP6 & $\mathrm{RF}$ & 0.005 & 0.754 & 0.682 & 0.023 & 0.018 & 50 \\
\hline M0823 & 256 & exp06 & 1024bit-ECFP6 & $\mathrm{RF}$ & 0.000 & 0.822 & 0.693 & 0.010 & 0.010 & 800 \\
\hline M0824 & 256 & exp07 & 1024bit-ECFP6 & $\mathrm{RF}$ & 0.004 & 0.780 & 0.671 & 0.007 & 0.003 & 50 \\
\hline M0825 & 256 & exp08 & 1024bit-ECFP6 & $\mathrm{RF}$ & 0.003 & 0.818 & 0.711 & 0.005 & 0.002 & 10 \\
\hline M0826 & 256 & exp09 & 1024bit-ECFP6 & $\mathrm{RF}$ & 0.043 & 0.678 & 0.625 & 0.019 & 0.024 & 400 \\
\hline M0827 & 256 & exp10 & 1024bit-ECFP6 & $\mathrm{RF}$ & 0.003 & 0.856 & 0.717 & 0.018 & 0.015 & 10 \\
\hline M0828 & 256 & exp11 & 1024bit-ECFP6 & $\mathrm{RF}$ & 0.038 & 0.937 & 0.738 & 0.010 & 0.028 & 50 \\
\hline M0829 & 256 & exp12 & 1024bit-ECFP6 & $\mathrm{RF}$ & 0.000 & 0.755 & 0.661 & 0.009 & 0.009 & 100 \\
\hline M0830 & 256 & exp13 & 1024bit-ECFP6 & $\mathrm{RF}$ & 0.002 & 0.808 & 0.676 & 0.009 & 0.007 & 10 \\
\hline M0831 & 256 & exp14 & 1024bit-ECFP6 & $\mathrm{RF}$ & 0.029 & 0.708 & 0.636 & 0.007 & 0.022 & 400 \\
\hline M0832 & 256 & exp15 & 1024bit-ECFP6 & $\mathrm{RF}$ & 0.001 & 0.826 & 0.699 & 0.009 & 0.008 & 400 \\
\hline M0833 & 256 & exp16 & 1024bit-ECFP6 & $\mathrm{RF}$ & 0.006 & 0.718 & 0.645 & 0.009 & 0.003 & 100 \\
\hline M0834 & 256 & exp17 & 1024bit-ECFP6 & $\mathrm{RF}$ & 0.001 & 0.851 & 0.708 & 0.029 & 0.028 & 50 \\
\hline M0835 & 256 & exp18 & 1024bit-ECFP6 & $\mathrm{RF}$ & 0.002 & 0.788 & 0.701 & 0.006 & 0.004 & 700 \\
\hline M0836 & 256 & exp19 & 1024bit-ECFP6 & $\mathrm{RF}$ & 0.011 & 0.976 & 0.744 & 0.011 & 0.000 & 10 \\
\hline M0837 & 256 & exp01 & 2048bit-ECFP6 & KNN & 0.006 & 0.781 & 0.685 & 0.020 & 0.014 & 9 distance \\
\hline M0838 & 256 & $\exp 02$ & 2048bit-ECFP6 & KNN & 0.000 & 0.741 & 0.647 & 0.017 & 0.017 & 15 uniform \\
\hline M0839 & 256 & exp03 & 2048bit-ECFP6 & KNN & 0.059 & 0.629 & 0.607 & 0.014 & 0.045 & 13 uniform \\
\hline M0840 & 256 & exp04 & 2048bit-ECFP6 & KNN & 0.004 & 0.780 & 0.663 & 0.010 & 0.006 & 5 distance \\
\hline M0841 & 256 & exp05 & 2048bit-ECFP6 & KNN & 0.004 & 0.735 & 0.664 & 0.026 & 0.022 & 13 distance \\
\hline M0842 & 256 & exp06 & 2048bit-ECFP6 & KNN & 0.000 & 0.778 & 0.663 & 0.035 & 0.035 & 9 uniform \\
\hline M0843 & 256 & exp07 & 2048bit-ECFP6 & KNN & 0.006 & 1.010 & 0.790 & 0.018 & 0.012 & 1 uniform \\
\hline M0844 & 256 & exp08 & 2048bit-ECFP6 & KNN & 0.007 & 0.940 & 0.786 & 0.008 & 0.001 & 3 distance \\
\hline M0845 & 256 & exp09 & 2048bit-ECFP6 & KNN & 0.002 & 1.008 & 0.767 & 0.018 & 0.016 & 1 uniform \\
\hline M0846 & 256 & exp10 & 2048bit-ECFP6 & KNN & 0.000 & 0.746 & 0.639 & 0.029 & 0.029 & 15 uniform \\
\hline M0847 & 256 & exp11 & 2048bit-ECFP6 & KNN & 0.044 & 1.378 & 0.854 & 0.010 & 0.034 & 1 distance \\
\hline
\end{tabular}


Supporting Information (Texts S1-S9, Figures S1-S41 and Tables S1-S12)

\begin{tabular}{|c|c|c|c|c|c|c|c|c|c|c|}
\hline M0848 & 256 & exp12 & 2048bit-ECFP6 & KNN & 0.007 & 0.788 & 0.692 & 0.010 & 0.003 & 13 distance \\
\hline M0849 & 256 & exp13 & 2048bit-ECFP6 & KNN & 0.001 & 0.768 & 0.662 & 0.011 & 0.010 & 5 uniform \\
\hline M0850 & 256 & exp14 & 2048bit-ECFP6 & KNN & 0.060 & 0.659 & 0.604 & 0.021 & 0.038 & 5 uniform \\
\hline M0851 & 256 & exp15 & 2048bit-ECFP6 & KNN & 0.000 & 0.771 & 0.675 & 0.003 & 0.003 & 5 uniform \\
\hline M0852 & 256 & exp16 & 2048bit-ECFP6 & KNN & 0.005 & 0.660 & 0.602 & 0.019 & 0.014 & 11 uniform \\
\hline M0853 & 256 & exp17 & 2048bit-ECFP6 & KNN & 0.009 & 1.140 & 0.801 & 0.028 & 0.019 & 1 uniform \\
\hline M0854 & 256 & exp18 & 2048bit-ECFP6 & KNN & 0.000 & 0.875 & 0.735 & 0.008 & 0.008 & 5 distance \\
\hline M0855 & 256 & exp19 & 2048bit-ECFP6 & KNN & 0.026 & 0.795 & 0.669 & 0.012 & 0.014 & 9 uniform \\
\hline M0856 & 256 & exp01 & 2048bit-ECFP6 & SVM & 0.005 & 0.869 & 0.728 & 0.025 & 0.020 & $5000.05 \mathrm{e}-05$ \\
\hline M0857 & 256 & exp02 & 2048bit-ECFP6 & SVM & 0.002 & 1.199 & 0.811 & 0.022 & 0.020 & 100000.00 .0001 \\
\hline M0858 & 256 & exp03 & 2048bit-ECFP6 & SVM & 0.002 & 0.676 & 0.626 & 0.021 & 0.019 & $505 \mathrm{e}-05$ \\
\hline M0859 & 256 & exp04 & 2048bit-ECFP6 & SVM & 0.001 & 0.660 & 0.608 & 0.013 & 0.012 & $1001 \mathrm{e}-05$ \\
\hline M0860 & 256 & exp05 & 2048bit-ECFP6 & SVM & 0.011 & 1.100 & 0.827 & 0.019 & 0.009 & $50000.05 \mathrm{e}-05$ \\
\hline M0861 & 256 & exp06 & 2048bit-ECFP6 & SVM & 0.007 & 0.701 & 0.617 & 0.024 & 0.017 & $11 \mathrm{e}-05$ \\
\hline M0862 & 256 & exp07 & 2048bit-ECFP6 & SVM & 0.001 & 0.723 & 0.643 & 0.041 & 0.040 & $1000.01 \mathrm{e}-05$ \\
\hline M0863 & 256 & exp08 & 2048bit-ECFP6 & SVM & 0.001 & 1.111 & 0.828 & 0.010 & 0.009 & 50000.00 .0001 \\
\hline M0864 & 256 & exp09 & 2048bit-ECFP6 & SVM & 0.001 & 0.923 & 0.735 & 0.017 & 0.016 & 100000.00 .05 \\
\hline M0865 & 256 & $\exp 10$ & 2048bit-ECFP6 & SVM & 0.000 & 0.754 & 0.649 & 0.043 & 0.043 & $1000.01 \mathrm{e}-05$ \\
\hline M0866 & 256 & exp11 & 2048bit-ECFP6 & SVM & 0.028 & 0.936 & 0.743 & 0.022 & 0.006 & 1000.00 .0001 \\
\hline M0867 & 256 & exp12 & 2048bit-ECFP6 & SVM & 0.004 & 0.767 & 0.671 & 0.027 & 0.023 & 10.05 \\
\hline M0868 & 256 & exp13 & 2048bit-ECFP6 & SVM & 0.053 & 0.682 & 0.613 & 0.014 & 0.039 & 10.0001 \\
\hline M0869 & 256 & exp14 & 2048bit-ECFP6 & SVM & 0.001 & 1.184 & 0.844 & 0.018 & 0.017 & 50000.00 .001 \\
\hline M0870 & 256 & exp15 & 2048bit-ECFP6 & SVM & 0.000 & 0.882 & 0.713 & 0.039 & 0.039 & $10000.01 \mathrm{e}-05$ \\
\hline M0871 & 256 & exp16 & 2048bit-ECFP6 & SVM & 0.000 & 0.789 & 0.673 & 0.017 & 0.017 & 100.01 \\
\hline M0872 & 256 & exp17 & 2048bit-ECFP6 & SVM & 0.000 & 0.926 & 0.750 & 0.047 & 0.047 & 500.05 \\
\hline M0873 & 256 & exp18 & 2048bit-ECFP6 & SVM & 0.007 & 1.187 & 0.884 & 0.014 & 0.007 & 10000.00 .001 \\
\hline M0874 & 256 & exp19 & 2048bit-ECFP6 & SVM & 0.046 & 0.818 & 0.674 & 0.019 & 0.027 & $1000.01 \mathrm{e}-05$ \\
\hline M0875 & 256 & exp01 & 2048bit-ECFP6 & GBM & 0.002 & 0.927 & 0.761 & 0.017 & 0.015 & 6000.2 \\
\hline M0876 & 256 & exp02 & 2048bit-ECFP6 & GBM & 0.000 & 0.940 & 0.738 & 0.035 & 0.035 & 1000.7 \\
\hline M0877 & 256 & exp03 & 2048bit-ECFP6 & GBM & 0.006 & 0.694 & 0.636 & 0.014 & 0.008 & 100.6 \\
\hline M0878 & 256 & exp04 & 2048bit-ECFP6 & GBM & 0.017 & 0.909 & 0.752 & 0.021 & 0.004 & 10000.9 \\
\hline M0879 & 256 & exp05 & 2048bit-ECFP6 & GBM & 0.003 & 0.826 & 0.704 & 0.030 & 0.027 & 2000.1 \\
\hline M0880 & 256 & exp06 & 2048bit-ECFP6 & GBM & 0.010 & 1.138 & 0.764 & 0.013 & 0.003 & 100.9 \\
\hline M0881 & 256 & exp07 & 2048bit-ECFP6 & GBM & 0.001 & 0.686 & 0.614 & 0.022 & 0.021 & 100.1 \\
\hline M0882 & 256 & exp08 & 2048bit-ECFP6 & GBM & 0.002 & 0.669 & 0.613 & 0.021 & 0.019 & 100.1 \\
\hline M0883 & 256 & exp09 & 2048bit-ECFP6 & GBM & 0.009 & 1.008 & 0.754 & 0.021 & 0.012 & 3000.9 \\
\hline M0884 & 256 & exp10 & 2048bit-ECFP6 & GBM & 0.005 & 0.867 & 0.710 & 0.043 & 0.038 & 2000.1 \\
\hline M0885 & 256 & exp11 & 2048bit-ECFP6 & GBM & 0.037 & 1.063 & 0.779 & 0.019 & 0.018 & 500.8 \\
\hline M0886 & 256 & exp12 & 2048bit-ECFP6 & GBM & 0.000 & 0.663 & 0.607 & 0.018 & 0.018 & 100.1 \\
\hline M0887 & 256 & exp13 & 2048bit-ECFP6 & GBM & 0.004 & 0.854 & 0.696 & 0.011 & 0.007 & 500.3 \\
\hline M0888 & 256 & exp14 & 2048bit-ECFP6 & GBM & 0.019 & 0.868 & 0.717 & 0.012 & 0.007 & 1000.8 \\
\hline M0889 & 256 & exp15 & 2048bit-ECFP6 & GBM & 0.000 & 1.068 & 0.798 & 0.026 & 0.026 & 9000.6 \\
\hline M0890 & 256 & exp16 & 2048bit-ECFP6 & GBM & 0.010 & 0.655 & 0.618 & 0.024 & 0.014 & 100.3 \\
\hline M0891 & 256 & exp17 & 2048bit-ECFP6 & GBM & 0.002 & 0.750 & 0.639 & 0.037 & 0.035 & 100.4 \\
\hline M0892 & 256 & $\exp 18$ & 2048bit-ECFP6 & GBM & 0.005 & 0.665 & 0.608 & 0.046 & 0.041 & 100.1 \\
\hline
\end{tabular}


Supporting Information (Texts S1-S9, Figures S1-S41 and Tables S1-S12)

\begin{tabular}{|c|c|c|c|c|c|c|c|c|c|c|}
\hline M0893 & 256 & exp19 & 2048bit-ECFP6 & GBM & 0.010 & 1.346 & 0.903 & 0.009 & 0.001 & 9000.7 \\
\hline M0894 & 256 & exp01 & 2048bit-ECFP6 & $\mathrm{RF}$ & 0.011 & 0.766 & 0.691 & 0.014 & 0.003 & 10 \\
\hline M0895 & 256 & exp02 & 2048bit-ECFP6 & $\mathrm{RF}$ & 0.004 & 0.787 & 0.659 & 0.013 & 0.009 & 400 \\
\hline M0896 & 256 & exp03 & 2048bit-ECFP6 & $\mathrm{RF}$ & 0.027 & 0.701 & 0.639 & 0.003 & 0.024 & 10 \\
\hline M0897 & 256 & exp04 & 2048bit-ECFP6 & $\mathrm{RF}$ & 0.010 & 0.720 & 0.657 & 0.010 & 0.000 & 50 \\
\hline M0898 & 256 & exp05 & 2048bit-ECFP6 & $\mathrm{RF}$ & 0.003 & 0.798 & 0.708 & 0.023 & 0.020 & 1000 \\
\hline M0899 & 256 & exp06 & 2048bit-ECFP6 & $\mathrm{RF}$ & 0.005 & 0.991 & 0.755 & 0.013 & 0.008 & 900 \\
\hline M0900 & 256 & exp07 & 2048bit-ECFP6 & $\mathrm{RF}$ & 0.002 & 0.807 & 0.684 & 0.009 & 0.007 & 900 \\
\hline M0901 & 256 & exp08 & 2048bit-ECFP6 & $\mathrm{RF}$ & 0.000 & 0.822 & 0.723 & 0.005 & 0.005 & 800 \\
\hline M0902 & 256 & exp09 & 2048bit-ECFP6 & $\mathrm{RF}$ & 0.025 & 0.715 & 0.644 & 0.016 & 0.009 & 200 \\
\hline M0903 & 256 & exp10 & 2048bit-ECFP6 & $\mathrm{RF}$ & 0.004 & 0.856 & 0.713 & 0.015 & 0.011 & 50 \\
\hline M0904 & 256 & $\exp 11$ & 2048bit-ECFP6 & $\mathrm{RF}$ & 0.018 & 0.914 & 0.733 & 0.011 & 0.007 & 50 \\
\hline M0905 & 256 & exp12 & 2048bit-ECFP6 & $\mathrm{RF}$ & 0.003 & 0.810 & 0.690 & 0.007 & 0.004 & 100 \\
\hline M0906 & 256 & exp13 & 2048bit-ECFP6 & $\mathrm{RF}$ & 0.001 & 0.849 & 0.712 & 0.005 & 0.004 & 400 \\
\hline M0907 & 256 & exp14 & 2048bit-ECFP6 & $\mathrm{RF}$ & 0.030 & 0.710 & 0.633 & 0.009 & 0.021 & 50 \\
\hline M0908 & 256 & exp15 & 2048bit-ECFP6 & $\mathrm{RF}$ & 0.000 & 0.848 & 0.711 & 0.010 & 0.010 & 1000 \\
\hline M0909 & 256 & exp16 & 2048bit-ECFP6 & $\mathrm{RF}$ & 0.016 & 0.720 & 0.653 & 0.015 & 0.001 & 10 \\
\hline M0910 & 256 & exp17 & 2048bit-ECFP6 & $\mathrm{RF}$ & 0.001 & 0.862 & 0.706 & 0.038 & 0.037 & 50 \\
\hline M0911 & 256 & exp18 & 2048bit-ECFP6 & $\mathrm{RF}$ & 0.001 & 0.799 & 0.711 & 0.006 & 0.005 & 600 \\
\hline M0912 & 256 & exp19 & 2048bit-ECFP6 & $\mathrm{RF}$ & 0.014 & 1.006 & 0.773 & 0.009 & 0.005 & 10 \\
\hline M0913 & 128 & exp01 & 1024bit-ECFP4 & KNN & 0.023 & 0.696 & 0.623 & 0.029 & 0.006 & 7 uniform \\
\hline M0914 & 128 & exp02 & 1024bit-ECFP4 & KNN & 0.001 & 0.895 & 0.712 & 0.014 & 0.013 & 7 distance \\
\hline M0915 & 128 & exp03 & 1024bit-ECFP4 & KNN & 0.029 & 0.835 & 0.707 & 0.012 & 0.017 & 1 uniform \\
\hline M0916 & 128 & exp04 & 1024bit-ECFP4 & KNN & 0.003 & 0.692 & 0.626 & 0.012 & 0.009 & 7 uniform \\
\hline M0917 & 128 & exp05 & 1024bit-ECFP4 & KNN & 0.001 & 0.804 & 0.702 & 0.027 & 0.026 & 9 distance \\
\hline M0918 & 128 & exp06 & 1024bit-ECFP4 & KNN & 0.001 & 0.749 & 0.643 & 0.020 & 0.019 & 11 uniform \\
\hline M0919 & 128 & exp07 & 1024bit-ECFP4 & KNN & 0.004 & 0.784 & 0.669 & 0.018 & 0.014 & 3 uniform \\
\hline M0920 & 128 & exp08 & 1024bit-ECFP4 & KNN & 0.000 & 1.028 & 0.803 & 0.008 & 0.008 & 1 uniform \\
\hline M0921 & 128 & exp09 & 1024bit-ECFP4 & KNN & 0.001 & 0.739 & 0.642 & 0.020 & 0.019 & 5 uniform \\
\hline M0922 & 128 & $\exp 10$ & 1024bit-ECFP4 & KNN & 0.001 & 0.789 & 0.670 & 0.024 & 0.023 & 9 uniform \\
\hline M0923 & 128 & exp11 & 1024bit-ECFP4 & KNN & 0.009 & 0.752 & 0.646 & 0.006 & 0.003 & 15 uniform \\
\hline M0924 & 128 & exp12 & 1024bit-ECFP4 & KNN & 0.002 & 1.072 & 0.779 & 0.013 & 0.011 & 1 uniform \\
\hline M0925 & 128 & exp13 & 1024bit-ECFP4 & KNN & 0.000 & 0.718 & 0.617 & 0.013 & 0.013 & 15 uniform \\
\hline M0926 & 128 & exp14 & 1024bit-ECFP4 & KNN & 0.013 & 0.949 & 0.745 & 0.020 & 0.007 & 1 uniform \\
\hline M0927 & 128 & exp15 & 1024bit-ECFP4 & KNN & 0.014 & 0.757 & 0.663 & 0.009 & 0.005 & 13 uniform \\
\hline M0928 & 128 & exp16 & 1024bit-ECFP4 & KNN & 0.000 & 0.738 & 0.643 & 0.018 & 0.018 & 5 uniform \\
\hline M0929 & 128 & exp17 & 1024bit-ECFP4 & KNN & 0.006 & 1.012 & 0.774 & 0.037 & 0.031 & 3 distance \\
\hline M0930 & 128 & exp18 & 1024bit-ECFP4 & KNN & 0.012 & 0.750 & 0.679 & 0.006 & 0.006 & 3 uniform \\
\hline M0931 & 128 & exp19 & 1024bit-ECFP4 & KNN & 0.010 & 1.396 & 0.892 & 0.017 & 0.007 & 1 uniform \\
\hline M0932 & 128 & exp01 & 1024bit-ECFP4 & SVM & 0.030 & 0.749 & 0.660 & 0.012 & 0.018 & 100.1 \\
\hline M0933 & 128 & exp02 & 1024bit-ECFP4 & SVM & 0.002 & 0.734 & 0.633 & 0.018 & 0.016 & $505 \mathrm{e}-05$ \\
\hline M0934 & 128 & exp03 & 1024bit-ECFP4 & SVM & 0.020 & 0.773 & 0.677 & 0.029 & 0.009 & 10000.00 .0005 \\
\hline M0935 & 128 & exp04 & 1024bit-ECFP4 & SVM & 0.002 & 0.804 & 0.696 & 0.009 & 0.007 & 50.1 \\
\hline M0936 & 128 & exp05 & 1024bit-ECFP4 & SVM & 0.006 & 0.875 & 0.743 & 0.018 & 0.012 & 50000.00 .1 \\
\hline M0937 & 128 & $\exp 06$ & 1024bit-ECFP4 & SVM & 0.003 & 0.807 & 0.678 & 0.030 & 0.028 & $5000.01 \mathrm{e}-05$ \\
\hline
\end{tabular}


Supporting Information (Texts S1-S9, Figures S1-S41 and Tables S1-S12)

\begin{tabular}{|c|c|c|c|c|c|c|c|c|c|c|}
\hline M0938 & 128 & exp07 & 1024bit-ECFP4 & SVM & 0.000 & 0.756 & 0.675 & 0.044 & 0.044 & $5000.01 \mathrm{e}-05$ \\
\hline M0939 & 128 & exp08 & 1024bit-ECFP4 & SVM & 0.004 & 0.690 & 0.626 & 0.010 & 0.006 & $1000.01 \mathrm{e}-05$ \\
\hline M0940 & 128 & exp09 & 1024bit-ECFP4 & SVM & 0.015 & 0.972 & 0.729 & 0.032 & 0.017 & 100000.00 .001 \\
\hline M0941 & 128 & exp10 & 1024bit-ECFP4 & SVM & 0.007 & 1.003 & 0.757 & 0.080 & 0.073 & $100000.01 \mathrm{e}-05$ \\
\hline M0942 & 128 & exp11 & 1024bit-ECFP4 & SVM & 0.013 & 1.335 & 0.896 & 0.012 & 0.001 & 100000.00 .005 \\
\hline M0943 & 128 & exp12 & 1024bit-ECFP4 & SVM & 0.000 & 0.827 & 0.698 & 0.025 & 0.025 & 50000.00 .0001 \\
\hline M0944 & 128 & exp13 & 1024bit-ECFP4 & SVM & 0.074 & 0.679 & 0.612 & 0.011 & 0.063 & $55 \mathrm{e}-05$ \\
\hline M0945 & 128 & exp14 & 1024bit-ECFP4 & SVM & 0.049 & 0.669 & 0.597 & 0.014 & 0.035 & 10.1 \\
\hline M0946 & 128 & exp15 & 1024bit-ECFP4 & SVM & 0.000 & 0.904 & 0.739 & 0.026 & 0.026 & $100000.01 \mathrm{e}-05$ \\
\hline M0947 & 128 & exp16 & 1024bit-ECFP4 & SVM & 0.000 & 0.800 & 0.691 & 0.018 & 0.018 & 50.05 \\
\hline M0948 & 128 & exp17 & 1024bit-ECFP4 & SVM & 0.000 & 1.028 & 0.802 & 0.039 & 0.039 & 500.05 \\
\hline M0949 & 128 & exp18 & 1024bit-ECFP4 & SVM & 0.109 & 0.668 & 0.612 & 0.011 & 0.099 & $11 \mathrm{e}-05$ \\
\hline M0950 & 128 & exp19 & 1024bit-ECFP4 & SVM & 0.016 & 1.044 & 0.762 & 0.016 & 0.000 & $50000.01 \mathrm{e}-05$ \\
\hline M0951 & 128 & exp01 & 1024bit-ECFP4 & GBM & 0.027 & 0.702 & 0.621 & 0.016 & 0.011 & 100.6 \\
\hline M0952 & 128 & exp02 & 1024bit-ECFP4 & GBM & 0.008 & 0.989 & 0.740 & 0.018 & 0.010 & 500.8 \\
\hline M0953 & 128 & exp03 & 1024bit-ECFP4 & GBM & 0.022 & 0.658 & 0.609 & 0.013 & 0.009 & 100.1 \\
\hline M0954 & 128 & exp04 & 1024bit-ECFP4 & GBM & 0.002 & 0.957 & 0.762 & 0.016 & 0.014 & 3000.6 \\
\hline M0955 & 128 & exp05 & 1024bit-ECFP4 & GBM & 0.003 & 1.003 & 0.777 & 0.023 & 0.020 & 5000.4 \\
\hline M0956 & 128 & exp06 & 1024bit-ECFP4 & GBM & 0.001 & 0.866 & 0.706 & 0.019 & 0.018 & 1000.2 \\
\hline M0957 & 128 & exp07 & 1024bit-ECFP4 & GBM & 0.005 & 0.719 & 0.642 & 0.022 & 0.017 & 100.6 \\
\hline M0958 & 128 & exp08 & 1024bit-ECFP4 & GBM & 0.007 & 0.723 & 0.646 & 0.021 & 0.014 & 500.1 \\
\hline M0959 & 128 & exp09 & 1024bit-ECFP4 & GBM & 0.028 & 0.781 & 0.678 & 0.030 & 0.002 & 7000.2 \\
\hline M0960 & 128 & exp10 & 1024bit-ECFP4 & GBM & 0.000 & 0.849 & 0.695 & 0.070 & 0.070 & 2000.1 \\
\hline M0961 & 128 & $\operatorname{exp11}$ & 1024bit-ECFP4 & GBM & 0.007 & 1.137 & 0.818 & 0.010 & 0.003 & 7000.8 \\
\hline M0962 & 128 & exp12 & 1024bit-ECFP4 & GBM & 0.025 & 0.889 & 0.723 & 0.013 & 0.012 & 500.5 \\
\hline M0963 & 128 & exp13 & 1024bit-ECFP4 & GBM & 0.008 & 0.689 & 0.620 & 0.013 & 0.005 & 100.3 \\
\hline M0964 & 128 & exp14 & 1024bit-ECFP4 & GBM & 0.037 & 0.681 & 0.606 & 0.011 & 0.026 & 100.5 \\
\hline M0965 & 128 & exp15 & 1024bit-ECFP4 & GBM & 0.015 & 0.885 & 0.720 & 0.025 & 0.010 & 100.8 \\
\hline M0966 & 128 & exp16 & 1024bit-ECFP4 & GBM & 0.001 & 1.043 & 0.779 & 0.023 & 0.022 & 10000.9 \\
\hline M0967 & 128 & exp17 & 1024bit-ECFP4 & GBM & 0.002 & 0.949 & 0.733 & 0.031 & 0.029 & 1000.3 \\
\hline M0968 & 128 & exp18 & 1024bit-ECFP4 & GBM & 0.005 & 0.737 & 0.666 & 0.018 & 0.013 & 100.9 \\
\hline M0969 & 128 & exp19 & 1024bit-ECFP4 & GBM & 0.040 & 1.529 & 0.917 & 0.023 & 0.017 & 3000.8 \\
\hline M0970 & 128 & exp01 & 1024bit-ECFP4 & $\mathrm{RF}$ & 0.037 & 0.720 & 0.639 & 0.013 & 0.024 & 900 \\
\hline M0971 & 128 & exp02 & 1024bit-ECFP4 & $\mathrm{RF}$ & 0.004 & 0.871 & 0.703 & 0.007 & 0.003 & 500 \\
\hline M0972 & 128 & $\operatorname{exp03}$ & 1024bit-ECFP4 & $\mathrm{RF}$ & 0.039 & 0.670 & 0.644 & 0.004 & 0.035 & 500 \\
\hline M0973 & 128 & exp04 & 1024bit-ECFP4 & $\mathrm{RF}$ & 0.001 & 0.782 & 0.683 & 0.008 & 0.007 & 200 \\
\hline M0974 & 128 & exp05 & 1024bit-ECFP4 & $\mathrm{RF}$ & 0.009 & 0.759 & 0.689 & 0.014 & 0.005 & 300 \\
\hline M0975 & 128 & exp06 & 1024bit-ECFP4 & $\mathrm{RF}$ & 0.001 & 0.886 & 0.724 & 0.009 & 0.008 & 50 \\
\hline M0976 & 128 & exp07 & 1024bit-ECFP4 & $\mathrm{RF}$ & 0.001 & 0.869 & 0.720 & 0.018 & 0.017 & 10 \\
\hline M0977 & 128 & exp08 & 1024bit-ECFP4 & $\mathrm{RF}$ & 0.000 & 0.822 & 0.708 & 0.007 & 0.007 & 10 \\
\hline M0978 & 128 & exp09 & 1024bit-ECFP4 & $\mathrm{RF}$ & 0.036 & 0.682 & 0.621 & 0.029 & 0.007 & 100 \\
\hline M0979 & 128 & exp10 & 1024bit-ECFP4 & $\mathrm{RF}$ & 0.007 & 0.897 & 0.722 & 0.041 & 0.034 & 100 \\
\hline M0980 & 128 & exp11 & 1024bit-ECFP4 & $\mathrm{RF}$ & 0.008 & 0.958 & 0.742 & 0.007 & 0.001 & 10 \\
\hline M0981 & 128 & exp12 & 1024bit-ECFP4 & $\mathrm{RF}$ & 0.015 & 0.856 & 0.708 & 0.007 & 0.008 & 900 \\
\hline M0982 & 128 & $\exp 13$ & 1024bit-ECFP4 & $\mathrm{RF}$ & 0.002 & 0.794 & 0.668 & 0.004 & 0.002 & 1000 \\
\hline
\end{tabular}


Supporting Information (Texts S1-S9, Figures S1-S41 and Tables S1-S12)

\begin{tabular}{|c|c|c|c|c|c|c|c|c|c|c|}
\hline M0983 & 128 & $\exp 14$ & 1024bit-ECFP4 & $\mathrm{RF}$ & 0.024 & 0.737 & 0.642 & 0.005 & 0.019 & 200 \\
\hline M0984 & 128 & exp15 & 1024bit-ECFP4 & $\mathrm{RF}$ & 0.010 & 0.915 & 0.744 & 0.012 & 0.002 & 400 \\
\hline M0985 & 128 & exp16 & 1024bit-ECFP4 & $\mathrm{RF}$ & 0.000 & 0.775 & 0.664 & 0.012 & 0.012 & 500 \\
\hline M0986 & 128 & exp17 & 1024bit-ECFP4 & $\mathrm{RF}$ & 0.001 & 0.891 & 0.726 & 0.029 & 0.028 & 400 \\
\hline M0987 & 128 & exp18 & 1024bit-ECFP4 & $\mathrm{RF}$ & 0.007 & 0.767 & 0.690 & 0.005 & 0.002 & 10 \\
\hline M0988 & 128 & exp19 & 1024bit-ECFP4 & $\mathrm{RF}$ & 0.026 & 1.055 & 0.765 & 0.012 & 0.014 & 10 \\
\hline M0989 & 128 & exp01 & 2048bit-ECFP4 & KNN & 0.009 & 0.693 & 0.622 & 0.022 & 0.013 & 15 uniform \\
\hline M0990 & 128 & exp02 & 2048bit-ECFP4 & KNN & 0.006 & 0.781 & 0.655 & 0.010 & 0.004 & 3 uniform \\
\hline M0991 & 128 & exp03 & 2048bit-ECFP4 & KNN & 0.013 & 0.667 & 0.611 & 0.013 & 0.000 & 15 uniform \\
\hline M0992 & 128 & exp04 & 2048bit-ECFP4 & KNN & 0.006 & 0.669 & 0.621 & 0.013 & 0.007 & 11 uniform \\
\hline M0993 & 128 & exp05 & 2048bit-ECFP4 & KNN & 0.000 & 0.890 & 0.753 & 0.028 & 0.028 & 3 distance \\
\hline M0994 & 128 & exp06 & 2048bit-ECFP4 & KNN & 0.001 & 0.978 & 0.764 & 0.025 & 0.024 & 5 distance \\
\hline M0995 & 128 & exp07 & 2048bit-ECFP4 & KNN & 0.001 & 1.053 & 0.792 & 0.014 & 0.013 & 1 distance \\
\hline M0996 & 128 & exp08 & 2048bit-ECFP4 & KNN & 0.006 & 0.925 & 0.762 & 0.007 & 0.001 & 5 distance \\
\hline M0997 & 128 & exp09 & 2048bit-ECFP4 & KNN & 0.010 & 0.765 & 0.676 & 0.019 & 0.009 & 3 uniform \\
\hline M0998 & 128 & exp10 & 2048bit-ECFP4 & KNN & 0.009 & 0.793 & 0.665 & 0.021 & 0.012 & 9 uniform \\
\hline M0999 & 128 & exp11 & 2048bit-ECFP4 & KNN & 0.054 & 0.787 & 0.659 & 0.010 & 0.044 & 13 uniform \\
\hline M1000 & 128 & $\exp 12$ & 2048bit-ECFP4 & KNN & 0.000 & 0.759 & 0.658 & 0.012 & 0.012 & 5 uniform \\
\hline M1001 & 128 & exp13 & 2048bit-ECFP4 & KNN & 0.011 & 0.785 & 0.663 & 0.011 & 0.000 & 3 uniform \\
\hline M1002 & 128 & exp14 & 2048bit-ECFP4 & KNN & 0.118 & 0.633 & 0.588 & 0.032 & 0.086 & 9 uniform \\
\hline M1003 & 128 & $\exp 15$ & 2048bit-ECFP4 & KNN & 0.000 & 0.845 & 0.713 & 0.005 & 0.005 & 3 uniform \\
\hline M1004 & 128 & exp16 & 2048bit-ECFP4 & KNN & 0.001 & 0.719 & 0.651 & 0.015 & 0.014 & 7 uniform \\
\hline M1005 & 128 & exp17 & 2048bit-ECFP4 & KNN & 0.000 & 0.985 & 0.756 & 0.047 & 0.047 & 3 distance \\
\hline M1006 & 128 & exp18 & 2048bit-ECFP4 & KNN & 0.001 & 0.707 & 0.636 & 0.015 & 0.014 & 15 uniform \\
\hline M1007 & 128 & $\exp 19$ & 2048bit-ECFP4 & KNN & 0.035 & 0.813 & 0.684 & 0.015 & 0.020 & 7 uniform \\
\hline M1008 & 128 & exp01 & 2048bit-ECFP4 & SVM & 0.020 & 0.872 & 0.709 & 0.021 & 0.001 & 100000.00 .0005 \\
\hline M1009 & 128 & exp02 & 2048bit-ECFP4 & SVM & 0.000 & 0.716 & 0.621 & 0.015 & 0.015 & $501 \mathrm{e}-05$ \\
\hline M1010 & 128 & exp03 & 2048bit-ECFP4 & SVM & 0.013 & 0.715 & 0.645 & 0.027 & 0.014 & 500.005 \\
\hline M1011 & 128 & exp04 & 2048bit-ECFP4 & SVM & 0.018 & 0.726 & 0.665 & 0.028 & 0.010 & $50000.01 \mathrm{e}-05$ \\
\hline M1012 & 128 & $\exp 05$ & 2048bit-ECFP4 & SVM & 0.006 & 0.800 & 0.700 & 0.021 & 0.015 & $50000.01 \mathrm{e}-05$ \\
\hline M1013 & 128 & exp06 & 2048bit-ECFP4 & SVM & 0.024 & 0.853 & 0.698 & 0.038 & 0.014 & $5000.01 \mathrm{e}-05$ \\
\hline M1014 & 128 & exp07 & 2048bit-ECFP4 & SVM & 0.001 & 0.796 & 0.701 & 0.022 & 0.021 & $50000.01 \mathrm{e}-05$ \\
\hline M1015 & 128 & exp08 & 2048bit-ECFP4 & SVM & 0.001 & 0.733 & 0.656 & 0.013 & 0.012 & $5000.05 \mathrm{e}-05$ \\
\hline M1016 & 128 & exp09 & 2048bit-ECFP4 & SVM & 0.013 & 0.814 & 0.689 & 0.023 & 0.010 & 100.1 \\
\hline M1017 & 128 & $\exp 10$ & 2048bit-ECFP4 & SVM & 0.001 & 0.774 & 0.661 & 0.055 & 0.054 & $5005 \mathrm{e}-05$ \\
\hline M1018 & 128 & $\exp 11$ & 2048bit-ECFP4 & SVM & 0.012 & 0.758 & 0.651 & 0.018 & 0.006 & $5005 \mathrm{e}-05$ \\
\hline M1019 & 128 & $\exp 12$ & 2048bit-ECFP4 & SVM & 0.001 & 0.763 & 0.680 & 0.023 & 0.022 & 5000.0005 \\
\hline M1020 & 128 & exp13 & 2048bit-ECFP4 & SVM & 0.054 & 0.675 & 0.612 & 0.011 & 0.043 & $501 \mathrm{e}-05$ \\
\hline M1021 & 128 & exp14 & 2048bit-ECFP4 & SVM & 0.051 & 0.665 & 0.601 & 0.020 & 0.031 & 10.05 \\
\hline M1022 & 128 & exp15 & 2048bit-ECFP4 & SVM & 0.010 & 1.044 & 0.796 & 0.025 & 0.015 & 50000.00 .0001 \\
\hline M1023 & 128 & exp16 & 2048bit-ECFP4 & SVM & 0.000 & 0.841 & 0.685 & 0.018 & 0.018 & $50000.05 \mathrm{e}-05$ \\
\hline M1024 & 128 & $\exp 17$ & 2048bit-ECFP4 & SVM & 0.001 & 0.972 & 0.765 & 0.049 & 0.048 & 500.05 \\
\hline M1025 & 128 & $\exp 18$ & 2048bit-ECFP4 & SVM & 0.008 & 0.791 & 0.711 & 0.016 & 0.008 & 1000.00 .001 \\
\hline M1026 & 128 & $\exp 19$ & 2048bit-ECFP4 & SVM & 0.023 & 1.129 & 0.801 & 0.017 & 0.006 & 500.1 \\
\hline M1027 & 128 & exp01 & 2048bit-ECFP4 & GBM & 0.013 & 0.685 & 0.611 & 0.013 & 0.000 & 100.1 \\
\hline
\end{tabular}


Supporting Information (Texts S1-S9, Figures S1-S41 and Tables S1-S12)

\begin{tabular}{|c|c|c|c|c|c|c|c|c|c|c|}
\hline M1028 & 128 & exp02 & 2048bit-ECFP4 & GBM & 0.002 & 0.742 & 0.643 & 0.009 & 0.007 & 100.3 \\
\hline M1029 & 128 & exp03 & 2048bit-ECFP4 & GBM & 0.048 & 0.649 & 0.602 & 0.015 & 0.033 & 100.1 \\
\hline M1030 & 128 & exp04 & 2048bit-ECFP4 & GBM & 0.015 & 0.687 & 0.636 & 0.021 & 0.006 & 100.9 \\
\hline M1031 & 128 & exp05 & 2048bit-ECFP4 & GBM & 0.003 & 1.148 & 0.816 & 0.021 & 0.018 & 1000.9 \\
\hline M1032 & 128 & exp06 & 2048bit-ECFP4 & GBM & 0.011 & 1.001 & 0.771 & 0.030 & 0.019 & 500.5 \\
\hline M1033 & 128 & exp07 & 2048bit-ECFP4 & GBM & 0.006 & 0.744 & 0.659 & 0.034 & 0.028 & 1000.2 \\
\hline M1034 & 128 & exp08 & 2048bit-ECFP4 & GBM & 0.003 & 0.685 & 0.621 & 0.029 & 0.026 & 100.1 \\
\hline M1035 & 128 & exp09 & 2048bit-ECFP4 & GBM & 0.007 & 0.892 & 0.706 & 0.020 & 0.013 & 1000.9 \\
\hline M1036 & 128 & exp10 & 2048bit-ECFP4 & GBM & 0.004 & 0.810 & 0.667 & 0.064 & 0.060 & 500.2 \\
\hline M1037 & 128 & exp11 & 2048bit-ECFP4 & GBM & 0.017 & 0.887 & 0.712 & 0.023 & 0.006 & 500.4 \\
\hline M1038 & 128 & exp12 & 2048bit-ECFP4 & GBM & 0.011 & 0.819 & 0.688 & 0.021 & 0.010 & 100.8 \\
\hline M1039 & 128 & exp13 & 2048bit-ECFP4 & GBM & 0.001 & 0.699 & 0.625 & 0.011 & 0.010 & 100.2 \\
\hline M1040 & 128 & exp14 & 2048bit-ECFP4 & GBM & 0.029 & 0.797 & 0.666 & 0.023 & 0.006 & 500.8 \\
\hline M1041 & 128 & exp15 & 2048bit-ECFP4 & GBM & 0.013 & 0.892 & 0.710 & 0.031 & 0.018 & 100.6 \\
\hline M1042 & 128 & exp16 & 2048bit-ECFP4 & GBM & 0.004 & 0.703 & 0.626 & 0.031 & 0.027 & 100.3 \\
\hline M1043 & 128 & exp17 & 2048bit-ECFP4 & GBM & 0.000 & 0.910 & 0.724 & 0.052 & 0.052 & 500.6 \\
\hline M1044 & 128 & exp18 & 2048bit-ECFP4 & GBM & 0.018 & 0.725 & 0.674 & 0.028 & 0.010 & 500.3 \\
\hline M1045 & 128 & exp19 & 2048bit-ECFP4 & GBM & 0.023 & 1.444 & 0.895 & 0.022 & 0.001 & 10000.9 \\
\hline M1046 & 128 & exp01 & 2048bit-ECFP4 & $\mathrm{RF}$ & 0.061 & 0.689 & 0.613 & 0.007 & 0.054 & 500 \\
\hline M1047 & 128 & exp02 & 2048bit-ECFP4 & $\mathrm{RF}$ & 0.000 & 0.838 & 0.689 & 0.007 & 0.007 & 50 \\
\hline M1048 & 128 & exp03 & 2048bit-ECFP4 & $\mathrm{RF}$ & 0.043 & 0.683 & 0.642 & 0.003 & 0.040 & 500 \\
\hline M1049 & 128 & exp04 & 2048bit-ECFP4 & $\mathrm{RF}$ & 0.003 & 0.768 & 0.668 & 0.009 & 0.006 & 10 \\
\hline M1050 & 128 & exp05 & 2048bit-ECFP4 & $\mathrm{RF}$ & 0.000 & 0.836 & 0.719 & 0.013 & 0.013 & 100 \\
\hline M1051 & 128 & exp06 & 2048bit-ECFP4 & $\mathrm{RF}$ & 0.001 & 0.979 & 0.779 & 0.022 & 0.021 & 10 \\
\hline M1052 & 128 & exp07 & 2048bit-ECFP4 & $\mathrm{RF}$ & 0.003 & 0.812 & 0.697 & 0.011 & 0.008 & 100 \\
\hline M1053 & 128 & exp08 & 2048bit-ECFP4 & $\mathrm{RF}$ & 0.003 & 0.864 & 0.736 & 0.004 & 0.001 & 300 \\
\hline M1054 & 128 & exp09 & 2048bit-ECFP4 & $\mathrm{RF}$ & 0.017 & 0.728 & 0.646 & 0.018 & 0.001 & 800 \\
\hline M1055 & 128 & exp10 & 2048bit-ECFP4 & $\mathrm{RF}$ & 0.002 & 0.882 & 0.719 & 0.029 & 0.027 & 100 \\
\hline M1056 & 128 & exp11 & 2048bit-ECFP4 & $\mathrm{RF}$ & 0.015 & 0.929 & 0.739 & 0.009 & 0.006 & 100 \\
\hline M1057 & 128 & exp12 & 2048bit-ECFP4 & $\mathrm{RF}$ & 0.011 & 0.856 & 0.706 & 0.013 & 0.002 & 50 \\
\hline M1058 & 128 & exp13 & 2048bit-ECFP4 & $\mathrm{RF}$ & 0.000 & 0.857 & 0.704 & 0.004 & 0.004 & 10 \\
\hline M1059 & 128 & exp14 & 2048bit-ECFP4 & $\mathrm{RF}$ & 0.029 & 0.729 & 0.638 & 0.008 & 0.021 & 10 \\
\hline M1060 & 128 & exp15 & 2048bit-ECFP4 & $\mathrm{RF}$ & 0.005 & 0.936 & 0.744 & 0.012 & 0.007 & 500 \\
\hline M1061 & 128 & exp16 & 2048bit-ECFP4 & $\mathrm{RF}$ & 0.000 & 0.822 & 0.688 & 0.015 & 0.015 & 100 \\
\hline M1062 & 128 & exp17 & 2048bit-ECFP4 & $\mathrm{RF}$ & 0.001 & 0.890 & 0.722 & 0.037 & 0.036 & 300 \\
\hline M1063 & 128 & exp18 & 2048bit-ECFP4 & $\mathrm{RF}$ & 0.005 & 0.781 & 0.694 & 0.009 & 0.004 & 10 \\
\hline M1064 & 128 & exp19 & 2048bit-ECFP4 & $\mathrm{RF}$ & 0.034 & 1.071 & 0.782 & 0.018 & 0.016 & 10 \\
\hline M1065 & 128 & exp01 & 1024bit-ECFP6 & KNN & 0.000 & 0.833 & 0.687 & 0.029 & 0.029 & 11 distance \\
\hline M1066 & 128 & exp02 & 1024bit-ECFP6 & KNN & 0.000 & 0.789 & 0.672 & 0.013 & 0.013 & 5 uniform \\
\hline M1067 & 128 & exp03 & 1024bit-ECFP6 & KNN & 0.006 & 0.763 & 0.676 & 0.008 & 0.002 & 3 uniform \\
\hline M1068 & 128 & exp04 & 1024bit-ECFP6 & KNN & 0.032 & 0.687 & 0.633 & 0.011 & 0.021 & 3 uniform \\
\hline M1069 & 128 & exp05 & 1024bit-ECFP6 & KNN & 0.000 & 0.797 & 0.697 & 0.023 & 0.023 & 5 distance \\
\hline M1070 & 128 & exp06 & 1024bit-ECFP6 & KNN & 0.001 & 0.763 & 0.649 & 0.016 & 0.015 & 7 uniform \\
\hline M1071 & 128 & exp07 & 1024bit-ECFP6 & KNN & 0.011 & 0.839 & 0.706 & 0.016 & 0.005 & 3 distance \\
\hline M1072 & 128 & exp08 & 1024bit-ECFP6 & KNN & 0.019 & 0.981 & 0.781 & 0.006 & 0.013 & 3 distance \\
\hline
\end{tabular}


Supporting Information (Texts S1-S9, Figures S1-S41 and Tables S1-S12)

\begin{tabular}{|c|c|c|c|c|c|c|c|c|c|c|}
\hline M1073 & 128 & exp09 & 1024bit-ECFP6 & KNN & 0.004 & 1.009 & 0.804 & 0.030 & 0.026 & 1 uniform \\
\hline M1074 & 128 & exp10 & 1024bit-ECFP6 & KNN & 0.001 & 0.734 & 0.623 & 0.039 & 0.038 & 13 uniform \\
\hline M1075 & 128 & exp11 & 1024bit-ECFP6 & KNN & 0.020 & 0.770 & 0.655 & 0.009 & 0.011 & 9 uniform \\
\hline M1076 & 128 & exp12 & 1024bit-ECFP6 & KNN & 0.002 & 0.765 & 0.673 & 0.012 & 0.010 & 15 distance \\
\hline M1077 & 128 & exp13 & 1024bit-ECFP6 & KNN & 0.019 & 0.724 & 0.645 & 0.014 & 0.005 & 5 uniform \\
\hline M1078 & 128 & exp14 & 1024bit-ECFP6 & KNN & 0.031 & 0.920 & 0.767 & 0.017 & 0.014 & 1 uniform \\
\hline M1079 & 128 & exp15 & 1024bit-ECFP6 & KNN & 0.000 & 0.718 & 0.646 & 0.006 & 0.006 & 15 uniform \\
\hline M1080 & 128 & exp16 & 1024bit-ECFP6 & KNN & 0.000 & 0.685 & 0.628 & 0.015 & 0.015 & 13 uniform \\
\hline M1081 & 128 & exp17 & 1024bit-ECFP6 & KNN & 0.009 & 0.925 & 0.739 & 0.029 & 0.020 & 5 distance \\
\hline M1082 & 128 & exp18 & 1024bit-ECFP6 & KNN & 0.000 & 0.800 & 0.683 & 0.012 & 0.012 & 11 distance \\
\hline M1083 & 128 & exp19 & 1024bit-ECFP6 & KNN & 0.035 & 0.854 & 0.695 & 0.014 & 0.021 & 5 uniform \\
\hline M1084 & 128 & exp01 & 1024bit-ECFP6 & SVM & 0.004 & 0.754 & 0.668 & 0.035 & 0.031 & 1000.0005 \\
\hline M1085 & 128 & $\operatorname{exp02}$ & 1024bit-ECFP6 & SVM & 0.011 & 1.136 & 0.797 & 0.018 & 0.007 & 1000.00 .005 \\
\hline M1086 & 128 & exp03 & 1024bit-ECFP6 & SVM & 0.008 & 0.747 & 0.668 & 0.027 & 0.019 & $10000.05 \mathrm{e}-05$ \\
\hline M1087 & 128 & exp04 & 1024bit-ECFP6 & SVM & 0.024 & 0.825 & 0.702 & 0.029 & 0.005 & 50000.00 .0001 \\
\hline M1088 & 128 & exp05 & 1024bit-ECFP6 & SVM & 0.005 & 0.819 & 0.720 & 0.019 & 0.014 & 50.1 \\
\hline M1089 & 128 & exp06 & 1024bit-ECFP6 & SVM & 0.003 & 1.100 & 0.801 & 0.035 & 0.032 & 1000.00 .005 \\
\hline M1090 & 128 & exp07 & 1024bit-ECFP6 & SVM & 0.004 & 0.777 & 0.668 & 0.027 & 0.023 & $10000.01 \mathrm{e}-05$ \\
\hline M1091 & 128 & $\exp 08$ & 1024bit-ECFP6 & SVM & 0.007 & 1.025 & 0.800 & 0.019 & 0.012 & 100000.00 .0001 \\
\hline M1092 & 128 & exp09 & 1024bit-ECFP6 & SVM & 0.012 & 1.034 & 0.776 & 0.020 & 0.008 & 50000.00 .001 \\
\hline M1093 & 128 & $\exp 10$ & 1024bit-ECFP6 & SVM & 0.000 & 0.730 & 0.633 & 0.046 & 0.046 & $5001 \mathrm{e}-05$ \\
\hline M1094 & 128 & exp11 & 1024bit-ECFP6 & SVM & 0.024 & 1.126 & 0.810 & 0.040 & 0.016 & 50000.00 .0005 \\
\hline M1095 & 128 & exp12 & 1024bit-ECFP6 & SVM & 0.014 & 0.646 & 0.602 & 0.027 & 0.013 & $505 \mathrm{e}-05$ \\
\hline M1096 & 128 & exp13 & 1024bit-ECFP6 & SVM & 0.001 & 0.740 & 0.651 & 0.020 & 0.019 & $5005 \mathrm{e}-05$ \\
\hline M1097 & 128 & exp14 & 1024bit-ECFP6 & SVM & 0.037 & 0.833 & 0.721 & 0.014 & 0.023 & 5000.00 .01 \\
\hline M1098 & 128 & exp15 & 1024bit-ECFP6 & SVM & 0.014 & 0.833 & 0.688 & 0.026 & 0.012 & 500.001 \\
\hline M1099 & 128 & exp16 & 1024bit-ECFP6 & SVM & 0.002 & 1.101 & 0.808 & 0.026 & 0.024 & 100000.00 .001 \\
\hline M1100 & 128 & exp17 & 1024bit-ECFP6 & SVM & 0.011 & 0.727 & 0.640 & 0.035 & 0.024 & 10.05 \\
\hline M1101 & 128 & exp18 & 1024bit-ECFP6 & SVM & 0.001 & 0.825 & 0.705 & 0.023 & 0.022 & $100000.01 \mathrm{e}-05$ \\
\hline M1102 & 128 & exp19 & 1024bit-ECFP6 & SVM & 0.012 & 0.890 & 0.723 & 0.017 & 0.005 & 100.01 \\
\hline M1103 & 128 & exp01 & 1024bit-ECFP6 & GBM & 0.004 & 0.921 & 0.731 & 0.030 & 0.026 & 500.9 \\
\hline M1104 & 128 & exp02 & 1024bit-ECFP6 & GBM & 0.007 & 1.022 & 0.751 & 0.022 & 0.015 & 1000.5 \\
\hline M1105 & 128 & exp03 & 1024bit-ECFP6 & GBM & 0.021 & 0.673 & 0.634 & 0.018 & 0.003 & 100.7 \\
\hline M1106 & 128 & exp04 & 1024bit-ECFP6 & GBM & 0.007 & 0.884 & 0.710 & 0.020 & 0.013 & 1000.8 \\
\hline M1107 & 128 & exp05 & 1024bit-ECFP6 & GBM & 0.004 & 0.969 & 0.773 & 0.026 & 0.022 & 3000.8 \\
\hline M1108 & 128 & exp06 & 1024bit-ECFP6 & GBM & 0.002 & 0.979 & 0.760 & 0.025 & 0.023 & 10000.1 \\
\hline M1109 & 128 & exp07 & 1024bit-ECFP6 & GBM & 0.008 & 0.739 & 0.644 & 0.022 & 0.014 & 100.8 \\
\hline M1110 & 128 & exp08 & 1024bit-ECFP6 & GBM & 0.007 & 0.841 & 0.707 & 0.012 & 0.005 & 100.8 \\
\hline M1111 & 128 & exp09 & 1024bit-ECFP6 & GBM & 0.032 & 0.678 & 0.605 & 0.032 & 0.000 & 100.9 \\
\hline M1112 & 128 & exp10 & 1024bit-ECFP6 & GBM & 0.000 & 0.820 & 0.704 & 0.040 & 0.040 & 100.9 \\
\hline M1113 & 128 & exp11 & 1024bit-ECFP6 & GBM & 0.050 & 0.983 & 0.743 & 0.034 & 0.016 & 1000.2 \\
\hline M1114 & 128 & exp12 & 1024bit-ECFP6 & GBM & 0.003 & 0.878 & 0.715 & 0.013 & 0.010 & 500.6 \\
\hline M1115 & 128 & exp13 & 1024bit-ECFP6 & GBM & 0.000 & 0.717 & 0.626 & 0.022 & 0.022 & 100.2 \\
\hline M1116 & 128 & exp14 & 1024bit-ECFP6 & GBM & 0.012 & 0.729 & 0.647 & 0.013 & 0.001 & 100.8 \\
\hline M1117 & 128 & $\exp 15$ & 1024bit-ECFP6 & GBM & 0.002 & 1.134 & 0.839 & 0.017 & 0.015 & 2000.9 \\
\hline
\end{tabular}


Supporting Information (Texts S1-S9, Figures S1-S41 and Tables S1-S12)

\begin{tabular}{|c|c|c|c|c|c|c|c|c|c|c|}
\hline M1118 & 128 & exp16 & 1024bit-ECFP6 & GBM & 0.000 & 0.668 & 0.619 & 0.018 & 0.018 & 100.2 \\
\hline M1119 & 128 & exp17 & 1024bit-ECFP6 & GBM & 0.001 & 0.829 & 0.695 & 0.052 & 0.051 & 500.4 \\
\hline M1120 & 128 & exp18 & 1024bit-ECFP6 & GBM & 0.018 & 0.698 & 0.643 & 0.032 & 0.014 & 100.7 \\
\hline M1121 & 128 & exp19 & 1024bit-ECFP6 & GBM & 0.009 & 1.132 & 0.812 & 0.026 & 0.017 & 2000.3 \\
\hline M1122 & 128 & exp01 & 1024bit-ECFP6 & $\mathrm{RF}$ & 0.019 & 0.737 & 0.660 & 0.013 & 0.006 & 100 \\
\hline M1123 & 128 & $\operatorname{exp02}$ & 1024bit-ECFP6 & $\mathrm{RF}$ & 0.000 & 0.846 & 0.682 & 0.011 & 0.011 & 500 \\
\hline M1124 & 128 & exp03 & 1024bit-ECFP6 & $\mathrm{RF}$ & 0.034 & 0.695 & 0.640 & 0.004 & 0.030 & 10 \\
\hline M1125 & 128 & exp04 & 1024bit-ECFP6 & $\mathrm{RF}$ & 0.003 & 0.762 & 0.657 & 0.007 & 0.004 & 10 \\
\hline M1126 & 128 & exp05 & 1024bit-ECFP6 & $\mathrm{RF}$ & 0.010 & 0.734 & 0.671 & 0.018 & 0.008 & 100 \\
\hline M1127 & 128 & exp06 & 1024bit-ECFP6 & $\mathrm{RF}$ & 0.002 & 0.828 & 0.703 & 0.018 & 0.016 & 500 \\
\hline M1128 & 128 & exp07 & 1024bit-ECFP6 & $\mathrm{RF}$ & 0.006 & 0.803 & 0.694 & 0.010 & 0.004 & 10 \\
\hline M1129 & 128 & $\exp 08$ & 1024bit-ECFP6 & $\mathrm{RF}$ & 0.009 & 0.854 & 0.728 & 0.004 & 0.005 & 10 \\
\hline M1130 & 128 & exp09 & 1024bit-ECFP6 & $\mathrm{RF}$ & 0.040 & 0.694 & 0.627 & 0.016 & 0.024 & 10 \\
\hline M1131 & 128 & exp10 & 1024bit-ECFP6 & $\mathrm{RF}$ & 0.003 & 0.846 & 0.709 & 0.024 & 0.021 & 10 \\
\hline M1132 & 128 & exp11 & 1024bit-ECFP6 & $\mathrm{RF}$ & 0.026 & 0.943 & 0.738 & 0.019 & 0.007 & 50 \\
\hline M1133 & 128 & exp12 & 1024bit-ECFP6 & $\mathrm{RF}$ & 0.003 & 0.796 & 0.683 & 0.010 & 0.007 & 10 \\
\hline M1134 & 128 & exp13 & 1024bit-ECFP6 & $\mathrm{RF}$ & 0.000 & 0.834 & 0.694 & 0.011 & 0.011 & 10 \\
\hline M1135 & 128 & exp14 & 1024bit-ECFP6 & $\mathrm{RF}$ & 0.029 & 0.711 & 0.642 & 0.008 & 0.021 & 200 \\
\hline M1136 & 128 & exp15 & 1024bit-ECFP6 & $\mathrm{RF}$ & 0.001 & 0.861 & 0.717 & 0.012 & 0.011 & 10 \\
\hline M1137 & 128 & exp16 & 1024bit-ECFP6 & $\mathrm{RF}$ & 0.015 & 0.723 & 0.655 & 0.007 & 0.008 & 10 \\
\hline M1138 & 128 & exp17 & 1024bit-ECFP6 & $\mathrm{RF}$ & 0.003 & 0.862 & 0.714 & 0.034 & 0.031 & 50 \\
\hline M1139 & 128 & exp18 & 1024bit-ECFP6 & $\mathrm{RF}$ & 0.003 & 0.788 & 0.704 & 0.009 & 0.006 & 50 \\
\hline M1140 & 128 & exp19 & 1024bit-ECFP6 & $\mathrm{RF}$ & 0.020 & 0.957 & 0.740 & 0.013 & 0.007 & 50 \\
\hline M1141 & 128 & exp01 & 2048bit-ECFP6 & KNN & 0.007 & 0.701 & 0.638 & 0.023 & 0.016 & 13 uniform \\
\hline M1142 & 128 & exp02 & 2048bit-ECFP6 & KNN & 0.001 & 0.765 & 0.651 & 0.022 & 0.021 & 11 uniform \\
\hline M1143 & 128 & exp03 & 2048bit-ECFP6 & KNN & 0.029 & 0.662 & 0.631 & 0.008 & 0.021 & 5 uniform \\
\hline M1144 & 128 & exp04 & 2048bit-ECFP6 & KNN & 0.019 & 1.154 & 0.816 & 0.013 & 0.006 & 1 uniform \\
\hline M1145 & 128 & exp05 & 2048bit-ECFP6 & KNN & 0.000 & 0.792 & 0.696 & 0.021 & 0.021 & 15 distance \\
\hline M1146 & 128 & exp06 & 2048bit-ECFP6 & KNN & 0.000 & 0.788 & 0.676 & 0.027 & 0.027 & 9 uniform \\
\hline M1147 & 128 & exp07 & 2048bit-ECFP6 & KNN & 0.014 & 0.688 & 0.630 & 0.013 & 0.001 & 9 uniform \\
\hline M1148 & 128 & exp08 & 2048bit-ECFP6 & KNN & 0.017 & 1.020 & 0.808 & 0.008 & 0.009 & 3 distance \\
\hline M1149 & 128 & exp09 & 2048bit-ECFP6 & KNN & 0.004 & 0.773 & 0.672 & 0.017 & 0.013 & 3 uniform \\
\hline M1150 & 128 & exp10 & 2048bit-ECFP6 & KNN & 0.000 & 0.738 & 0.633 & 0.040 & 0.040 & 15 uniform \\
\hline M1151 & 128 & exp11 & 2048bit-ECFP6 & KNN & 0.042 & 0.791 & 0.663 & 0.012 & 0.030 & 9 uniform \\
\hline M1152 & 128 & exp12 & 2048bit-ECFP6 & KNN & 0.012 & 0.787 & 0.675 & 0.013 & 0.001 & 5 uniform \\
\hline M1153 & 128 & exp13 & 2048bit-ECFP6 & KNN & 0.001 & 0.807 & 0.680 & 0.013 & 0.012 & 3 uniform \\
\hline M1154 & 128 & exp14 & 2048bit-ECFP6 & KNN & 0.045 & 0.891 & 0.750 & 0.022 & 0.023 & 1 uniform \\
\hline M1155 & 128 & exp15 & 2048bit-ECFP6 & KNN & 0.002 & 0.730 & 0.651 & 0.007 & 0.005 & 15 uniform \\
\hline M1156 & 128 & exp16 & 2048bit-ECFP6 & KNN & 0.004 & 0.705 & 0.628 & 0.014 & 0.010 & 9 uniform \\
\hline M1157 & 128 & exp17 & 2048bit-ECFP6 & KNN & 0.010 & 1.114 & 0.811 & 0.020 & 0.010 & 1 uniform \\
\hline M1158 & 128 & exp18 & 2048bit-ECFP6 & KNN & 0.000 & 0.707 & 0.640 & 0.008 & 0.008 & 11 uniform \\
\hline M1159 & 128 & exp19 & 2048bit-ECFP6 & KNN & 0.004 & 1.255 & 0.879 & 0.010 & 0.006 & 1 distance \\
\hline M1160 & 128 & exp01 & 2048bit-ECFP6 & SVM & 0.000 & 0.904 & 0.729 & 0.078 & 0.078 & $50000.01 \mathrm{e}-05$ \\
\hline M1161 & 128 & exp02 & 2048bit-ECFP6 & SVM & 0.000 & 0.969 & 0.741 & 0.038 & 0.038 & 5000.01 \\
\hline M1162 & 128 & exp03 & 2048bit-ECFP6 & SVM & 0.003 & 0.672 & 0.620 & 0.030 & 0.027 & 500.0001 \\
\hline
\end{tabular}


Supporting Information (Texts S1-S9, Figures S1-S41 and Tables S1-S12)

\begin{tabular}{|c|c|c|c|c|c|c|c|c|c|c|}
\hline M1163 & 128 & exp04 & 2048bit-ECFP6 & SVM & 0.029 & 0.731 & 0.673 & 0.035 & 0.006 & $50000.01 \mathrm{e}-05$ \\
\hline M1164 & 128 & exp05 & 2048bit-ECFP6 & SVM & 0.010 & 0.954 & 0.782 & 0.015 & 0.005 & 50000.00 .0005 \\
\hline M1165 & 128 & exp06 & 2048bit-ECFP6 & SVM & 0.002 & 1.289 & 0.885 & 0.029 & 0.027 & 50000.00 .001 \\
\hline M1166 & 128 & exp07 & 2048bit-ECFP6 & SVM & 0.004 & 0.701 & 0.647 & 0.052 & 0.048 & $5005 \mathrm{e}-05$ \\
\hline M1167 & 128 & exp08 & 2048bit-ECFP6 & SVM & 0.000 & 0.948 & 0.752 & 0.009 & 0.009 & $100000.05 \mathrm{e}-05$ \\
\hline M1168 & 128 & exp09 & 2048bit-ECFP6 & SVM & 0.001 & 1.043 & 0.789 & 0.015 & 0.014 & 1000.05 \\
\hline M1169 & 128 & exp10 & 2048bit-ECFP6 & SVM & 0.000 & 0.762 & 0.653 & 0.072 & 0.072 & 500.0005 \\
\hline M1170 & 128 & exp11 & 2048bit-ECFP6 & SVM & 0.033 & 0.742 & 0.639 & 0.035 & 0.002 & 100.0005 \\
\hline M1171 & 128 & exp12 & 2048bit-ECFP6 & SVM & 0.000 & 0.706 & 0.639 & 0.035 & 0.035 & 50.005 \\
\hline M1172 & 128 & exp13 & 2048bit-ECFP6 & SVM & 0.006 & 0.912 & 0.721 & 0.014 & 0.008 & 1000.00 .001 \\
\hline M1173 & 128 & exp14 & 2048bit-ECFP6 & SVM & 0.048 & 0.770 & 0.669 & 0.015 & 0.033 & 50000.00 .0005 \\
\hline M1174 & 128 & $\exp 15$ & 2048bit-ECFP6 & SVM & 0.003 & 0.893 & 0.735 & 0.063 & 0.060 & $5000.05 \mathrm{e}-05$ \\
\hline M1175 & 128 & exp16 & 2048bit-ECFP6 & SVM & 0.001 & 0.981 & 0.793 & 0.022 & 0.021 & 10000.00 .001 \\
\hline M1176 & 128 & exp17 & 2048bit-ECFP6 & SVM & 0.000 & 1.057 & 0.810 & 0.054 & 0.054 & 50000.00 .005 \\
\hline M1177 & 128 & exp18 & 2048bit-ECFP6 & SVM & 0.033 & 0.670 & 0.642 & 0.027 & 0.006 & 1000.00 .0001 \\
\hline M1178 & 128 & exp19 & 2048bit-ECFP6 & SVM & 0.026 & 0.943 & 0.744 & 0.023 & 0.003 & 100.01 \\
\hline M1179 & 128 & exp01 & 2048bit-ECFP6 & GBM & 0.002 & 0.832 & 0.700 & 0.026 & 0.024 & 3000.1 \\
\hline M1180 & 128 & exp02 & 2048bit-ECFP6 & GBM & 0.000 & 0.981 & 0.748 & 0.036 & 0.036 & 500.9 \\
\hline M1181 & 128 & exp03 & 2048bit-ECFP6 & GBM & 0.004 & 0.724 & 0.636 & 0.021 & 0.017 & 100.9 \\
\hline M1182 & 128 & exp04 & 2048bit-ECFP6 & GBM & 0.068 & 0.613 & 0.596 & 0.013 & 0.055 & 100.3 \\
\hline M1183 & 128 & exp05 & 2048bit-ECFP6 & GBM & 0.000 & 1.012 & 0.793 & 0.019 & 0.019 & 2000.4 \\
\hline M1184 & 128 & exp06 & 2048bit-ECFP6 & GBM & 0.000 & 1.225 & 0.865 & 0.028 & 0.028 & 10000.4 \\
\hline M1185 & 128 & exp07 & 2048bit-ECFP6 & GBM & 0.002 & 0.863 & 0.716 & 0.034 & 0.032 & 500.7 \\
\hline M1186 & 128 & exp08 & 2048bit-ECFP6 & GBM & 0.003 & 0.669 & 0.614 & 0.016 & 0.013 & 100.1 \\
\hline M1187 & 128 & exp09 & 2048bit-ECFP6 & GBM & 0.002 & 1.054 & 0.799 & 0.018 & 0.016 & 3000.8 \\
\hline M1188 & 128 & exp10 & 2048bit-ECFP6 & GBM & 0.003 & 0.861 & 0.707 & 0.045 & 0.042 & 2000.1 \\
\hline M1189 & 128 & exp11 & 2048bit-ECFP6 & GBM & 0.028 & 1.026 & 0.759 & 0.026 & 0.002 & 500.7 \\
\hline M1190 & 128 & exp12 & 2048bit-ECFP6 & GBM & 0.002 & 0.667 & 0.610 & 0.019 & 0.017 & 100.1 \\
\hline M1191 & 128 & exp13 & 2048bit-ECFP6 & GBM & 0.001 & 0.711 & 0.627 & 0.011 & 0.010 & 100.2 \\
\hline M1192 & 128 & exp14 & 2048bit-ECFP6 & GBM & 0.033 & 0.682 & 0.614 & 0.015 & 0.018 & 500.2 \\
\hline M1193 & 128 & exp15 & 2048bit-ECFP6 & GBM & 0.002 & 1.152 & 0.824 & 0.036 & 0.034 & 10000.9 \\
\hline M1194 & 128 & exp16 & 2048bit-ECFP6 & GBM & 0.006 & 0.966 & 0.781 & 0.027 & 0.021 & 9000.3 \\
\hline M1195 & 128 & exp17 & 2048bit-ECFP6 & GBM & 0.000 & 0.842 & 0.689 & 0.048 & 0.048 & 1000.3 \\
\hline M1196 & 128 & exp18 & 2048bit-ECFP6 & GBM & 0.006 & 0.664 & 0.607 & 0.043 & 0.037 & 100.1 \\
\hline M1197 & 128 & exp19 & 2048bit-ECFP6 & GBM & 0.007 & 1.201 & 0.853 & 0.017 & 0.010 & 2000.3 \\
\hline M1198 & 128 & exp01 & 2048bit-ECFP6 & $\mathrm{RF}$ & 0.007 & 0.790 & 0.688 & 0.014 & 0.007 & 700 \\
\hline M1199 & 128 & exp02 & 2048bit-ECFP6 & $\mathrm{RF}$ & 0.002 & 0.818 & 0.673 & 0.017 & 0.015 & 1000 \\
\hline M1200 & 128 & exp03 & 2048bit-ECFP6 & $\mathrm{RF}$ & 0.026 & 0.714 & 0.644 & 0.005 & 0.020 & 10 \\
\hline M1201 & 128 & exp04 & 2048bit-ECFP6 & $\mathrm{RF}$ & 0.000 & 0.808 & 0.688 & 0.007 & 0.007 & 10 \\
\hline M1202 & 128 & exp05 & 2048bit-ECFP6 & $\mathrm{RF}$ & 0.002 & 0.829 & 0.724 & 0.014 & 0.012 & 200 \\
\hline M1203 & 128 & exp06 & 2048bit-ECFP6 & $\mathrm{RF}$ & 0.000 & 0.956 & 0.753 & 0.025 & 0.025 & 900 \\
\hline M1204 & 128 & exp07 & 2048bit-ECFP6 & $\mathrm{RF}$ & 0.004 & 0.805 & 0.695 & 0.018 & 0.014 & 200 \\
\hline M1205 & 128 & exp08 & 2048bit-ECFP6 & $\mathrm{RF}$ & 0.002 & 0.883 & 0.743 & 0.004 & 0.002 & 100 \\
\hline M1206 & 128 & exp09 & 2048bit-ECFP6 & $\mathrm{RF}$ & 0.012 & 0.757 & 0.664 & 0.013 & 0.001 & 300 \\
\hline M1207 & 128 & $\exp 10$ & 2048bit-ECFP6 & $\mathrm{RF}$ & 0.005 & 0.873 & 0.719 & 0.023 & 0.018 & 50 \\
\hline
\end{tabular}


Supporting Information (Texts S1-S9, Figures S1-S41 and Tables S1-S12)

\begin{tabular}{|l|l|l|l|l|l|l|l|l|l|l|}
\hline M1208 & 128 & exp11 & 2048bit-ECFP6 & RF & 0.018 & 0.907 & 0.716 & 0.016 & 0.002 & 50 \\
\hline M1209 & 128 & exp12 & 2048bit-ECFP6 & RF & 0.006 & 0.835 & 0.698 & 0.006 & 0.000 & 100 \\
\hline M1210 & 128 & exp13 & 2048bit-ECFP6 & RF & 0.003 & 0.877 & 0.720 & 0.009 & 0.006 & 50 \\
\hline M1211 & 128 & exp14 & 2048bit-ECFP6 & RF & 0.024 & 0.731 & 0.660 & 0.008 & 0.016 & 50 \\
\hline M1212 & 128 & exp15 & 2048bit-ECFP6 & RF & 0.002 & 0.888 & 0.733 & 0.015 & 0.013 & 800 \\
\hline M1213 & 128 & exp16 & 2048bit-ECFP6 & RF & 0.003 & 0.771 & 0.693 & 0.010 & 0.007 & 100 \\
\hline M1214 & 128 & exp17 & 2048bit-ECFP6 & RF & 0.000 & 0.840 & 0.701 & 0.039 & 0.039 & 300 \\
\hline M1215 & 128 & exp18 & 2048bit-ECFP6 & RF & 0.005 & 0.778 & 0.705 & 0.010 & 0.005 & 600 \\
\hline M1216 & 128 & exp19 & 2048bit-ECFP6 & RF & 0.010 & 0.986 & 0.770 & 0.022 & 0.012 & 10 \\
\hline
\end{tabular}

* Optimal parameters for each machine-learning methods as follows

(1) KNN: two parameters are the number of nearest neighbors $(\mathrm{K})$ and weighting schemes (uniform weight or distance-dependent weight) respectively

(2) SVM: two parameters are the penalty parameter $\mathrm{C}$ and kernel parameter gamma respectively

(3) RF: one parameter is the number of decision trees

(4) GBM: two parameters are the number of decision trees and the learning rate 
Table S7. 64 average regression models derived from 1216 individual regression "models after applying Y-randomization test" (Table S6) by averaging over 19 data-splitting schemes.

\begin{tabular}{|c|c|c|c|c|c|c|c|c|}
\hline Model & FS & FP & method & $\mathbf{R}^{2}$ (holdout test set) & MSE(holdout test set) & MAE(holdout test set) & $R^{2}(C V)$ & $\Delta \mathbf{R}^{2}$ \\
\hline AM01 & full & 1024bit-ECFP4 & KNN & $0.015(0.017)$ & $0.830(0.202)$ & $0.688(0.074)$ & $0.018(0.009)$ & $0.015(0.009)$ \\
\hline AM02 & full & 1024bit-ECFP4 & SVM & $0.022(0.046)$ & $0.988(0.314)$ & $0.757(0.125)$ & $0.019(0.008)$ & $0.025(0.043)$ \\
\hline AM03 & full & 1024bit-ECFP4 & GBM & $0.015(0.014)$ & $0.894(0.188)$ & $0.715(0.073)$ & $0.017(0.007)$ & $0.014(0.010)$ \\
\hline AM04 & full & 1024bit-ECFP4 & $\mathrm{RF}$ & $0.015(0.016)$ & $0.809(0.098)$ & $0.689(0.040)$ & $0.013(0.009)$ & $0.015(0.012)$ \\
\hline AM05 & full & 2048bit-ECFP4 & KNN & $0.014(0.013)$ & $0.831(0.144)$ & $0.695(0.062)$ & $0.019(0.009)$ & $0.015(0.008)$ \\
\hline AM06 & full & 2048bit-ECFP4 & SVM & $0.026(0.047)$ & $0.931(0.252)$ & $0.732(0.106)$ & $0.019(0.008)$ & $0.026(0.044)$ \\
\hline AM07 & full & 2048bit-ECFP4 & GBM & $0.012(0.011)$ & $0.845(0.145)$ & $0.696(0.067)$ & $0.017(0.007)$ & $0.013(0.008)$ \\
\hline AM08 & full & 2048bit-ECFP4 & $\mathrm{RF}$ & $0.015(0.018)$ & $0.813(0.101)$ & $0.692(0.043)$ & $0.012(0.008)$ & $0.015(0.013)$ \\
\hline AM09 & full & 1024bit-ECFP6 & KNN & $0.024(0.022)$ & $0.825(0.148)$ & $0.692(0.063)$ & $0.019(0.007)$ & $0.018(0.016)$ \\
\hline AM10 & full & 1024bit-ECFP6 & SVM & $0.009(0.009)$ & $0.897(0.233)$ & $0.721(0.100)$ & $0.018(0.008)$ & $0.011(0.007)$ \\
\hline AM11 & full & 1024bit-ECFP6 & GBM & $0.011(0.013)$ & $0.816(0.118)$ & $0.685(0.054)$ & $0.018(0.006)$ & $0.015(0.010)$ \\
\hline AM12 & full & 1024bit-ECFP6 & $\mathrm{RF}$ & $0.014(0.016)$ & $0.777(0.078)$ & $0.672(0.034)$ & $0.011(0.007)$ & $0.013(0.010)$ \\
\hline AM13 & full & 2048bit-ECFP6 & KNN & $0.024(0.027)$ & $0.794(0.143)$ & $0.676(0.065)$ & $0.020(0.009)$ & $0.019(0.021)$ \\
\hline AM14 & full & 2048bit-ECFP6 & SVM & $0.016(0.026)$ & $0.902(0.221)$ & $0.724(0.095)$ & $0.018(0.008)$ & $0.016(0.024)$ \\
\hline AM15 & full & 2048bit-ECFP6 & GBM & $0.009(0.010)$ & $0.830(0.167)$ & $0.684(0.067)$ & $0.020(0.009)$ & $0.014(0.011)$ \\
\hline AM16 & full & 2048bit-ECFP6 & $\mathrm{RF}$ & $0.012(0.013)$ & $0.797(0.087)$ & $0.682(0.036)$ & $0.013(0.010)$ & $0.012(0.010)$ \\
\hline AM17 & 512 & 1024bit-ECFP4 & KNN & $0.015(0.016)$ & $0.860(0.205)$ & $0.702(0.076)$ & $0.018(0.009)$ & $0.015(0.009)$ \\
\hline AM18 & 512 & 1024bit-ECFP4 & SVM & $0.023(0.044)$ & $0.941(0.343)$ & $0.732(0.132)$ & $0.021(0.014)$ & $0.029(0.041)$ \\
\hline AM19 & 512 & 1024bit-ECFP4 & GBM & $0.012(0.010)$ & $0.881(0.162)$ & $0.709(0.066)$ & $0.018(0.009)$ & $0.013(0.010)$ \\
\hline AM20 & 512 & 1024bit-ECFP4 & $\mathrm{RF}$ & $0.015(0.016)$ & $0.813(0.099)$ & $0.690(0.041)$ & $0.013(0.008)$ & $0.014(0.012)$ \\
\hline AM21 & 512 & 2048bit-ECFP4 & KNN & $0.013(0.016)$ & $0.788(0.095)$ & $0.675(0.044)$ & $0.017(0.009)$ & $0.015(0.013)$ \\
\hline AM22 & 512 & 2048bit-ECFP4 & SVM & $0.026(0.043)$ & $0.936(0.309)$ & $0.732(0.126)$ & $0.020(0.009)$ & $0.031(0.038)$ \\
\hline AM23 & 512 & 2048bit-ECFP4 & GBM & $0.013(0.014)$ & $0.842(0.143)$ & $0.696(0.068)$ & $0.019(0.008)$ & $0.015(0.011)$ \\
\hline AM24 & 512 & 2048bit-ECFP4 & $\mathrm{RF}$ & $0.014(0.018)$ & $0.816(0.102)$ & $0.692(0.043)$ & $0.012(0.008)$ & $0.016(0.014)$ \\
\hline AM25 & 512 & 1024bit-ECFP6 & KNN & $0.019(0.022)$ & $0.803(0.108)$ & $0.681(0.052)$ & $0.018(0.008)$ & $0.019(0.017)$ \\
\hline AM26 & 512 & 1024bit-ECFP6 & SVM & $0.014(0.017)$ & $1.071(0.415)$ & $0.777(0.146)$ & $0.020(0.007)$ & $0.018(0.013)$ \\
\hline AM27 & 512 & 1024bit-ECFP6 & GBM & $0.015(0.018)$ & $0.800(0.101)$ & $0.680(0.046)$ & $0.020(0.007)$ & $0.018(0.012)$ \\
\hline AM28 & 512 & 1024bit-ECFP6 & $\mathrm{RF}$ & $0.013(0.016)$ & $0.779(0.075)$ & $0.673(0.033)$ & $0.010(0.006)$ & $0.013(0.012)$ \\
\hline AM29 & 512 & 2048bit-ECFP6 & KNN & $0.013(0.016)$ & $0.801(0.100)$ & $0.681(0.049)$ & $0.017(0.008)$ & $0.016(0.012)$ \\
\hline AM30 & 512 & 2048bit-ECFP6 & SVM & $0.014(0.018)$ & $0.945(0.292)$ & $0.742(0.123)$ & $0.021(0.007)$ & $0.020(0.011)$ \\
\hline AM31 & 512 & 2048bit-ECFP6 & GBM & $0.011(0.015)$ & $0.845(0.183)$ & $0.689(0.072)$ & $0.021(0.010)$ & $0.021(0.011)$ \\
\hline AM32 & 512 & 2048bit-ECFP6 & $\mathrm{RF}$ & $0.011(0.012)$ & $0.803(0.084)$ & $0.686(0.033)$ & $0.013(0.009)$ & $0.012(0.010)$ \\
\hline AM33 & 256 & 1024bit-ECFP4 & KNN & $0.011(0.014)$ & $0.794(0.094)$ & $0.678(0.042)$ & $0.017(0.007)$ & $0.015(0.011)$ \\
\hline AM34 & 256 & 1024bit-ECFP4 & SVM & $0.015(0.025)$ & $0.896(0.193)$ & $0.721(0.089)$ & $0.022(0.011)$ & $0.024(0.020)$ \\
\hline AM35 & 256 & 1024bit-ECFP4 & GBM & $0.011(0.014)$ & $0.894(0.112)$ & $0.722(0.052)$ & $0.022(0.012)$ & $0.018(0.013)$ \\
\hline AM36 & 256 & 1024bit-ECFP4 & $\mathrm{RF}$ & $0.015(0.016)$ & $0.807(0.095)$ & $0.686(0.040)$ & $0.012(0.009)$ & $0.015(0.013)$ \\
\hline AM37 & 256 & 2048bit-ECFP4 & KNN & $0.008(0.010)$ & $0.835(0.178)$ & $0.691(0.069)$ & $0.017(0.007)$ & $0.014(0.007)$ \\
\hline
\end{tabular}


Supporting Information (Texts S1-S9, Figures S1-S41 and Tables S1-S12)

\begin{tabular}{|c|c|c|c|c|c|c|c|c|}
\hline AM38 & 256 & 2048bit-ECFP4 & SVM & $0.021(0.032)$ & $0.918(0.254)$ & $0.725(0.103)$ & $0.022(0.011)$ & $0.025(0.029)$ \\
\hline AM39 & 256 & 2048bit-ECFP4 & GBM & $0.014(0.013)$ & $0.822(0.135)$ & $0.686(0.060)$ & $0.023(0.011)$ & $0.015(0.011)$ \\
\hline AM40 & 256 & 2048bit-ECFP4 & $\mathrm{RF}$ & $0.017(0.021)$ & $0.822(0.105)$ & $0.695(0.045)$ & $0.011(0.008)$ & $0.017(0.017)$ \\
\hline AM41 & 256 & 1024bit-ECFP6 & KNN & $0.015(0.020)$ & $0.781(0.090)$ & $0.671(0.046)$ & $0.016(0.008)$ & $0.015(0.016)$ \\
\hline AM42 & 256 & 1024bit-ECFP6 & SVM & $0.023(0.039)$ & $0.846(0.198)$ & $0.695(0.085)$ & $0.021(0.008)$ & $0.026(0.035)$ \\
\hline AM43 & 256 & 1024bit-ECFP6 & GBM & $0.010(0.012)$ & $0.848(0.166)$ & $0.696(0.073)$ & $0.021(0.008)$ & $0.015(0.011)$ \\
\hline AM44 & 256 & 1024bit-ECFP6 & $\mathrm{RF}$ & $0.013(0.016)$ & $0.788(0.080)$ & $0.678(0.035)$ & $0.011(0.006)$ & $0.013(0.011)$ \\
\hline AM45 & 256 & 2048bit-ECFP6 & KNN & $0.013(0.019)$ & $0.841(0.180)$ & $0.695(0.071)$ & $0.017(0.008)$ & $0.018(0.013)$ \\
\hline AM46 & 256 & 2048bit-ECFP6 & SVM & $0.009(0.015)$ & $0.889(0.181)$ & $0.718(0.085)$ & $0.024(0.011)$ & $0.022(0.013)$ \\
\hline AM47 & 256 & 2048bit-ECFP6 & GBM & $0.007(0.009)$ & $0.873(0.186)$ & $0.706(0.079)$ & $0.023(0.010)$ & $0.018(0.012)$ \\
\hline AM48 & 256 & 2048bit-ECFP6 & $\mathrm{RF}$ & $0.009(0.010)$ & $0.815(0.086)$ & $0.694(0.038)$ & $0.012(0.008)$ & $0.010(0.009)$ \\
\hline AM49 & 128 & 1024bit-ECFP4 & KNN & $0.007(0.008)$ & $0.850(0.171)$ & $0.697(0.071)$ & $0.017(0.008)$ & $0.013(0.008)$ \\
\hline AM50 & 128 & 1024bit-ECFP4 & SVM & $0.018(0.028)$ & $0.848(0.164)$ & $0.699(0.072)$ & $0.024(0.017)$ & $0.028(0.025)$ \\
\hline AM51 & 128 & 1024bit-ECFP4 & GBM & $0.013(0.013)$ & $0.883(0.204)$ & $0.708(0.078)$ & $0.022(0.013)$ & $0.017(0.014)$ \\
\hline AM52 & 128 & 1024bit-ECFP4 & $\mathrm{RF}$ & $0.012(0.013)$ & $0.827(0.095)$ & $0.695(0.039)$ & $0.013(0.010)$ & $0.012(0.011)$ \\
\hline AM53 & 128 & 2048bit-ECFP4 & KNN & $0.015(0.028)$ & $0.802(0.114)$ & $0.680(0.058)$ & $0.018(0.010)$ & $0.019(0.021)$ \\
\hline AM54 & 128 & 2048bit-ECFP4 & SVM & $0.014(0.015)$ & $0.812(0.118)$ & $0.687(0.054)$ & $0.024(0.011)$ & $0.019(0.014)$ \\
\hline AM55 & 128 & 2048bit-ECFP4 & GBM & $0.012(0.011)$ & $0.838(0.189)$ & $0.687(0.073)$ & $0.026(0.013)$ & $0.018(0.016)$ \\
\hline AM56 & 128 & 2048bit-ECFP4 & $\mathrm{RF}$ & $0.012(0.017)$ & $0.839(0.097)$ & $0.701(0.045)$ & $0.013(0.009)$ & $0.015(0.014)$ \\
\hline AM57 & 128 & 1024bit-ECFP6 & KNN & $0.010(0.012)$ & $0.808(0.091)$ & $0.687(0.051)$ & $0.017(0.009)$ & $0.016(0.009)$ \\
\hline AM58 & 128 & 1024bit-ECFP6 & SVM & $0.010(0.009)$ & $0.877(0.153)$ & $0.715(0.065)$ & $0.027(0.008)$ & $0.019(0.010)$ \\
\hline AM59 & 128 & 1024bit-ECFP6 & GBM & $0.010(0.012)$ & $0.858(0.146)$ & $0.703(0.065)$ & $0.025(0.010)$ & $0.017(0.012)$ \\
\hline AM60 & 128 & 1024bit-ECFP6 & $\mathrm{RF}$ & $0.012(0.012)$ & $0.804(0.075)$ & $0.687(0.033)$ & $0.013(0.007)$ & $0.012(0.008)$ \\
\hline AM61 & 128 & 2048bit-ECFP6 & KNN & $0.011(0.014)$ & $0.835(0.168)$ & $0.696(0.075)$ & $0.016(0.008)$ & $0.015(0.010)$ \\
\hline AM62 & 128 & 2048bit-ECFP6 & SVM & $0.010(0.015)$ & $0.876(0.158)$ & $0.719(0.071)$ & $0.035(0.020)$ & $0.029(0.024)$ \\
\hline AM63 & 128 & 2048bit-ECFP6 & GBM & $0.009(0.016)$ & $0.881(0.190)$ & $0.713(0.088)$ & $0.026(0.011)$ & $0.025(0.014)$ \\
\hline AM64 & 128 & 2048bit-ECFP6 & $\mathrm{RF}$ & $0.007(0.008)$ & $0.834(0.070)$ & $0.705(0.032)$ & $0.014(0.008)$ & $0.012(0.009)$ \\
\hline
\end{tabular}


Table S8. Nineteen individual regression models (from Table S3) used to construct the consensus model (CM01)

\begin{tabular}{|c|c|c|c|c|c|c|c|c|c|c|}
\hline Model & FS & $\operatorname{Exp}$ & FP & Method & $\begin{array}{c}\mathbf{R}^{2} \\
\text { (holdout } \\
\text { test) }\end{array}$ & $\begin{array}{c}\text { MSE } \\
\text { (holdout } \\
\text { test) }\end{array}$ & $\begin{array}{c}\text { MAE } \\
\text { (holdout } \\
\text { test) }\end{array}$ & $\begin{array}{c}\mathbf{R}^{2} \\
(\mathbf{C V})\end{array}$ & $\Delta \mathbf{R}^{2}$ & $\begin{array}{l}\text { Parameters* } \\
\text { from CV }\end{array}$ \\
\hline M1160 & 128 & exp01 & 2048bit-ECFP6 & SVM & 0.717 & 0.198 & 0.337 & 0.675 & 0.042 & 50.05 \\
\hline M1047 & 128 & exp02 & 2048bit-ECFP4 & $\mathrm{RF}$ & 0.696 & 0.225 & 0.360 & 0.653 & 0.043 & 200 \\
\hline M0839 & 256 & exp03 & 2048bit-ECFP6 & KNN & 0.687 & 0.212 & 0.351 & 0.650 & 0.037 & 7 distance \\
\hline M0555 & 512 & exp04 & 2048bit-ECFP6 & SVM & 0.657 & 0.226 & 0.365 & 0.659 & 0.002 & 50.01 \\
\hline M0404 & 512 & exp05 & 2048bit-ECFP4 & SVM & 0.695 & 0.203 & 0.346 & 0.669 & 0.026 & 50.05 \\
\hline M0709 & 256 & exp06 & 2048bit-ECFP4 & SVM & 0.666 & 0.235 & 0.370 & 0.662 & 0.004 & 100.05 \\
\hline M0273 & full & exp07 & 2048bit-ECFP6 & GBM & 0.616 & 0.266 & 0.391 & 0.607 & 0.009 & 3000.1 \\
\hline M0255 & full & exp08 & 2048bit-ECFP6 & SVM & 0.652 & 0.236 & 0.370 & 0.698 & 0.046 & 100.01 \\
\hline M1016 & 128 & exp09 & 2048bit-ECFP4 & SVM & 0.613 & 0.265 & 0.388 & 0.627 & 0.014 & 50.05 \\
\hline M0713 & 256 & $\exp 10$ & 2048bit-ECFP4 & SVM & 0.663 & 0.236 & 0.369 & 0.653 & 0.010 & 50.05 \\
\hline M1170 & 128 & exp11 & 2048bit-ECFP6 & SVM & 0.692 & 0.217 & 0.350 & 0.646 & 0.046 & 50.05 \\
\hline M0544 & 512 & $\exp 12$ & 2048bit-ECFP6 & KNN & 0.643 & 0.243 & 0.378 & 0.636 & 0.007 & 5 distance \\
\hline M1039 & 128 & exp13 & 2048bit-ECFP4 & GBM & 0.696 & 0.219 & 0.361 & 0.658 & 0.038 & 3000.1 \\
\hline M0660 & 256 & exp14 & 1024bit-ECFP4 & GBM & 0.609 & 0.280 & 0.408 & 0.652 & 0.043 & 1000.3 \\
\hline M0642 & 256 & $\exp 15$ & 1024bit-ECFP4 & SVM & 0.698 & 0.213 & 0.332 & 0.649 & 0.048 & 50.05 \\
\hline M0263 & full & exp16 & 2048bit-ECFP6 & SVM & 0.653 & 0.232 & 0.365 & 0.658 & 0.005 & 100.01 \\
\hline M1157 & 128 & $\exp 17$ & 2048bit-ECFP6 & KNN & 0.633 & 0.271 & 0.394 & 0.649 & 0.016 & 3 distance \\
\hline M0664 & 256 & exp18 & 1024bit-ECFP4 & GBM & 0.662 & 0.228 & 0.372 & 0.615 & 0.047 & 2000.1 \\
\hline M0570 & 512 & exp19 & 2048bit-ECFP6 & SVM & 0.669 & 0.227 & 0.359 & 0.661 & 0.008 & 50.05 \\
\hline
\end{tabular}

* Optimal parameters for each machine-learning methods as follows

(1) KNN: two parameters are the number of nearest neighbors $(\mathrm{K})$ and weighting schemes (uniform weight or distance-dependent weight) respectively

(2) SVM: two parameters are the penalty parameter $\mathrm{C}$ and kernel parameter gamma respectively

(3) GBM: two parameters are the number of decision trees and the learning rate 
Table S9. Prediction results for three species (human, mouse, and "unknown") based on the best average model (AM14)

\begin{tabular}{|c|c|c|c|c|}
\hline species & Number of samples & $\begin{array}{c}\mathbf{R}^{2} \text { (external } \\
\text { test set) }\end{array}$ & $\begin{array}{c}\text { MSE(external } \\
\text { test set) }\end{array}$ & $\begin{array}{c}\text { MAE(external } \\
\text { test set) }\end{array}$ \\
\hline human & 55 & 0.070 & 0.094 & 0.261 \\
\hline mouse & 14 & 0.348 & 0.268 & 0.460 \\
\hline unknown & 1 & 0.000 & 0.009 & 0.095 \\
\hline all & 70 & 0.848 & 0.127 & 0.298 \\
\hline
\end{tabular}

Notes: (1) "number of samples" is the total number of samples with specific species that are separated from the external test set; (2) "all" refers to all the species; (2) $\mathrm{R}^{2}$ (Human) is quite low, because the distribution of experimental $\mathrm{HD}_{50}$ values in the external test set is quite narrow (1.5 to 2.5 in term of LogHD 50 for human, however, the small MSE and MAE values indicate that predicted $\operatorname{LogHD}_{50}$ is still close to the experimental $\operatorname{LogHD} \mathrm{D}_{50}$, which can be observed from Figure $\mathbf{S 3 3}$; (3) $\mathrm{R}^{2}$ (unknown) is zero due to the fact that only one sample with "Unknown" species is existed in the external test. 
Table S10. Prediction results for each species based on 19 individual models of AM14

\begin{tabular}{|c|c|c|c|c|}
\hline species & number of samples & $\begin{array}{c}\mathbf{R}^{\mathbf{2}} \text { (holdout test } \\
\text { set) }\end{array}$ & $\begin{array}{c}\text { MSE(holdout } \\
\text { test set) }\end{array}$ & $\begin{array}{c}\text { MAE(holdout } \\
\text { test set) }\end{array}$ \\
\hline human & 1250 & 0.472 & 0.218 & 0.349 \\
\hline sheep & 591 & 0.685 & 0.171 & 0.313 \\
\hline mouse & 383 & 0.520 & 0.188 & 0.338 \\
\hline unknown & 326 & 0.587 & 0.348 & 0.449 \\
\hline rabbit & 332 & 0.746 & 0.369 & 0.463 \\
\hline pig & 76 & 0.164 & 0.382 & 0.473 \\
\hline rat & 62 & 0.482 & 0.325 & 0.494 \\
\hline bovine & 26 & 0.212 & 0.074 & 0.201 \\
\hline dog & 13 & 0.580 & 0.120 & 0.339 \\
\hline all & 3059 & 0.648 & 0.240 & 0.368 \\
\hline
\end{tabular}

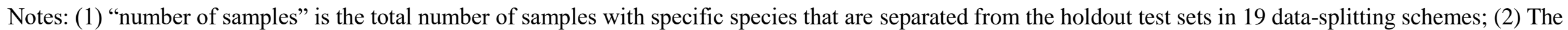

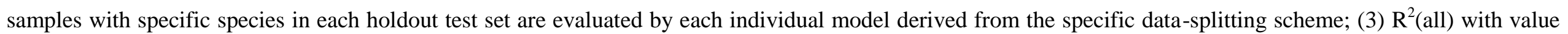

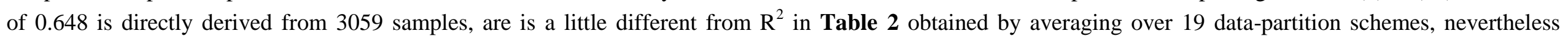
MSE(All) and MAE(all) are the same as the counterparts in Table 2. 
Table S11. 155 fragments from recursive fragmentation of Dataset-CV \& Dataset-Holdout-Test (805 compounds)

\begin{tabular}{|c|c|c|c|c|c|c|}
\hline Fragment name & $\begin{array}{c}\text { Predicted } \\
\text { LogHD }_{50}\end{array}$ & $\begin{array}{l}\text { Average } \\
\text { similarity } \\
\text { for } \mathrm{AD}^{*}\end{array}$ & $\begin{array}{c}\text { Fragment } \\
\text { size }^{*}\end{array}$ & $\begin{array}{l}\text { Fragment } \\
\text { efficiency }\end{array}$ & $\begin{array}{c}\text { Parent } \\
\text { molecule } \\
\text { size* }\end{array}$ & SMILE string \\
\hline 001_frag_22810 & 1.3072 & 0.21 & 29 & -0.063 & 96 & $\begin{array}{l}\mathrm{C}(\mathrm{O})[\mathrm{C} @ @ \mathrm{H}](\mathrm{C}[\mathrm{C} @ @ \mathrm{H}](\mathrm{C}[\mathrm{C} @ @ \mathrm{H}] 1[\mathrm{C} @ \mathrm{H}]([\mathrm{C} @ @ \mathrm{H}](\mathrm{C}[\mathrm{C} @ @ \mathrm{H}](\mathrm{O} 1)[\mathrm{C} @ \\
@ \mathrm{H}](\mathrm{C}[\mathrm{C} @ @ \mathrm{H}](\mathrm{CCC} / \mathrm{C}(=\mathrm{C} / \mathrm{CC}) / \mathrm{C}) \mathrm{C}) \mathrm{O}) \mathrm{O}) \mathrm{O}) \mathrm{O}) \mathrm{O}\end{array}$ \\
\hline 002_frag_31017 & 1.4071 & 0.36 & 30 & -0.066 & 79 & $\begin{array}{l}\mathrm{C}=\mathrm{CCC} / \mathrm{C}=\mathrm{C} / \mathrm{C}=\mathrm{C} / \mathrm{C}=\mathrm{C} / \mathrm{CC} / \mathrm{C}=\mathrm{C} /[\mathrm{C} @ @ \mathrm{H}](\mathrm{O})[\mathrm{C} @ \mathrm{H}](\mathrm{O})[\mathrm{C} @ @ \mathrm{H}] 1 \mathrm{O}[\mathrm{C} @ \mathrm{H}](\mathrm{C}[ \\
\mathrm{C} @ \mathrm{H}]([\mathrm{C} @ \mathrm{H}] 1 \mathrm{O}) \mathrm{O})[\mathrm{C} @ \mathrm{H}](\mathrm{O}) \mathrm{CO}\end{array}$ \\
\hline 003_frag_355 & 1.5258 & 0.58 & 30 & -0.071 & 71 & $\begin{array}{l}\mathrm{C} 1[\mathrm{C} @ @ \mathrm{H}](\mathrm{CC} 2=\mathrm{CC}[\mathrm{C} @ @ \mathrm{H}] 3[\mathrm{C} @ @ \mathrm{H}]([\mathrm{C} @] 2(\mathrm{C} 1) \mathrm{C}) \mathrm{CC}[\mathrm{C} @] 1([\mathrm{C} @ @ \mathrm{H}] 3 \mathrm{C} \\
[\mathrm{C} @ \mathrm{H}] 2[\mathrm{C} @ @ \mathrm{H}] 1[\mathrm{C} @ @ \mathrm{H}]([\mathrm{C} @ @] 1(\mathrm{O} 2) \mathrm{OC}[\mathrm{C} @ @ \mathrm{H}](\mathrm{CC} 1) \mathrm{C}) \mathrm{C}) \mathrm{C}) \mathrm{O}\end{array}$ \\
\hline 004_frag_16 & 1.5383 & 0.48 & 30 & -0.072 & 61 & $\begin{array}{l}\mathrm{C} 1[\mathrm{C} @ @ \mathrm{H}](\mathrm{C}[\mathrm{C} @ @ \mathrm{H}] 2[\mathrm{C} @](\mathrm{C} 1)([\mathrm{C} @ @ \mathrm{H}] 1[\mathrm{C} @ @ \mathrm{H}](\mathrm{CC} 2)[\mathrm{C} @ @ \mathrm{H}] 2[\mathrm{C} @]( \\
\mathrm{CC} 1)([\mathrm{C} @ @ \mathrm{H}] 1[\mathrm{C} @ \mathrm{H}](\mathrm{C} 2) \mathrm{O}[\mathrm{C} @ @] 2([\mathrm{C} @ \mathrm{H}] 1 \mathrm{C}) \mathrm{CC}[\mathrm{C} @ \mathrm{H}](\mathrm{CO} 2) \mathrm{C}) \mathrm{C}) \mathrm{C}) \mathrm{O}\end{array}$ \\
\hline 005_frag_9433 & 1.5519 & 0.22 & 29 & -0.075 & 83 & $\begin{array}{l}\mathrm{OC}[\mathrm{C} @ @ \mathrm{H}](\mathrm{C} / \mathrm{C}=\mathrm{C} / \mathrm{CC}[\mathrm{C} @ @ \mathrm{H}](\mathrm{C}[\mathrm{C} @ @ \mathrm{H}]([\mathrm{C} @ \mathrm{H}](\mathrm{C}[\mathrm{C} @ @ \mathrm{H}]([\mathrm{C} @ \mathrm{H}](\mathrm{CCC} \\
\mathrm{O}) \mathrm{O}) \mathrm{C}) \mathrm{O}) \mathrm{O}) \mathrm{C}) \mathrm{OS}(\mathrm{O})(\mathrm{O}) \mathrm{O}\end{array}$ \\
\hline 006_frag_5330 & 1.4094 & 0.22 & 26 & -0.076 & 94 & $\mathrm{C}(\mathrm{O})[\mathrm{C} @ @ \mathrm{H}](\mathrm{C} / \mathrm{C}=\mathrm{C} /[\mathrm{C} @ @ \mathrm{H}](\mathrm{O}) \mathrm{C} / \mathrm{C}=\mathrm{C} / \mathrm{CCCC}[\mathrm{C} @ @ \mathrm{H}](\mathrm{CCCCCCCC}) \mathrm{O}) \mathrm{O}$ \\
\hline 007_frag_4971 & 1.5378 & 0.18 & 28 & -0.077 & 95 & $\begin{array}{l}\mathrm{C}(=\mathrm{ClCC} / \mathrm{C}=\mathrm{C} / \mathrm{C}=\mathrm{C} / \mathrm{C}=\mathrm{C} / \mathrm{CC} / \mathrm{C}=\mathrm{C} / \mathrm{C}[\mathrm{C} @ \mathrm{H}](\mathrm{O})[\mathrm{C} @ @ \mathrm{H}] 1 \mathrm{O}[\mathrm{C} @ \mathrm{H}](\mathrm{CC}[\mathrm{C} @ \mathrm{H}] 1 \\
\mathrm{O}) \mathrm{CO}) / \mathrm{C}=\mathrm{C}\end{array}$ \\
\hline 008_frag_31 & 1.6121 & 0.5 & 29 & -0.078 & 72 & $\begin{array}{l}\mathrm{C} 1 \mathrm{CCC} 2=\mathrm{CC}[\mathrm{C} @ @ \mathrm{H}] 3[\mathrm{C} @ @ \mathrm{H}]([\mathrm{C} @] 2(\mathrm{C} 1) \mathrm{C}) \mathrm{CC}[\mathrm{C} @] 1([\mathrm{C} @ @ \mathrm{H}] 3 \mathrm{C}[\mathrm{C} @ \mathrm{H}] 2 \\
[\mathrm{C} @ @ \mathrm{H}] 1[\mathrm{C} @ @ \mathrm{H}]([\mathrm{C} @ @] 1(\mathrm{O} 2) \mathrm{OC}[\mathrm{C} @ @ \mathrm{H}](\mathrm{CC} 1) \mathrm{C}) \mathrm{C}) \mathrm{C}\end{array}$ \\
\hline 009_frag_3494 & 1.6164 & 0.53 & 29 & -0.078 & 134 & $\begin{array}{l}\mathrm{C} 1[\mathrm{C} @ @ \mathrm{H}](\mathrm{C}[\mathrm{C} @ @ \mathrm{H}] 2[\mathrm{C} @](\mathrm{C} 1)([\mathrm{C} @ @ \mathrm{H}] 1[\mathrm{C} @ @ \mathrm{H}](\mathrm{CC} 2)[\mathrm{C} @ @ \mathrm{H}] 2[\mathrm{C} @]( \\
\mathrm{CC} 1)([\mathrm{C} @ \mathrm{H}](\mathrm{CC} 2)[\mathrm{C} @ \mathrm{H}](\mathrm{C}) \mathrm{CCC}(=\mathrm{O}) \mathrm{NCC}) \mathrm{C}) \mathrm{C}) \mathrm{O}\end{array}$ \\
\hline 010_frag_4743 & 1.7485 & 0.33 & 29 & -0.084 & 136 & $\begin{array}{l}\mathrm{C} 1[\mathrm{C} @ @ \mathrm{H}](\mathrm{C}[\mathrm{C} @ @ \mathrm{H}] 2[\mathrm{C} @](\mathrm{C} 1)([\mathrm{C} @ @ \mathrm{H}] 1[\mathrm{C} @ @ \mathrm{H}]([\mathrm{C} @ @ \mathrm{H}](\mathrm{C} 2) \mathrm{O})[\mathrm{C} @ \\
@ \mathrm{H}] 2[\mathrm{C} @](\mathrm{CC} 1)([\mathrm{C} @ \mathrm{H}](\mathrm{CC} 2)[\mathrm{C} @ \mathrm{H}](\mathrm{C}) \mathrm{CCC}(=\mathrm{O}) \mathrm{NC}) \mathrm{C}) \mathrm{C}) \mathrm{O}\end{array}$ \\
\hline 011_frag_191 & 1.7667 & 0.3 & 29 & -0.085 & 98 & $\begin{array}{l}\mathrm{C} 1[\mathrm{C} @ @ \mathrm{H}](\mathrm{C}([\mathrm{C} @ \mathrm{H}] 2[\mathrm{C} @](\mathrm{C} 1)([\mathrm{C} @ @ \mathrm{H}] 1[\mathrm{C} @ @](\mathrm{CC} 2)([\mathrm{C} @] 2(\mathrm{C}(=\mathrm{CC} 1)[\mathrm{C} \\
@ \mathrm{H}] 1[\mathrm{C} @ @ \mathrm{H}](\mathrm{CC} 2) \mathrm{CCC}[\mathrm{C} @ @ \mathrm{H}] 1 \mathrm{C}) \mathrm{C}) \mathrm{C}) \mathrm{C})(\mathrm{C}) \mathrm{C}) \mathrm{O}\end{array}$ \\
\hline 012_frag_1018 & 1.7179 & 0.56 & 28 & -0.086 & 58 & {$[\mathrm{C} @ \mathrm{H}](\mathrm{C}(=\mathrm{O}) \mathrm{NCC})([\mathrm{N}+](\mathrm{C})(\mathrm{C}) \mathrm{C}) \mathrm{CCCCNC}(=\mathrm{O}) \mathrm{CCCCCCCCCCC}$} \\
\hline 013_frag_2142 & 1.5702 & 0.79 & 25 & -0.088 & 55 & $\operatorname{CCCCCCCNC}(=\mathrm{O})[\mathrm{C} @ \mathrm{H}](\mathrm{CCCNC}(\mathrm{N}) \mathrm{N}) \mathrm{NC}(=\mathrm{O}) \mathrm{CCCC}$ \\
\hline 014_frag_253 & 1.7751 & 0.19 & 28 & -0.089 & 80 & $\begin{array}{l}\mathrm{S}(=\mathrm{O})(=\mathrm{O})(\mathrm{c} 1 \mathrm{c} 2 \mathrm{c}(\mathrm{ccc} 1) \mathrm{c}(\mathrm{ccc} 2) \mathrm{N}(\mathrm{C}) \mathrm{C}) \mathrm{NCCO}[\mathrm{C} @ @ \mathrm{H}] 1 \mathrm{CC}[\mathrm{C} @ @ \mathrm{H}](\mathrm{O}) \mathrm{O}[\mathrm{C} @ \mathrm{H} \\
] 1 \mathrm{C}\end{array}$ \\
\hline 015_frag_811 & 1.9017 & 0.39 & 30 & -0.089 & 86 & $\begin{array}{l}\mathrm{C}(\mathrm{C}(=\mathrm{O}) \mathrm{N}[\mathrm{C} @ \mathrm{H}](\mathrm{C}(=\mathrm{O}) \mathrm{N}[\mathrm{C} @ @ \mathrm{H}](\mathrm{C}(=\mathrm{O}) \mathrm{O}) \mathrm{CC}(\mathrm{C}) \mathrm{C}) \mathrm{Cc} 1 \mathrm{c}[\mathrm{nH}] \mathrm{c} 2 \mathrm{c} 1 \mathrm{cccc} 2) \mathrm{CC}( \\
\mathrm{C}) \mathrm{C}\end{array}$ \\
\hline 016_frag_1185 & 1.5186 & 0.22 & 23 & -0.092 & 54 & $\begin{array}{l}\mathrm{C}(\mathrm{C}) \mathrm{CCCCO}[\mathrm{C} @ \mathrm{H}] 1[\mathrm{C} @ @ \mathrm{H}](\mathrm{C}[\mathrm{C} @ @ \mathrm{H}](\mathrm{O} 1) \mathrm{C}) \mathrm{O}[\mathrm{C} @ @ \mathrm{H}] 1[\mathrm{C} @ \mathrm{H}]([\mathrm{C} @ @ \mathrm{H}] \\
([\mathrm{C} @ \mathrm{H}](\mathrm{CO} 1) \mathrm{O}) \mathrm{O}) \mathrm{O}\end{array}$ \\
\hline 017_frag_2204 & 1.8983 & 0.66 & 29 & -0.092 & 59 & $\mathrm{~N}[\mathrm{C} @ @ \mathrm{H}](\mathrm{NCCC}[\mathrm{C} @ @ \mathrm{H}](\mathrm{C}(=\mathrm{O}) \mathrm{NCCCC}) \mathrm{NC}(=\mathrm{O}) \mathrm{CCCCCCCCCCC}) \mathrm{N}$ \\
\hline
\end{tabular}


Supporting Information (Texts S1-S9, Figures S1-S41 and Tables S1-S12)

\begin{tabular}{|c|c|c|c|c|c|c|}
\hline 018_frag_992 & 1.5962 & 0.16 & 24 & -0.093 & 60 & $\begin{array}{l}{[\mathrm{C} @ @ \mathrm{H}] 1(\mathrm{CCC}[\mathrm{C} @ @ \mathrm{H}](\mathrm{O} 1) \mathrm{C}) \mathrm{O}[\mathrm{C} @ \mathrm{H}] 1 \mathrm{CO}[\mathrm{C} @ @ \mathrm{H}]([\mathrm{C} @ \mathrm{H}](\mathrm{C} 1) \mathrm{O}[\mathrm{C} @ @ \mathrm{H}]} \\
1 \mathrm{O}[\mathrm{C} @ \mathrm{H}](\mathrm{C}[\mathrm{C} @ @ \mathrm{H}] 1 \mathrm{O}) \mathrm{C}) \mathrm{CO}\end{array}$ \\
\hline 019_frag_208 & 1.8552 & 0.19 & 28 & -0.093 & 81 & $\begin{array}{l}\mathrm{C}(=\mathrm{O})(\mathrm{OCC} 1=\mathrm{c} 2 \mathrm{c}(=\mathrm{C} 3[\mathrm{C} @ @ \mathrm{H}] 1 \mathrm{C}=\mathrm{CC}=\mathrm{C} 3) \mathrm{cccc} 2) \mathrm{NCCO}[\mathrm{C} @ @ \mathrm{H}] 1 \mathrm{CCCO}[\mathrm{C} \\
@ \mathrm{H}] 1 \mathrm{C}\end{array}$ \\
\hline 020_frag_6403 & 1.7493 & 0.17 & 26 & -0.094 & 56 & $\begin{array}{l}\mathrm{C}(\mathrm{C}(\mathrm{C}) \mathrm{C}) \mathrm{CO}[\mathrm{C} @ \mathrm{H}] 1[\mathrm{C} @ @ \mathrm{H}](\mathrm{C}[\mathrm{C} @ @ \mathrm{H}](\mathrm{O} 1)[\mathrm{C} @ @ \mathrm{H}](\mathrm{CO}) \mathrm{O}) \mathrm{O}[\mathrm{C} @ @ \mathrm{H}] 1[\mathrm{C} \\
@ \mathrm{H}](\mathrm{C}[\mathrm{C} @ \mathrm{H}](\mathrm{CO} 1) \mathrm{OC}) \mathrm{OC}\end{array}$ \\
\hline 021_frag_990 & 1.9574 & 0.43 & 29 & -0.094 & 78 & $\mathrm{NCC}[\mathrm{C} @ @ \mathrm{H}](\mathrm{C}=\mathrm{O}) \mathrm{NC}(=\mathrm{O})[\mathrm{C} @ \mathrm{H}](\mathrm{NC}(=\mathrm{O}) \mathrm{CCCCCCCCCCCCC}) \mathrm{CCN}$ \\
\hline 022_frag_2294 & 1.6212 & 0.17 & 24 & -0.095 & 174 & $\begin{array}{l}\mathrm{C} 1 \mathrm{CC}[\mathrm{C} @ @ \mathrm{H}](\mathrm{OC} 1) \mathrm{O}[\mathrm{C} @ @ \mathrm{H}] 1 \mathrm{CC}[\mathrm{C} @ \mathrm{H}](\mathrm{O}[\mathrm{C} @ \mathrm{H}] 1 \mathrm{OC}[\mathrm{C} @ @ \mathrm{H}] 1 \mathrm{C}[\mathrm{C} @ @ \mathrm{H} \\
]([\mathrm{C} @ \mathrm{H}](\mathrm{CO} 1) \mathrm{O}) \mathrm{O}) \mathrm{C}\end{array}$ \\
\hline 023_frag_452 & 1.6954 & 0.17 & 25 & -0.095 & 62 & $\begin{array}{l}\mathrm{O}[\mathrm{C} @ \mathrm{H}] 1[\mathrm{C} @ @ \mathrm{H}](\mathrm{C}[\mathrm{C} @ \mathrm{H}](\mathrm{CO} 1) \mathrm{O}[\mathrm{C} @ @ \mathrm{H}] 1 \mathrm{CC}[\mathrm{C} @ \mathrm{H}]([\mathrm{C} @ @ \mathrm{H}](\mathrm{O} 1) \mathrm{CO}) \mathrm{O} \\
) \mathrm{O}[\mathrm{C} @ \mathrm{H}] 1 \mathrm{CCC}[\mathrm{C} @ @ \mathrm{H}](\mathrm{O} 1) \mathrm{C}\end{array}$ \\
\hline 024_frag_0 & 1.9008 & 0.33 & 28 & -0.095 & 62 & $\mathrm{C}(=\mathrm{O}) \mathrm{Nc} 1 \mathrm{cc}(\operatorname{cc}(\mathrm{c} 1 \mathrm{SCCN}) \mathrm{NC}(=\mathrm{O}) \mathrm{CCCCNC}(\mathrm{N}) \mathrm{N}) \mathrm{C}(\mathrm{F})(\mathrm{F}) \mathrm{F}$ \\
\hline 025_frag_0 & 1.9029 & 0.36 & 28 & -0.095 & 70 & $\mathrm{C}(=\mathrm{O}) \mathrm{Nc} 1 \operatorname{cc}(\operatorname{cc}(\mathrm{c} 1 \mathrm{SCCN}) \mathrm{NC}(=\mathrm{O}) \mathrm{CCCCNC}(\mathrm{N}) \mathrm{N}) \mathrm{C}(\mathrm{C})(\mathrm{C}) \mathrm{C}$ \\
\hline 026_frag_2328 & 1.671 & 0.24 & 24 & -0.097 & 101 & $\begin{array}{l}\mathrm{C} 1[\mathrm{C} @ @ \mathrm{H}](\mathrm{CCO}[\mathrm{C} @ @ \mathrm{H}] 1 \mathrm{C}) \mathrm{O}[\mathrm{C} @ \mathrm{H}] 1[\mathrm{C} @ @ \mathrm{H}]([\mathrm{C} @ \mathrm{H}]([\mathrm{C} @ @ \mathrm{H}]([\mathrm{C} @ \mathrm{H}](\mathrm{O} \\
1) \mathrm{COS}(\mathrm{O})(\mathrm{O}) \mathrm{O}) \mathrm{O}) \mathrm{OC}) \mathrm{O}\end{array}$ \\
\hline 027_frag_7233 & 1.7287 & 0.18 & 25 & -0.097 & 55 & $\begin{array}{l}\mathrm{C} 1 \mathrm{C}[\mathrm{C} @ @ \mathrm{H}]([\mathrm{C} @ @ \mathrm{H}] 2[\mathrm{C} @ \mathrm{H}](\mathrm{C} 1)[\mathrm{C} @ @ \mathrm{H}] 1[\mathrm{C} @ @ \mathrm{H}](\mathrm{CC} 2)[\mathrm{C} @ @ \mathrm{H}] 2[\mathrm{C} @] \\
(\mathrm{CC} 1)([\mathrm{C} @ \mathrm{H}]([\mathrm{C} @ \mathrm{H}]([\mathrm{C} @ \mathrm{H}] 2 \mathrm{O}) \mathrm{O})[\mathrm{C} @ \mathrm{H}](\mathrm{C}) \mathrm{CC}) \mathrm{C}) \mathrm{O}\end{array}$ \\
\hline 028_frag_194 & 2.0197 & 0.29 & 29 & -0.098 & 62 & $\begin{array}{l}\mathrm{C} 1 \mathrm{C}[\mathrm{C} @ @ \mathrm{H}]([\mathrm{C} @ @ \mathrm{H}] 2[\mathrm{C} @](\mathrm{C} 1)([\mathrm{C} @ @ \mathrm{H}] 1[\mathrm{C} @ @](\mathrm{CC} 2)([\mathrm{C} @] 2([\mathrm{C} @ @ \mathrm{H}]( \\
\mathrm{CC} 1)[\mathrm{C} @ \mathrm{H}] 1[\mathrm{C} @](\mathrm{CC} 2)(\mathrm{C}) \mathrm{CC}[\mathrm{C} @ \mathrm{H}] 1 \mathrm{C}(=\mathrm{C}) \mathrm{C}) \mathrm{C}) \mathrm{C}) \mathrm{C}) \mathrm{C}\end{array}$ \\
\hline 029_frag_3218 & 1.6967 & 0.43 & 24 & -0.099 & 54 & $\mathrm{C}(\mathrm{C}(=\mathrm{O}) \mathrm{NCCCNCCC}) \mathrm{CCCCNC}(=\mathrm{O}) \mathrm{CCCCCC}$ \\
\hline 030_frag_437 & 1.9209 & 0.24 & 27 & -0.1 & 98 & $\begin{array}{l}\mathrm{C} 1[\mathrm{C} @ @ \mathrm{H}](\mathrm{C}([\mathrm{C} @ \mathrm{H}] 2[\mathrm{C} @](\mathrm{C} 1)([\mathrm{C} @ @ \mathrm{H}] 1[\mathrm{C} @ @](\mathrm{CC} 2)([\mathrm{C} @] 2([\mathrm{C} @ @ \mathrm{H}](\mathrm{C} \\
\mathrm{C} 1)[\mathrm{C} @ \mathrm{H}] 1[\mathrm{C} @ @ \mathrm{H}](\mathrm{CC} 2) \mathrm{CCC} 1) \mathrm{C}) \mathrm{C}) \mathrm{C})(\mathrm{C}) \mathrm{C}) \mathrm{O}\end{array}$ \\
\hline 031_frag_449 & 1.7242 & 0.16 & 24 & -0.101 & 107 & $\begin{array}{l}{[\mathrm{C} @ \mathrm{H}] 1(\mathrm{CCCCO} 1) \mathrm{O}[\mathrm{C} @ \mathrm{H}] 1[\mathrm{C} @ @ \mathrm{H}](\mathrm{CCO}[\mathrm{C} @ @ \mathrm{H}] 1 \mathrm{C}) \mathrm{O}[\mathrm{C} @ @ \mathrm{H}] 1 \mathrm{CC}[\mathrm{C} @} \\
\mathrm{H}]([\mathrm{C} @ \mathrm{H}](\mathrm{O} 1) \mathrm{CO}) \mathrm{O}\end{array}$ \\
\hline 032_frag_139 & 1.9875 & 0.29 & 27 & -0.103 & 72 & $\begin{array}{l}\mathrm{C} 1[\mathrm{C} @ @ \mathrm{H}]([\mathrm{C} @ @ \mathrm{H}]([\mathrm{C} @ @ \mathrm{H}] 2[\mathrm{C} @](\mathrm{C} 1)([\mathrm{C} @ @ \mathrm{H}] 1 \mathrm{C}(=\mathrm{CC} 2)[\mathrm{C} @] 2([\mathrm{C} @] 3( \\
\mathrm{CC} 1)[\mathrm{C} @ \mathrm{H}]([\mathrm{C} @ \mathrm{H}](\mathrm{C} 2) \mathrm{OC} 3=\mathrm{O}) \mathrm{C}(=\mathrm{C}) \mathrm{C}) \mathrm{C}) \mathrm{C}) \mathrm{C}) \mathrm{O}\end{array}$ \\
\hline 033_frag_409 & 1.7042 & 0.16 & 23 & -0.104 & 73 & $\begin{array}{l}\mathrm{C} 1 \mathrm{CC}[\mathrm{C} @ \mathrm{H}](\mathrm{CO} 1) \mathrm{O}[\mathrm{C} @ @ \mathrm{H}] 1 \mathrm{CC}[\mathrm{C} @ @ \mathrm{H}]([\mathrm{C} @ \mathrm{H}](\mathrm{O} 1) \mathrm{C}) \mathrm{O}[\mathrm{C} @ @ \mathrm{H}] 1 \mathrm{OC}[\mathrm{C} \\
@ @ \mathrm{H}]([\mathrm{C} @ \mathrm{H}] 1 \mathrm{O}) \mathrm{CO}\end{array}$ \\
\hline 034_frag_2835 & 1.7839 & 0.17 & 24 & -0.104 & 93 & $\begin{array}{l}{[\mathrm{C} @ \mathrm{H}] 1([\mathrm{C} @ \mathrm{H}](\mathrm{CCO}[\mathrm{C} @ \mathrm{H}] 1 \mathrm{COS}(\mathrm{O})(\mathrm{O}) \mathrm{O}) \mathrm{O}[\mathrm{C} @ @ \mathrm{H}] 1 \mathrm{C}[\mathrm{C} @ @ \mathrm{H}]([\mathrm{C} @ \mathrm{H}]([\mathrm{C}} \\
@ @ \mathrm{H}](\mathrm{O} 1) \mathrm{C}) \mathrm{O}) \mathrm{OC}) \mathrm{O}\end{array}$ \\
\hline 035_frag_950 & 1.8548 & 0.18 & 25 & -0.104 & 83 & $\begin{array}{l}{[\mathrm{C} @ @ \mathrm{H}] 1([\mathrm{C} @ @ \mathrm{H}]([\mathrm{C} @ \mathrm{H}]([\mathrm{C} @ @ \mathrm{H}](\mathrm{OC} 1) \mathrm{O}[\mathrm{C} @ @ \mathrm{H}] 1 \mathrm{C}[\mathrm{C} @ @ \mathrm{H}](\mathrm{O}[\mathrm{C} @ \mathrm{H}](} \\
\mathrm{C} 1) \mathrm{O}) \mathrm{C}) \mathrm{O}) \mathrm{O}[\mathrm{C} @ \mathrm{H}] 1 \mathrm{CCC}[\mathrm{C} @ @ \mathrm{H}](\mathrm{O} 1) \mathrm{C}) \mathrm{O}\end{array}$ \\
\hline 036_frag_1058 & 1.7949 & 0.16 & 24 & -0.105 & 111 & $\begin{array}{l}{[\mathrm{C} @ @ \mathrm{H}] 1(\mathrm{CCC}[\mathrm{C} @ \mathrm{H}](\mathrm{O} 1) \mathrm{CO}) \mathrm{O}[\mathrm{C} @ @ \mathrm{H}] 1[\mathrm{C} @ \mathrm{H}]([\mathrm{C} @ @ \mathrm{H}](\mathrm{OCC} 1) \mathrm{O}[\mathrm{C} @ @ ~} \\
\mathrm{H}] 1 \mathrm{CCCO}[\mathrm{C} @ \mathrm{H}] 1 \mathrm{C}) \mathrm{O}\end{array}$ \\
\hline 037_frag_848 & 1.8055 & 0.16 & 24 & -0.105 & 63 & $\begin{array}{l}\mathrm{C} 1[\mathrm{C} @ @ \mathrm{H}]([\mathrm{C} @ \mathrm{H}]([\mathrm{C} @ \mathrm{H}](\mathrm{CO} 1) \mathrm{O}[\mathrm{C} @ \mathrm{H}] 1 \mathrm{CCC}[\mathrm{C} @ \mathrm{H}](\mathrm{O} 1) \mathrm{CO}) \mathrm{O}) \mathrm{O}[\mathrm{C} @ \mathrm{H}] 1 \\
\mathrm{CCC}[\mathrm{C} @ @ \mathrm{H}](\mathrm{O} 1) \mathrm{C}\end{array}$ \\
\hline 038_frag_2582 & 2.2497 & 0.31 & 30 & -0.105 & 89 & $\mathrm{~N}([\mathrm{C} @ @ \mathrm{H}](\mathrm{C}(=\mathrm{O}) \mathrm{N}[\mathrm{C} @ @ \mathrm{H}](\mathrm{CCCCN}) \mathrm{C}(=\mathrm{O}) \mathrm{N}[\mathrm{C} @ @ \mathrm{H}](\mathrm{C}=\mathrm{O}) \mathrm{CCCCN}) \mathrm{CCCC}$ \\
\hline
\end{tabular}


Supporting Information (Texts S1-S9, Figures S1-S41 and Tables S1-S12)

\begin{tabular}{|c|c|c|c|c|c|c|}
\hline & & & & & & $\mathrm{N}) \mathrm{C}(=\mathrm{O}) \mathrm{C}$ \\
\hline 039_frag_241 & 1.7482 & 0.19 & 23 & -0.106 & 65 & $\begin{array}{l}\mathrm{C} 1 \mathrm{C}[\mathrm{C} @ \mathrm{H}](\mathrm{COC} 1) \mathrm{O}[\mathrm{C} @ \mathrm{H}] 1[\mathrm{C} @ @ \mathrm{H}]([\mathrm{C} @ \mathrm{H}]([\mathrm{C} @ @ \mathrm{H}]([\mathrm{C} @ \mathrm{H}](\mathrm{O} 1) \mathrm{C}) \mathrm{O}) \mathrm{O}) \mathrm{O} \\
{[\mathrm{C} @ \mathrm{H}] 1 \mathrm{CCCCO} 1}\end{array}$ \\
\hline 040_frag_456 & 1.9069 & 0.27 & 25 & -0.107 & 76 & $\begin{array}{l}\mathrm{O}[\mathrm{C} @ @ \mathrm{H}] 1 \mathrm{C}[\mathrm{C} @ @ \mathrm{H}]([\mathrm{C} @ \mathrm{H}]([\mathrm{C} @ @ \mathrm{H}](\mathrm{O} 1) \mathrm{C}) \mathrm{OCCNC}(=\mathrm{O}) \mathrm{CCCCCCCCC}=\mathrm{C} \\
) \mathrm{O}\end{array}$ \\
\hline 041_frag_652 & 1.767 & 0.16 & 23 & -0.108 & 85 & $\begin{array}{l}\mathrm{C} 1 \mathrm{CC}[\mathrm{C} @ \mathrm{H}]([\mathrm{C} @ @ \mathrm{H}](\mathrm{O} 1) \mathrm{C}) \mathrm{O}[\mathrm{C} @ \mathrm{H}] 1 \mathrm{C}[\mathrm{C} @ \mathrm{H}](\mathrm{CCO} 1) \mathrm{O}[\mathrm{C} @ @ \mathrm{H}] 1 \mathrm{OC}[\mathrm{C} @ \mathrm{H} \\
]([\mathrm{C} @ @ \mathrm{H}] 1 \mathrm{O}) \mathrm{CO}\end{array}$ \\
\hline 042_frag_696 & 1.7679 & 0.16 & 23 & -0.108 & 82 & $\begin{array}{l}{[\mathrm{C} @ \mathrm{H}] 1([\mathrm{C} @ \mathrm{H}]([\mathrm{C} @ @ \mathrm{H}](\mathrm{CCO} 1) \mathrm{O}[\mathrm{C} @ \mathrm{H}] 1 \mathrm{OC}[\mathrm{C} @ @ \mathrm{H}](\mathrm{C} 1) \mathrm{CO}) \mathrm{O}[\mathrm{C} @ @ \mathrm{H}] 1} \\
\mathrm{OC}[\mathrm{C} @ @ \mathrm{H}]([\mathrm{C} @ \mathrm{H}] 1 \mathrm{O}) \mathrm{C}) \mathrm{C}\end{array}$ \\
\hline 043_frag_3651 & 1.8496 & 0.61 & 24 & -0.108 & 56 & $\mathrm{~N}[\mathrm{C} @ \mathrm{H}](\mathrm{NCCC}[\mathrm{C} @ @ \mathrm{H}](\mathrm{C}(=\mathrm{O}) \mathrm{NCCC}) \mathrm{NC}(=\mathrm{O}) \mathrm{CCCCCCC}) \mathrm{N}$ \\
\hline 044_frag_1675 & 1.8347 & 0.17 & 23 & -0.112 & 108 & $\begin{array}{l}\mathrm{O}[\mathrm{C} @ \mathrm{H}] 1 \mathrm{C}[\mathrm{C} @ \mathrm{H}](\mathrm{CCO} 1) \mathrm{O}[\mathrm{C} @ \mathrm{H}] 1[\mathrm{C} @ @ \mathrm{H}]([\mathrm{C} @ \mathrm{H}](\mathrm{C}[\mathrm{C} @ \mathrm{H}](\mathrm{O} 1) \mathrm{COS}(\mathrm{O})(\mathrm{O} \\
) \mathrm{O}) \mathrm{OC}) \mathrm{O}\end{array}$ \\
\hline 045_frag_733 & 1.6213 & 0.18 & 20 & -0.113 & 54 & $\begin{array}{l}\mathrm{C} 1 \mathrm{CC}[\mathrm{C} @ @ \mathrm{H}]([\mathrm{C} @ \mathrm{H}](\mathrm{O} 1) \mathrm{CO}) \mathrm{O}[\mathrm{C} @ @ \mathrm{H}] 1[\mathrm{C} @ @ \mathrm{H}](\mathrm{C}[\mathrm{C} @ \mathrm{H}]([\mathrm{C} @ @ \mathrm{H}](\mathrm{O} 1) \\
\mathrm{C}) \mathrm{OC}=\mathrm{O}) \mathrm{O}\end{array}$ \\
\hline 046_frag_575 & 1.77 & 0.48 & 22 & -0.113 & 52 & $\operatorname{CCCCCCNC}(=\mathrm{O}) \operatorname{CCCCCNC}(=\mathrm{O}) \mathrm{CCCCC}$ \\
\hline 047_frag_228 & 1.8556 & 0.2 & 23 & -0.113 & 98 & $\begin{array}{l}\mathrm{C} 1[\mathrm{C} @ @ \mathrm{H}](\mathrm{CCCO} 1) \mathrm{O}[\mathrm{C} @ \mathrm{H}] 1[\mathrm{C} @ @ \mathrm{H}]([\mathrm{C} @ @ \mathrm{H}]([\mathrm{C} @ \mathrm{H}]([\mathrm{C} @ @ \mathrm{H}](\mathrm{O} 1) \mathrm{C}) \mathrm{O}[ \\
\mathrm{C} @ \mathrm{H}] 1 \mathrm{CCCCO} 1) \mathrm{O}) \mathrm{O}\end{array}$ \\
\hline 048_frag_668 & 1.8574 & 0.18 & 23 & -0.113 & 82 & $\begin{array}{l}\mathrm{O}[\mathrm{C} @ \mathrm{H}] 1 \mathrm{CC}[\mathrm{C} @ @ \mathrm{H}](\mathrm{CO} 1) \mathrm{O}[\mathrm{C} @ @ \mathrm{H}] 1 \mathrm{C}[\mathrm{C} @ @ \mathrm{H}]([\mathrm{C} @ \mathrm{H}]([\mathrm{C} @ @ \mathrm{H}](\mathrm{O} 1) \mathrm{CO} \\
\mathrm{S}(\mathrm{O})(\mathrm{O}) \mathrm{O}) \mathrm{O}) \mathrm{OC}\end{array}$ \\
\hline 049_frag_0 & 1.943 & 0.38 & 24 & -0.113 & 68 & $\mathrm{C}(=\mathrm{O}) \mathrm{Nc} 1 \mathrm{cc}(\operatorname{cc}(\mathrm{c} 1 \mathrm{SCCNC}(\mathrm{N}) \mathrm{N}) \mathrm{NC}(\mathrm{N}) \mathrm{N}) \mathrm{C}(\mathrm{C})(\mathrm{C}) \mathrm{C}$ \\
\hline 050_frag_48 & 2.0092 & 0.27 & 25 & -0.113 & 75 & $\begin{array}{l}\mathrm{O}[\mathrm{C} @ @ \mathrm{H}] 1[\mathrm{C} @ @ \mathrm{H}]([\mathrm{C} @ @ \mathrm{H}]([\mathrm{C} @ \mathrm{H}]([\mathrm{C} @ @ \mathrm{H}](\mathrm{O} 1) \mathrm{C}) \mathrm{OCCNC}(=\mathrm{O}) \mathrm{c} 1 \mathrm{ccc}(\mathrm{cc} \\
1) \mathrm{N}(=\mathrm{O})=\mathrm{O}) \mathrm{O}) \mathrm{O}\end{array}$ \\
\hline 051_frag_1304 & 1.8762 & 0.35 & 23 & -0.114 & 53 & {$[\mathrm{C} @ @ \mathrm{H}] 1(\mathrm{CN}(\mathrm{CN} 1 \mathrm{CCCCCCCC}) \mathrm{CCCCC}) \mathrm{CCC}(=\mathrm{O}) \mathrm{N}$} \\
\hline 052_frag_48 & 2.0301 & 0.26 & 25 & -0.114 & 75 & $\begin{array}{l}\mathrm{O}[\mathrm{C} @ @ \mathrm{H}] 1[\mathrm{C} @ @ \mathrm{H}]([\mathrm{C} @ @ \mathrm{H}]([\mathrm{C} @ \mathrm{H}]([\mathrm{C} @ @ \mathrm{H}](\mathrm{O} 1) \mathrm{C}) \mathrm{OCCNC}(=\mathrm{O}) \mathrm{COc} 1 \mathrm{ccc} \\
(\mathrm{cc} 1) \mathrm{Cl}) \mathrm{O}) \mathrm{O}\end{array}$ \\
\hline 053_frag_195 & 1.3952 & 0.51 & 17 & -0.115 & 48 & $\mathrm{O}=\mathrm{C}(\mathrm{c} 1 \mathrm{cc}[\mathrm{n}+](\mathrm{cc} 1) \mathrm{CCCCCCCC}) \mathrm{O}$ \\
\hline 054_frag_777 & 1.6475 & 0.17 & 20 & -0.115 & 50 & C(C)CCCO[C@H]1[C@@H](C[C@H](CO[C@@H]2CCCCO2)O1)O \\
\hline 055_frag_872 & 1.9886 & 0.46 & 24 & -0.116 & 54 & $\mathrm{~N}([\mathrm{C} @ \mathrm{H}](\mathrm{C}(=\mathrm{O}) \mathrm{NCCc} 1 \operatorname{ccccc} 1) \mathrm{CCC}) \mathrm{C}(=\mathrm{O}) \mathrm{CCCCCC}$ \\
\hline 056_frag_48 & 1.996 & 0.25 & 24 & -0.116 & 74 & $\begin{array}{l}\mathrm{O}[\mathrm{C} @ @ \mathrm{H}] 1[\mathrm{C} @ @ \mathrm{H}]([\mathrm{C} @ @ \mathrm{H}]([\mathrm{C} @ \mathrm{H}]([\mathrm{C} @ @ \mathrm{H}](\mathrm{O} 1) \mathrm{C}) \mathrm{OCCNC}(=\mathrm{O}) / \mathrm{C}=\mathrm{C} / \mathrm{c} 1 \mathrm{c} \\
\mathrm{cccc} 1) \mathrm{O}) \mathrm{O}\end{array}$ \\
\hline 057_frag_301 & 1.8446 & 0.23 & 22 & -0.117 & 74 & O[C@@H]1CC[C@H]([C@@H](O1)C)OCCNC(=O)c1ccc(cc1)OC \\
\hline 058_frag_432 & 1.9219 & 0.18 & 23 & -0.117 & 127 & $\begin{array}{l}{[\mathrm{C} @ \mathrm{H}] 1([\mathrm{C} @ \mathrm{H}](\mathrm{CCCO} 1) \mathrm{O}[\mathrm{C} @ @ \mathrm{H}] 1[\mathrm{C} @ \mathrm{H}]([\mathrm{C} @ @ \mathrm{H}]([\mathrm{C} @ \mathrm{H}](\mathrm{CO} 1) \mathrm{O}) \mathrm{O}[\mathrm{C} @} \\
\mathrm{H}] 1 \mathrm{CCCCO}) \mathrm{O}) \mathrm{C}\end{array}$ \\
\hline 059_frag_192 & 2.1808 & 0.39 & 26 & -0.117 & 65 & $\mathrm{CC}(=\mathrm{O}) \mathrm{N}[\mathrm{C} @ @ \mathrm{H}](\mathrm{CCCNC}(\mathrm{N}) \mathrm{N}) \mathrm{C}(=\mathrm{O}) \mathrm{N}[\mathrm{C} @ @ \mathrm{H}](\mathrm{CCCNC}(\mathrm{N}) \mathrm{N}) \mathrm{C}(=\mathrm{O}) \mathrm{O}$ \\
\hline 060_frag_930 & 1.8719 & 0.56 & 22 & -0.119 & 52 & $\mathrm{CCCCCCNC}(=\mathrm{O})[\mathrm{C} @ @ \mathrm{H}](\mathrm{NC}(=\mathrm{O}) \mathrm{CCCCC}) \mathrm{CCCC}$ \\
\hline 061_frag_2844 & 1.9507 & 0.47 & 23 & -0.119 & 53 & $\mathrm{NCC}(=\mathrm{O}) \mathrm{N}[\mathrm{C} @ \mathrm{H}](\mathrm{C}(=\mathrm{O}) \mathrm{NCCCCNC}=\mathrm{N}) \mathrm{CCCNC}(\mathrm{N}) \mathrm{N}$ \\
\hline 062_frag_201 & 2.1371 & 0.39 & 25 & -0.12 & 131 & $\mathrm{C}(=\mathrm{O}) \mathrm{NC}(=\mathrm{O})[\mathrm{C} @ \mathrm{H}](\mathrm{CCCNC}(\mathrm{N}) \mathrm{N}) \mathrm{NC}(=\mathrm{O})[\mathrm{C} @ \mathrm{H}](\mathrm{CCCNC}(\mathrm{N}) \mathrm{N}) \mathrm{N}$ \\
\hline
\end{tabular}


Supporting Information (Texts S1-S9, Figures S1-S41 and Tables S1-S12)

\begin{tabular}{|c|c|c|c|c|c|c|}
\hline 063_frag_885 & 1.6359 & 0.35 & 19 & -0.121 & 49 & {$[\mathrm{C} @ \mathrm{H}] 1([\mathrm{C} @ @ \mathrm{H}]([\mathrm{C} @ \mathrm{H}]([\mathrm{C} @ @ \mathrm{H}]([\mathrm{C} @ \mathrm{H}](\mathrm{O} 1) \mathrm{CSCCCCCCC}) \mathrm{O}) \mathrm{N}) \mathrm{O}) \mathrm{O}$} \\
\hline 064_frag_218 & 1.9085 & 0.23 & 22 & -0.121 & 73 & $\mathrm{O}[\mathrm{C} @ @ \mathrm{H}] 1 \mathrm{C}[\mathrm{C} @ @ \mathrm{H}]([\mathrm{C} @ \mathrm{H}]([\mathrm{C} @ @ \mathrm{H}](\mathrm{O} 1) \mathrm{C}) \mathrm{OCCNC}(=\mathrm{O}) \mathrm{c} 1 \mathrm{ccccc} 1 \mathrm{I}) \mathrm{O}$ \\
\hline 065_frag_171 & 1.8439 & 0.18 & 21 & -0.123 & 60 & $\begin{array}{l}\mathrm{O}[\mathrm{C} @ \mathrm{H}] 1[\mathrm{C} @ @ \mathrm{H}](\mathrm{C}[\mathrm{C} @ @ \mathrm{H}](\mathrm{CO} 1) \mathrm{OS}(\mathrm{O})(\mathrm{O}) \mathrm{O}) \mathrm{O}[\mathrm{C} @ \mathrm{H}] 1[\mathrm{C} @ @ \mathrm{H}](\mathrm{CC}[\mathrm{C} @ \\
\mathrm{H}](\mathrm{O} 1) \mathrm{C}) \mathrm{O}\end{array}$ \\
\hline 066_frag_1108 & 1.9277 & 0.52 & 22 & -0.123 & 52 & NCCCCCCNC $(=\mathrm{O})[\mathrm{C} @ \mathrm{H}](\mathrm{CCCNC}(\mathrm{N}) \mathrm{N}) \mathrm{NC}(=\mathrm{O}) \mathrm{C}$ \\
\hline 067_frag_260 & 1.9361 & 0.21 & 22 & -0.123 & 74 & $\mathrm{O}[\mathrm{C} @ @ \mathrm{H}] 1 \mathrm{CC}[\mathrm{C} @ \mathrm{H}]([\mathrm{C} @ @ \mathrm{H}](\mathrm{O} 1) \mathrm{C}) \mathrm{OCCNC}(=\mathrm{O}) \mathrm{COc} 1 \mathrm{cccc} 1$ \\
\hline 068_frag_0 & 1.8545 & 0.3 & 21 & -0.124 & 56 & $\mathrm{C}(=\mathrm{O}) \mathrm{Nc} 1 \mathrm{cc}(\mathrm{cc}(\mathrm{c} 1 \mathrm{SCCN}) \mathrm{NC}(\mathrm{N}) \mathrm{N}) \mathrm{C}(\mathrm{C})(\mathrm{C}) \mathrm{C}$ \\
\hline 069_frag_1136 & 1.861 & 0.16 & 21 & -0.124 & 51 & CCCCO[C@H]1C[C@H]([C@H](CO[C@@H]2[C@H](CCCO2)OC)O1)O \\
\hline 070_frag_0 & 1.9112 & 0.3 & 21 & -0.127 & 55 & $\mathrm{C}(=\mathrm{O}) \mathrm{Nc} 1 \mathrm{cc}(\mathrm{cc}(\mathrm{c} 1 \mathrm{SCCNC}(\mathrm{N}) \mathrm{N}) \mathrm{N}) \mathrm{C}(\mathrm{C})(\mathrm{C}) \mathrm{C}$ \\
\hline 071_frag_1016 & 2.107 & 0.68 & 23 & -0.128 & 53 & $\mathrm{NCCCNC}(=\mathrm{O})[\mathrm{C} @ \mathrm{H}](\mathrm{CCCNC}(\mathrm{N}) \mathrm{N}) \mathrm{NC}(=\mathrm{O}) \mathrm{CCCCC}$ \\
\hline 072_frag_3739 & 2.1925 & 0.36 & 24 & -0.128 & 86 & $\mathrm{C}(\mathrm{C}(=\mathrm{O}) \mathrm{N}[\mathrm{C} @ @ \mathrm{H}](\mathrm{CCCC}) \mathrm{C}(=\mathrm{O}) \mathrm{N}[\mathrm{C} @ @ \mathrm{H}](\mathrm{C}(=\mathrm{O}) \mathrm{N}) \mathrm{CCCC}) \mathrm{CCCC}$ \\
\hline 073_frag_447 & 2.0279 & 0.17 & 22 & -0.129 & 126 & $\begin{array}{l}{[\mathrm{C} @ @ \mathrm{H}] 1(\mathrm{CCCCO} 1) \mathrm{O}[\mathrm{C} @ \mathrm{H}] 1 \mathrm{CO}[\mathrm{C} @ \mathrm{H}]([\mathrm{C} @ @ \mathrm{H}]([\mathrm{C} @ \mathrm{H}] 1 \mathrm{O}) \mathrm{O}) \mathrm{OC} 1 \mathrm{CCOC}} \\
\mathrm{C} 1\end{array}$ \\
\hline 074_frag_3 & 2.0621 & 0.21 & 22 & -0.131 & 97 & Cc1cec(cc1)N(Cc1eccec1)Cc1ccece 1 \\
\hline 075_frag_5946 & 1.602 & 0.28 & 17 & -0.132 & 47 & {$[\mathrm{C} @ @ \mathrm{H}] 1(\mathrm{CC}[\mathrm{C} @ \mathrm{H}]([\mathrm{C} @ @ \mathrm{H}](\mathrm{O} 1) \mathrm{OCCCCCCCC}) \mathrm{O}) \mathrm{C}$} \\
\hline 076_frag_1 & 2.2867 & 0.19 & 24 & -0.133 & 79 & $\mathrm{C}(=\mathrm{O})[\mathrm{C} @ \mathrm{H}](\mathrm{NC}(=\mathrm{O}) \mathrm{OCC} 1 \mathrm{c} 2 \mathrm{c}(\mathrm{c} 3 \mathrm{c} 1 \mathrm{cccc} 3) \mathrm{cccc} 2) \mathrm{CCN}$ \\
\hline 077_frag_518 & 1.8247 & 0.23 & 19 & -0.134 & 64 & $\begin{array}{l}\mathrm{C}(=\mathrm{O}) \mathrm{O}[\mathrm{C} @ \mathrm{H}] 1[\mathrm{C} @ @ \mathrm{H}]([\mathrm{C} @ \mathrm{H}]([\mathrm{C} @ \mathrm{H}](\mathrm{CO} 1) \mathrm{O}) \mathrm{O}) \mathrm{O}[\mathrm{C} @ @ \mathrm{H}] 1 \mathrm{CCC}[\mathrm{C} @ \mathrm{H}]( \\
\mathrm{O} 1) \mathrm{C}\end{array}$ \\
\hline 078_frag_130 & 2.1009 & 0.28 & 22 & -0.134 & 57 & $\mathrm{O}=\mathrm{C}(\mathrm{O}) \mathrm{CCc} 1 \mathrm{cn}(\mathrm{nn} 1) \mathrm{CC}(=\mathrm{O}) \mathrm{NCCCCNC}(\mathrm{N}) \mathrm{N}$ \\
\hline 079_frag_814 & 2.1062 & 0.36 & 22 & -0.134 & 52 & CCCCCCCC $[\mathrm{C} @ @ \mathrm{H}](\mathrm{CCCCCC}) \mathrm{OC}(=\mathrm{O}) \mathrm{CCCC}$ \\
\hline 080_frag_3 & 1.826 & 0.16 & 19 & -0.135 & 73 & $\mathrm{C}[\mathrm{C} @ @ \mathrm{H}] 1[\mathrm{C} @ \mathrm{H}]([\mathrm{C} @ @ \mathrm{H}]([\mathrm{C} @ @ \mathrm{H}]([\mathrm{C} @ @ \mathrm{H}](\mathrm{O} 1) \mathrm{O}) \mathrm{O}) \mathrm{NCc} 1 \mathrm{ccc}(\mathrm{cc} 1) \mathrm{F}) \mathrm{O}$ \\
\hline 081_frag_296 & 1.9329 & 0.34 & 20 & -0.135 & 50 & $\mathrm{C}(\mathrm{Cc} 1 \mathrm{ccc}(\mathrm{cc} 1) \mathrm{NC}(=\mathrm{O}) \mathrm{CCCN}) \mathrm{CCCCC}$ \\
\hline 082_frag_1442 & 1.937 & 0.2 & 20 & -0.136 & 122 & $\begin{array}{l}\mathrm{O}[\mathrm{C} @ \mathrm{H}] 1[\mathrm{C} @ @ \mathrm{H}]([\mathrm{C} @ @ \mathrm{H}]([\mathrm{C} @ \mathrm{H}]([\mathrm{C} @ @ \mathrm{H}](\mathrm{O} 1) \mathrm{C}) \mathrm{OC}(=\mathrm{O}) / \mathrm{C}(=\mathrm{C} / \mathrm{CCCC}) / \mathrm{C} \\
) \mathrm{O}) \mathrm{O}\end{array}$ \\
\hline 083_frag_57 & 1.4776 & 0.49 & 15 & -0.138 & 46 & $\mathrm{C}(=\mathrm{O}) \mathrm{c} 1 \mathrm{cc}[\mathrm{n}+](\mathrm{cc} 1) \mathrm{CCCCCCC}$ \\
\hline 084_frag_696 & 1.8842 & 0.31 & 19 & -0.139 & 49 & $\mathrm{C}(=\mathrm{O}) \mathrm{NCCCNC}(=\mathrm{O}) \mathrm{CNC}(=\mathrm{O}) \mathrm{CCCCCC}$ \\
\hline 085_frag_193 & 2.2825 & 0.31 & 23 & -0.139 & 54 & $\mathrm{C}(=\mathrm{O}) \mathrm{OCc} 1 \mathrm{cn}(\mathrm{nn} 1) \mathrm{CC}(=\mathrm{O}) \mathrm{N}[\mathrm{C} @ @ \mathrm{H}](\mathrm{CCCNC}(\mathrm{N}) \mathrm{N}) \mathrm{C}=\mathrm{O}$ \\
\hline 086_frag_639 & 1.816 & 0.27 & 18 & -0.141 & 48 & CCCCCCCC $[\mathrm{C} @ @ \mathrm{H}](\mathrm{CCCCCC}) \mathrm{OC}=\mathrm{O}$ \\
\hline 087_frag_107 & 1.628 & 0.62 & 16 & -0.142 & 46 & COCc1cc $[n+](\operatorname{cc} 1) C C C C C C C$ \\
\hline 088_frag_265 & 2.0281 & 0.21 & 20 & -0.142 & 71 & $\mathrm{O}[\mathrm{C} @ @ \mathrm{H}] 1 \mathrm{C}[\mathrm{C} @ @ \mathrm{H}]([\mathrm{C} @ \mathrm{H}]([\mathrm{C} @ @ \mathrm{H}](\mathrm{O} 1) \mathrm{C}) \mathrm{OCCNC}(=\mathrm{O}) / \mathrm{C}=\mathrm{C} / \mathrm{C}=\mathrm{C} / \mathrm{C}) \mathrm{O}$ \\
\hline 089_frag_242 & 1.9463 & 0.22 & 19 & -0.143 & 70 & $\mathrm{O}[\mathrm{C} @ @ \mathrm{H}] 1 \mathrm{C}[\mathrm{C} @ @ \mathrm{H}]([\mathrm{C} @ \mathrm{H}]([\mathrm{C} @ @ \mathrm{H}](\mathrm{O} 1) \mathrm{C}) \mathrm{OCCNC}(=\mathrm{O}) \mathrm{CCCCl}) \mathrm{O}$ \\
\hline 090_frag_901 & 1.8572 & 0.3 & 18 & -0.144 & 50 & C(C)CC[C@@H](C(C)C)O[C@H]1[C@@H]([C@@H]([C@@H](O1)CO)O)O \\
\hline 091_frag_300 & 1.4476 & 0.43 & 14 & -0.145 & 44 & $\operatorname{CCCCCCC}[n+] 1 \operatorname{cccc}(\mathrm{c} 1) \mathrm{C}$ \\
\hline 092_frag_329 & 1.7552 & 0.17 & 17 & -0.145 & 57 & {$[\mathrm{C} @ @ \mathrm{H}] 1(\mathrm{CCCO}[\mathrm{C} @ @ \mathrm{H}] 1 \mathrm{CO}) \mathrm{O}[\mathrm{C} @ @ \mathrm{H}] 1 \mathrm{O}[\mathrm{C} @ @ \mathrm{H}](\mathrm{CC}[\mathrm{C} @ \mathrm{H}] 1 \mathrm{O}) \mathrm{C}$} \\
\hline 093_frag_48 & 2.1546 & 0.45 & 20 & -0.151 & 79 & $\operatorname{COCCNC}(=\mathrm{O}) \mathrm{CCCCCCCCCCCCC}$ \\
\hline 094_frag_94 & 1.7607 & 0.2 & 16 & -0.154 & 58 & $\mathrm{C}(=\mathrm{O}) / \mathrm{C}=\mathrm{C} / \mathrm{CCCCCCCCCC}(\mathrm{C}) \mathrm{C}$ \\
\hline
\end{tabular}


Supporting Information (Texts S1-S9, Figures S1-S41 and Tables S1-S12)

\begin{tabular}{|c|c|c|c|c|c|c|}
\hline 095_frag_168 & 1.8744 & 0.23 & 17 & -0.154 & 68 & $\mathrm{O}[\mathrm{C} @ \mathrm{H}] 1 \mathrm{C}[\mathrm{C} @ @ \mathrm{H}]([\mathrm{C} @ \mathrm{H}]([\mathrm{C} @ @ \mathrm{H}](\mathrm{O} 1) \mathrm{C}) \mathrm{OC}(=\mathrm{O}) \mathrm{CCCCC}) \mathrm{O}$ \\
\hline 096_frag_196 & 2.0372 & 0.17 & 18 & -0.158 & 70 & $\mathrm{O}[\mathrm{C} @ @ \mathrm{H}] 1 \mathrm{CC}[\mathrm{C} @ \mathrm{H}]([\mathrm{C} @ @ \mathrm{H}](\mathrm{O} 1) \mathrm{C}) \mathrm{OCCNC}(=\mathrm{O}) \mathrm{C}(\mathrm{F})(\mathrm{F}) \mathrm{F}$ \\
\hline 097_frag_2578 & 1.7152 & 0.28 & 15 & -0.16 & 45 & $\mathrm{~N}(\mathrm{CCCCCCCC}) \mathrm{CCCNC}=\mathrm{O}$ \\
\hline 098_frag_77 & 2.0543 & 0.34 & 18 & -0.16 & 48 & $\operatorname{clccc}(\mathrm{C}(=\mathrm{O}) \mathrm{Nc} 2 \mathrm{cc}[\mathrm{n}+](\mathrm{cc} 2) \mathrm{CCC}) \mathrm{cc} 1$ \\
\hline 099_frag_338 & 1.9521 & 0.18 & 17 & -0.161 & 87 & $\begin{array}{l}{[\mathrm{C} @ \mathrm{H}] 1([\mathrm{C} @ @ \mathrm{H}]([\mathrm{C} @ \mathrm{H}]([\mathrm{C} @ \mathrm{H}](\mathrm{CO} 1) \mathrm{O}[\mathrm{C} @ \mathrm{H}] 1 \mathrm{CCC}[\mathrm{C} @ @ \mathrm{H}](\mathrm{O} 1) \mathrm{C}) \mathrm{O}) \mathrm{O})} \\
\mathrm{C}\end{array}$ \\
\hline 100_frag_1 & 1.7376 & 0.2 & 15 & -0.162 & 58 & $\mathrm{OC}[\mathrm{C} @ @ \mathrm{H}](\mathrm{C}[\mathrm{N}+](\mathrm{CCCC})(\mathrm{C}) \mathrm{CCCC}) \mathrm{O}$ \\
\hline 101_frag_4 & 2.2111 & 0.17 & 19 & -0.163 & 95 & c1ccc(cc1)NC(=O)[C@]12C[C@H]3C[C@@ \\
\hline 102_frag_1657 & 2.2821 & 0.17 & 19 & -0.168 & 58 & $\mathrm{C} 1[\mathrm{C} @ @ \mathrm{H}]([\mathrm{C} @ \mathrm{H}](\mathrm{C}[\mathrm{C} @ \mathrm{H}](\mathrm{O} 1) \mathrm{C}(=\mathrm{O}) \mathrm{OC}) \mathrm{O}[\mathrm{C} @ \mathrm{H}] 1 \mathrm{CCC}[\mathrm{C} @ \mathrm{H}](\mathrm{O} 1) \mathrm{C}) \mathrm{O}$ \\
\hline 103_frag_66 & 1.9906 & 0.18 & 16 & -0.174 & 46 & $\mathrm{C}(=\mathrm{O}) \mathrm{NCCCNCCCNCCCCN}$ \\
\hline 104_frag_192 & 1.8 & 0.3 & 14 & -0.18 & 44 & $\mathrm{CCCC}[\mathrm{n}+] 1 \mathrm{ccc}(\mathrm{c}(\mathrm{c} 1) \mathrm{C}) \mathrm{C}(\mathrm{C}) \mathrm{C}$ \\
\hline 105_frag_3 & 2.272 & 0.23 & 17 & -0.187 & 92 & $\mathrm{Cc} 1 \mathrm{ccc}(\operatorname{cc} 1) \mathrm{NC}(=\mathrm{O}) \mathrm{Cc} 1 \mathrm{ccccc} 1$ \\
\hline 106_frag_62 & 1.6151 & 0.34 & 12 & -0.188 & 73 & $\mathrm{OC}(=\mathrm{O}) \mathrm{CCCCCCCCC}$ \\
\hline 107_frag_21 & 2.0272 & 0.16 & 15 & -0.189 & 49 & $\mathrm{C}(=\mathrm{O}) \mathrm{NCCCN} 1 \mathrm{CCN}(\mathrm{CC} 1) \mathrm{CCC}$ \\
\hline 108_frag_820 & 1.7755 & 0.16 & 13 & -0.191 & 43 & CCCCO[C@H]1CC[C@H]([C@H](O1)C)O \\
\hline 109_frag_293 & 2.0421 & 0.16 & 15 & -0.191 & 60 & $\mathrm{C} 1 \mathrm{C}[\mathrm{C} @ \mathrm{H}]([\mathrm{C} @ \mathrm{H}](\mathrm{O}[\mathrm{C} @ @ \mathrm{H}] 1 \mathrm{C}) \mathrm{O}) \mathrm{OC}(=\mathrm{O}) \mathrm{CCC}=\mathrm{O}$ \\
\hline 110_frag_418 & 1.9428 & 0.23 & 14 & -0.194 & 44 & $\operatorname{CCCCCN}(\mathrm{CC}(=\mathrm{O}) \mathrm{O}) \mathrm{CC}(=\mathrm{O}) \mathrm{O}$ \\
\hline 111_frag_47 & 1.7071 & 0.42 & 12 & -0.199 & 42 & $\mathrm{Nc} 1 \mathrm{cc}[\mathrm{n}+](\mathrm{cc} 1) \mathrm{CCCCC}$ \\
\hline 112_frag_2146 & 1.8455 & 0.19 & 13 & -0.199 & 43 & {$[\mathrm{~N}+](\mathrm{C})(\mathrm{C})(\mathrm{CC}) \mathrm{CCC}[\mathrm{N}+](\mathrm{C})(\mathrm{C}) \mathrm{CC}$} \\
\hline 113_frag_78 & 1.7346 & 0.32 & 12 & -0.202 & 42 & $\mathrm{C} 1 \mathrm{CN}(\mathrm{CN} 1 \mathrm{CCCCCC}) \mathrm{C}$ \\
\hline 114_frag_253 & 2.0471 & 0.22 & 14 & -0.205 & 70 & $\mathrm{C}(=\mathrm{O}) \mathrm{N}[\mathrm{C} @ @ \mathrm{H}](\mathrm{Cc} 1 \operatorname{ccccc} 1) \mathrm{C}(=\mathrm{O}) \mathrm{O}$ \\
\hline 115_frag_258 & 1.9091 & 0.17 & 13 & -0.206 & 43 & $\mathrm{CC}(\mathrm{C}) \mathrm{O}[\mathrm{C} @ \mathrm{H}] 1[\mathrm{C} @ @ \mathrm{H}](\mathrm{CC}[\mathrm{C} @ \mathrm{H}](\mathrm{O} 1) \mathrm{CO}) \mathrm{O}$ \\
\hline 116_frag_110 & 2.0899 & 0.33 & 14 & -0.209 & 44 & $\mathrm{CCCCCNC}(=\mathrm{O}) \mathrm{c} 1 \mathrm{ccncc} 1$ \\
\hline 117_frag_556 & 2.1482 & 0.16 & 14 & -0.215 & 46 & $\operatorname{clc}(\operatorname{cc}(\operatorname{cc} 10 C C N) \mathrm{C}=\mathrm{O}) \mathrm{C}=\mathrm{O}$ \\
\hline 118_frag_33 & 1.8817 & 0.34 & 12 & -0.22 & 42 & $\mathrm{c} 1[\mathrm{n}+](\mathrm{cc}(\mathrm{cc} 1) \mathrm{CO}) \mathrm{CCCC}$ \\
\hline 119_frag_1 & 2.0421 & 0.19 & 13 & -0.22 & 54 & OCCCCCCCN(CC)CC \\
\hline 120_frag_23 & 1.8992 & 0.17 & 12 & -0.222 & 42 & $\mathrm{c} 1 \mathrm{c}(\mathrm{c}(\mathrm{cc}(\mathrm{c} 1) \mathrm{CC}) \mathrm{N}) \mathrm{SCC}$ \\
\hline 121_frag_113 & 2.2369 & 0.17 & 14 & -0.224 & 66 & {$[\mathrm{C} @ \mathrm{H}] 1(\mathrm{OCC}[\mathrm{C} @ @ \mathrm{H}] 1 \mathrm{OC}(=\mathrm{O}) \mathrm{C}) \mathrm{COC}(=\mathrm{O}) \mathrm{C}$} \\
\hline $122 \_$frag_ 8 & 2.1085 & 0.26 & 13 & -0.227 & 98 & $\mathrm{C}(\mathrm{c} 1 \mathrm{ccccc} 1) \mathrm{c} 1 \mathrm{ccccc} 1$ \\
\hline 123_frag_27 & 2.0151 & 0.32 & 12 & -0.235 & 42 & $\mathrm{CSCc} 1 \mathrm{cc}[\mathrm{n}+](\mathrm{cc} 1) \mathrm{CCC}$ \\
\hline 124_frag_39 & 2.0252 & 0.28 & 12 & -0.236 & 42 & $\mathrm{CSSCCc} 1 \mathrm{cc}[\mathrm{n}+](\mathrm{cc} 1) \mathrm{C}$ \\
\hline 125_frag_72 & 2.0505 & 0.17 & 12 & -0.239 & 42 & {$[\mathrm{C} @ @ \mathrm{H}] 1(\mathrm{CCCO}[\mathrm{C} @ @ \mathrm{H}] 1 \mathrm{C}) \mathrm{OS}(\mathrm{O})(\mathrm{O}) \mathrm{O}$} \\
\hline 126_frag_254 & 2.067 & 0.3 & 12 & -0.241 & 69 & $\mathrm{C}(=\mathrm{O}) \mathrm{NCc} 1 \mathrm{cc}(\mathrm{c}(\mathrm{cc} 1) \mathrm{O}) \mathrm{O}$ \\
\hline 127_frag_1864 & 2.0919 & 0.16 & 12 & -0.244 & 42 & {$[\mathrm{~N}+](\mathrm{C})(\mathrm{C})(\mathrm{CC}) \mathrm{CC}[\mathrm{N}+](\mathrm{C})(\mathrm{C}) \mathrm{CC}$} \\
\hline 128_frag_450 & 2.0981 & 0.2 & 12 & -0.245 & 84 & $\mathrm{C}(\mathrm{CC}[\mathrm{C} @ @](\mathrm{C}(\mathrm{C}) \mathrm{C})(\mathrm{C}) \mathrm{OC}=\mathrm{O}) \mathrm{C}$ \\
\hline 129_frag_48 & 2.274 & 0.24 & 13 & -0.245 & 72 & $\operatorname{COCCNC}(=\mathrm{O}) \mathrm{c} 1 \mathrm{ccccc} 1$ \\
\hline
\end{tabular}


Supporting Information (Texts S1-S9, Figures S1-S41 and Tables S1-S12)

\begin{tabular}{|c|c|c|c|c|c|c|}
\hline 130_frag_318 & 2.1104 & 0.19 & 12 & -0.246 & 63 & $\mathrm{C}(=\mathrm{O}) \mathrm{OCCCCCCON}=\mathrm{O}$ \\
\hline 131_frag_11 & 1.8531 & 0.26 & 10 & -0.259 & 40 & $\mathrm{c} 1[\mathrm{n}+](\mathrm{cccc} 1) \mathrm{CCCC}$ \\
\hline 132_frag_242 & 2.0624 & 0.16 & 11 & -0.262 & 70 & $\mathrm{C}(\mathrm{CC}[\mathrm{C} @ @](\mathrm{C}(\mathrm{C})(\mathrm{C}) \mathrm{C})(\mathrm{C}) \mathrm{O}) \mathrm{C}$ \\
\hline 133_frag_139 & 2.2967 & 0.17 & 12 & -0.268 & 110 & $\mathrm{C} 1 \mathrm{CC}[\mathrm{C} @ \mathrm{H}]([\mathrm{C} @ @ \mathrm{H}](\mathrm{O} 1) \mathrm{COC}(=\mathrm{O}) \mathrm{C}) \mathrm{O}$ \\
\hline 134_frag_82 & 1.9884 & 0.17 & 10 & -0.278 & 43 & $\mathrm{C}(\mathrm{C}) \mathrm{CC}[\mathrm{C} @ @ \mathrm{H}](\mathrm{C}(\mathrm{C}) \mathrm{C}) \mathrm{CC}$ \\
\hline 135_frag_260 & 2.0589 & 0.23 & 10 & -0.288 & 67 & $\mathrm{C}(=\mathrm{O}) \mathrm{NCc} 1 \mathrm{ccccc} 1$ \\
\hline 136_frag_1 & 2.0778 & 0.19 & 10 & -0.291 & 48 & $\mathrm{OCCCCN}(\mathrm{CC}) \mathrm{CC}$ \\
\hline 137_frag_42 & 1.9692 & 0.18 & 9 & -0.306 & 39 & NCCCNCCCC \\
\hline 138_frag_1 & 1.7831 & 0.2 & 8 & -0.312 & 77 & $\mathrm{C}(\mathrm{CCC}=\mathrm{C}(\mathrm{C}) \mathrm{C}) \mathrm{C}$ \\
\hline 139_frag_317 & 2.2486 & 0.16 & 10 & -0.315 & 60 & $\mathrm{C}(=\mathrm{O}) \mathrm{NCCN}(\mathrm{CC}) \mathrm{CC}$ \\
\hline 140_frag_78 & 2.2511 & 0.19 & 10 & -0.315 & 40 & $\mathrm{CC}[\mathrm{n}+] 1 \mathrm{ccc}(\mathrm{cc} 1) \mathrm{NC}$ \\
\hline 141_frag_1 & 2.0999 & 0.16 & 9 & -0.327 & 46 & OCCCN(CC)CC \\
\hline 142_frag_986 & 2.1526 & 0.17 & 9 & -0.335 & 83 & $\mathrm{C}(\mathrm{O}) \mathrm{CC}[\mathrm{C} @ \mathrm{H}](\mathrm{C}(=\mathrm{C}) \mathrm{C}) \mathrm{O}$ \\
\hline 143_frag_581 & 2.2404 & 0.17 & 9 & -0.349 & 57 & {$[\mathrm{C} @ @ \mathrm{H}](\mathrm{O})(\mathrm{CCCC}(=\mathrm{C}) \mathrm{C}) \mathrm{C}$} \\
\hline 144_frag_8926 & 2.3006 & 0.18 & 9 & -0.358 & 39 & {$[\mathrm{~N}+](\mathrm{C})(\mathrm{C})(\mathrm{CC}=\mathrm{O}) \mathrm{CCC}$} \\
\hline 145_frag_115 & 2.2209 & 0.16 & 8 & -0.389 & 39 & $\mathrm{OC}(=\mathrm{O})[\mathrm{C} @ \mathrm{H}](\mathrm{CCC}) \mathrm{N}$ \\
\hline 146_frag_44 & 2.2476 & 0.17 & 8 & -0.393 & 54 & CCCN[C@@ @ $]$ ](C)CO \\
\hline 147_frag_340 & 2.2752 & 0.17 & 8 & -0.398 & 40 & $\mathrm{O}(\mathrm{CCCCOC}) \mathrm{C}$ \\
\hline 148_frag_982 & 2.2841 & 0.16 & 8 & -0.4 & 83 & $\mathrm{C}(\mathrm{C} / \mathrm{C}=\mathrm{C} / \mathrm{C}(\mathrm{C}) \mathrm{C}) \mathrm{C}$ \\
\hline 149_frag_72 & 2.1633 & 0.2 & 7 & -0.433 & 52 & $\mathrm{NCCNC}(\mathrm{N}) \mathrm{N}$ \\
\hline 150_frag_9 & 2.1834 & 0.16 & 7 & -0.437 & 46 & CCCNCCN \\
\hline 151_frag_0 & 2.2518 & 0.24 & 7 & -0.45 & 88 & $\mathrm{CCCNC}(\mathrm{N}) \mathrm{N}$ \\
\hline 152_frag_0 & 2.1411 & 0.16 & 6 & -0.5 & 77 & $\mathrm{CCCC}(\mathrm{C}) \mathrm{C}$ \\
\hline 153_frag_20 & 2.2594 & 0.19 & 6 & -0.527 & 101 & $\mathrm{C}(=\mathrm{O}) \mathrm{NCCC}$ \\
\hline 154_frag_0 & 2.2998 & 0.18 & 6 & -0.537 & 77 & $\mathrm{CCC}=\mathrm{C}(\mathrm{C}) \mathrm{C}$ \\
\hline 155_frag_0 & 1.9634 & 0.21 & 5 & -0.55 & 103 & $\mathrm{CCCCN}$ \\
\hline
\end{tabular}

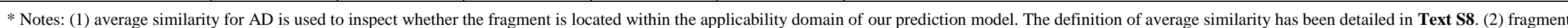
size refers to the total number of heavy atoms in the fragment; (3) parent molecule size refers to the number of heavy atom in the original molecule that is used to generate this fragment by recursive fragmentation method. 
Supporting Information (Texts S1-S9, Figures S1-S41 and Tables S1-S12)

Table S12. 10 fragments from recursive of Dataset-External-Test (70 compounds).

\begin{tabular}{|c|c|c|c|c|c|c|}
\hline Fragment name & $\begin{array}{c}\text { Predicted } \\
\text { LogHD }_{50}\end{array}$ & $\begin{array}{c}\text { Average } \\
\text { similarity } \\
\text { for AD* }\end{array}$ & $\begin{array}{c}\text { Fragment } \\
\text { size* }^{*}\end{array}$ & $\begin{array}{l}\text { Fragment } \\
\text { efficiency }\end{array}$ & $\begin{array}{c}\text { Parent } \\
\text { molecule } \\
\text { size* }\end{array}$ & SMILE string \\
\hline 001_frag_3544 & 1.6436 & 0.34 & 26 & -0.089 & 103 & $\mathrm{~N}([\mathrm{C} @ \mathrm{H}](\mathrm{C}(=\mathrm{O}) \mathrm{N}[\mathrm{C} @ \mathrm{H}](\mathrm{C}(=\mathrm{O}) \mathrm{NCCCCC}) \mathrm{CCC}) \mathrm{C}=\mathrm{O}) \mathrm{C}(=\mathrm{O}) \mathrm{CCC}(\mathrm{C}) \mathrm{C}$ \\
\hline 002_frag_1572 & 1.7949 & 0.16 & 24 & -0.105 & 103 & $\begin{array}{l}{[\mathrm{C} @ \mathrm{H}] 1(\mathrm{CCCO}[\mathrm{C} @ \mathrm{H}] 1 \mathrm{C}) \mathrm{O}[\mathrm{C} @ \mathrm{H}] 1[\mathrm{C} @ @ \mathrm{H}]([\mathrm{C} @ \mathrm{H}](\mathrm{CCO} 1) \mathrm{O}[\mathrm{C} @ \mathrm{H}] 1 \mathrm{CCC}[} \\
\mathrm{C} @ \mathrm{H}](\mathrm{O} 1) \mathrm{CO}) \mathrm{O}\end{array}$ \\
\hline 003_frag_2074 & 1.8687 & 0.16 & 25 & -0.105 & 103 & $\begin{array}{l}{[\mathrm{C} @ \mathrm{H}] 1(\mathrm{CCCOC} 1) \mathrm{O}[\mathrm{C} @ \mathrm{H}] 1 \mathrm{C}[\mathrm{C} @ \mathrm{H}](\mathrm{CCO} 1) \mathrm{O}[\mathrm{C} @ \mathrm{H}] 1 \mathrm{C}[\mathrm{C} @ @ \mathrm{H}]([\mathrm{C} @ \mathrm{H}]([\mathrm{C}} \\
@ \mathrm{H}](\mathrm{O} 1) \mathrm{CO}) \mathrm{O}) \mathrm{OC}\end{array}$ \\
\hline 004_frag_24 & 1.7419 & 0.17 & 23 & -0.106 & 86 & $\begin{array}{l}\mathrm{C} 1[\mathrm{C} @ @ \mathrm{H}](\mathrm{C}([\mathrm{C} @ @ \mathrm{H}] 2[\mathrm{C} @](\mathrm{C} 1)(\mathrm{C} 1=\mathrm{CC}[\mathrm{C} @] 3([\mathrm{C} @]([\mathrm{C} @ @ \mathrm{H}] 1 \mathrm{CC} 2)(\mathrm{CC}= \\
\mathrm{C} 3) \mathrm{C}) \mathrm{C}) \mathrm{C})(\mathrm{C}) \mathrm{C}) \mathrm{O}\end{array}$ \\
\hline 005_frag_613 & 1.7955 & 0.24 & 19 & -0.132 & 49 & $\operatorname{NCCN}(\mathrm{C}(=\mathrm{O}) \mathrm{CCc} 1 \operatorname{ccccc} 1)[\mathrm{C} @ \mathrm{H}](\mathrm{C}=\mathrm{O}) \mathrm{CC}$ \\
\hline 006_frag_1660 & 1.8677 & 0.16 & 19 & -0.138 & 106 & $\begin{array}{l}\mathrm{C} 1[\mathrm{C} @ @ \mathrm{H}](\mathrm{CCO}[\mathrm{C} @ @ \mathrm{H}] 1 \mathrm{C}) \mathrm{O}[\mathrm{C} @ \mathrm{H}] 1[\mathrm{C} @ @ \mathrm{H}]([\mathrm{C} @ @ \mathrm{H}](\mathrm{C}[\mathrm{C} @ \mathrm{H}](\mathrm{O} 1) \mathrm{CO} \\
) \mathrm{OC}) \mathrm{O}\end{array}$ \\
\hline 007_frag_94 & 1.8326 & 0.24 & 17 & -0.151 & 47 & $\mathrm{C}([\mathrm{C} @ @ \mathrm{H}](\mathrm{Cc} 1 \mathrm{ccccc} 1) \mathrm{C}=\mathrm{O}) \mathrm{C}(=\mathrm{O}) \mathrm{CCCN}$ \\
\hline 008_frag_62 & 1.3392 & 0.47 & 11 & -0.17 & 41 & $\mathrm{C}[\mathrm{N}+](\mathrm{C})(\mathrm{C}) \mathrm{CCCCCCC}$ \\
\hline 009_frag_39 & 1.7377 & 0.29 & 10 & -0.243 & 41 & $\mathrm{~N}(\mathrm{C}=\mathrm{O}) \mathrm{CCCCCCC}$ \\
\hline 010_frag_1133 & 2.1103 & 0.18 & 9 & -0.328 & 101 & $\mathrm{C}(\mathrm{CCC}(\mathrm{C}) \mathrm{C}) \mathrm{OC}=\mathrm{O}$ \\
\hline
\end{tabular}

* Notes: (1) the average similarity for AD is used to inspect whether the fragment is located within the applicability domain of our prediction model. The definition of average similarity has been detailed in Text S8. (2)

fragment size refers to the total number of heavy atoms in the fragment; (3) parent molecule size refers to the number of heavy atom in the original molecule that is used to generate this fragment by recursive fragmentation method. 
This is the end of supporting information. 